\title{
Floods of September-October 1967 in South Texas and Northeastern Mexico
}

GEOLOGICAL SURVEY WATER-SUPPLY PAPER 1880-B

Prepared in cooperation with the Texas Water Development Board

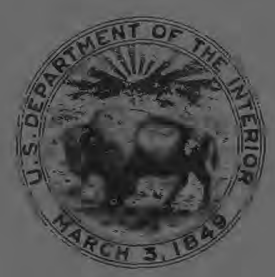




\section{Floods of September-October 1967 in South Texas and Northeastern Mexico}

By ELMER E. SCHROEDER, R. U. GROZIER, D. C. HAHL, and A. E. HULME

FLOODS OF 1967 IN THE UNITED STATES

GEOLOGICAL SURVEY WATER-SUPPLY PAPER 1880-B

Prepared in cooperation with the Texas Water Development Board

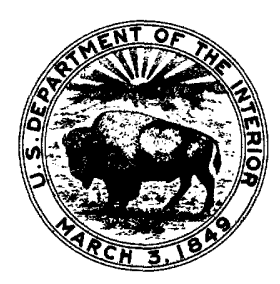




\section{UNITED STATES DEPARTMENT OF THE INTERIOR}

ROGERS C. B. MORTON, Secretary

\section{GEOLOGICAL SURVEY}

V. E. McKelvey, Director

\footnotetext{
Library of Congress Cataloging in Publication Data

Floods of September-October 1967 in south Texas and northeastern Mexico.

(Floods of 1967 in the United States) (Geological Survey Water-Supply Paper 1880-B)

"Prepared in cooperation with the Texas Water Development Board."

Bibliography: p.

Supt. of Docs. No.: I 19.13:1880-B

1. Floods-Texas. 2. Floods-Mexico. I. Schroeder, Elmer E. II. Texas. Water Development Board. III. Series. IV. Series: United States. Geological Survey. Water-Supply Paper 1880-B.

TC801.U2 no. 1880-B [GB1225.T4] 553'.7'0973s [551.4'8] 74-16470
}

For sale by the Superintendent of Documents, U. S. Government Printing Office Washington, D. C. 20402 - Price $\$ 3.55 \quad$ (paper cover)

Stock Number 2401-02562 


\section{CONTENTS}

Definitions of terms and abbreviations $\ldots \ldots \ldots \ldots \ldots \ldots \ldots \ldots \ldots \ldots \ldots$, VI

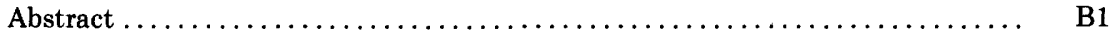

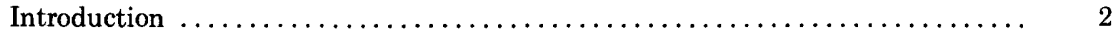

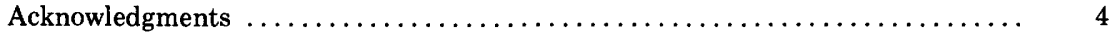

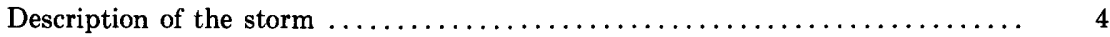

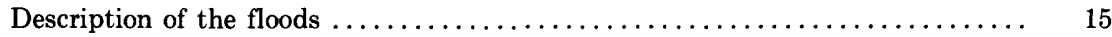

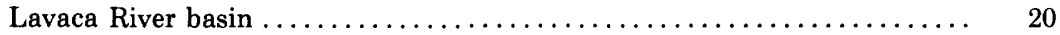

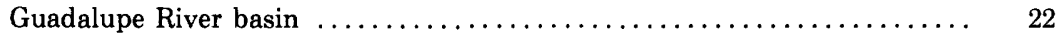

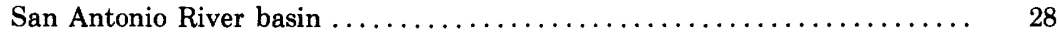

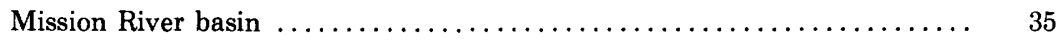

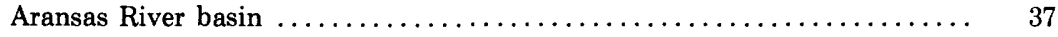

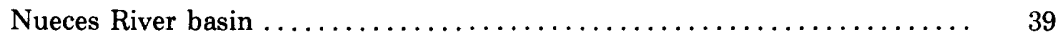

Coastal area between the Nueces River and the Rio Grande .......... 44

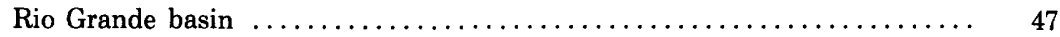

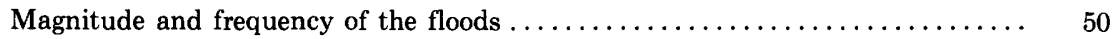

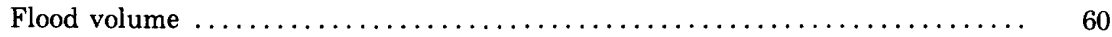

Summary of flood damage $\ldots \ldots \ldots \ldots \ldots \ldots \ldots \ldots \ldots \ldots \ldots \ldots \ldots \ldots \ldots \ldots \ldots$

Results of freshwater inflow to bays and estuaries, by D. C. Hahl ......... 67

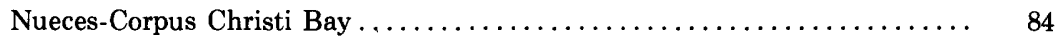

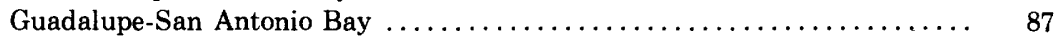

Effects of the hurricane on water levels in wells, by Texas Water Development

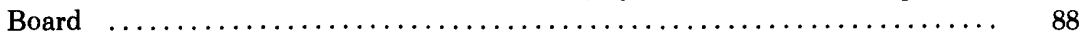

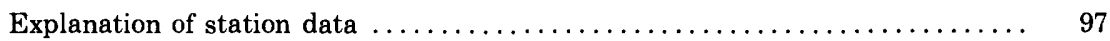

Summary of flood stages and discharges $\ldots \ldots \ldots \ldots \ldots \ldots \ldots \ldots \ldots \ldots, 108$

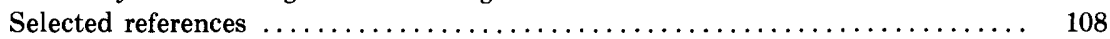

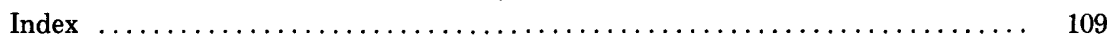

\section{ILLUSTRATIONS}

Plate 1. Maps showing data on Hurricane Beulah, September-October 1967, south Texas and northeastern Mexico ........... In pocket

2. Map showing flooding by Poesta Creek at Beeville ......... In pocket

3. Graph showing concentrations of dissolved solids, chloride, and dissolved oxygen in the Nueces River and Corpus Christi Bay and in the Guadalupe and San Antonio Rivers and San

Antonio Bay ................................ In pocket

FIGURE 1. Map showing track of Hurricane Beulah $\ldots \ldots \ldots \ldots \ldots \ldots \ldots$

2. Graph showing accumulation of rainfall at Falfurrias, Whitsett,

Mission, and Three Rivers, September 19-25, 1967 .........

3. Graph showing accumulation of rainfall at Cibolo Creek, Goliad,

Sinton, and Beeville, September 19-25, $1967 \ldots \ldots \ldots \ldots \ldots$. 
Figure 4. Graph showing accumulation of rainfall at Sombreretillo, Nuevo Leon; Difunto Angel, Tamaulipas; Ciudad Mier, Tamaulipas; Bajo Rio San Juan, Tamaulipas; and Villa de Santiago, Nuevo Leon, Mexico, September 19-25, $1967 \ldots \ldots \ldots \ldots \ldots \ldots \ldots$

5. Isohyetal and location of rain-gage network on the King Ranch . B8

6. Map showing location of U.S. Weather Bureau recording rain gages 22 7-20. Graphs showing:

7. Rainfall intensity-duration-frequency curve for Corpus Christi and the maximum point rainfall intensity at U.S. Weather Bureau rain gage 0639, Beeville 5 NE., September $21,1967 \ldots \ldots \ldots \ldots \ldots \ldots \ldots \ldots \ldots \ldots$.

8. Rainfall intensity-duration-frequency curve for San Antonio and the maximum point rainfall intensity at U.S. Weather Bureau rain gage 0639, Beeville $5 \mathrm{NE}$, September 21, $1967 \ldots \ldots \ldots \ldots \ldots \ldots \ldots \ldots \ldots$

9. Barometric pressure and wind velocity at the U.S. Weather Bureau station, Corpus Christi, Tex ........

10. Barometric pressure and wind velocity at the U.S. Weather Bureau station, Victoria, Tex .............

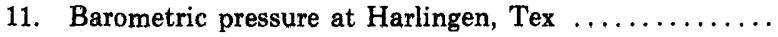

12. Barometric pressure and wind velocity at McAllen, Tex.,

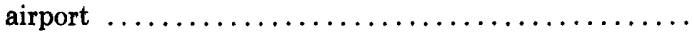

13. Barometric pressure and wind velocity at Brownsville,

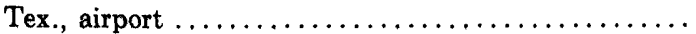

14. Barometric pressure at Port Isabel Coast Guard Station, South Padre Island $\ldots \ldots \ldots \ldots \ldots \ldots \ldots \ldots \ldots$

15. Tide elevation at Matagorda Peninsula gage ..........

16. Tide elevation in Lavaca Bay at Interstate Highway 35 causeway ...............................

17. Tide elevation in San Antonio Bay at Benderwald Point

18. Tide elevation in Copano Bay at Bayside ............

19. Tide elevation in Corpus Christi Bay at the tidal basin

20. Tide elevation in Corpus Christi Bay at the Naval Air Force Station ...........................

21. Graph showing accumulated weighted rainfall and net runoff, Escondido Creek subwatershed $1 \ldots \ldots \ldots \ldots \ldots \ldots \ldots$.

22. Graph showing accumulated weighted rainfall and net runoff, Escondido Creek subwatershed $11 \ldots \ldots \ldots \ldots \ldots \ldots \ldots$

23. Graph showing accumulated weighted rainfall and net runoff, Escondido Creek at Kenedy .........................

24. Discharge hydrographs for Guadalupe River at Victoria, Coleto Creek near Schroeder, and San Antonio River at Goliad ......

25. Flood profile of Poesta Creek at Beeville, September 21, 1967 ..

26. Discharge hydrograph for Nueces River at Simmons, Frio River near Calliham, Atascosa River at Whitsett, and Nueces River at

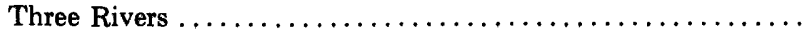

27. Discharge hydrograph for Nueces River near Three Rivers and Nueces River near Mathis ........................

28. Profile of water-surface elevations along St. Marys Street (U.S. Highway 281) in Falfurrias, September 23, 1967 ...........

29. Discharge hydrographs for the United States floadways and the main-stem Rio Grande below Anzalduas Dam ..............

30. Discharge hydrographs for each main-stem Rio Grande gaging station downstream from Falcon Dam $\ldots \ldots \ldots \ldots \ldots \ldots \ldots \ldots$. 
FIGURE $\quad 31$. Flood-distribution graph for San Antonio River at Goliad ......

32. Flood-distribution graph for Mission River at Refugio ..........

33. Flood-distribution graph for Nueces River at Three Rivers .....

34, 35. Regional flood-frequency curves for selected stations $\ldots \ldots \ldots 55,56$

36. Graph showing peak discharge on the main-stem Nueces River below West Nueces River and the relation of discharge to distance above the mouth for selected recurrence intervals ...........

37. Graph showing peak discharge on the Frio River below Dry Frio River and the relation of discharge to distance above the mouth

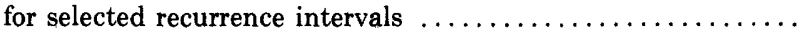

38. Graph showing relation of unit peak discharge and drainage area

39-41. Pictographs of runoff volume for selected drainage areas .....60, 61

42. Graph showing relation of drainage area and runoff, in inches, during the Hurricane Beulah storm period .................

43. Graph showing relation of drainage area and runoff, in acre-feet, during the Beulah storm period $\ldots \ldots \ldots \ldots \ldots \ldots \ldots \ldots \ldots$

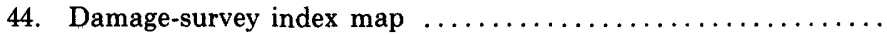

45. Graph showing periods of data collection and stages of Corpus Christi and San Antonio Bays, September 28-October 3, 1967.

46. Graph showing the relationship of dissolved solids and chloride concentrations to specific conductance in Corpus Christi and San

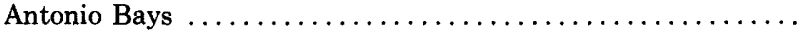

47-50. Maps showing location of:

47. Quality-of-water data-collection sites in Nueces-Corpus

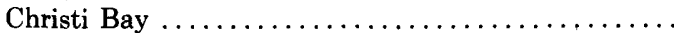

48. Quality-of-water data-collection sites in San Antonio Bay

49. Wells and changes in water levels in the Alice-Corpus Christi area

50. Wells and changes in water levels in the lower Rio Grande Valley 5

51. Hydrographs of wells and precipitation at nearby stations in the Alice-Corpus Christi area ..........................

52. Hydrographs of wells and precipitation at nearby stations in the lower Rio Grande Valley ..........................

\section{TABLES}

TABlE 1. Precipitation at U.S. Weather Bureau gages, storm period September 19-25, $1967 \ldots \ldots \ldots \ldots \ldots \ldots \ldots \ldots \ldots \ldots \ldots$

2. Precipitation at supplemental sites, storm period September 19-25,

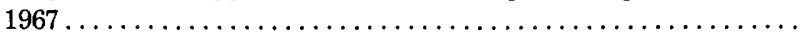

3. Precipitation at weather stations in Mexico, storm period

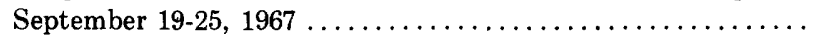

4. Precipitation at King Ranch rain gages, storm period September

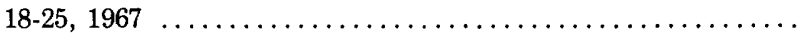

5. Hourly precipitation at U.S. Weather Bureau weighing rain gages,

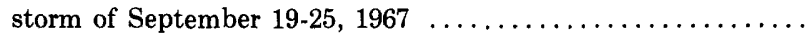

6. Summary of hurricane damages by areas in south central Texas

7. Summary of damages at selected Texas cities affected by

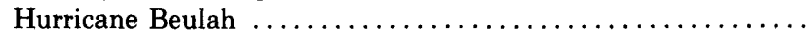

8. Suspended-sediment concentrations in the Nueces River and Cor-

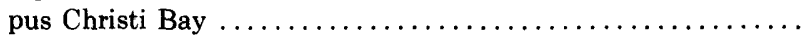


T $\mathrm{T}$ ABLE 9. Chemical analyses of water in the Nueces River and in Corpus Christi Bay, September 28-30, 1967 ................ B69

10. Field determinations of water-quality data for Corpus Christi Bay,

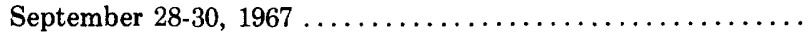

11. Chemical analyses of water in the Nueces River and in Corpus Christi Bay, October 2-3, $1967 \ldots \ldots \ldots \ldots \ldots \ldots \ldots \ldots \ldots$

12. Field determinations of water-quality data for Corpus Christi Bay,

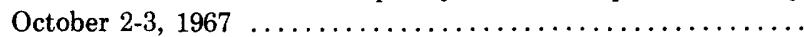

13. Chemical analyses of water in the Guadalupe and San Antonio Rivers and in San Antonio Bay, September 27 and October 1, 1967

14. Field determinations of water-quality data for San Antonio Bay,

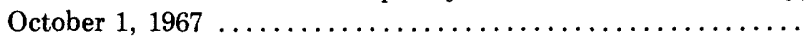

15. Water levels in wells in the Alice-Corpus Christi area .........

16. Water levels in wells in the lower Rio Grande Valley .............

17. Summary of flood stages and discharges ............... 100

\section{DEFINITION OF TERMS AND ABBREVIATIONS}

The terms and abbreviations of streamflow and other hydrologic data, as used in this report, are defined as follows:

Gaging station is a particular site on a stream, canal, lake, or reservoir where systematic observations of gage height or discharge are obtained. When used in connection with a discharge record, the term is applied only to gaging stations where a continuous record of discharge is obtained.

Crest-stage station is a particular site where limited streamflow data on peak stages are collected systematically over a period of years for use in hydrologic analyses.

Cubic foot per second (cfs) is the rate of discharge of a stream whose channel is 1 square foot in cross-sectional area and whose average velocity is 1 foot per second. The volume of water represented by a flow of 1 cubic foot per second for 24 hours is equivalent to 86,400 cubic feet, 1.983471 acre-feet, or 646,317 gallons.

Cubic feet per second per square mile (cfs per sq $\mathrm{mi}$ ) is the average number of cubic feet of water flowing per second from each square mile of area drained, assuming that the runoff is distributed uniformly in time and area.

Acre-foot (acre-ft) is the quantity of water required to cover an acre to a depth of 1 foot and is equivalent to 43,560 cubic feet, or 325,851 gallons. The term is generally used in relation to storage and volume of runoff.

Runoff, in inches (in.) is the depth to which the drainage area would be covered if all the runoff for a given time period were uniformly distributed on its surface.

Drainage area of a stream at a specified location is that area, measured in a horizontal plane, which is so enclosed by a topographic divide that direct surface runoff from precipitation normally would drain by gravity into the stream above the specified point. Drainage area is expressed in square miles in this report.

Contents is the volume of water in a reservoir or lake and is expressed in acre-feet. Volume is computed on the basis of a level pool and does not include bank storage.

Time of day is expressed in 24-hour time; for example, 12:30 a.m. is 0030, 1:30 p.m. is 1330. All times noted are Central standard time. 


\title{
FLOODS OF 1967 IN THE UNITED STATES
}

\section{FLOODS OF SEPTEMBER-OCTOBER 1967 IN SOUTH TEXAS AND NORTHEASTERN MEXICO}

\author{
By Elmer E. Schroeder, R. U. Grozier, D. C. Hahl, \\ and A. E. Hulme
}

\begin{abstract}
Floods produced by Hurricane Beulah during September and October 1967 were outstanding because of the magnitude of the stage and discharge and because of the number of river basins affected. Previously known maximum stages were exceeded, at the downstream station, in five river basins in Texas by amounts ranging from 2.7 feet at Guadalupe River near Tivoli to 9.2 feet at Aransas River near Skidmore.

The greatest relative maximum discharge recorded during the storm occurred at Medio Creek near Beeville, where the peak discharge was 4.1 times the previous maximum since 1919 and 6.0 times the magnitude of a regional 50-year flood. The inflow to Lake Corpus Christi was more than 4.5 times the volume of the lake at spillway elevation.

Because of the large volume of fresh-water inflow to bays and estuaries along the Texas coast, the salinity of the water was greatly reduced. Data collected in Nueces-Corpus Christi and Guadalupe-San Antonio Bays show that dilution proceeded rapidly along the line of flow.

Fresh-water inflow to Corpus Christi Bay exceeded 60,000 cubic feet per second from September 23 through September 28. The total inflow was about 1.5 times the volume of water normally in the bay, but because of its shape and depth, the bay was not entirely flushed of saline water.

Fresh-water inflow to San Antonio Bay exceeded 40,000 cubic feet per second from September 21 through September 26. The total inflow was more than three times the volume of water normally in the bay, and most of the saline water was flushed from the bay.
\end{abstract}

Measurements of water levels in wells indicate that Hurricane Beulah caused significant rises in water levels in shallow wells by percolation of rainfall and ponded waters and by the cascading of floodwaters directly into numerous inundated wells.

Flooding along the Rio Grande and its floodways below Falcon Dam was the greatest since the American floodway system was completed in 1926. At Mission Branch Floodway, south of McAllen, Tex., the peak discharge was 2.15 times the previous maximum in 1932. The peak stage exceeded the previous maximum by 4.14 feet. Flooding along the Mexican floodways destroyed all stream-gaging equipment.

A 4,000-square-mile area of south Texas having no defined drainage system contains thousands of shallow wind-formed depressions. These normally dry depressions were inundated by the storm runoff, which produced a vast amount of ponded water. The ponds blocked highways for several days and hampered ranching and oil field operations for 
months after the storm. Rainfall measurements of 25 inches during the period September 19-25, 1967, were common in Texas, and as much as 35 inches was measured in Mexico.

Total damage in 39 counties of Texas was estimated by the Galveston District of the Corps of Engineers to be $\$ 168,844,000$.

\section{INTRODUCTION}

Torrential rainfall produced by Hurricane Beulah caused floods of record-breaking magnitude on many streams in a 50,000-square-mile area of south Texas and northeastern Mexico in September and October 1967. Beulah made landfall near Brownsville about daybreak on September 20,1967, and dissipated in the mountains of northern Mexico on September 22 (fig. 1). Unofficial rainfall measurements during the period September 19-25 ranged up to 34 inches in the Nueces River basin in Texas and up to 35 inches on the Rio Alamo watershed in Mexico. The highest measurement at a regular U.S. Weather Bureau rainfall station was 25.5 inches near Falls City in Karnes County.

Major flooding occurred on the main and tributary streams in the Guadalupe, San Antonio, Mission, Aransas, and Nueces basins and in many of the small coastal basins in Texas; on the Rio Grande and its floodways; and on the Rio Alamo and Rio San Juan in Mexico.

In Mexico, the magnitude of the flood was so great that most of the recording gages were either submerged, destroyed, or were unable to record the total discharges because water overflowed the natural divides and bypassed the gaging stations. At several streamflow stations in Texas, the maximum rate of flow was more than three times the magnitude of a 50-year flood. The greatest relative discharge rate recorded was on Medio Creek, where the peak discharge was six times that of a 50 -year flood.

In the flood area, stage and discharge data were collected at 53 sites in the network of stream-gaging stations that are maintained by the U.S. Geological Survey in cooperation with various State, local, and Federal agencies. Peak stage and discharge data were also obtained at nine miscellaneous reservoir sites in the Escondido Creek watershed near Kenedy and at 11 other miscellaneous sites throughout the flood area. The International Boundary and Water Commission, United States and Mexico, collected data at 16 regular stations in the Rio Grande basin below Falcon Dam. The Ministry of Hydraulic Resources of Mexico collected data at four stations in Mexico in the Rio Grande basin below Falcon Dam. Rainfall data were collected by the U.S. Weather Bureau in the United States. Rainfall data for Mexico were obtained from the International Boundary and Water Commission.

This report was prepared to present all the documented flood data in a comprehensive, and readily available form. The report includes discussion of the storm; tabulations of rainfall data; an isohyetal map; a description of the floods, by basin, in terms of magnitude, frequency, and urban inundation; a damage report; a section on the effect of fresh- 


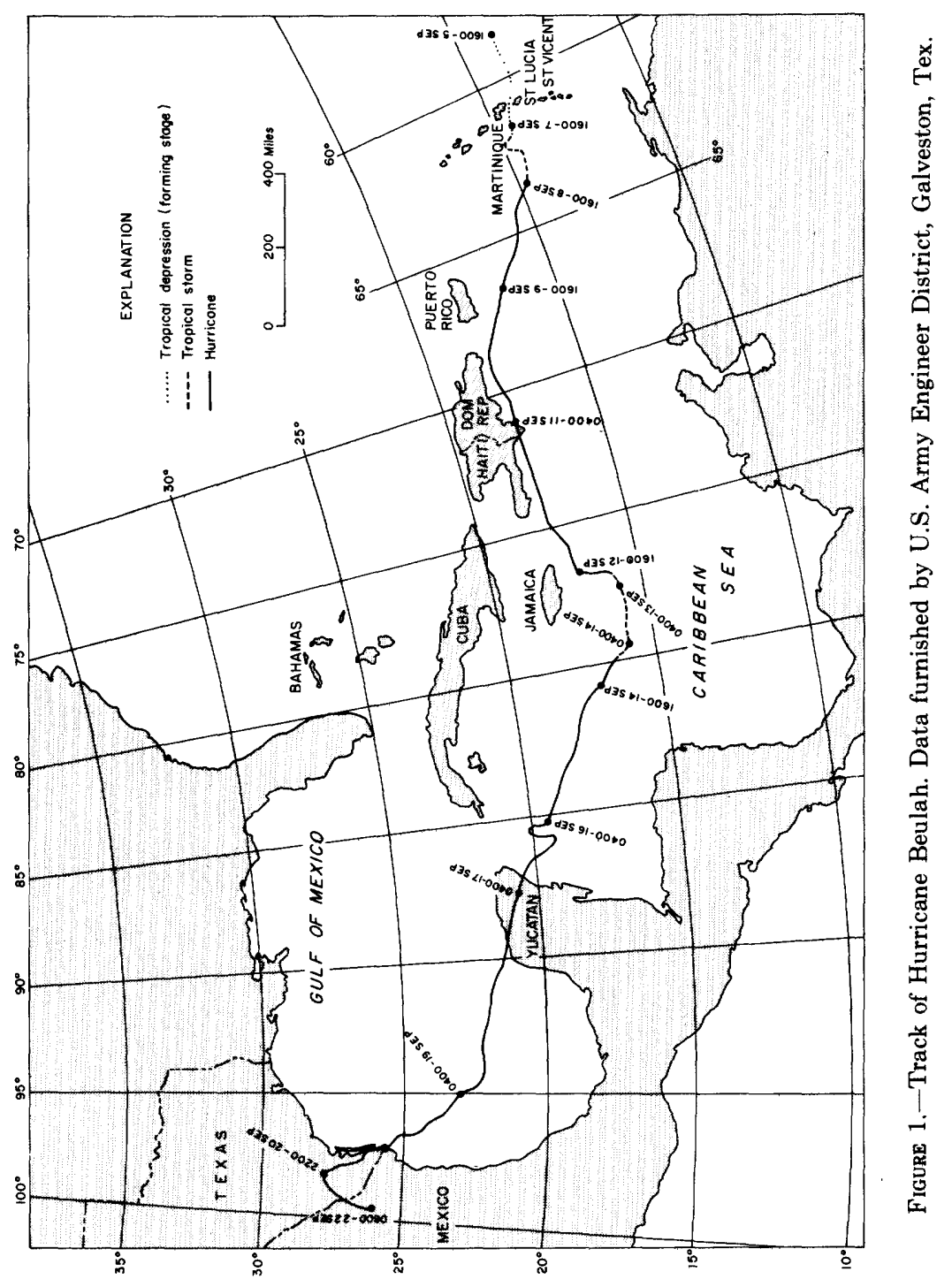


water inflow on the quality of water in Nueces, Corpus Christi, and San Antonio Bays; a discussion of the effect of rainfall on ground-water recharge; a discussion of ponded water on the Coastal Plain; and detailed information on stage, discharge, and maximum rates of discharge for the September-October 1967 period.

\section{ACKNOWLEDGMENTS}

The aid of the many individuals, corporations, and governmental agencies that furnished data and assistance for the preparation of this report is gratefully acknowledged.

The U.S. Weather Bureau assigned men to make precipitation surveys supplementary to their regular rain-gage network; the U.S. Army Corps of Engineers assigned men to make a summary of flood damages; the International Boundary and Water Commission provided information on flood flows and gaging-station records for the Rio Grande and its tributaries; and the Texas Water Development Board provided information on the effects of the floods on ground-water levels.

Collection of field data, necessary for the computation of peak discharge by indirect methods and the calculation of detailed records of discharge, was greatly aided by the work of technical personnel detailed from the U.S. Geological Survey districts of New Mexico, Colorado, Nebraska, and Wyoming. Personnel of the U.S. Geological Survey from Phoenix, Ariz., made aerial photographs of flood crests along many of the rivers. Data furnished by all governmental agencies are specifically acknowledged where they appear in the text.

\section{DESCRIPTION OF THE STORM}

The first advisory on Hurricane Beulah was issued on September 7, 1967. The disturbance was classified as a tropical storm, centered 35 miles west of Martinique in the eastern Caribbean. Winds reached hurricane force about noon on September 8.

As Beulah moved westward across the Caribbean, hurricane winds and rains affected inhabited areas of Martinique, St. Lucia, the Barahona Peninsula of the Dominican Republic, the Tiburon Peninsula of Haiti, and Puerto Rico (fig. 1).

Because part of its circulation was over land, the force of the hurricane diminished, and on September 13, Beulah was downgraded to a tropical storm. By September 14, Beulah regained hurricane force and moved across Cozumel Island and the lowlands of the Yucatan Peninsula. The hurricane entered the Gulf of Mexico on the afternoonof September 17. During the 3 days required for Beulah to reach the coast of south Texas and northeastern Mexico, she gained considerable energy from the warm waters of the Gulf of Mexico.

When Beulah made landfall near Brownsville, Texas (pl. 1), about daybreak on September 20, she was a large, dangerous hurricane with a 
central pressure of 28.05 inches of mercury. A low of 27.25 inches was observed on the previous day by hurricane-hunting aircraft. The maximum wind speed, 136 miles per hour, was reported by the SS Shirley Lykes, which was docked in Port Brownsville. Hurricane-force winds were recorded as far up the coast as Corpus Christi, and as far inland as Alice.

Beulah began to lose windspeed as she moved north-northwest toward Alice. The storm center stalled, then took a southwesterly course and dissipated in the Sierra Madre near Monterrey, Mexico.

During recent years, most of the hurricanes reaching Texas have been captured by the continental weather system, causing them to move rapidly to the north and east and to dissipate their energy over a large area. Beulah moved in the opposite direction and compounded the intensity of energy-mass dissipation by adding an orographic effect to the already torrential rains. Consequently, the greatest amount of rainfall during the storm period occurred on the Rio Alamo watershed, where a maximum of more than 35 inches was reported.

According to reports of the U.S. Weather Bureau, Beulah was the third largest hurricane of record to strike the North American Continent. On the basis of frequency curves (Miller, 1964), the storm produced rainfall in excess of a 100-year recurrence interval for durations of $1-7$ days at a number of stations. Figures 2 and 3 show the accumulated daily rainfall for eight stations where the 2-day, 100-year frequency was exceeded. Figure 4 shows accumulated daily rainfall for five selected stations in Mexico. Although no information on frequency is available for Mexico, the recurrence interval is probably at least 100 years.

Beulah's maximum rainfall intensity was less than that for the storm at Hearne, Tex., on June 28, 1899 (24 inches in 24 hours), or for the storm at Thrall, Tex., on September 9-10, 1921 (38.2 inches in 24 hours), according to U.S. Weather Bureau records. However, a comparison of the areas bounded by the 5 -inch isohyetal lines shows that Beulah was greater in areal extent than either of these storms. Rainfall distribution for the period September 19-25 is shown on plate 1 .

Table 1 lists daily rainfall measurements from regular U.S. Weather Bureau observation stations; table 2 lists supplemental reports from privately owned gages or other receptacles; table 3 lists rainfall data for sites in Mexico, obtained by the U.S. Weather Bureau and the United States' Section of the International Boundary and Water Commission.

Table 4 is a tabulation of accumulated rainfall during the period September 18-25 from a private network of standard rain gages maintained by the four divisions of the King Ranch in Jim Wells, Kleberg, Kenedy, Nueces, and Brooks Counties. The location of these gages is shown in figure 5 . An example of the great variation in rainfall distribution is illustrated by the amounts received at the Encino headquarters 


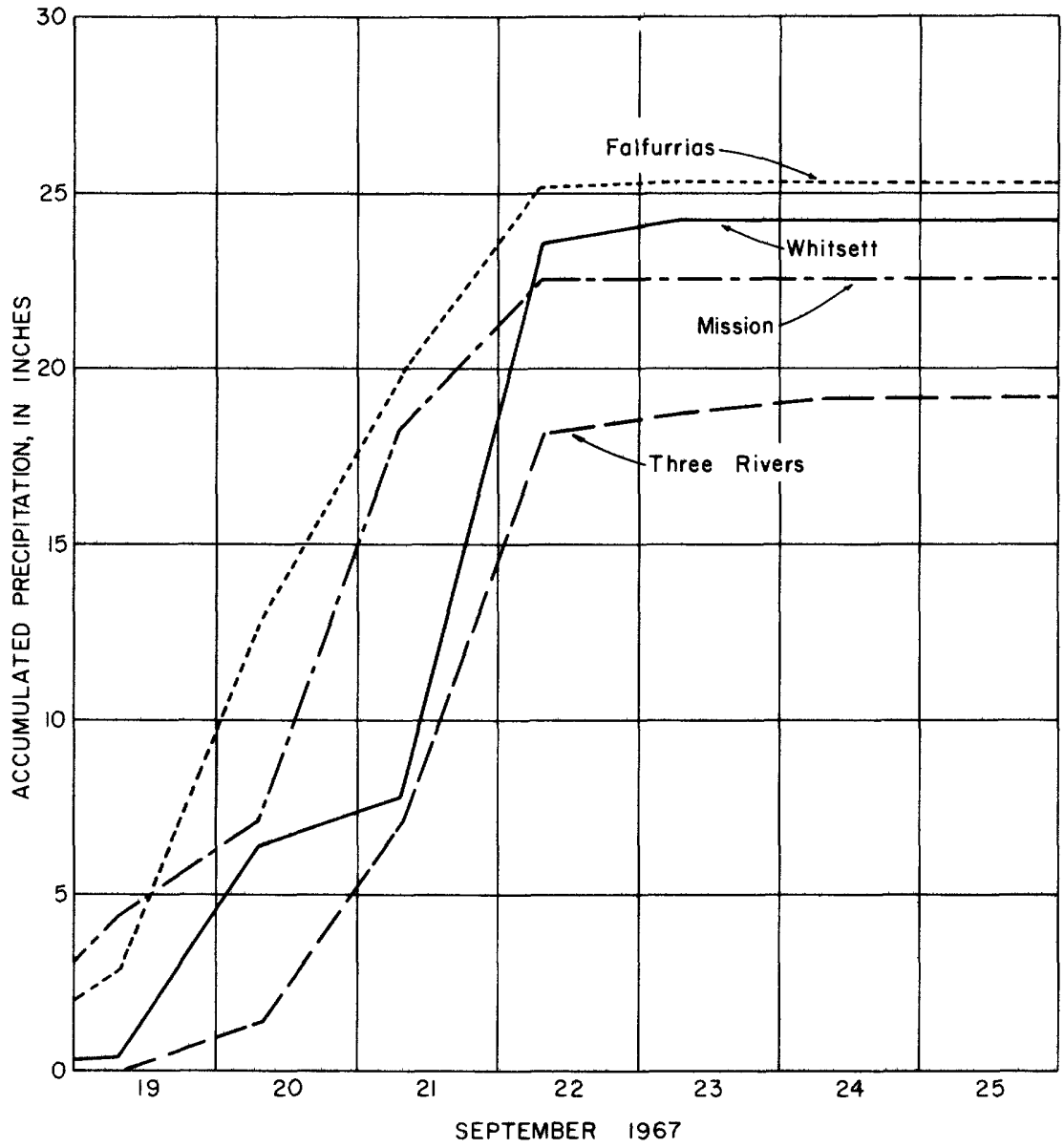

Figure 2.-Accumulation of rainfall at Falfurrias, Whitsett, Mission, and Three Rivers, September 19-25, 1967.

gage and the Encino crossroads gage. A difference of 11.32 inches was measured by these two gages, which are separated by a distance of only 4 miles.

Table 5 is a tabulation of hourly rainfall recorded at 16 U.S. Weather Bureau recording rain gages during the period September 19-25, 1967. The location of these gages is shown in figure 6.

A study of table 5 indicates that hurricane-produced rainfall, frequently referred to as torrential, is not necessarily of record intensity for durations of less than 3 hours. In the list of stations shown in table 5, station 0639 (Beeville $5 \mathrm{NE}$.) recorded the greatest storm total as well as the greatest intensity for durations of 2 to 6 hours. Figures 7 and 8 illustrate how the maximum point rainfall at the Beeville station com- 


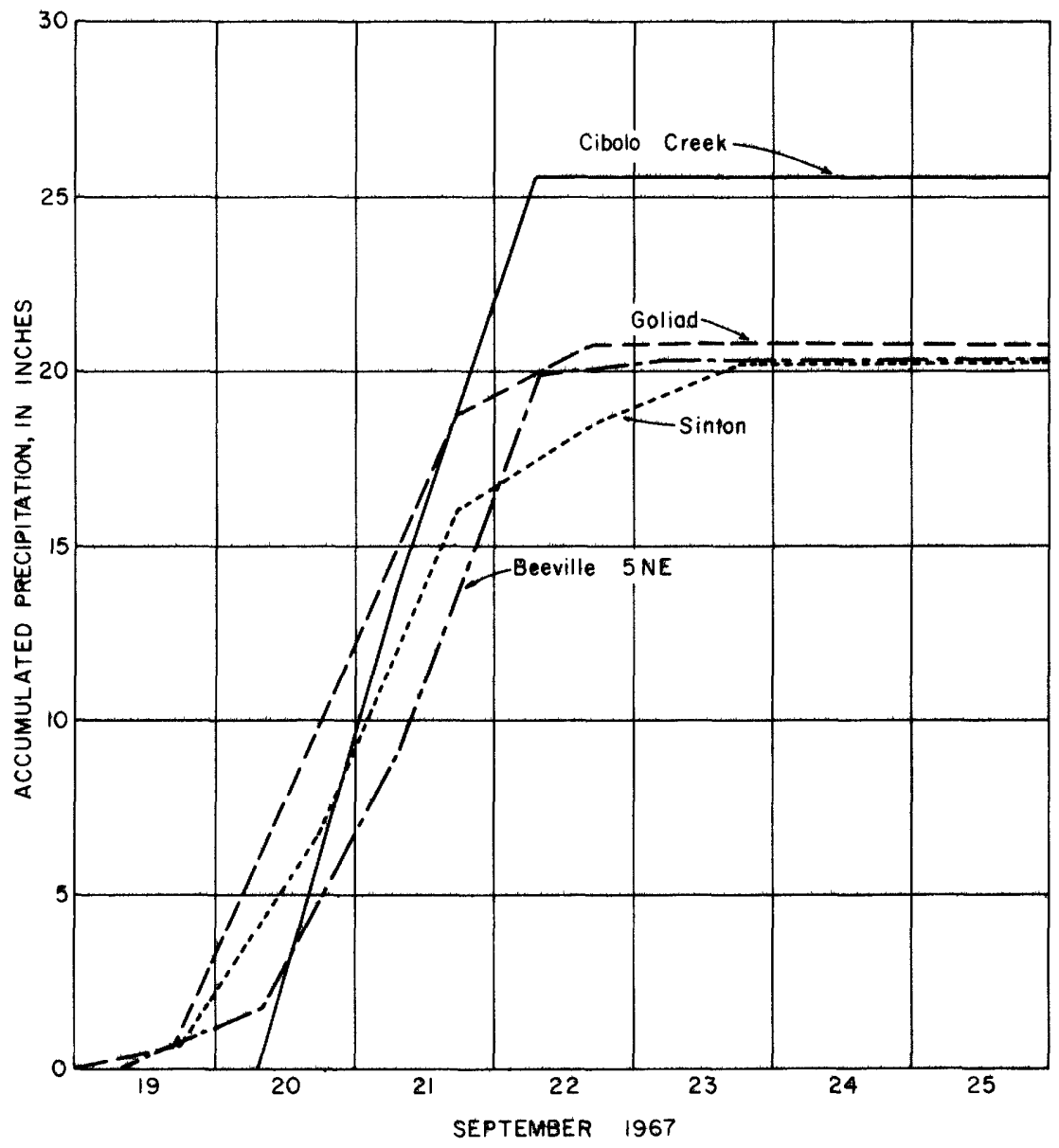

Figux: 3.-Accumulation of rainfall at Cibolo Creek, Goliad, Sinton, and Beeville, September 19-25, 1967.

pares with the intensity-duration-frequency curves for San Antonio and Corpus Christi for durations of 1 to 24 hours.

In addition to large amounts of rainfall, a hurricane also produces high wind velocities, high tides, and low barometric pressures. Figures 9-14 are graphs of barometric pressures and wind velocities for selected weather stations along the gulf coast of Texas. Figures 15-20 are graphs of tide elevations for selected stations along the gulf coast of Texas.

The tide elevation at the Lavaca Bay station (fig. 16) exceeded that at the Matagorda Bay station (fig. 15). This characteristic is rather common in some coestal areas and is the result of land configuration which causes the tide elevation at an inland location to exceed the elevations of both hurricane tides and normal tides. 


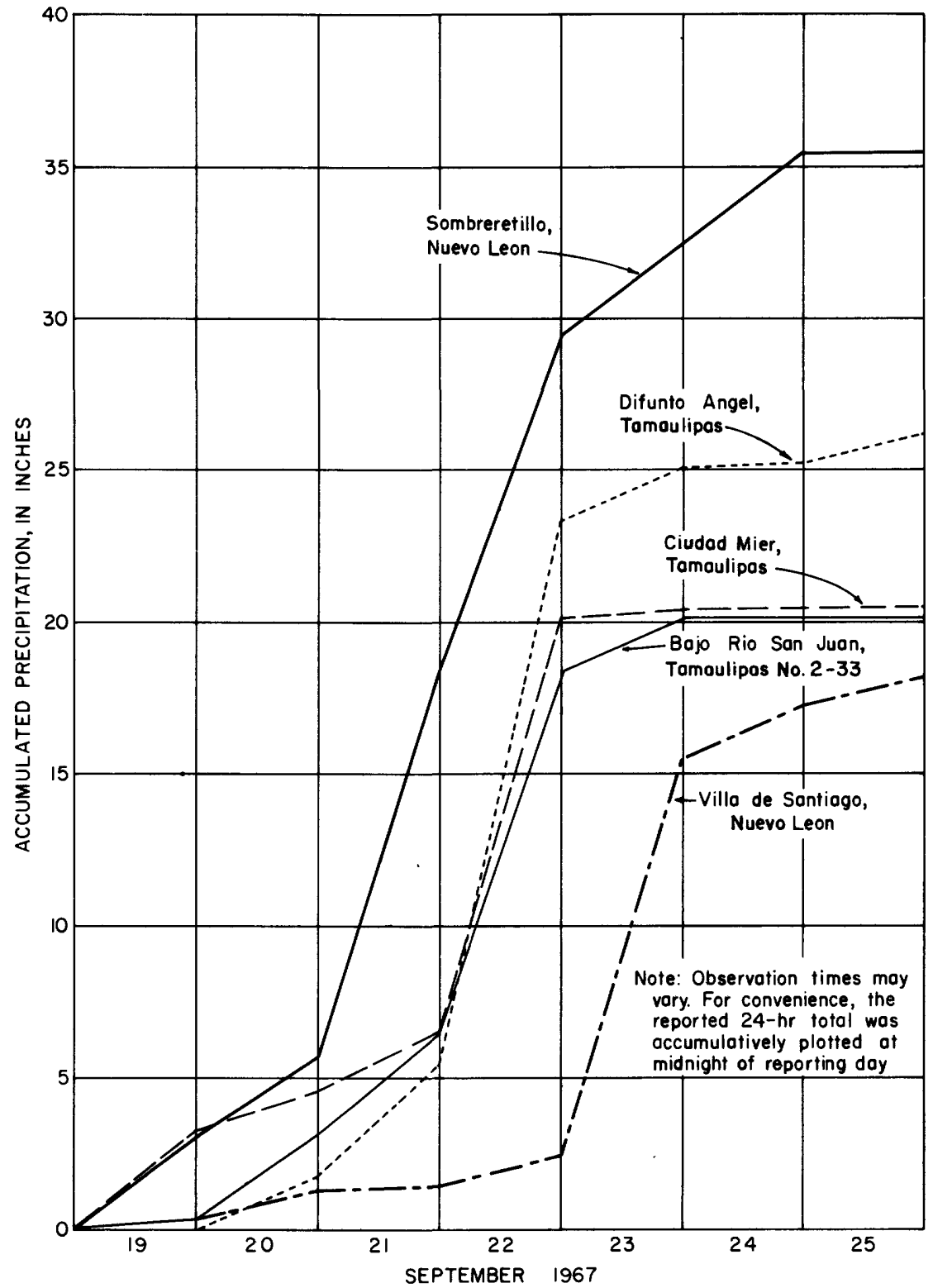

Figure 4.-Accumulation of rainfall at Sombreretillo, Nuevo Leon; Difunto Angel, Tamaulipas; Ciudad Mier, Tamaulipas; Bajo Rio San Juan, Tamaulipas; and Villa de Santiago, Nuevo Leon, Mexico, September 19-25, 1967. 
TABLE 1.-Precipitation, in inches, at U.S. Weather Bureau gages, storm period September 19-25, 1967

* Amount included in following measurement, time distribution unknown. -- No record. T Trace.

\begin{tabular}{|c|c|c|c|c|c|c|c|c|}
\hline \multirow[t]{2}{*}{ COUNTY AND STATION } & \multicolumn{7}{|c|}{$\begin{array}{c}\text { PRECIPITATION COLLECTED ON-- } \\
\text { SEPTEMBER }\end{array}$} & \multirow{2}{*}{$\begin{array}{l}\text { TOTAL } \\
\text { FOR } \\
\text { PERIOD }\end{array}$} \\
\hline & 19 & 20 & 21 & 22 & 23 & 24 & 25 & \\
\hline 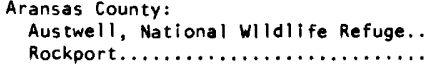 & $\begin{array}{l}1.03 \\
1.55\end{array}$ & $\begin{array}{l}(*) \\
4.50\end{array}$ & $\begin{array}{l}(*) \\
0\end{array}$ & $\begin{array}{r}13.90 \\
.15\end{array}$ & 0.10 & $\begin{array}{l}0 \\
0\end{array}$ & $\begin{array}{l}0 \\
0\end{array}$ & $\begin{array}{l}14.93 \\
12.30\end{array}$ \\
\hline 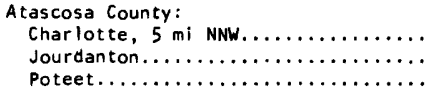 & $\begin{array}{l}.03 \\
.99 \\
.44\end{array}$ & $\begin{array}{l}2.12 \\
4.70 \\
3.15\end{array}$ & $\begin{array}{l}2.50 \\
2.95 \\
2.60\end{array}$ & $\begin{array}{l}5.23 \\
6.72 \\
5.75\end{array}$ & $0_{0}^{.02}$ & $\begin{array}{l}0 \\
0 \\
.50\end{array}$ & $\begin{array}{l}.65 \\
.05 \\
T\end{array}$ & $\begin{array}{l}10.55 \\
15.41 \\
12.44\end{array}$ \\
\hline 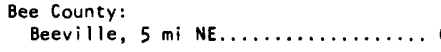 & 0 & 1.87 & 7.43 & 10.61 & .47 & 0 & 0 & 20.38 \\
\hline $\begin{array}{l}\text { Bexar County: } \\
\quad \text { Classens Ranch } \ldots \ldots \ldots \ldots \ldots \ldots \ldots \ldots \\
\text { San Antonio WB Ai rport } \ldots \ldots \ldots \ldots \ldots\end{array}$ & $\begin{array}{l}.39 \\
.31\end{array}$ & $\begin{array}{l}1.91 \\
2.33\end{array}$ & $\begin{array}{l}2.15 \\
2.45\end{array}$ & $\begin{array}{r}1.53 \\
.46\end{array}$ & ${ }^{0} T$ & .26 & $\begin{array}{l}0 \\
0\end{array}$ & $\begin{array}{l}5.98 \\
5.81\end{array}$ \\
\hline $\begin{array}{l}\text { Brooks County: } \\
\text { Falfurrias } \ldots \ldots \ldots \ldots \ldots \ldots \ldots \ldots \ldots\end{array}$ & 2.90 & 10.00 & 7.00 & 5.20 & .20 & $T$ & $\mathbf{T}$ & 25.30 \\
\hline 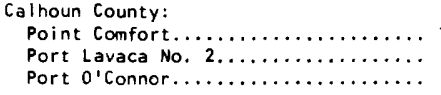 & $\begin{array}{l}1.27 \\
.02\end{array}$ & $\begin{array}{l}7.60 \\
4.36 \\
4.85\end{array}$ & $\begin{array}{l}3.36 \\
7.01 \\
3.60\end{array}$ & $\begin{array}{l}.02 \\
.02 \\
.40\end{array}$ & $\begin{array}{l}0 \\
0 \\
T\end{array}$ & $0_{0}^{.18}$ & $\begin{array}{l}0 \\
0 \\
T\end{array}$ & $\begin{array}{r}12.43 \\
11.41 \\
8.85\end{array}$ \\
\hline 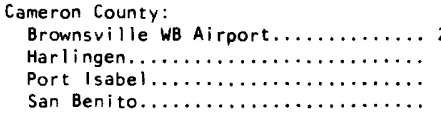 & $\begin{array}{l}2.17 \\
.45 \\
.51\end{array}$ & $\begin{array}{l}12.09 \\
3.85 \\
(*) \\
3.31\end{array}$ & $\begin{array}{l}1.14 \\
4.81 \\
(*) \\
4.35\end{array}$ & $\begin{array}{r}0 \\
16.07 \\
2.23\end{array}$ & .05 & $\begin{array}{l}.69 \\
1.40 \\
0.45\end{array}$ & $0_{0}^{0.06}$ & $\begin{array}{l}16.42 \\
10.49 \\
16.00 \\
10.92\end{array}$ \\
\hline 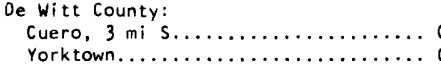 & $\begin{array}{l}0 \\
0\end{array}$ & $\begin{array}{l}1.32 \\
2.00\end{array}$ & $\begin{array}{l}10.90 \\
10.62\end{array}$ & $\begin{array}{l}7.45 \\
9.49\end{array}$ & $\begin{array}{l}.14 \\
.17\end{array}$ & $\begin{array}{l}0 \\
0\end{array}$ & $\begin{array}{l}0 \\
0\end{array}$ & $\begin{array}{l}19.81 \\
22.28\end{array}$ \\
\hline $\begin{array}{l}\text { Duval County: } \\
\text { Benavides, } 3 \text { mi } \$ \ldots \ldots \ldots \ldots \ldots \ldots \ldots \ldots \ldots \ldots \ldots \ldots \ldots \ldots\end{array}$ & $\begin{array}{l}0 \\
0\end{array}$ & $\begin{array}{l}1.15 \\
1.75\end{array}$ & $\begin{array}{r}.60 \\
4.75\end{array}$ & $\begin{array}{l}1.75 \\
5.75\end{array}$ & $\begin{array}{r}.20 \\
3.85\end{array}$ & $\begin{array}{l}0 \\
0\end{array}$ & $0^{.40}$ & $\begin{array}{r}4.10 \\
16.10\end{array}$ \\
\hline $\begin{array}{l}\text { Frio County: } \\
\text { Dilley } \ldots \ldots \ldots \ldots \ldots \ldots \ldots \ldots \ldots \ldots \ldots \ldots \ldots \ldots \ldots \ldots \ldots \ldots\end{array}$ & $\begin{array}{l}0 \\
0\end{array}$ & $\begin{array}{l}.21 \\
1.40\end{array}$ & $\begin{array}{l}2.09 \\
2.23\end{array}$ & $\begin{array}{l}3.81 \\
4.03\end{array}$ & $\begin{array}{l}.68 \\
.19\end{array}$ & $0^{.07}$ & ${ }^{0} .22$ & $\begin{array}{l}6.86 \\
7.85\end{array}$ \\
\hline $\begin{array}{l}\text { Goliad County: } \\
\text { Goliad. } \ldots \ldots \ldots \ldots \ldots \ldots \ldots \ldots \ldots \ldots \ldots \ldots \ldots \ldots\end{array}$ & $.0^{.78}$ & $\begin{array}{l}8.84 \\
1.70\end{array}$ & $\begin{array}{l}9.16 \\
6.56\end{array}$ & $\begin{array}{l}1.96 \\
7.20\end{array}$ & $0^{.05}$ & $0^{\top}$ & $\begin{array}{l}0 \\
0\end{array}$ & $\begin{array}{l}20.79 \\
15.46\end{array}$ \\
\hline 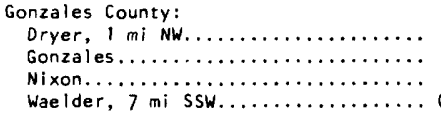 & $\begin{array}{l}.13 \\
.62 \\
.15\end{array}$ & $\begin{array}{l}4.00 \\
1.70 \\
4.25 \\
2.00\end{array}$ & $\begin{array}{l}5.79 \\
6.56 \\
7.24 \\
4.09\end{array}$ & $\begin{array}{l}2.98 \\
7.20 \\
2.55 \\
1.51\end{array}$ & $\begin{array}{l}0 \\
0 \\
0 \\
0\end{array}$ & $\begin{array}{l}0 \\
0 \\
0 \\
0\end{array}$ & $\begin{array}{l}0 \\
0 \\
0 \\
0\end{array}$ & $\begin{array}{r}12.90 \\
16.08 \\
14.19 \\
7.60\end{array}$ \\
\hline 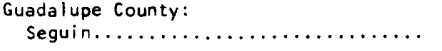 & .21 & 2.96 & 2.95 & 2.05 & 0 & 0 & 0 & 8.17 \\
\hline 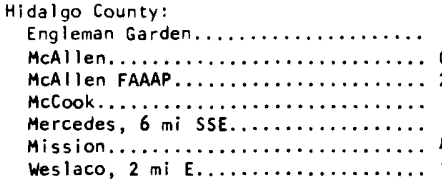 & $\begin{array}{r}.13 \\
0.14 \\
2.78 \\
.09 \\
.40 \\
4.47 \\
1.00\end{array}$ & $\begin{array}{l}4.00 \\
3.06 \\
5.08 \\
4.05 \\
2.44 \\
2.66 \\
2.75\end{array}$ & $\begin{array}{c}3.00 \\
5.52 \\
-- \\
6.28 \\
2.87 \\
11.11 \\
7.33\end{array}$ & $\begin{array}{c}2.75 \\
6.06 \\
-. \\
3.76 \\
2.77 \\
4.46 \\
.40\end{array}$ & $\begin{array}{c}.25 \\
0.87 \\
-. \\
2.42 \\
.54 \\
0.35\end{array}$ & $\begin{array}{c}.25 \\
0.29 \\
-- \\
.44 \\
.21 \\
0.37\end{array}$ & $\begin{array}{l}0 \\
0.11 \\
-. \\
.14 \\
.30 \\
0 \\
0\end{array}$ & $\begin{array}{r}10.38 \\
16.05 \\
-- \\
17.18 \\
9.53 \\
22.70 \\
12.20\end{array}$ \\
\hline $\begin{array}{l}\text { Jackson County: } \\
\text { Edna, } 3 \mathrm{mi} \text { Sw. } \ldots \ldots \ldots \ldots \ldots \ldots \ldots \ldots\end{array}$ & .62 & 6.50 & 5.10 & .05 & 0 & 0 & 0 & 12.27 \\
\hline $\begin{array}{l}\text { Jim Hogg County: } \\
\text { Hebbronville.. } \\
\text { Kaffie Ranch... }\end{array}$ & 2.02 & $\begin{array}{l}1.13 \\
5.50\end{array}$ & $\begin{array}{l}4.00 \\
2.80\end{array}$ & $\begin{array}{l}2.60 \\
3.22\end{array}$ & $\begin{array}{r}7.00 \\
.91\end{array}$ & $0^{.04}$ & $0^{.03}$ & $\begin{array}{l}14.82 \\
14.43\end{array}$ \\
\hline
\end{tabular}


TABLE 1.-Precipitation, in inches, at U.S. Weather Bureau gages, storm period September 19-25, 1967-Continued

COUNTY AND STATION PRECIPITATION COLLECTEO ON--
SEPTEMBER

\section{9}

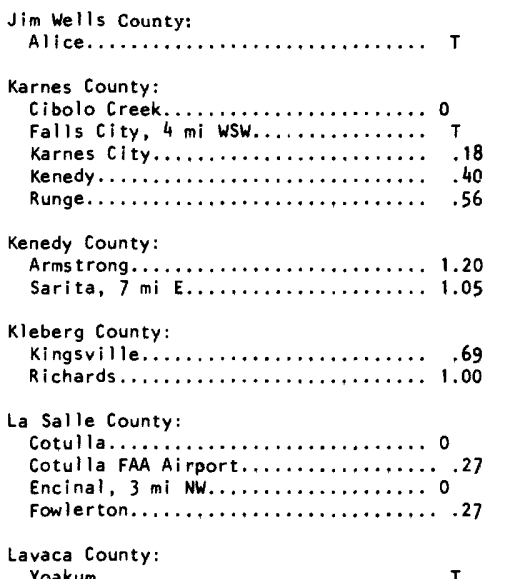

Yoakum...........

George West.

Three Rivers......................

Whitsett, $2 \mathrm{mi}$ SW.............. 40

Matagorda County:

Bay City Waterworks..............
Matagorda No. $2 \ldots \ldots \ldots \ldots \ldots \ldots .52$

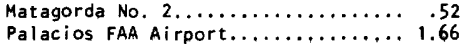

MCMullen County:

Tilden.

Nueces County:

Chapman Ranch............... 1.00

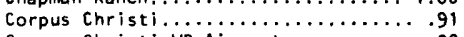

Corpus Christi WB Airport........, .90

Robstown................... .68

Refugio County:

Refugio................... 48

San Patricio County:

Aransas Pass 2................. 1.10

Mathis......................, 0

Sinton................... 82

Welder Wildlife Fndtn........... . 10

Starr County:

Rio Grande City, $3 \mathrm{mi}$ w........ 02

Victoria County:

Victoria WB Airport ........... 1.07

Webb County:

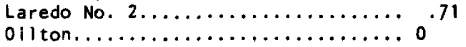

Willacy County:

Port Mansfield.

Raymondville..................... . 09

Wilson County:

floresville.

Zapata County:

Zapata.

\subsection{3}

21

23

24

TOTAL

PERIOO

$\begin{array}{rrrrlll}13.75 & 11.75 & 0 & 0 & 0 & 0 & 25.50 \\ 1.22 & 5.32 & 6.38 & .23 & T & 0 & 13.15 \\ 7.72 & 3.77 & 7.95 & .10 & 0 & 0 & 19.72 \\ 6.62 & 4.19 & 6.90 & .10 & .15 & 0 & 18.36 \\ 5.65 & 6.32 & 6.15 & .02 & 0 & 0 & 18.70\end{array}$

$\begin{array}{llllll}\text { (*) } \quad 11.50 & \text { (*) } \quad 2.80 & \text { (*) } \quad 3.00 \quad 18.50\end{array}$

$\begin{array}{lllllll}3.65 & 5.20 & 2.10 & T & 0 & .76 & 12.76\end{array}$

$\begin{array}{lllllll}3.70 & 3.55 & 4.16 & .04 & 0 & .06 & 12.20\end{array}$

$\begin{array}{lllllll}.70 & 8.00 & 2.36 & .23 & .27 & 0 & 12.56\end{array}$

$\begin{array}{lllllll}.43 & 1.72 & 2.90 & .44 & .09 & 0\end{array}$

$\begin{array}{lllllll}1.78 & 2.38 & 1.58 & .08 & T & 0 & 6.09\end{array}$

$\begin{array}{lllllll}.58 & 1.10 & 4.20 & 1.18 & .04 & 0 & 7.10\end{array}$

$\begin{array}{lllllll}2.20 & 3.20 & 4.50 & .75 & .17 & 0 & 11.09\end{array}$

$\begin{array}{lllllll}4.00 & 3.27 & 0 & 0 & 0 & 0 & 7.27\end{array}$

$\begin{array}{lllllll}1.02 & 5.57 & 9.12 & 1.30 & 1.15 & 0 & 18.16\end{array}$

$\begin{array}{lllllll}1.47 & 5.70 & 11.00 & .75 & .20 & .53 & 19.65\end{array}$

$\begin{array}{lllllll}6.04 & 1.49 & 15.69 & .60 & 0 & 0 & 24.22\end{array}$

$\begin{array}{rllllll}1.20 & 4.88 & 1.25 & 0 & .10 & 0 & 7.33 \\ 3.72 & 6.79 & 0 & 0 & .06 & 0 & 11.09\end{array}$

$\begin{array}{lllllll}3.72 & 6.79 & 0 & 0 & .06 & 0 & 11.09 \\ 6.12 & 3.89 & 0 & T & 0 & 0 & 11.67\end{array}$

$\begin{array}{lllllll}.57 & 3.56 & 6.93 & 1.26 & .62 & .42 & 13.36\end{array}$

$\begin{array}{lllllll}10.00 & 1.50 & 0.50 & 0.85 & 0.30 & 0 & 14.15\end{array}$

$\begin{array}{lllllll}6.76 & 3.68 & 1.26 & .08 & 0 & 0 & 12.69\end{array}$

$\begin{array}{lllllll}3.48 & 6.38 & 3.02 & .29 & 0 & 0 & 14.07\end{array}$

$\begin{array}{lllllll}1.68 & 6.38 & 3.48 & 1.76 & 0 & .24 & 14.22\end{array}$

$\begin{array}{lllllll}7.92 & 4.00 & 0.31 & 0.71 & 0 & 0 & 13.42\end{array}$

$\begin{array}{lllllll}7.38 & 5.10 & 1.60 & .85 & .10 & 0 & 16.13\end{array}$

$\begin{array}{lllllll}1.19 & 8.40 & 7.65 & 2.78 & .24 & 0 & 20.26\end{array}$

$\begin{array}{lllllll}6.05 & 9.16 & 2.60 & 1.66 & .04 & .16 & 20.49\end{array}$

$\begin{array}{lllllll}1.91 & 5.97 & 3.60 & 0 & 0 & 0 & 11.58\end{array}$

$\begin{array}{lllllll}2.42 & 4.00 & 12.51 & 4.96 & T & 1.40 & 25.31\end{array}$

$\begin{array}{lllllll}6.63 & 5.27 & T & 0 & 0 & 0 & 12.97\end{array}$

$\begin{array}{lllllll}.55 & 1.33 & 3.61 & .95 & .10 & 0 & 7.25\end{array}$

$\begin{array}{lllllll}38 & 3.31 & 2.22 & 3.52 & .16 & 0 & 9.59\end{array}$

(*) $\quad$ (*) $\quad 14.30 \quad 0 \quad 00014.68$ $\begin{array}{lllllll}0 & 8.88 & 4.87 & .64 & 1.40 & .32 & 16.20\end{array}$

$\begin{array}{lllllll}3.30 & 2.70 & 9.25 & .50 & 0 & 0 & 15.75\end{array}$

$\begin{array}{lllllll}2.24 & 2.42 & 3.11 & 1.62 & .58 & 0 & 10.44\end{array}$ 
TABLE 2.-Precipitation, in inches, at supplemental sites, storm period September 19-25, 1967

\begin{tabular}{|c|c|c|c|c|c|c|c|c|c|c|}
\hline \multirow[t]{2}{*}{ SITE } & \multirow[t]{2}{*}{ LAT. } & \multirow[t]{2}{*}{ LONG. } & \multicolumn{7}{|c|}{$\begin{array}{l}\text { PRECIPITATION COLLECTED ON-- } \\
\text { SEPTEMBER }\end{array}$} & \multirow{2}{*}{$\begin{array}{l}\text { TOTAL } \\
\text { FOR } \\
\text { PER 10D }\end{array}$} \\
\hline & & & 19 & 20 & 21 & 22 & 23 & 24 & 25 & \\
\hline Alice, $3 \mathrm{mi} E$ & $27^{\circ} 44^{\prime}$ & $98^{\circ} 02^{\prime}$ & 0.64 & 6.65 & 2.35 & 4.79 & 0.20 & 0.01 & 0.01 & 14.65 \\
\hline Calliham, $5 \mathrm{mi} \mathrm{N}$ & $28^{\circ} 33^{\prime}$ & $98^{\circ} 21^{\prime}$ & - & -- & $\cdots$ & $\cdots$ & -- & -- & -- & 16.5 \\
\hline Calliham, $8.5 \mathrm{mi} \mathrm{N}$ & $28^{\circ} 35^{\prime}$ & $98^{\circ} 23^{\prime}$ & 2.25 & 7.90 & 9.60 & .55 & .80 & -- & - & 21.1 \\
\hline Charco, $3 \mathrm{mi} \mathrm{W}$ & $28^{\circ} 44^{\prime}$ & $97^{\circ} 40^{\prime}$ & -. & -- & $\cdots$ & $\cdots$ & - & 21.5 & $-\cdot$ & 21.5 \\
\hline Cuero, $10 \mathrm{mi}$ NNE & $29^{\circ} 13^{\prime}$ & $97^{\circ} 13^{\prime}$ & -- & 5.0 & 5.0 & 2.0 & -- & $\cdots$ & - & 12.0 \\
\hline Falfurrias, $10 \mathrm{mi} \mathrm{s}$ & $27^{\circ} 04^{\prime}$ & $98^{\circ} 09^{\prime}$ & .18 & 2.0 & $6.0+$ & -- & 13.0 & -- & -- & 21.18 \\
\hline Fannin & $28^{\circ} 42^{\prime}$ & $97^{\circ} 14^{\prime}$ & -- & -- & 16.0 & 4.0 & $-\cdot$ & -- & -- & 20.0 \\
\hline Goliad, $10 \mathrm{mi}$ SSE & $28^{\circ} 32^{\prime}$ & $97^{\circ} 21^{\prime}$ & - & -- & - & -- & 19.5 & -- & -- & 19.5 \\
\hline Hochhe im, $4 \mathrm{mi} s$ & $29^{\circ} 11^{\prime}$ & $97^{\circ} 17^{\prime}$ & -- & 2.0 & 8.0 & 4.1 & $-\cdot$ & -- & -- & 14.1 \\
\hline Nursery, $8 \mathrm{mi} \mathrm{W}$ & $28^{\circ} 55^{\prime}$ & $97^{\circ} 14^{\prime}$ & - & $5.0+$ & 10.0 & 4.0 & -- & +- & -- & $19.0+$ \\
\hline Placedo, $6 \mathrm{mi} \mathrm{SE}$ & $28^{\circ} 38^{\prime}$ & $96^{\circ} 45^{\prime}$ & -- & -- & -- & 16.0 & -- & - & - & 16.0 \\
\hline Premont, $2 \mathrm{mi} \mathrm{N}$ & $27^{\circ} 24^{\prime}$ & $98^{\circ} 07^{\prime}$ & - & 3.0 & $6.0+$ & 5.0 & 5.0 & .2 & - & $19.2+$ \\
\hline Realitos, SE & $27^{\circ} 21^{\prime}$ & $98^{\circ} 29^{\prime}$ & -- & -- & -- & -- & -- & 19.5 & -- & 19.5 \\
\hline San Diego & $27^{\circ} 46^{\prime}$ & $98^{\circ} 14^{\prime}$ & -- & - & -- & -- & - & $\cdots$ & 15.8 & 15.8 \\
\hline Stockdale, $1.5 \mathrm{mi} 55 \mathrm{~W}$ & $29^{\circ} 13^{\prime}$ & $97^{\circ} 59^{\prime}$ & - & 4.5 & 4.5 & $4.5+$ & -- & -- & - & $13.5+$ \\
\hline Thomaston & $29^{\circ} 00^{\prime}$ & $97^{\circ} 09^{\prime}$ & -- & -- & +- & 22.00 & - & -- & $\rightarrow$ & 22.00 \\
\hline Tilden, $6 \mathrm{mi} \mathrm{SE}$ & $28^{\circ} 24^{\prime}$ & $98^{\circ} 27^{\prime}$ & -- & 3.3 & 3.9 & 4.1 & - & 1.1 & - & 12.4 \\
\hline Tilden, $8 \mathrm{mis}$ & $28^{\circ} 21^{\prime}$ & $98^{\circ} 34^{\prime}$ & - & -- & -- & $* 19.3$ & - & 1.1 & - & $20.4+$ \\
\hline Victoria & $28^{\circ} 49^{\prime}$ & $97^{\circ} 00^{\prime}$ & -- & -- & 18.0 & -- & -- & $\cdots$ & -- & 18.0 \\
\hline Victoria, $10 \mathrm{mi} \mathrm{NW}$ & $28^{\circ} 56^{\prime}$ & $97^{\circ} 05^{\prime}$ & $\rightarrow$ & -- & -- & 22.2 & - & $-\cdot$ & -- & 22.2 \\
\hline Weesat che & $28^{\circ} 51^{\prime}$ & $97^{\circ} 27^{\prime}$ & -- & -- & $\cdots$ & 21.02 & $\cdots$ & - & -- & 21.02 \\
\hline Wes thoff & $29^{\circ} 11$ & $97^{\circ} 28$ & $\cdots$ & -. & 10.00 & $\omega$ & 10.9 & -- & - & 20.9 \\
\hline Yorktown, $8 \mathrm{mi} \mathrm{SE}$ & $28^{\circ} 53^{\prime}$ & $97^{\circ} 28^{\prime}$ & -- & - & - & $21.0+$ & -- & $\cdots$ & - & $23.0+$ \\
\hline
\end{tabular}

TABLE 3.-Precipitation, in inches, at weather stations in Mexico, storm period September 19-25, 1967

\begin{tabular}{|c|c|c|c|c|c|c|c|c|c|c|c|}
\hline \multicolumn{2}{|c|}{ STATIOM } & LATITUDE & LOMG ITUDE & 19 & \multicolumn{5}{|c|}{$\begin{array}{l}\text { PRECIPITATION CQLLECTED OH-- } \\
\text { SEPTEMEER }\end{array}$} & 25 & $\begin{array}{l}\text { TOTML } \\
\text { Fon } \\
\text { PEnIOS }\end{array}$ \\
\hline \multirow{2}{*}{\multicolumn{2}{|c|}{ AdJuntes, M. L. }} & $25^{\circ} 18^{\prime}$ & $100 \% 00^{\circ}$ & T & $1 . n$ & 0.12 & 1.02 & 12.60 & 1.57 & 2.36 & 19.41 \\
\hline & Agen tlance Camans, M.L. & $25^{\circ} 32^{\circ}$ & $100^{\circ} 30^{\circ}$ & 0.43 & .55 & 2.68 & 4.96 & .98 & .24 & -. & 9.8 \\
\hline \multicolumn{2}{|l|}{ Aqual logues, M.L. } & $26^{\circ} 19^{\prime}$ & $99^{\circ} 32^{\circ}$ & -- & 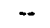 & -- & 6.00 & 6.00 & 6.00 & $\cdots$ & 18.00 \\
\hline \multicolumn{2}{|l|}{ Anohuex. M.L. } & $27^{\circ} 15^{\prime}$ & $100^{\circ} 08^{\circ}$ & $\mathbf{T}$ & .31 & .43 & 2.52 & 1.00 & T & .85 & 5.11 \\
\hline \multicolumn{2}{|l|}{ Apodeca, N.L. } & $25^{\circ} 46^{\prime}$ & $100^{\circ} 11^{\prime}$ & -- & 1.65 & .98 & .83 & 5.33 & 3.62 & 1.28 & 13.69 \\
\hline \multirow[t]{4}{*}{ Bejo Rio travo, Tamps. Ho } & $.1-2$ & $25^{\circ} 56^{\prime}$ & 97.461 & .39 & 3.21 & 4.78 & 4.90 & .39 & .30 & $\cdots$ & 13.89 \\
\hline & $1-3$ & $25^{\circ} 50^{\circ}$ & $97^{\circ} 421$ & .16 & 3.15 & 3.94 & 2.95 & .47 & .31 & .39 & 11.37 \\
\hline & $1-4$ & $25^{\circ} 51^{\circ}$ & 97.451 & .20 & 3.34 & 4.06 & 3.74 & .71 & .55 & - & 12.57 \\
\hline & $1-12$ & $255^{\circ} 56^{\prime}$ & $97^{\circ} 38^{\prime}$ & .79 & 2,48 & 3.23 & 1.46 & .12 & $\cdots$ & 1.18 & 9.26 \\
\hline
\end{tabular}

See footnetes ot end of table. 
TABLF 3.-Precipitation, in inches, at weather stations in Mexico, storm period September 19-25, 1967-Continued

\begin{tabular}{|c|c|c|c|c|c|c|c|c|c|c|c|}
\hline \multirow{2}{*}{\multicolumn{2}{|c|}{ STATION }} & \multirow[t]{2}{*}{ LATITUOE } & \multirow[t]{2}{*}{ LONGITUDE } & \multicolumn{7}{|c|}{$\begin{array}{l}\text { PRECIPITATION COLLECTEO ON-- } \\
\text { SEPTEMBER }\end{array}$} & \multirow{2}{*}{$\begin{array}{l}\text { TOTAL } \\
\text { FOR } \\
\text { PER } 100\end{array}$} \\
\hline & & & & 19 & 20 & 21 & 22 & 23 & 24 & 25 & \\
\hline \multirow[t]{11}{*}{ Bajo Rlo Bravo, Tamps. Mo. } & 13 & $25^{\circ} 44^{\prime}$ & $97^{\circ} 40^{\prime}$ & $-\cdots$ & (*) & 7.09 & 1.77 & -- & -- & -- & 8.86 \\
\hline & & $25^{\circ} 48^{\prime}$ & $97^{\circ} 491$ & .16 & $(*)$ & $(*)$ & 112.60 & -- & -- & .67 & 13.43 \\
\hline & & $25^{\circ} 44^{\prime}$ & $97^{\circ} 53^{\prime}$ & .20 & 1.50 & 1.65 & 4.72 & 1.57 & -- & -- & 9.64 \\
\hline & 7 & $25^{\circ} 39^{\prime}$ & $97^{*} 42$ & -- & $(\star)$ & 6.50 & .98 & .39 & $\cdots$ & $\cdots$ & 7.87 \\
\hline & & $25^{\circ} 40^{\prime}$ & $97^{\circ} 55^{\prime}$ & .12 & $(\star)$ & 5.91 & 4.72 & 1.57 & .16 & .24 & 12.72 \\
\hline & 10 & $25^{\circ} 36^{\prime}$ & $97^{\circ} 52$ & -- & $(\star)$ & 5.39 & 5.35 & 1.50 & .35 & -- & 12.59 \\
\hline & 12 & $25^{\circ} 59^{\prime}$ & $977^{\circ} 38^{\prime}$ & .79 & 2.48 & 3.23 & 1.46 & .12 & -- & 1.18 & 9.26 \\
\hline & 14 & $25^{\circ} 56^{\prime}$ & $97^{\circ} 591$ & .26 & 2.05 & 3.03 & 1.63 & .81 & 1.56 & .06 & 9.40 \\
\hline & 15 & $25^{\circ} 46^{\prime}$ & $98^{\circ} 01^{\prime \prime}$ & .24 & 1.97 & 2.64 & 2.95 & 1.77 & .43 & .39 & 10.39 \\
\hline & 17 & $25^{\circ} 49^{\prime}$ & $97^{\circ} 58^{\prime}$ & .28 & $(\star)$ & $(*)$ & 10.24 & .79 & .79 & .20 & 12.30 \\
\hline & 16 & $25^{\circ} 35^{\prime}$ & $98^{\circ} 00^{\circ}$ & .24 & 3.54 & 3.15 & 2.40 & 2.56 & .67 & .31 & 12.87 \\
\hline \multirow[t]{9}{*}{ Bajo Rio San Juan, Tamps. No. } & $2-29$ & $26^{\circ} 10^{\prime}$ & $98^{\circ} 38^{\prime}$ & -- & 2.95 & 3.94 & 6.50 & 2.58 & .10 & 1.36 & 17.43 \\
\hline & $2-33$ & $26^{\circ} 10^{\prime}$ & $98^{\circ} 28^{\prime}$ & .16 & 2.95 & 3.23 & 12.20 & 1.57 & $\cdots$ & - & 20.11 \\
\hline & $2-38$ & $26^{\circ} 06^{\prime}$ & $98^{\circ} 34^{\prime}$ & -- & .30 & 1.57 & 3.35 & 5.53 & 1.77 & - & 12.52 \\
\hline & $3-42$ & $26^{\circ} 04^{\prime}$ & $98^{\circ} 19^{\prime}$ & .13 & 2.85 & 5.05 & 4.79 & 1.29 & - & 1.52 & 15.63 \\
\hline & $3-47$ & $25^{\circ} 58^{\prime}$ & 98.071 & -- & 1.77 & 4.64 & 3.87 & 2.30 & .24 & .17 & 12.99 \\
\hline & $3-55$ & $25^{\circ} 52^{\prime}$ & $98^{\circ} 12^{\prime}$ & $\cdots$ & 5.91 & 3.54 & 2.13 & .20 & - & $\cdots$ & 11.78 \\
\hline & $3-58$ & $25^{\circ} 50^{\prime}$ & $98 \cdot 11$ & - & 4.33 & 5.12 & 2.95 & 2.05 & .79 & .47 & 15.71 \\
\hline & $3-60$ & $25^{\circ} 46^{\prime}$ & $98^{\circ} 10^{\prime}$ & $\cdots$ & 4.53 & 5.04 & 3.27 & 1.85 & .98 & .51 & 16.18 \\
\hline & $?-63$ & $25^{\circ} 41^{\prime}$ & $98^{\circ} 06^{\star}$ & $\cdots$ & 4.53 & 4.72 & 3.74 & 1.57 & .35 & .28 & 15.19 \\
\hline \multicolumn{2}{|l|}{ Barranco Azul, Tamps. } & $24^{\circ} 24^{\prime}$ & $99^{\circ} 07^{\prime}$ & $-\cdot$ & 1.39 & .85 & 2.18 & 3.37 & 1.55 & .39 & 9.73 \\
\hline \multicolumn{2}{|l|}{ Brecha Arguelles, Tamps. } & $26^{\circ} 11^{\prime}$ & $98^{\circ} 28 \cdot$ & -- & 2.95 & 3.23 & 12.20 & 1.57 & +- & -- & 19.95 \\
\hline \multicolumn{2}{|l|}{ Burges, Tamps. } & $24^{\circ} 57^{\prime}$ & $98^{\circ} 48 \cdot$ & .31 & 2.56 & .79 & 4.33 & 3.27 & 1.77 & .20 & 13.23 \\
\hline \multicolumn{2}{|l|}{ Bustamante, N.L. } & $26^{\circ} 32^{\prime}$ & $100^{\circ} 30^{\prime}$ & -. & 2.20 & .98 & 4.53 & 4.17 & .55 & .08 & 12.51 \\
\hline \multicolumn{2}{|l|}{ Cabezones, M.L. } & $24^{\circ} 59^{\prime}$ & $99^{\circ} 45^{\prime}$ & .28 & 1.54 & .14 & .48 & 4.50 & 1.37 & 99. & 9.30 \\
\hline \multicolumn{2}{|l|}{ Cadereyta, N.L. } & $25^{\circ} 35^{\prime}$ & $100^{\circ} 00^{\prime}$ & -- & 1.83 & .16 & 3.07 & 2.96 & 1.71 & $\cdots$ & 9.73 \\
\hline \multicolumn{2}{|l|}{ Camargo, Tamps. } & $26^{\circ} 19^{\prime}$ & $98^{\circ} 50^{\prime}$ & .08 & 1.57 & 3.54 & 9.57 & 1.38 & .06 & -- & 16.20 \\
\hline \multicolumn{2}{|l|}{ Candela, cosh. } & $26^{\circ} 51^{\circ}$ & $100^{\circ} 40^{\prime}$ & -- & 1.02 & .83 & 4.80 & 5.83 & .47 & -- & 12.95 \\
\hline \multicolumn{2}{|l|}{ Co, Mier, Tamps. } & $26^{\circ} 26^{\prime}$ & $99^{\circ} 09$ & 3.15 & 1.38 & 1.97 & 13.58 & .31 & -- & - & 20.39 \\
\hline \multicolumn{2}{|l|}{ Cerralvo, N.L. } & $26^{\circ} 05^{\prime}$ & $99^{\circ} 37^{\prime}$ & .04 & .94 & .58 & 9.70 & 2.05 & -- & -- & 13.31 \\
\hline \multicolumn{2}{|l|}{ Cerritos, N.L. } & $25^{\circ} 31^{\circ}$ & $100^{\circ} 12^{\prime}$ & .12 & 1.06 & .71 & 2.99 & 12.60 & 1.50 & .87 & 19.85 \\
\hline \multicolumn{2}{|l|}{ Cerro Prieto, N.L. } & $24^{\circ} 56^{\prime}$ & $99^{\circ} 23^{\prime}$ & .35 & -- & -- & .83 & $\cdots$ & 2.13 & 1.93 & 5.24 \\
\hline China, N.L. & & $25^{\circ} 42^{\prime}$ & $99^{\circ} 14+$ & -- & 1.54 & 1.38 & 9.29 & 1.38 & -- & .31 & 13.90 \\
\hline Clenega de Flores, N.L. & & $25^{\circ} 57^{\circ}$ & $100^{\circ} 10^{\prime}$ & 0.20 & 1.41 & 0.71 & 8.07 & 3.66 & 0.72 & $r$ & 14.77 \\
\hline Comales, Tamps. & & $26^{\circ} 111$ & $98^{\circ} 55^{\prime}$ & -- & 1.71 & 3.52 & 11.89 & 2.27 & .10 & -- & 19.49 \\
\hline Control, Tamps. & & $25^{\circ} 18^{\prime}$ & $977^{\circ} 49$ & $\cdots$ & $(*)$ & $(*)$ & 9.00 & .24 & .20 & .08 & 9.52 \\
\hline Cruillas, Tamps. & & $24^{\circ} 45^{\prime}$ & $98^{\circ} 32^{\prime}$ & .31 & 3.43 & .79 & 1.73 & 7.32 & 1.77 & 1.57 & 16.92 \\
\hline Difunto Angel, Tamps. & & $26^{\circ} 23^{\prime}$ & $99^{\circ} 021$ & -- & 1.94 & 3.64 & 17.71 & 1.78 & .18 & .88 & 26.13 \\
\hline Div. de Munic, Y Canal Rode, & Tamps. & $26^{\circ} 06^{\prime}$ & $98^{\circ} 34^{\prime}$ & -- & .30 & 1.57 & 3.35 & 5.51 & 1.79 & -- & 12.52 \\
\hline Don Martin, Coah. & & $27^{\bullet} 31$ & $100^{\bullet} 37$ & -- & .08 & .20 & 1.73 & .93 & .33 & .04 & 3.31 \\
\hline El Barrote, Tamps. & & $26 \cdot 10$ & $98^{\circ} 38^{\prime}$ & -- & 2.95 & 3.94 & 6.50 & 2.58 & .10 & 1.36 & 17.43 \\
\hline E) Cuchillo, N.L. & & $25^{\circ} 43^{\prime}$ & $99^{\circ} 16^{\prime}$ & .08 & .67 & .68 & 9.29 & 1.36 & 2.81 & .58 & 15.47 \\
\hline EI Manzano, M.L. & & $25^{*} 32^{\prime}$ & $100^{\circ} 28^{\prime}$ & .08 & 2.72 & .12 & 1.30 & 13.11 & 2.76 & 1.30 & 21.39 \\
\hline El Ocotillal, Tamps. & & $24^{\circ} 24^{\prime}$ & $98^{\circ} 28^{\prime}$ & .24 & .65 & .51 & .18 & 5.91 & 2.40 & .22 & 10.11 \\
\hline El Pealito. N.L. & & $25^{\circ} 18^{\prime}$ & $99^{\circ} 211^{\prime \prime}$ & 5.16 & 2.83 & .35 & 7.48 & .55 & 2.05 & .39 & 18.81 \\
\hline Estacion C amacho, N.L. & & 24.531 & $99^{\circ} 35^{\prime}$ & .49 & .75 & .15 & .50 & 2.72 & .96 & .35 & 5.92 \\
\hline
\end{tabular}


TABLE 3.-Precipitation, in inches, at weather stations in Mexico, storm period September 19-25, 1967-Continued

\begin{tabular}{|c|c|c|c|c|c|c|c|c|c|c|}
\hline \multirow{2}{*}{ STATION } & \multirow{2}{*}{ LATITUDE } & \multirow{2}{*}{ LONGITUDE } & \multicolumn{5}{|c|}{$\begin{array}{l}\text { PRECHPITATION COLLECTEO O } \\
\text { SEPTEMBER }\end{array}$} & \multicolumn{2}{|l|}{ ON=- } & \multirow{2}{*}{$\begin{array}{l}\text { TOTAL } \\
\text { FOR } \\
\text { PERIOD }\end{array}$} \\
\hline & & & 19 & 20 & 21 & 22 & 23 & 24 & 25 & \\
\hline Garza Ayala, N.L. & $26^{\circ} 29^{\prime}$ & $100^{\circ} 03^{\prime}$ & -- & 2.20 & .81 & 2.36 & 2.38 & 1.21 & -- & 8.96 \\
\hline Gral. Bravo, N.L. & $25^{\circ} 48^{\prime}$ & $99^{\circ} 11^{\prime}$ & .08 & 1.73 & 1.52 & 11.79 & .87 & 1.42 & .41 & 17.82 \\
\hline Gral. Trevino, N.L. & $26^{\circ} 14^{4}$ & $99^{\circ} 29^{\prime}$ & .20 & .79 & - & -- & 9.84 & $-\cdot$ & $-\cdot$ & 10.83 \\
\hline Hedionda Grande, Coah. & $25^{\circ} 07^{\prime}$ & $100^{\circ} 51^{\prime}$ & .67 & .39 & .28 & .51 & -- & $\cdots$ & -- & 1.85 \\
\hline Hidalgo, Tamps. & $24^{\circ} 15^{\prime}$ & $99^{\circ} 26^{\prime}$ & .02 & .04 & .27 & .36 & 3.84 & 1.59 & .43 & 6.55 \\
\hline Higue ras, N.L. & $25^{\circ} 58^{\circ}$ & $100^{\circ} 01^{\prime}$ & 1.06 & 1.42 & 11.81 & 2.83 & .67 & $-\cdot$ & $-\cdot$ & 17.79 \\
\hline inst. Nac. Invest. Agr., W.L. & $25^{\circ} 18^{\prime}$ & $99^{\circ} 36^{\prime}$ & .12 & 2.20 & .16 & 2.52 & 1.97 & 1.77 & 1.57 & 10.31 \\
\hline Iturbide, N.L. & $24^{\circ} 44^{\prime}$ & $99^{\circ} 54^{\prime \prime}$ & .58 & .69 & .02 & 1.02 & 3.76 & .70 & .23 & 7.00 \\
\hline Jimenez, Tamps. & $24^{\circ} 13^{\prime}$ & $98^{\circ} 29^{\prime}$ & .09 & .59 & .41 & .17 & 8.78 & 4.56 & .20 & 14.80 \\
\hline La Arena, N.L. & $25^{\circ} 46^{\prime}$ & $100^{\circ} 01^{\prime}$ & - & .73 & .39 & 10.86 & 2.23 & 2.03 & .81 & 17.05 \\
\hline La cruz, N.L. & $25^{\circ} 28^{\prime}$ & $100^{\circ} 26^{\prime}$ & .47 & .63 & 2.99 & 5.20 & .91 & .12 & -- & 10.32 \\
\hline La Encarnacion, Tamps. & & & 4.24 & 1.38 & .26 & 3.98 & 5.81 & 7.09 & .28 & 23.04 \\
\hline La Gloria, N.L. & $26^{\circ} 53^{\prime}$ & $99^{\circ} 49^{\prime}$ & 1.97 & 2.17 & 1.57 & 2.36 & - & -- & $\cdots$ & 8.07 \\
\hline Laguna de Sanchez, N.L. & $25^{\circ} 21^{\prime}$ & $100^{\circ} 17^{\prime}$ & .08 & 1.38 & .31 & 1.83 & 6.69 & 1.65 & .65 & 12.59 \\
\hline La Popa, N.L. & $26^{\circ} 10^{\prime}$ & $100^{\circ} 50^{\circ}$ & -- & 1.42 & 1.38 & 2.09 & 1.89 & -- & -- & 6.78 \\
\hline Las Comitas, M.L. & & & .79 & -- & 1.89 & .55 & 3.23 & .61 & .67 & 7.74 \\
\hline Las Enramadas, N.L. & $25^{\circ} 30^{\prime}$ & $99^{\circ} 31^{\prime}$ & $\top$ & 2.76 & .59 & 7.30 & 3.35 & 1.97 & + & 15.97 \\
\hline Las Norias, Tamps. & $24^{\circ} 37^{\prime}$ & $98^{\circ} 18^{\prime}$ & .79 & 2.48 & .87 & 1.38 & 9.31 & 2.00 & .25 & 17.08 \\
\hline Linares, N.L. & $24^{\circ} 52$ & $99^{\circ} 34^{\prime}$ & .49 & .75 & .15 & .50 & 2.72 & .96 & .35 & 5.92 \\
\hline Los Aldanas, N.L. & $26^{\circ} 04^{\prime}$ & $99^{\circ} 12^{\prime}$ & -- & $\cdots$ & 4.13 & 5.91 & $\cdots$ & $\cdots$ & -- & 10.04 \\
\hline Los Herreras, N.L. & $25^{\circ} 54^{\prime}$ & $99^{\circ} 24^{\prime}$ & .30 & 1.65 & .57 & 10.63 & 3.15 & - & -- & 16.30 \\
\hline Los Ramones, N.L. & $25^{\circ} 42^{\prime}$ & $99^{\circ} 38^{\prime}$ & .16 & 1.26 & .16 & 6.30 & 1.89 & 1.89 & .31 & 11.97 \\
\hline Magueyes, Tamps. & $24^{\circ} 34^{\prime}$ & $99^{\circ} 33^{\prime}$ & .36 & .33 & .09 & .65 & 4.33 & 1.04 & 1.00 & 1.80 \\
\hline Hendez, Tamps. & $25^{\circ} 07^{\prime}$ & $98^{\circ} 35^{\prime}$ & $\top$ & 1.97 & .24 & 3.74 & 8.46 & 1.97 & 1.06 & 17.44 \\
\hline Migue i Aleman, Tamps. (same as Difunto, Tamps.) & $26^{\circ} 24^{\prime}$ & $99^{\circ} 02^{\prime}$ & -- & 1.94 & 3.64 & 17.71 & 1.78 & .18 & .86 & 2613 \\
\hline Mina, M.L. & $26^{\circ} 00^{\prime}$ & $100^{\circ} 32^{\prime}$ & -- & .75 & .69 & 4.21 & .71 & .55 & .12 & 7.03 \\
\hline Monclova, Coah. & $26 \cdot 54 \cdot$ & $101.25^{\prime}$ & .10 & 1.20 & 1.84 & 4.23 & .72 & .07 & $-\cdot$ & 8.16 \\
\hline Montemorelos, N.L. & $25^{\circ} 12^{\prime}$ & $99^{\circ} 50^{\prime}$ & .04 & 1.57 & .14 & 1.02 & 6.89 & 1.10 & .04 & 10.80 \\
\hline Monterrey, N.L. & $25^{\circ} 40^{\prime}$ & $100^{\circ} 18^{\circ}$ & $\cdots$ & 1.54 & $T$ & 2.13 & 5.63 & 1.16 & .26 & 10.72 \\
\hline Muzqulx, Coah. & $27^{*} 53^{\prime}$ & $101^{\circ} 31^{\circ}$ & $-\cdot$ & 0.35 & 0.51 & 2.48 & 1.73 & -- & 0.08 & 5.15 \\
\hline Nueve Cd. Guerrero, Tamps. & $26^{\circ} 34^{\prime}$ & $99^{\circ} 14^{\circ}$ & -. & .69 & 1.69 & 12.80 & 2.01 & .08 & .37 & 17.64 \\
\hline Nuevo Laredo, Jamps. CILA & $27^{*} 30^{\circ}$ & $99^{\circ} 30^{\circ}$ & - & .55 & 1.69 & 3.27 & 1.65 & .20 & .20 & 7.56 \\
\hline Pajonal, N.L. & $25^{\circ} 29^{\prime}$ & $100^{\circ} 23^{\prime}$ & $\cdots$ & 1.57 & .59 & 2.56 & 3.98 & .79 & .79 & 10.28 \\
\hline Paso del Aura, Tamps. & & & 1.77 & 1.91 & .91 & 3.94 & 5.51 & .79 & .59 & 15.42 \\
\hline Potrero Redondo, N.L. & $25^{\circ} 16^{\circ}$ & $100^{\circ} 10^{\circ}$ & .04 & .59 & 1.18 & 2.36 & 2.76 & 2.68 & 3.74 & 13.35 \\
\hline Progreso, Cosh. & $27^{\circ} 25^{\prime}$ & $101^{\circ} 00^{\prime \prime}$ & 1.18 & .43 & 1.93 & 2.24 & .08 & $\cdots$ & $\cdots$ & 5.86 \\
\hline Rancho Mercedes, Coah. & $28^{\circ} 02^{\prime}$ & $100^{\circ} 01^{\prime}$ & .39 & .12 & $-\cdot$ & .71 & .31 & - & .63 & 2.16 \\
\hline Rancho San Juan de la Palma, Tamps. & $26^{\circ} 54^{\prime}$ & $99^{\circ} 20^{\prime}$ & -- & .83 & 2.24 & 3.82 & 1.57 & 1.18 & -- & 9.64 \\
\hline Rayones, M.L. & $25^{\circ} 01^{\prime}$ & $100^{*} 05^{\prime}$ & .39 & .83 & $T$ & .87 & 1.18 & 1.14 & 1.16 & 5.57 \\
\hline Reynosa, Tamps. & $26^{\circ} 06^{\prime}$ & $98^{\circ} 17^{\prime}$ & .20 & 3.94 & 7.48 & 3.15 & 1.06 & $\cdots$ & -- & 15.83 \\
\hline Reynosa Km. 40, SW, N.L. & $25^{\circ} 57^{\prime}$ & $98^{\circ} 39^{\prime}$ & .94 & 7.09 & 5.51 & 5.12 & 2.36 & .71 & 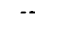 & 21.73 \\
\hline Rinconada, N.L. & $25^{\circ} 41^{\prime}$ & $100^{\circ} 43^{\prime}$ & .24 & .79 & .63 & 4.72 & 1.10 & $\cdots$ & - & 7.48 \\
\hline Rlo Bravo, Tamps. & $25^{\circ} 59^{\prime}$ & $98^{\circ} 06^{\prime}$ & $-\cdot$ & 7.48 & 5.91 & 3.56 & .98 & .31 & .20 & 18.44 \\
\hline Sabinas Hidalgo, M.L. & $26^{\circ} 30^{\prime}$ & $100^{\circ} 10^{\circ}$ & .08 & 1.61 & .79 & 3.31 & 4.06 & .59 & ${ }^{\top}$ & 10.44 \\
\hline San Carlos, Tamps. & $24^{\circ} 35^{\prime}$ & $98^{\circ} 56^{\circ}$ & .37 & 3.78 & 1.36 & 2.99 & 6.61 & 1.54 & .11 & 16.76 \\
\hline San Fernando, Tamps. & $24^{\circ} 511$ & $98^{\circ} 14$ & .91 & 2.48 & .91 & .79 & 4.98 & 2.56 & 2.05 & 14.68 \\
\hline San Juan, M.L. & $25^{\circ} 33^{\prime}$ & $99^{\circ} 50^{\prime}$ & .83 & 3.17 & 1.61 & .83 & 1.42 & .16 & .53 & 8.55 \\
\hline
\end{tabular}


TABLE 3.-Precipitation, in inches, at weather stations in Mexico, storm period September 19-25, 1967-Continued

\section{STATION}

San Miguel de Camergo, Tamps.

San Nicolas, Tamps.

Sante Catarina, N.L.

Santa Teresa, Couh.

sombraretillo, N.L.

Tenacitas, Tamps.

Topo Chico, N.L.

Tunel san Frenciseo. N.L.

valadeces, Tamos.

Vallectllo, N.L.

Villa Ailende. N.L.

Villa de santlago, N.L.

villa Hidalge, Cosh.

\begin{tabular}{|c|c|c|c|c|c|c|c|c|c|}
\hline \multirow{2}{*}{ LATITUDE } & \multirow{2}{*}{ LONG ITUDE } & \multicolumn{7}{|c|}{$\begin{array}{l}\text { PRECIPITATION COLLECTED ON-- } \\
\text { SEPTEMGER }\end{array}$} & \multirow{2}{*}{$\begin{array}{c}\text { TOTAL } \\
\text { FOR } \\
\text { PER }\end{array}$} \\
\hline & & 19 & 20 & 21 & 22 & 23 & 24 & 25 & \\
\hline $26^{\circ} 14^{\circ}$ & $98^{\circ} 36^{\prime}$ & .12 & 3.19 & 2.95 & 2.30 & 1.57 & $\cdots$ & .43 & 10.56 \\
\hline $24^{\circ} 42^{\circ}$ & $98^{\circ} 50^{\prime}$ & .. & ו9. & 7.09 & 1.28 & 1.75 & 5.61 & 1.52 & 18.16 \\
\hline $25 \cdot 40^{\prime}$ & $100^{\circ} 28^{\circ}$ & +- & .87 & .12 & 1.81 & 6.10 & .87 & .24 & 10.01 \\
\hline $26^{\circ} 27^{\prime}$ & $101^{\circ} 24^{\prime}$ & -- & .79 & .20 & 1.85 & 2.13 & .24 & $\cdots$ & 5.21 \\
\hline $26^{\circ} 18^{\prime}$ & $99^{\circ} 58^{\prime}$ & 3.15 & 2.76 & 12.60 & 11.02 & 2.90 & 3.15 & $\cdots$ & 35.67 \\
\hline $25^{\circ} 59^{\prime}$ & $98^{\circ} 02^{\prime}$ & 2.24 & 3.25 & .51 & .33 & 13.58 & 6.38 & .37 & 26.66 \\
\hline $25^{\circ} 44^{\prime}$ & $100^{\circ} 20^{\prime}$ & $\cdots$ & 1.52 & .10 & 2.46 & 4.76 & .79 & .28 & 9.91 \\
\hline $25^{\circ} 25^{\prime}$ & $100^{\circ} 10^{\prime}$ & .09 & 1.33 & .. & - & 8.66 & .02 & .75 & 10.85 \\
\hline $26^{\circ} 14^{\prime}$ & $98^{\circ} 40^{\prime}$ & .06 & 3.25 & 3.56 & 4.40 & 2.05 & $\cdots$ & 1.06 & 14.38 \\
\hline $26^{\circ} 40^{\prime}$ & 99.591 & 1.81 & .08 & .10 & .49 & .24 & .93 & 1.46 & 5.11 \\
\hline $25 * 17$ & $100^{\circ} 01^{\circ}$ & .20 & 1.81 & .20 & 1.10 & 9.92 & 1.06 & .71 & 15.00 \\
\hline $25^{\circ} 25^{\prime}$ & $100^{\circ} 09^{4}$ & .17 & 1.07 & .08 & 1.20 & 13.08 & 1.73 & .79 & 18.12 \\
\hline $27^{\circ} 47^{\prime}$ & $99^{\circ} 52^{\prime}$ & .59 & .55 & 4.57 & 1.18 & .24 & .. & $\cdots$ & 7.13 \\
\hline
\end{tabular}

* Muownt included in following messurement, time distribution unknown.

$\mathrm{T}_{\mathrm{ABLE}}$ 4.-Precipitation, in inches, at King Ranch rain gages, storm period September $18-25,1967$

\begin{tabular}{|c|c|c|c|}
\hline Site & \multicolumn{2}{|c|}{ Site No. } & Total for period \\
\hline \multicolumn{4}{|c|}{ Senta Gertrudis } \\
\hline 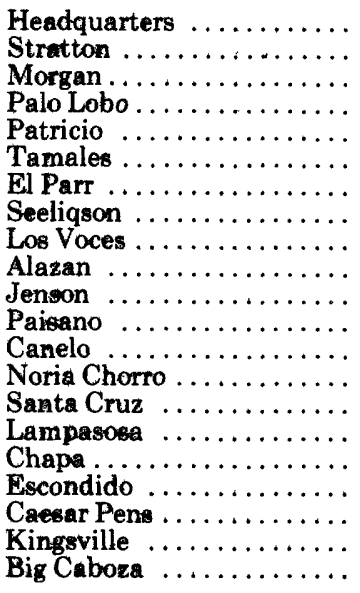 & $\begin{array}{r}1 \\
2 \\
3 \\
4 \\
5 \\
6 \\
7 \\
8 \\
9 \\
10 \\
11 \\
12 \\
13 \\
14 \\
15 \\
16 \\
17 \\
18 \\
19 \\
20 \\
21\end{array}$ & 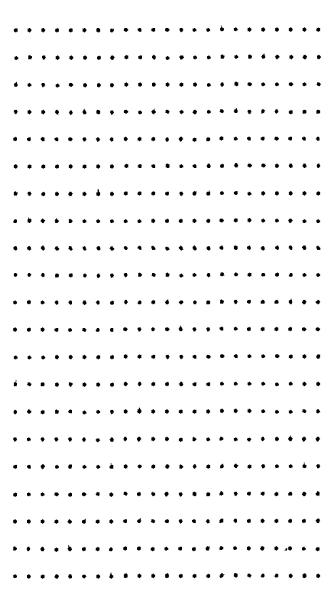 & $\begin{array}{l}13.07 \\
16.12 \\
14.80 \\
13.00 \\
13.61 \\
15.58 \\
15.38 \\
15.78 \\
14.55 \\
14.90 \\
14.82 \\
18.14 \\
13.24 \\
17.00 \\
12.23 \\
15.05 \\
12.77 \\
14.02 \\
12.47 \\
13.56 \\
16.45\end{array}$ \\
\hline \multicolumn{4}{|c|}{ Lawreles } \\
\hline 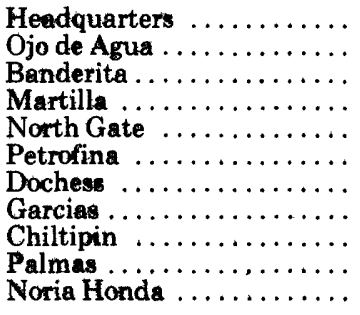 & $\begin{array}{l}22 \\
23 \\
24 \\
25 \\
26 \\
27 \\
28 \\
29 \\
30 \\
31 \\
32\end{array}$ & 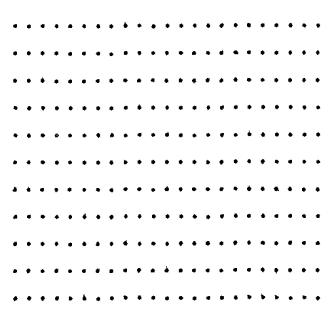 & $\begin{array}{r}12.96 \\
12.65 \\
12.50 \\
12.17 \\
10.16 \\
15.00 \\
11.00 \\
11.15 \\
9.90 \\
5.70 \\
4.00\end{array}$ \\
\hline
\end{tabular}


TABLE 4.-Precipitation, in inches, at King Ranch rain gages, storm period September 18-25, 1967-Continued

\begin{tabular}{|c|c|c|c|}
\hline Site & Site No. & & Total for period \\
\hline \multicolumn{4}{|c|}{ Laureles - Continued } \\
\hline $\begin{array}{l}\text { Kingsville Gate } \ldots \ldots \ldots \ldots \\
\text { Leoncitas } \ldots \ldots \ldots \ldots \ldots \ldots \\
\text { Binotera } \ldots \ldots \ldots \ldots \ldots \\
\text { Telephone } \ldots \ldots \ldots \ldots \ldots \\
\text { Paso Las Flacas } \ldots \ldots \ldots \ldots \\
\text { Jaboncillas } \ldots \ldots \ldots \ldots \ldots \\
\text { Viboras } \ldots \ldots \ldots \ldots \ldots \\
\text { Portales Verde } \ldots \ldots \ldots \ldots \ldots \\
\text { Zacahuistal } \ldots \ldots \ldots \ldots \ldots \\
\text { Alazan } \ldots \ldots \ldots \ldots \ldots \ldots\end{array}$ & $\begin{array}{l}33 \\
34 \\
35 \\
36 \\
37 \\
38 \\
39 \\
40 \\
41 \\
42\end{array}$ & 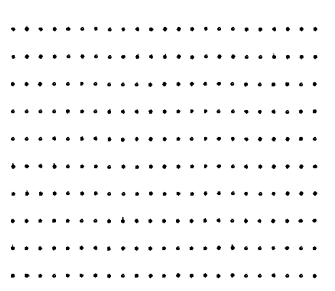 & $\begin{array}{r}8.24 \\
9.05 \\
9.95 \\
12.20 \\
9.30 \\
9.96 \\
10.00 \\
12.60 \\
9.85 \\
9.35\end{array}$ \\
\hline \multicolumn{4}{|c|}{ Norias } \\
\hline $\begin{array}{l}\text { Old Norias } \ldots \ldots \ldots \ldots \ldots \ldots \\
\text { Maravillas } \ldots \ldots \ldots \ldots \ldots \ldots \ldots \\
\text { Stillman } \ldots \ldots \ldots \ldots \ldots \ldots \ldots \\
\text { Calandria } \ldots \ldots \ldots \ldots \ldots \ldots \\
\text { Horacio } \ldots \ldots \ldots \ldots \ldots \ldots\end{array}$ & $\begin{array}{l}43 \\
44 \\
45 \\
46 \\
47\end{array}$ & 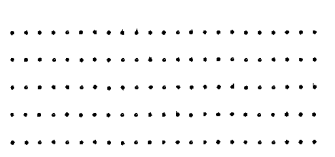 & $\begin{array}{l}16.15 \\
14.00 \\
15.00 \\
20.00 \\
19.40\end{array}$ \\
\hline \multicolumn{4}{|c|}{ Encine } \\
\hline $\begin{array}{l}\text { Headquarters } \ldots \ldots \ldots \ldots \ldots \\
\text { Mineral Exp } \ldots \ldots \ldots \ldots \ldots \ldots \\
\text { Pita } \ldots \ldots \ldots \ldots \ldots \ldots \ldots \\
\text { Venada } \ldots \ldots \ldots \ldots \ldots \ldots \\
\text { Barrosa } \ldots \ldots \ldots \ldots \ldots \ldots \ldots \\
\text { Las Oratis } \ldots \ldots \ldots \ldots \ldots \ldots \\
\text { No. } 1 \text { well } \ldots \ldots \ldots \ldots \ldots \ldots \\
\text { Cross Roads } \ldots \ldots \ldots \ldots \ldots\end{array}$ & $\begin{array}{l}48 \\
49 \\
50 \\
51 \\
52 \\
53 \\
54 \\
55\end{array}$ & 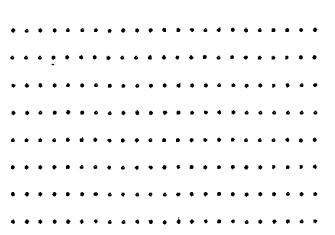 & $\begin{array}{l}21.52 \\
21.10 \\
10.85 \\
15.00 \\
18.05 \\
17.00 \\
17.00 \\
10.20\end{array}$ \\
\hline
\end{tabular}

Figures 19 and 20 illustrate a case of opposite characteristics. The hurricane tide in Corpus Christi Bay at the tidal basin was reduced in magnitude when it reached the Naval Air Force Station. This is probably an exception rather than the rule in the bay systems of the Texas gulf coast.

A unique feature of Hurricane Beulah was the record number of tornadoes (115 and 1 waterspout) spun off from the main storm. Sightings were reported as far up the coast as Houston and as far inland as Austin, where several buildings were slightly damaged by one tornado that touched down within the city. The most severe tornado occurred at Palacios on Matagorda Bay, where three persons were killed, and five were injured.

\section{DESCRIPTION OF THE FLOODS}

Flooding varied from minor to "maximum known" during September-October 1967 in south Texas and northeastern Mexico. From the Lavaca River to the Rio Grande, all streams that discharge into the Gulf of Mexico were affected by the storm. The record-breaking 
$\mathrm{T}_{\mathrm{ABLE}}$ 5.-Hourly precipitation, in inches, at U.S. Weather

ITr.,

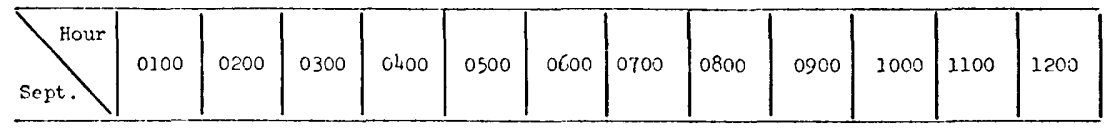

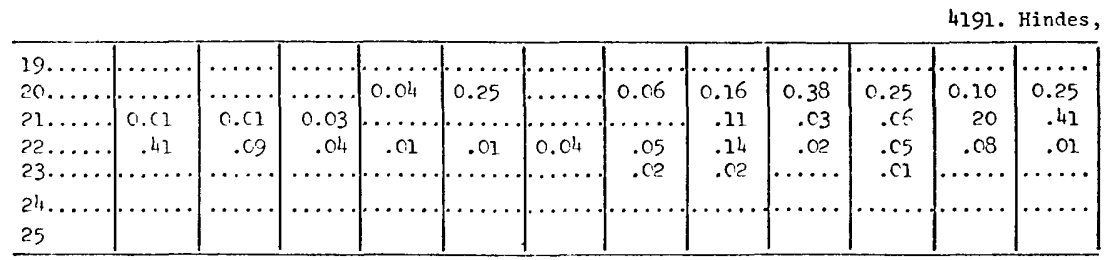

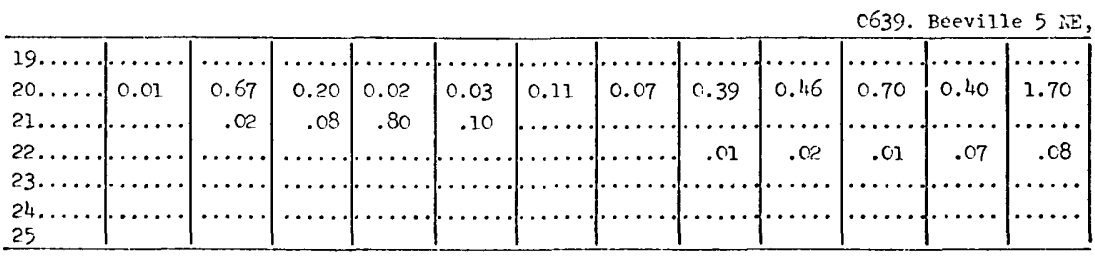

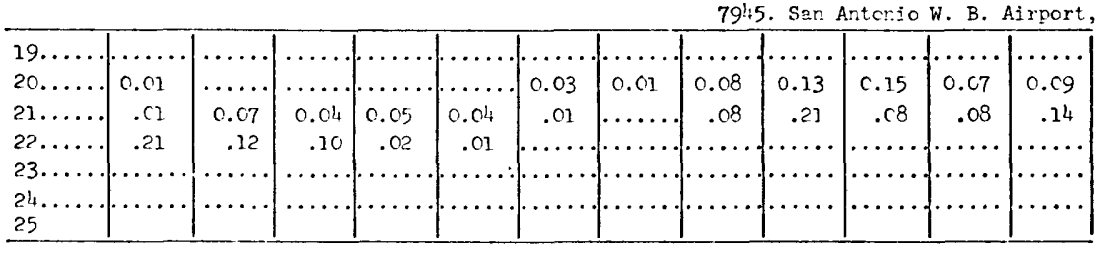

7422. Randolph Field,

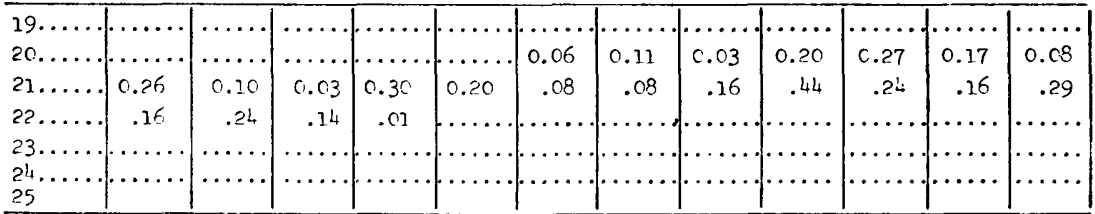

71LC. Poir.t Cowfort.

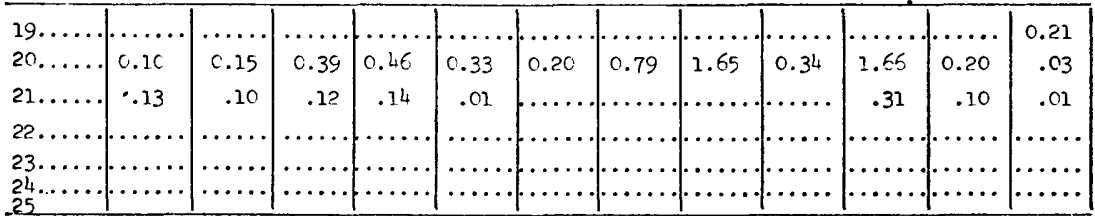

1136. Brownsville w. B. Airport,

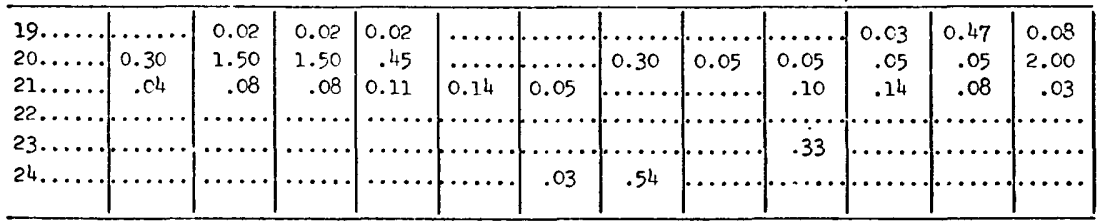

See footnotes at end of table. 


\section{Bureau weighing rain gages, storm of September 19-25, 1967}

\section{tracel}

\begin{tabular}{|c|c|c|c|c|c|c|c|c|c|c|c|c|c|}
\hline 1300 & 1400 & 1500 & 1600 & 1700 & 1800 & 1900 & 2000 & 2100 & 2200 & 2300 & 2400 & $\begin{array}{c}\text { Daily } \\
\text { total } \\
\text { (inches) }\end{array}$ & $\begin{array}{c}\text { Storm } \\
\text { total } \\
\text { (inches) }\end{array}$ \\
\hline
\end{tabular}

Atascosa County

\begin{tabular}{|c|c|c|c|c|c|c|c|c|c|c|c|c|c|}
\hline . & $\ldots$ & $\ldots$ & C.24 & 0.01 & $\ldots$ & & $\ldots$ & $\cdots$ & $\ldots$ & & & 0.25 & \\
\hline$\therefore 20$ & $c .45$ & 1.07 & .18 & .35 & 0.09 & 0.01 & 0.01 & 0.02 & 0.01 & 0.30 & 0.15 & 4.34 & \\
\hline .14 & .45 & .54 &.$? ?$ & .18 & .14 & .12 & .24 & .35 & .30 & .15 & .15 & 3.84 & \\
\hline .40 & .05 & $\ldots \ldots$ & .. & & & . & .03 & .01 & .01 & $\ldots$ & $\ldots$ & 1.45 & \\
\hline. $\mathrm{Cl}$ & $\ldots$ & $\ldots$ & $\ldots$ & $\cdots$ & & . & $\ldots$ & & . & . & $\cdots$ & .05 & $\ldots \ldots$ \\
\hline$\ldots$ & $\ldots \ldots$ & $\ldots \ldots$ & $\ldots$ & & & & . & & & & & & $\ddot{9.94}$ \\
\hline
\end{tabular}

Bee County

\begin{tabular}{|c|c|c|c|c|c|c|c|c|c|c|c|c|c|}
\hline b.c6 & 0.38 & 0.01 & $\ldots$ & $\cdots \cdots$ & 0.01 & 0.01 & & & & & & 0.47 & \\
\hline .35 & .65 & .30 & $0 . c^{4}$ & 0.01 & .14 & .11 & 0.13 & 0.52 & 0.56 & 0.04 & & 7.61 & \\
\hline .11 & .20 & .61 & .45 & 1.65 & 1.94 & 1.66 & 2.10 & 1.35 & .20 & .11 & 0.02 & 11.41 & $\ldots$ \\
\hline $.0 ?$ & .03 & .01 &. $\mathrm{Cl}$ & .04 &. $\mathrm{Cl}_{1}$ & $\cdots$ & - & $\cdots$ & $\cdots$ & & & .35 & $\cdots$ \\
\hline$\cdots \cdots$ & $\cdots \cdots$ & - & $\ldots$ & $\ldots \ldots$ & $\ldots$ & $\cdots$ & & & 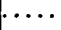 & & & . & $\ldots$ \\
\hline$\ldots \ldots \ldots$ & $\cdots \cdots$ & $\cdots$ & $\cdots \cdots$ & $\cdots \cdots$ & $\cdots \ldots$ & $\cdots \cdots$ & $\ldots$ & $\cdots$ & $\ldots$ & & & & $\ddot{19}$ \\
\hline
\end{tabular}

Bexar County

\begin{tabular}{|c|c|c|c|c|c|c|c|c|c|c|c|c|c|}
\hline$\cdots \cdots$ & $\ddot{\cdots} \cdot$ & $\cdots \cdots$ & $\cdots$ & 0.12 & $\ldots$ & $\cdots$ & -2 & 0.11 & 0.08 & $\cdots \cdots$ & $\cdots \cdots$ & 0.31 & \\
\hline 0.24 & 0.15 & c. 11 & 0.30 & .75 & 0.01 & 0.05 & 0.05 & $\ldots$ & .06 & $\ldots$. & 0.14 & 2.33 & $\cdots$ \\
\hline .02 & .02 & .04 &. $\mathrm{Cl}$ & .05 & .14 & .20 & .32 & .32 & .20 & 0.17 & .15 & 2.45 & * \\
\hline$\cdots \ldots$ & $\cdots \cdots$ & $\cdots$ & & & & 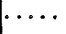 & 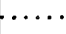 & & . & *. & $\cdots \cdots$ & 46 & \\
\hline$\cdots \cdots$ & $\cdots \cdots$ & $\cdots \cdots$ & $\cdots$ & $\cdots$ & $\cdots$ & & $\cdots$ & $\cdots$ & $\cdots \cdots$ & $\cdots \cdots$ & $\cdots \cdots$ & $\ldots \ldots$ & $\cdots$ \\
\hline$\cdots$ & $\cdots$ & $\cdots \cdots$ & $\cdots \cdots$ & . . & $\cdots \cdots$ & $\cdots \cdots$ & $\cdots$ & $\cdots$ & $\ldots \ldots$ & $\cdots \cdots$ & $\cdots \cdots$ & $\cdots$ & $\begin{array}{l}\ldots .55 \\
5.5\end{array}$ \\
\hline
\end{tabular}

Bexar Courity

\begin{tabular}{|c|c|c|c|c|c|c|c|c|c|c|c|c|c|}
\hline & $\ldots$ & & & c.c7 & c.ce & & & & 0.81 & 0.05 & $\ldots$ & 1.01 & \\
\hline 0.10 & 0.65 & c. .10 & c. 20 & $.4 c$ & $.3 C$ & $\ldots$ & 0.09 & 0.01 & .05 & .01 & $\ldots \ldots$ & 2.83 & \\
\hline .01 &.$C ?$ &.$C 2$ & .01 & .20 & .34 & 0.42 & .43 & .30 & .30 & .28 & C.22 & 4.89 & \\
\hline$\cdots$ & $\ldots$ & $\ldots \ldots$ & $\ldots$ & $\ldots \ldots$ & $\ldots$ & $\ldots$ & $\ldots$. & $\ldots$ & $\ldots$ & $\ldots$ & $\ldots$ & .55 & • . . \\
\hline & . & & & & & & & $\cdots \cdots$ & $\cdots \cdots$ & $\ldots \ldots$ & & $\cdots$ & \\
\hline & $\cdots$ & $\ldots$ & $\cdots$ & $\cdot$ & . & & $\ldots$ & $\ldots \ldots$ & . & $\ldots$ & & & 9 \\
\hline
\end{tabular}

Calion Courty

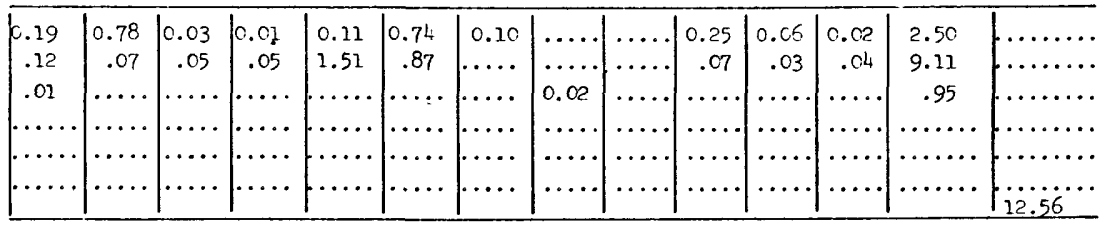

Cameron County

\begin{tabular}{|c|c|c|c|c|c|c|c|c|c|c|c|c|c|}
\hline 0.12 & 0.07 & $\ldots \ldots$ & 0.01 & 0.01 & 0.01 & 0.03 & 0.54 & 0.23 & 0.31 & 0.10 & 0.10 & 2.17 & \\
\hline 2.00 & 2.00 & 0.30 & .30 & .30 & .29 & .22 & .11 & .07 & .14 & .11 & $\ldots \ldots$ & 12.09 & \\
\hline$\ldots \ldots$ & .06 & .01 & .02 & .02 & .10 & .07 & $\ldots \ldots$ & .01 & $\cdots$ & $\cdots$ & $\ldots$ & 1.14 & \\
\hline$\cdots \cdots$ & $\cdots$ & $\cdots$ & $\cdots$ & & 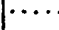 & & $\cdots \cdots$ & $\cdots$ & $\cdots$ & $\cdots$ & $\cdots$ & $\cdots \cdots$ & $\cdots$ \\
\hline$\cdots$ & & & & & & & $\ldots \ldots$ & $\ldots$ & & $\ldots$ & . & .33 & $\cdots$ \\
\hline .12 & & & & & & & & & & & & .69 & \\
\hline
\end{tabular}


TABLE 5.-Hourly precipitation, in inches, at U.S. Weather

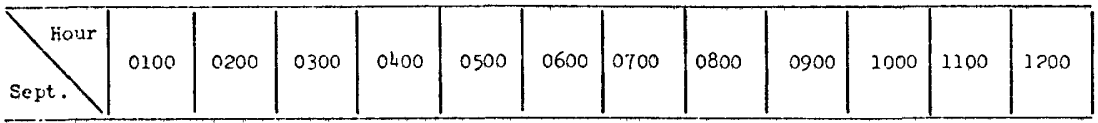

0689. Benavides

\begin{tabular}{|c|c|c|c|c|c|c|c|c|c|c|c|c|}
\hline 19. & & & & & & & & $\cdots$ & $\cdots$ & & $\ldots \ldots$ & $\cdots$ \\
\hline 20. & $\ldots \ldots$ & 0.01 & $0.1 ?$ & 0.04 & 0.01 & 0.06 & 0.23 & 0.26 & 0.06 & 0.10 & 0.53 & 0.52 \\
\hline 21. & 0.01 & .10 & .05 & .05 & .11 & $.0 ?$ & .22 & .20 & .22 & .25 & .12 & .26 \\
\hline 22. & .06 & .06 & .13 & .01 & .35 & .55 & .2 .4 & .52 & .33 & .05 & .07 & .23 \\
\hline 23. & $\ldots \ldots$ & ..... & $\ldots \ldots$ & .97 & .61 & .12 & .02 & $\cdots \cdots$ & $\cdots \cdots \cdots$ & $\ldots \ldots$ & $\cdots$ & .... \\
\hline $\begin{array}{l}21_{4} \\
25\end{array}$ & $\ldots$ & . & $\cdots$ & $\cdots$ & $\cdots$ & $\cdots$ & - & $\cdots \cdots$ & $\ldots \ldots$ & $\ldots \ldots$ & $\ldots \ldots$ & .38 \\
\hline
\end{tabular}

16\%1. Cheepside,

\begin{tabular}{|c|c|c|c|c|c|c|c|c|c|c|c|c|}
\hline 29. & & & & & & & & & & & ...... & \\
\hline 20. & 0.01 & 0.01 & 0.11 & 0.16 & 0.10 & 0.08 & 0.26 & 0.16 & 0.19 & 0.19 & 0.25 & 1.02 \\
\hline $21 .$. & .10 & .28 & .16 & .27 & .17 & .15 & .12 & .58 & .90 & .25 & .23 & .10 \\
\hline 22. & .02 & .01 & $\ldots \ldots$ & $\ldots$ & & & $\cdots$ & ....... & $\ldots \ldots$ & $\ldots \ldots$ & $\ldots \ldots$ & $\cdots \cdots$ \\
\hline $\begin{array}{l}23 . \\
24 . \\
25\end{array}$ & & $\begin{array}{l}\cdots \cdots \\
\cdots \cdots\end{array}$ & & & & & & & & & $\begin{array}{l}\cdots \cdots \\
\cdots \cdots\end{array}$ & $\cdots \cdots$ \\
\hline
\end{tabular}

9588. Welasco 2E,

\begin{tabular}{|c|c|c|c|c|c|c|c|c|c|c|c|c|}
\hline $19 . . .$. & ..... & ..... & & & & & & & & 0.01 & 0.11 & 0.59 \\
\hline $2 c \ldots \ldots$ & $\ldots .$. & 0.05 & 0.15 & 0.19 & 0.06 & 0.15 & 0.45 & 0.24 & 0.02 & .28 & .11 & .12 \\
\hline $21 \ldots \ldots$ & 0.55 & .46 & .39 & .08 & .04 & .08 & .10 & 2.05 & 1.10 & .25 & .10 & .05 \\
\hline $22 \ldots \ldots$ & $\ldots .$. & .04 & .04 & .01 & .01 & & & & $\cdots$ & & & $\cdots$ \\
\hline 23. & & $\ldots \ldots$ & $\cdots \cdots$ & . & & & & & .14 & .02 & & - \\
\hline $\begin{array}{l}2^{4 .} . \\
25\end{array}$ & & $\cdot$ & .... & ..... & & & & & & & & \\
\hline
\end{tabular}

\begin{tabular}{|c|c|c|c|c|c|c|c|c|c|c|c|c|}
\hline 9. & $\ldots \ldots$ & $\ldots \ldots$ & $\ldots \ldots$ & 0.06 & 0.01 & $\ldots \ldots$ & $\cdots \cdots$ & 0.01 & 0.01 & & & \\
\hline .. & 0.01 & 0.12 & 0.01 & 0.03 & c. 10 & 1.15 & 0.25 & .10 & .20 & 0.33 & 0.07 & 0.10 \\
\hline$\ldots \ldots$ & .28 & .30 & .20 & .25 & .07 & .03 & .01 & .03 & .05 & .16 & .20 & .05 \\
\hline $2 \ldots .$. & .08 & .03 & $\ldots \ldots$ & .01 & .19 & 1.14 & .03 & .25 & .22 & - & - & - \\
\hline $\begin{array}{l}3 \ldots \ldots \\
i_{5} \ldots \ldots\end{array}$ & $\begin{array}{ll}\cdots \cdots \\
\cdots \ldots\end{array}$ & $\cdots \cdots$ & & & $\ldots \ldots$ & $\ldots \ldots$ & $\cdots$ & $\cdots$ & * & * & * & * \\
\hline
\end{tabular}

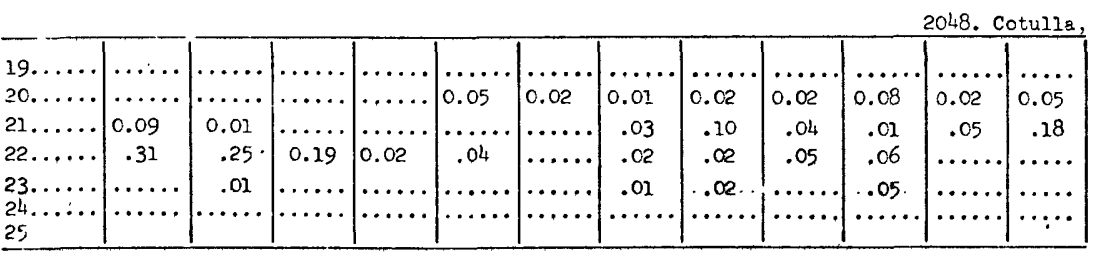

0569. Bay City Waterworks,

\begin{tabular}{|c|c|c|c|c|c|c|c|c|c|c|c|c|}
\hline & & & & & & & & & & & & \\
\hline & & & & & & L & $\cdots \cdots$ & $\cdots \cdots$ & & & & \\
\hline 20. & 0.02 & 0.03 & 0.07 & 0.08 & 0.07 & 0.09 & 0.10 & 0.19 & 0.05 & 0.02 & 0.01 & 0.02 \\
\hline $21 \ldots \ldots$ &. .03 & .03 & .03 & .22 & .38 & .68 & .24 & .15 & .18 & .59 & .06 & .03 \\
\hline $22 \ldots .$. & $\ldots \ldots$ & . . . . & $\ldots \ldots$ & $\ldots \ldots$ & $\ldots \ldots$ & $\ldots \ldots$ & $\ldots \ldots$ & $\ldots \ldots$ & $\ldots \ldots$ & ...... & ... & .... \\
\hline $23 . \ldots \ldots$ & $\cdots \cdots$ & $\ldots$. & $\ldots .$. & $\ldots$ & $\ldots \ldots$ & $\ldots \ldots$ & ..... & $\ldots$. & $\ldots \ldots$ & $\ldots \ldots$ & $\ldots \ldots$ & $\cdots \cdots$ \\
\hline${ }_{25}^{24} \ldots \ldots$ & ......... & $\cdots \cdots$ & $\ldots \ldots$ & $\ldots$ & $\ldots \ldots$ & $\ldots \ldots$ & $\ldots \ldots$ & ...... & $\ldots \ldots$ & $\ldots \ldots$ & $\ldots \ldots$ & $\ldots \ldots$ \\
\hline
\end{tabular}

See footnotes at end of table. 
Bureau weighing rain gages, storm of September 19-25, 1967-Continued

\begin{tabular}{|c|c|c|c|c|c|c|c|c|c|c|c|c|c|}
\hline 1300 & 1400 & 1500 & 1600 & 1700 & 1800 & 1900 & 2000 & 2100 & 2200 & 2300 & 24,00 & $\left(\begin{array}{c}\text { Daily } \\
\text { total } \\
\text { (inches) }\end{array}\right.$ & $\begin{array}{c}\text { Storm } \\
\text { total } \\
\text { (inches) }\end{array}$ \\
\hline
\end{tabular}

Duva] County

\begin{tabular}{|c|c|c|c|c|c|c|c|c|c|c|c|c|c|}
\hline & 0.22 & 0.38 & 0.01 & $\cdots$ & ..... & $\ldots \ldots$ & $\cdots$ & $\cdots$ & $\ldots$ & & 0.03 & 0.64 & \\
\hline 0.01 & .09 & .25 & .25 & 0.50 & 0.49 & 0.61 & 0.55 & 0.30 & 0.09 & 0.06 & .09 & 5.16 & \\
\hline 10 & .47 & .18 & .82 & . $\mathrm{Cl}_{4}$ & .25 & .18 & .01 & $\ldots \ldots$ & $\ldots \ldots$ & $\ldots \ldots$ & .01 & 2.77 & \\
\hline .30 & .19 & .37 & .18 & .09 &.$c 9$ & .01 & .01 & .01 & .15 & .10 & $\ldots$ & 4.10 & \\
\hline$\cdots \cdots$ & .05 & ..... & $\cdots \cdots$ & $\cdots \cdots$ & ..... & $\cdots \cdots$ & $\cdots \cdots$ & & ... & $\cdots$ & & 1.78 & \\
\hline.$n_{1}$ & .01 & $\ldots \ldots$ & $\ldots$ & - & $\ldots$. & $\ldots$. & $\ldots$ & $\ldots$ & & & & .45 & 31 \\
\hline
\end{tabular}

Gorl:"iles County

\begin{tabular}{|c|r|r|r|r|r|r|r|r|r|r|r|r|r|r}
\hline$\ldots \ldots$ & 0.17 & 0.05 & 0.03 & $\ldots \ldots$ & 0.01 & 0.15 & 0.34 & 0.08 & 0.02 & $\ldots \ldots$ & $\ldots \ldots$ & 0.85 & $\ldots \ldots \ldots$ \\
0.13 & .05 & .08 & .12 & 0.12 & .14 & .16 & .05 & $\ldots \ldots$ & .01 & 0.09 & 0.15 & 3.64 & $\ldots \ldots \ldots$ \\
.01 & .01 & .01 & .08 & 1.61 & .40 & .09 & .10 & .07 & .05 & .07 & .03 & 5.85 & $\ldots \ldots \ldots$ \\
$\ldots \ldots$ & $\ldots \ldots$ & $\ldots \ldots$ & $\ldots \ldots$ & $\ldots \ldots$ & $\ldots \ldots$ & $\ldots \ldots$ & $\ldots \ldots$ & $\ldots \ldots$ & $\ldots \ldots$ & $\ldots \ldots$ & $\ldots \ldots$ & .03 & $\ldots \ldots \ldots$ \\
$\ldots \ldots$ & $\ldots \ldots$ & $\ldots \ldots$ & $\ldots \ldots$ & $\ldots \ldots$ & $\ldots \ldots$ & $\ldots \ldots$ & $\ldots \ldots$ & $\ldots \ldots$ & $\ldots \ldots$ & $\ldots \ldots$ & $\ldots \ldots$ & $\ldots \ldots \ldots$ & $\ldots \ldots \ldots$ \\
$\ldots \ldots$ & $\ldots \ldots$ & $\ldots \ldots$ & $\ldots \ldots$ & $\ldots \ldots$ & $\ldots \ldots$ & $\ldots \ldots$ & $\ldots \ldots$ & $\ldots \ldots$ & $\ldots \ldots$ & $\ldots \ldots$ & $\ldots \ldots$ & $\ldots \ldots \ldots$ & $\ldots \ldots \ldots$ \\
\hline
\end{tabular}

Hidti] co Courity

\begin{tabular}{|c|r|r|r|r|r|r|r|r|r|r|r|r|r}
\hline 0.1 & 0.03 & 0.05 & 0.01 & 0.05 & 0.03 & 0.01 & $\ldots$. & $\ldots \ldots$ & 0.16 & 0.14 & 0.30 & 1.65 & $\ldots \ldots \ldots$ \\
.04 & .08 & .02 & .04 & $\ldots \ldots$ & .05 & .15 & 0.21 & 0.19 & .09 & .51 & .55 & 3.75 & $\ldots \ldots \ldots$ \\
1.0. & .05 & .05 & .03 & .01 & .05 & .01 & .02 & .01 & .01 & $\ldots \ldots$ & $\ldots \ldots$ & 5.55 & $\ldots \ldots \ldots$ \\
.01 & .05 & $\ldots \ldots$ & .24 & .05 & .02 & $\ldots \ldots$ & $\ldots \ldots$ & .01 & .01 & .01 & .01 & .50 & $\ldots \ldots \ldots$ \\
$\ldots \ldots$ & $\ldots \ldots$ & .01 & .01 & $\ldots \ldots$ & $\ldots \ldots$ & $\ldots \ldots$ & $\ldots \ldots$ & $\ldots \ldots$ & $\ldots \ldots$ & $\ldots \ldots$ & $\ldots \ldots$ & .17 & $\ldots \ldots \ldots$ \\
.33 & .01 & $\ldots \ldots$ & $\ldots \ldots$ & $\ldots \ldots$ & $\ldots \ldots$ & $\ldots \ldots$ & $\ldots \ldots$ & $\ldots \ldots$ & $\ldots \ldots$ & $\ldots \ldots$ & $\ldots \ldots$ & .34 & $\ldots \ldots \ldots$ \\
\hline
\end{tabular}

Kenu?y Courty

\begin{tabular}{|c|c|c|c|c|c|c|c|c|c|c|c|c|c}
\hline $0.7 !$ & 0.08 & 0.01 & 0.05 & 0.05 & 0.01 & 0.02 & $\ldots$. & 0.01 & $\ldots \ldots$ & 0.01 & 0.02 & 1.09 & $\ldots \ldots \ldots$ \\
.63 & .12 & .34 & .32 & .33 & .56 & .89 & 0.41 & .50 & 0.46 & .21 & .26 & 6.84 & $\ldots \ldots \ldots$ \\
.18 & .03 & .03 & .02 & .10 & .04 & .05 & .01 & .01 & .05 & .02 & .15 & 2.33 & $\ldots \ldots \ldots$ \\
$\ldots$ & - & - & - & - & $\ldots \ldots$ & $\ldots \ldots$ & $\ldots \ldots$ & $\ldots \ldots$ & $\ldots \ldots$ & $\ldots \ldots$ & $\ldots \ldots$ & 1.95 & $\ldots \ldots \ldots$ \\
$\ldots$ & $\ldots$. & $\ldots \ldots$ & $\ldots \ldots$ & $\ldots \ldots$ & $\ldots \ldots$ & $\ldots \ldots$ & $\ldots \ldots$ & $\ldots \ldots$ & $\ldots \ldots$ & $\ldots \ldots$ & $\ldots \ldots$ & $\ldots \ldots \ldots$ & $\ldots \ldots \ldots$ \\
$*$ & .76 & $\ldots \ldots$ & $\ldots \ldots$ & $\ldots \ldots$ & $\ldots \ldots$ & $\ldots \ldots$ & $\ldots \ldots$ & $\ldots \ldots$ & $\ldots \ldots$ & $\ldots \ldots$ & $\ldots \ldots$ & .76 & $\ldots \ldots \ldots$ \\
\hline
\end{tabular}

La : ille Courty

\begin{tabular}{|c|c|c|c|c|c|c|c|c|c|c|c|c|c|}
\hline & $\cdots$ & $\ldots \ldots$ & 0.25 & 0.02 & $\ldots \ldots$ & $\cdots$ & $\ldots \ldots$ & $\ldots \ldots$ & . & & & 0.27 & \\
\hline $0.0:$ & 0.20 & 0.33 & .30 & .13 & 0.12 & . & 0.01 & 0.03 & C.Ol & & 0.05 & 1.52 & \\
\hline .1 & .21 & .24 & .04 & .08 & .02 & 0.06 & .04 & .05 & .15 & 0.55 & .20 & 2.17 & \\
\hline .1. & .05 & $\ldots \ldots$ & .01 & $\cdots$ & .01 & $\ldots$. & .01 & $\ldots$. & $\ldots$. & $\ldots$ & .02 & 1.22 & \\
\hline$\cdots \cdots$ & $\cdots \ldots$ & $\ldots \ldots$ & $\cdots \cdots$ & $\cdots \cdots$ & $\ldots \ldots$ & $\because$ & & & & $\cdot$ & • & .09 & \\
\hline$\cdots \cdots$ & $\cdots$ & & & ..... & 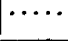 & & & & & & .... & & $z$ \\
\hline
\end{tabular}

Metneorda Cour.ty

\begin{tabular}{|c|c|c|c|c|c|c|c|c|c|c|c|c|c|}
\hline$\cdots$ & $\cdots$ & $\ldots \ldots$ & $\ldots \ldots$ & 0.02 & 0.29 & 0.26 & 0.03 & 0.05 & 0.03 & 0.06 & 0.02 & 0.76 & \\
\hline 0.03 & .01 & 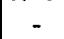 & - & 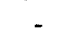 & 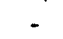 & - & 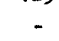 & - & $\because$ & - & - & 2.56 & $\cdots$ \\
\hline$\cdots \cdots$ & $\ldots \ldots$ & $\ldots$ & $\cdots$ & .... & $\ldots \ldots$ & $\ldots$ & ..... & $\cdots \cdots$ & $\ldots \ldots$ & $\ldots$. & $\cdots$ & n....... & •. \\
\hline$\cdots \cdots$ & $\ldots$. & $\cdots \ldots$ & $\ldots \ldots$ & .10 & ..... & $\ldots \ldots$ & ..... & $\cdots$ & $\cdots$ & $\ldots \ldots$ & $\cdots \cdots$ & .10 & $\cdots \cdots$ \\
\hline . & $\cdots$ & ... & $\ldots \ldots$ & .... & $\ldots \ldots$ & $\ldots \ldots$ & $\cdots:$ & $\cdots$ & $\cdots$ & $\ldots \ldots$ & $\cdots$ & & 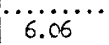 \\
\hline
\end{tabular}


$\mathrm{T}_{\mathrm{ABLE}}$ 5.-Hourly precipitation, in inches, at U.S. Weather

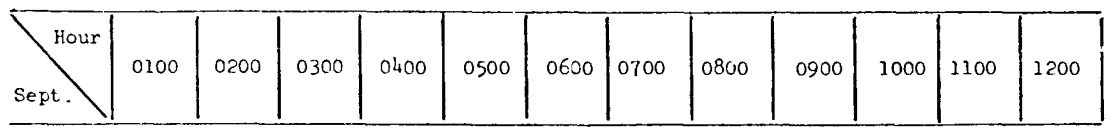

2015. Corpus Christi WB Airport,

\begin{tabular}{|c|c|c|c|c|c|c|c|c|c|c|c|c|}
\hline $190^{\circ}$ & & & & & ריר? & $a^{\prime}$ & נית & & & & & \\
\hline 20. & $\begin{array}{l}\cdots \\
0.03\end{array}$ & 0.03 & 0.03 & 0.01 & $\begin{array}{r}0.27 \\
.07\end{array}$ & $\int_{23}^{c .0 .4}$ & 0.01 & 0.28 & 0.11 & 0.149 & 0.42 & 0.12 \\
\hline 21. & $\ldots \ldots$ & .01 & $\ldots \ldots$ & .37 & .03 & , . . & $\cdots$ & $\cdots$ & .07 & .06 & 1.37 & 1.23 \\
\hline $22 .$. & $\ldots \ldots$ & $\ldots \ldots$ & .. & $\ldots \ldots$ & .38 & 1.13 & .41 & .33 & .27 & .10 & .08 & .01 \\
\hline $\begin{array}{l}23 \ldots \ldots \\
24 \ldots \ldots \\
25\end{array}$ & $\begin{array}{l}\cdots \cdots \\
\cdots \cdots\end{array}$ & $\cdots \ldots$ & & . & $\ldots$ & .29 & . & & . & & $\cdots$ & $0^{\circ}$ \\
\hline
\end{tabular}

\begin{tabular}{|c|c|c|c|c|c|c|c|c|c|c|c|c|}
\hline \multicolumn{13}{|c|}{ 9364. Victoria WB Airport } \\
\hline $19 \ldots \ldots$ & $\ldots \ldots$ & $\cdots$ & & $\cdots \cdots$ & $\cdots$ & & & & & & & \\
\hline $20 \ldots \ldots$ & 0.03 & 0.07 & $c .20$ & 0.38 & c.17 & c.13 & 0.50 & 0.90 & 0.48 & 0.16 & 0.80 & 0.01 \\
\hline $21 \ldots \ldots$ & .26 & .70 & .95 & .54 & .10 & .10 & .30 & 1.31 & .25 & .32 & .10 & .03 \\
\hline $22 \ldots \ldots$ & $\ldots \ldots$ & $\ldots \ldots$ & $\cdots \cdots$ & $\ldots \ldots$ & $\ldots$. & $\ldots \ldots$ & $\ldots \ldots$ & $\ldots \ldots$ & $\cdots$ & $\ldots \ldots$ & ..... & $\cdots$ \\
\hline $23 \ldots \ldots$ & $\ldots \ldots$ & $\ldots \ldots$ & $\ldots \ldots$ & $\ldots \ldots$ & $\ldots \ldots$ & $\ldots \ldots$ & $\ldots \ldots$ & $\ldots \ldots$ & $\ldots \ldots$ & $\ldots \ldots$ & $\cdots \cdots$ & $\cdots$ \\
\hline $\begin{array}{l}24 \ldots \ldots \\
25\end{array}$ & $\ldots \ldots$ & $\ldots \ldots$ & $\ldots \ldots$ & $\ldots \ldots$ & $\ldots \ldots$ & $\ldots \ldots$ & & & & & & \\
\hline
\end{tabular}

5c60. Lerado No. 2,

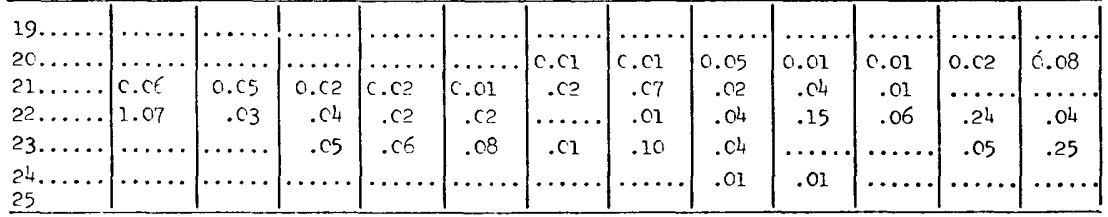

9976. Zopata,

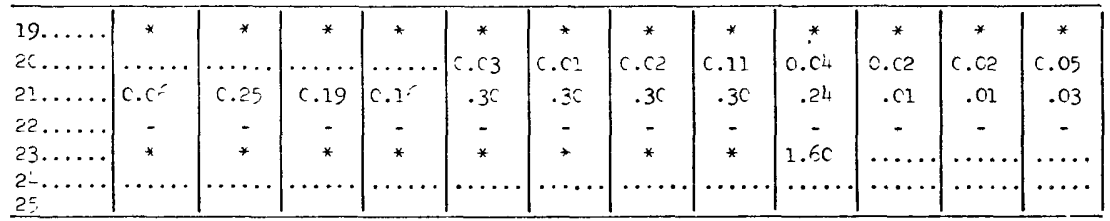

- No record.

- Arount included in a following measurement, time distribution unknown.

volume of water carried by the Rio Grande and its floodways was runoff from the Rio Alamo and Rio San Juan watersheds in Nuevo Leon and Tamaulipas, Mexico. Soil moisture and other factors that influence the runoff rate ranged from extremely favorable for a high percentage of runoff in the Rio Alamo and Rio San Juan watersheds to near or below normal in the Lavaca River basin.

\section{LAVACA RIVER BASIN}

The headwaters of the Lavaca River are on the east edge of the heavy precipitation area. In this basin, rainfall ranged from 5 to 15 inches, with the lesser amount occurring in the headwaters area. The weather station at Edna in Jackson County reported a 2-day total rainfall of 11.60 inches on September 20 and 21. Rainfall of this amount has a recurrence interval of greater than 25 years. 
Bureau weighing rain gages, storm of September 19-25, 1967-Continued

\begin{tabular}{|c|c|c|c|c|c|c|c|c|c|c|c|c|c|}
\hline 1300 & 14,00 & 1500 & 1600 & 1700 & 1800 & 1900 & 2000 & 2100 & 2200 & 2300 & 2400 & $\begin{array}{c}\text { Daily } \\
\text { total } \\
\text { (inches) }\end{array}$ & $\begin{array}{c}\text { Storm } \\
\text { total } \\
\text { (inches) }\end{array}$ \\
\hline
\end{tabular}

in eees Contity

\begin{tabular}{|c|c|c|c|c|c|c|c|c|c|c|c|c|c|}
\hline & 0.35 & $(\cdot .5$ & 0.15 & 0.03 & $\cdot$ & . & & 0.03 & & $\ldots \ldots$ & & 0.90 & \\
\hline$n .1$, & $\ldots$ & $\ldots \ldots$ & $\ldots \ldots$ & .09 & 0.14 & 0.10 & 0.25 & .12 & $0.1 t_{+}$ & 0.05 & 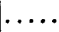 & 3.48 & \\
\hline .1 .4 & .25 & . ́́8 & .76 & .05 & .58 & $\ldots \ldots$ & .01 & .72 & .01 & .04 & $\cdots$ & 6.38 & \\
\hline . & .01 & $\ldots \ldots$ & $\ldots \ldots$ & $\ldots \ldots$ & $\ldots \ldots$ & $\ldots \ldots$ & $\ldots \ldots$ & $\ldots \ldots$ & $\ldots \ldots$ & $\ldots \ldots$ & & 3.02 & \\
\hline & $\cdots$ & $\ldots \ldots$ & $\ldots$ & $\cdots$ & $\ldots$ & $\cdots \cdot$ & & $\ldots$ & $\cdots \cdots$ & …. & $\cdots$ & .29 & $\cdots \ldots \ldots$ \\
\hline & & & $\cdots$ & $\cdots \cdots$ & .. & & $\ldots$ & & $\ldots \ldots$ & .. & . . & $\cdots \cdots$ & $\dddot{14}_{14.07^{\prime}}$ \\
\hline
\end{tabular}

vicetnria Cour.ty

\begin{tabular}{|c|c|c|c|c|c|c|c|c|c|c|c|c|c|}
\hline 0.17 & 0.12 & 0.05 & 0.01 & 0.01 & 0.24 & 0.25 & 0.04 & $\cdots$ & & 0.10 & 0.07 & 1.07 & \\
\hline .05 & .12 & .06 & .08 & 1.63 & .43 & .01 & .01 & 0.01 & 0.05 & .15 & .20 & 6.63 & \\
\hline$\ldots \ldots$ & .05 &.$c 8$ & .04 & .05 & $\ldots \ldots$ & .07 & .... & .01 & $\ldots$ & .01 & .... & 5.27 & \\
\hline. & $\ldots$ & $\ldots$ & $\cdots \cdots$ & $\cdots \cdot$ & $\ldots$. & $\cdots \cdots$ & $\cdots$ & $\ldots \ldots$ & . & $\cdots \cdots$ & .... & $\ldots \ldots \ldots$ & $\cdots$ \\
\hline$\cdots \cdots$ & $\cdots$ & $\cdots$ & $\cdots \cdots$ & $\cdots$ & $\ldots \ldots$ & $\ldots \ldots$ & $\cdots \cdots$ & $\cdots \cdots$ & $\cdots$ & $\cdots \cdots$ & $\ldots \ldots$ & ......... & $\cdots$ \\
\hline$\ldots$ & .. & $\ldots$ & $\ldots \ldots$ & $\cdots \cdots$ & $\ldots \ldots$ & $\ldots$ & $\ldots$ & $\ldots \ldots$ & $\ldots \ldots$ & $\ldots$ & .... & $\cdots$ & 12.97 \\
\hline
\end{tabular}

Sol county

\begin{tabular}{|c|c|c|c|c|c|c|c|c|c|c|c|c|c|}
\hline & & & 0.49 & 0.18 & 0.02 & $\cdots$ & & & & & & 0.69 & \\
\hline C.c4 & $0.1 \mathrm{C}$ & c.c5 & .10 & .05 & .01 & $\ldots$ & 0.19 & C.16 & 0.10 & 0.08 & 0.04 & 1.11 & \\
\hline$\ldots \ldots$ & $\ldots \ldots$ & $\ldots \ldots$ & .03 & .01 & .11 & 0.21 & .49 & .25 & .24 & .15 & .22 & 2.03 & . \\
\hline .20 &. $\mathrm{Cl}$ &.$C 1$ & $\ldots \ldots$ & $\ldots \ldots$ & .05 & .19 & .11 & .05 & .... & $: 01$ & . . . & 2.35 & . \\
\hline$\ldots$ & .05 & ... & $\ldots$. & $\ldots$. & .02 & $\ldots \ldots$ & $\ldots$ & $\cdots$ & . & $\cdots$ & $\ldots$ & .71 & \\
\hline$\ldots \ldots$ & $\ldots$. & .07 & .02 & .01 & $\ldots$. & $\ldots$ & $\ldots$ & $\ldots$. & $\ldots$ & $\ldots$. & $\ldots \ldots$ & .12 & ${ } \ldots_{7.01}{ }{ }$ \\
\hline
\end{tabular}

7ispasea County

\begin{tabular}{|c|c|c|c|c|c|c|c|c|c|c|c|c|c|}
\hline$*$ & * & + & * & 0.47 & c.cl. & 0.06 & 0.09 & & & & & 0.65 & \\
\hline$\cdots$ & $\ldots$. & ...5́ & C.CE & . cs &.$C ?$ & .02 & .58 & $c . \equiv 7$ & $c .27$ & C.11 & 0.05 & $1.9=$ & \\
\hline ויי. & .65 & .05 & .09 & .03 & - & - & - & - & - & - & . & 2.48 & \\
\hline - & - & - & - & - & - & - & - & - & - & - & - & - & \\
\hline$\cdots$ & ..... & $\ldots \ldots$ & $\cdots$ & $\cdots$ & $\cdots$ & $\cdots \cdot$ & $\cdots \cdots$ & $\cdots$ & $\cdots$ & $\cdots$ & $\cdots$ & 1.60 & \\
\hline . lt? & .15 & $\ldots$ & $\ldots$ & $\ldots$ & $\ldots$ & ... & .. & $\ldots$ & $\ldots$ & . & ..... & .57 & 7.26 \\
\hline
\end{tabular}

Runoff was substantial, although less than would be expected from the rainfall frequency. This result, based on a daily discharge of $3.6 \mathrm{cfs}$ (cubic feet per second) on Septernber 19 at the streamflow station near Edna (site 1), can be partly attributed to fairly low soil-moisture content in the basin. On the $23 \mathrm{~d}$, a peak discharge of $22,500 \mathrm{cfs}$ at a gage height of 26.37 feet was recorded. Flood stage at Edna is 21 feet. A flood of this magnitude has a recurrence interval of about 4 years.

The Navidad River, a tributary of the Lavaca River, had a peak discharge of 26,600 cfs from 1,116 square miles with a stage of 31.91 feet at the streamflow station near Ganado (site 2). Flood stage at Ganado is 21 feet. A flood of this magnitude has a recurrence interval of about 4 years.

Flooding did occur in the Lavaca River basin, but flood damage was minor, and the flood did not approach the magnitude of previous floods. 


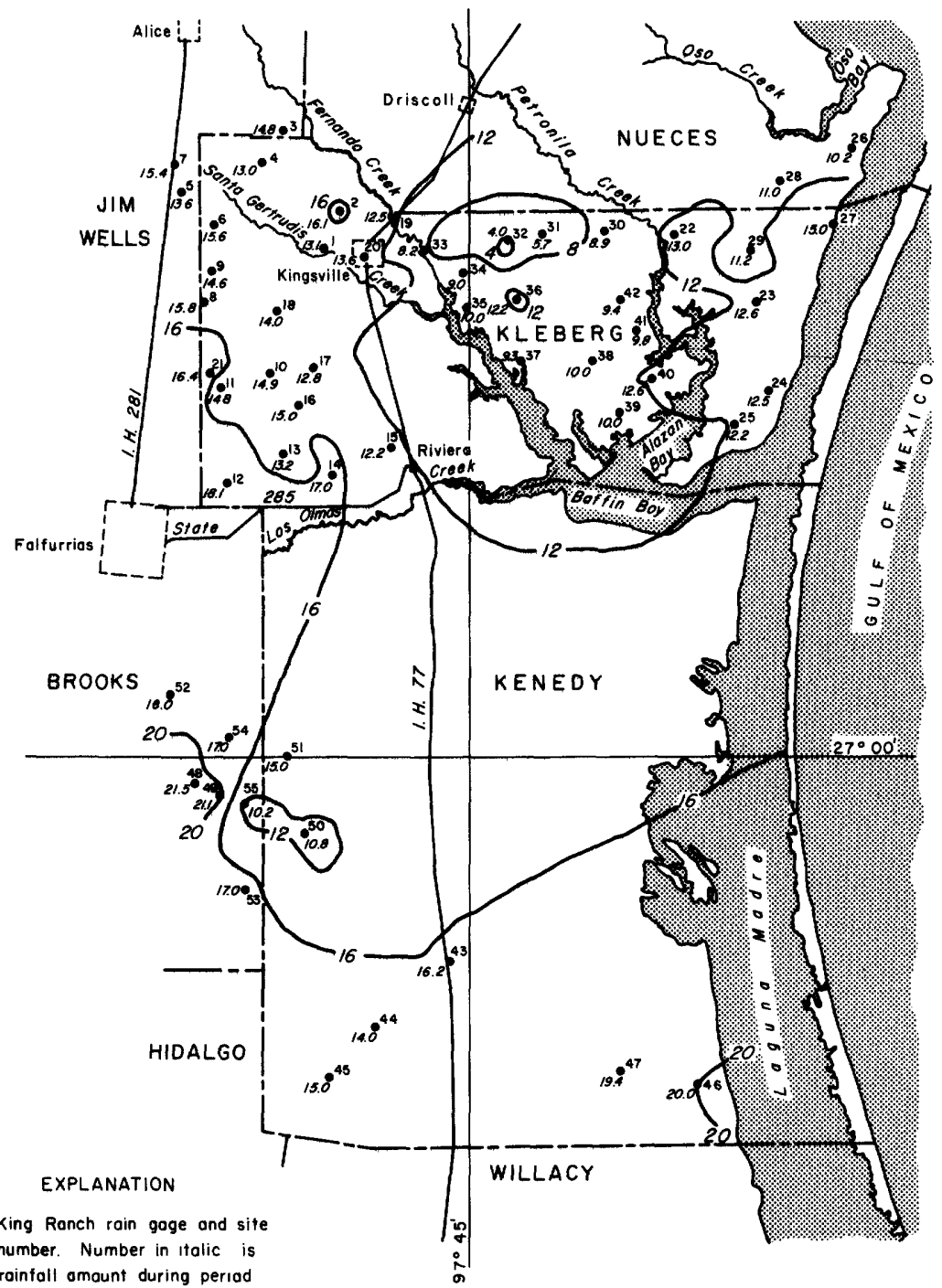

September 18-25, 1967

-16 isohyet, line of appraxımate equal rainfoll

FigURF 5.--Isohyetal and location of rain-gage network on the King Ranch.

\section{GUADALUPE RIVER BASIN}

The Guadalupe River basin received rainfall ranging from 1.75 inches at Kerrville in Kerr County, near the headwaters, to about 25 inches in the Coleto Creek area upstream from the gaging station at Schroeder in Goliad County. The greatest amount of rainfall recorded during the 


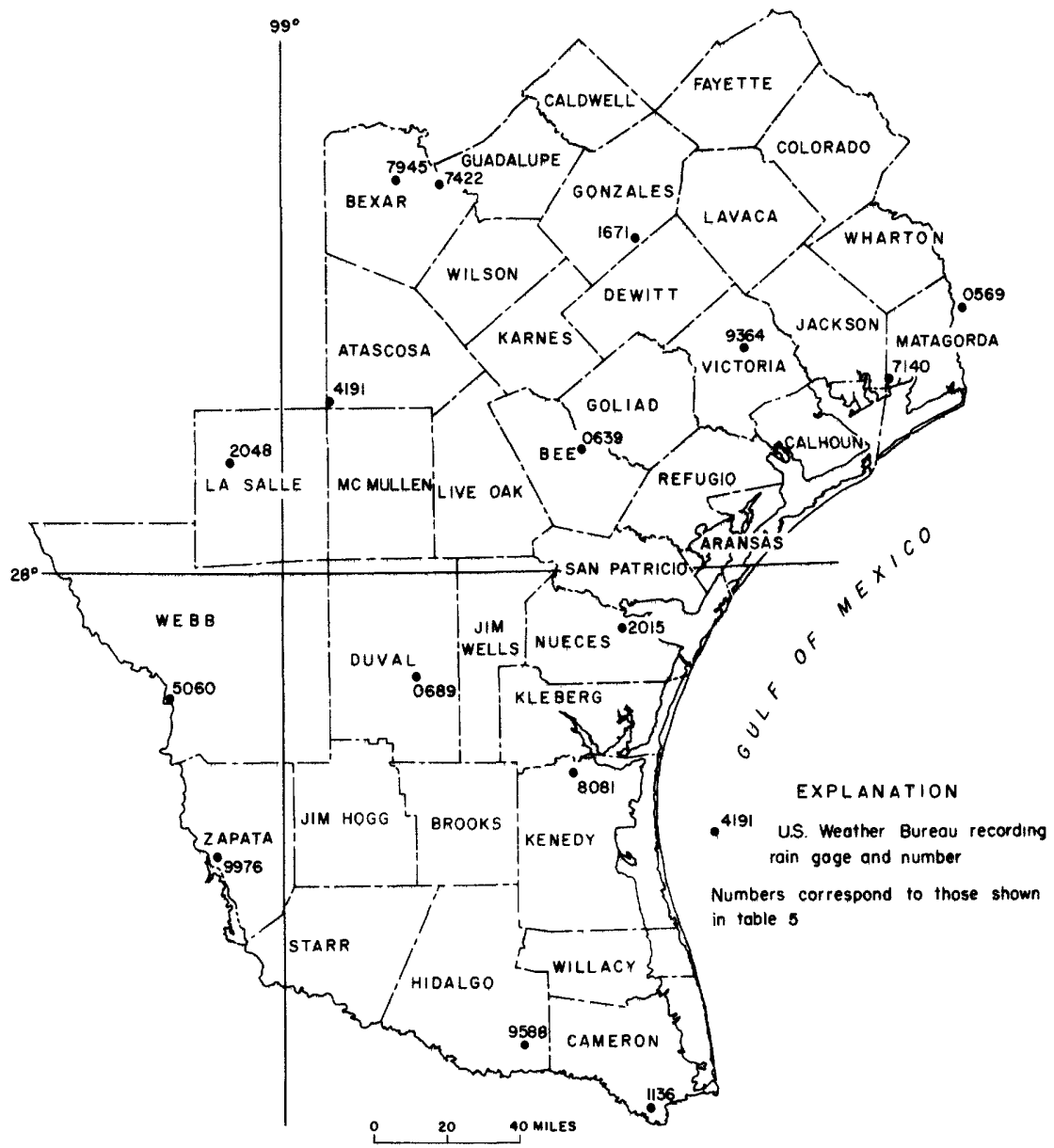

Figure 6.-Location of U.S. Weather Bureau recording rain gages.

storm period at a regular weather station was 22.28 inches at Yorktown in DeWitt County, although larger amounts were reported at supplemental sites in this basin. Yorktown received a 2-day total rainfall for September 21 and 22 of 20.11 inches. Cuero, also in DeWitt County, had a 2-day total rainfall of 18.35 inches for the same period. These amounts greatly exceed the 2-day, 100-year frequency.

In downstream order, the principal streams tributary to the Guadalupe River are the San Marcos River, Peach Creek, Sandies Creek, and Coleto Creek. Runoff in the basin above the mouth of the San Marcos River was light.

Peach Creek, in a 5- to 10-inch rainfall zone in Gonzales County, had a peak discharge of $10,200 \mathrm{cfs}$ at the streamflow station near Dilworth (site 5). A flood of this magnitude has a recurrence interval of 3 years, 
CORPUS CHRISTI, TEXAS

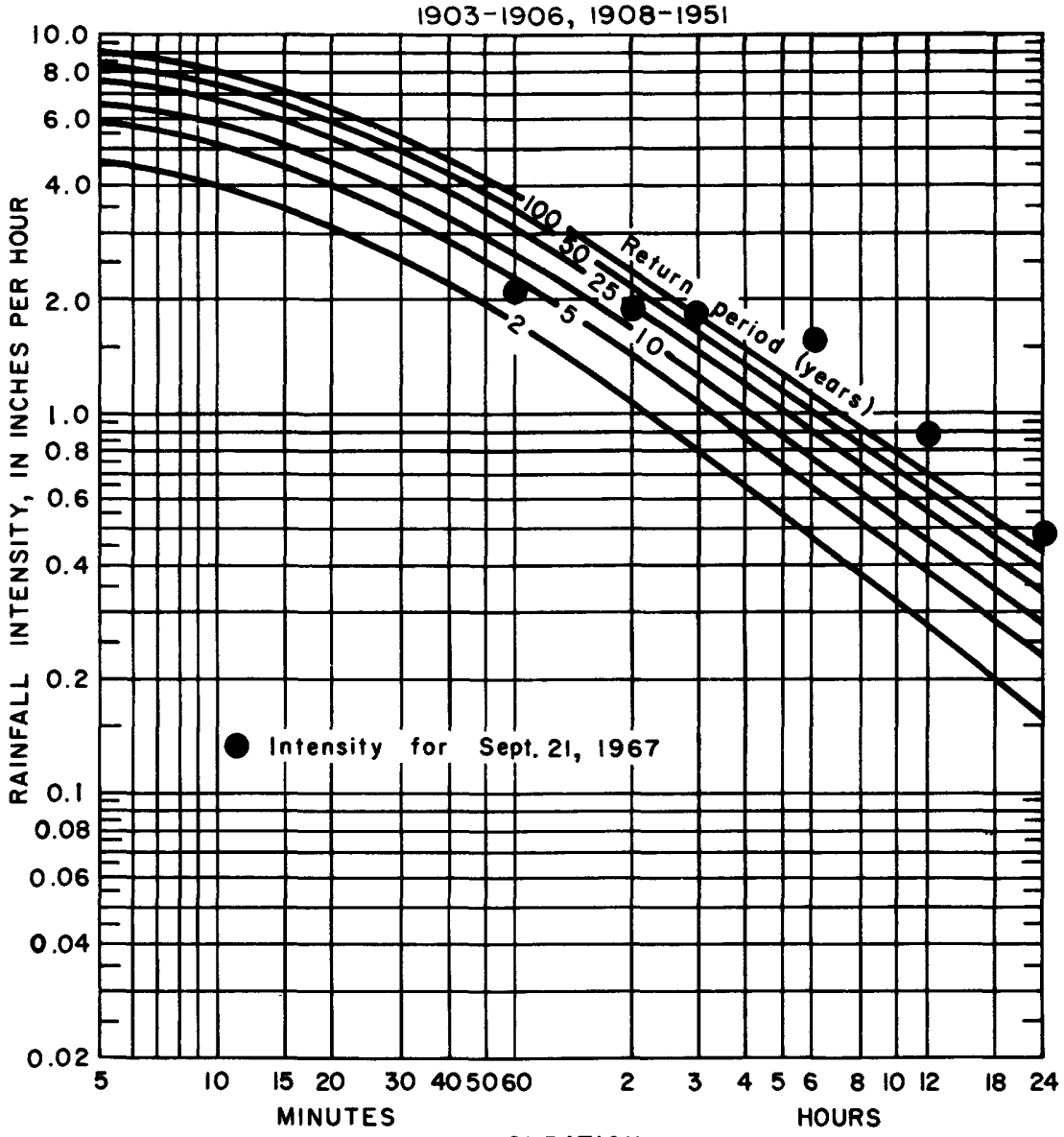

DURATION

From U.S. Weother Bureau Technical Paper No. 25

Figure 7.-Comparison of the rainfall intensity-duration-frequency curve for Corpus Christi and the maximum point rainfall intensity at U.S. Weather Bureau rain gage 0639 , Beeville $5 \mathrm{NE}$, September 21, 1967. Frequency analysis by method of extreme values, from Gumbel, 1954.

Sandies Creek watershed, in Guadalupe, Gonzales, Wilson, Karnes, and DeWitt Counties, received rainfall totals during the storm period ranging from less than 10 inches at the headwaters in Guadalupe County to more than 20 inches at the streamflow station near Westhoff in DeWitt County. At a site near Leesville, Sandies Creek (site 6) had a peak discharge of $3,920 \mathrm{cfs}$ from a drainage area of 47.4 square miles. This discharge has a recurrence interval of 3 years. At the streamflow 




DURATION

Figure 8.-Comparison of the rainfall intensity-duration-frequency curve for San Antonio and the maximum point rainfall intensity at U.S. Weather Bureau rain gage 0639, Beeville 5 NE, September 21, 1967. Frequency analysis by method of extreme values, from Gumbel, 1954.

station near Westhoff (site 7), the peak discharge was 79,700 cfs from a drainage area of 560 square miles. The recurrence interval for this discharge is 35 years.

Coleto Creek watershed, in DeWitt, Goliad, and Victoria Counties, received 20 to 25 inches of rainfall during the storm period. These were the greatest amounts reported in the Guadalupe River basin, and the 

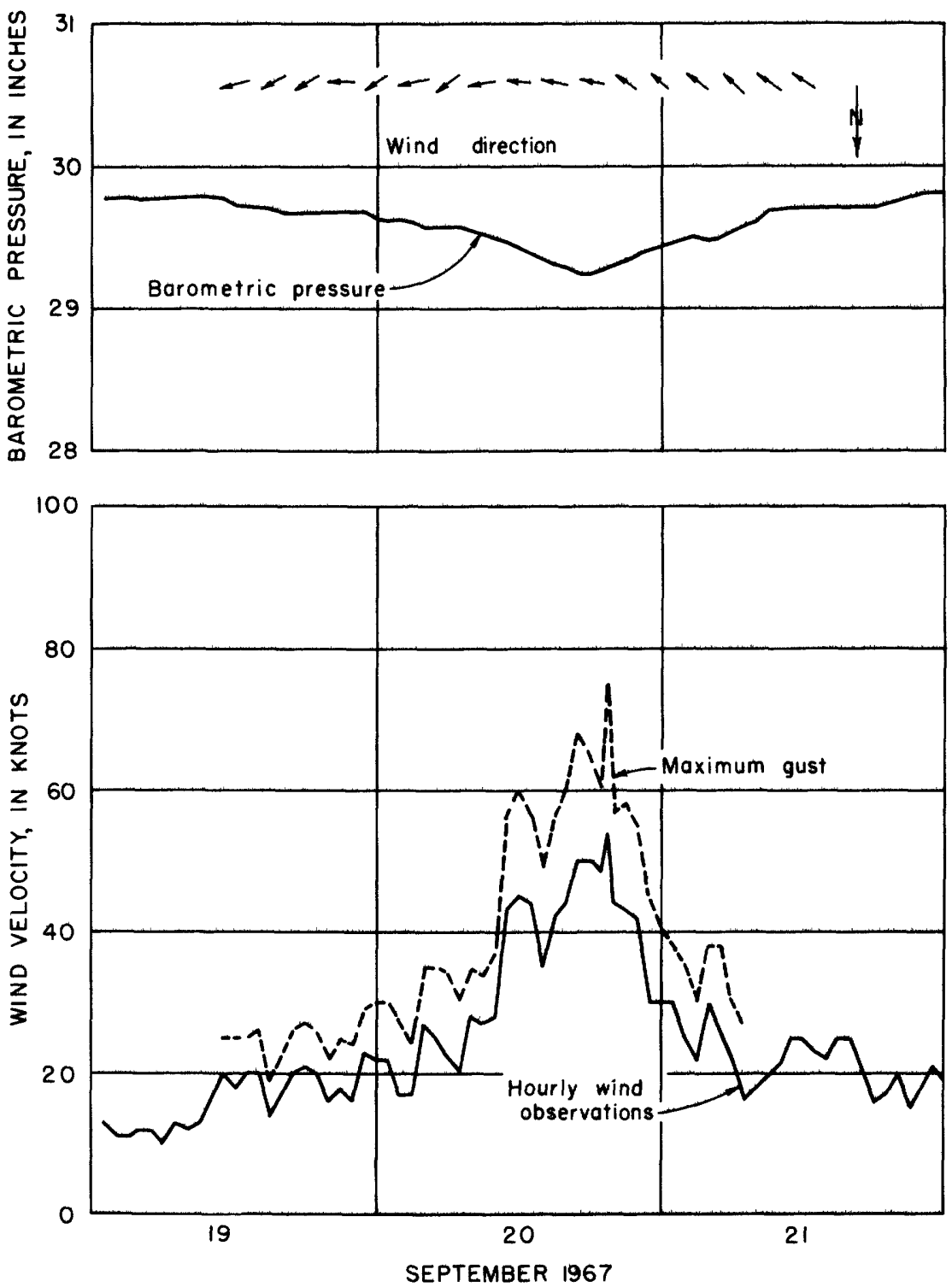

Figure 9.-Barometric pressure and wind velocity at the U.S. Weather Bureau station, Corpus Christi, Tex. Data furnished by U.S. Army Engineer District, Galveston, Tex.

consequent flooding on Coleto Creek was greater than any previously observed during the period of historical record dating back to 1872 . The peak discharge from the 365-square-mile drainage area above the streamflow station near Schroeder (site 12) was $122,000 \mathrm{cfs}$, which is 

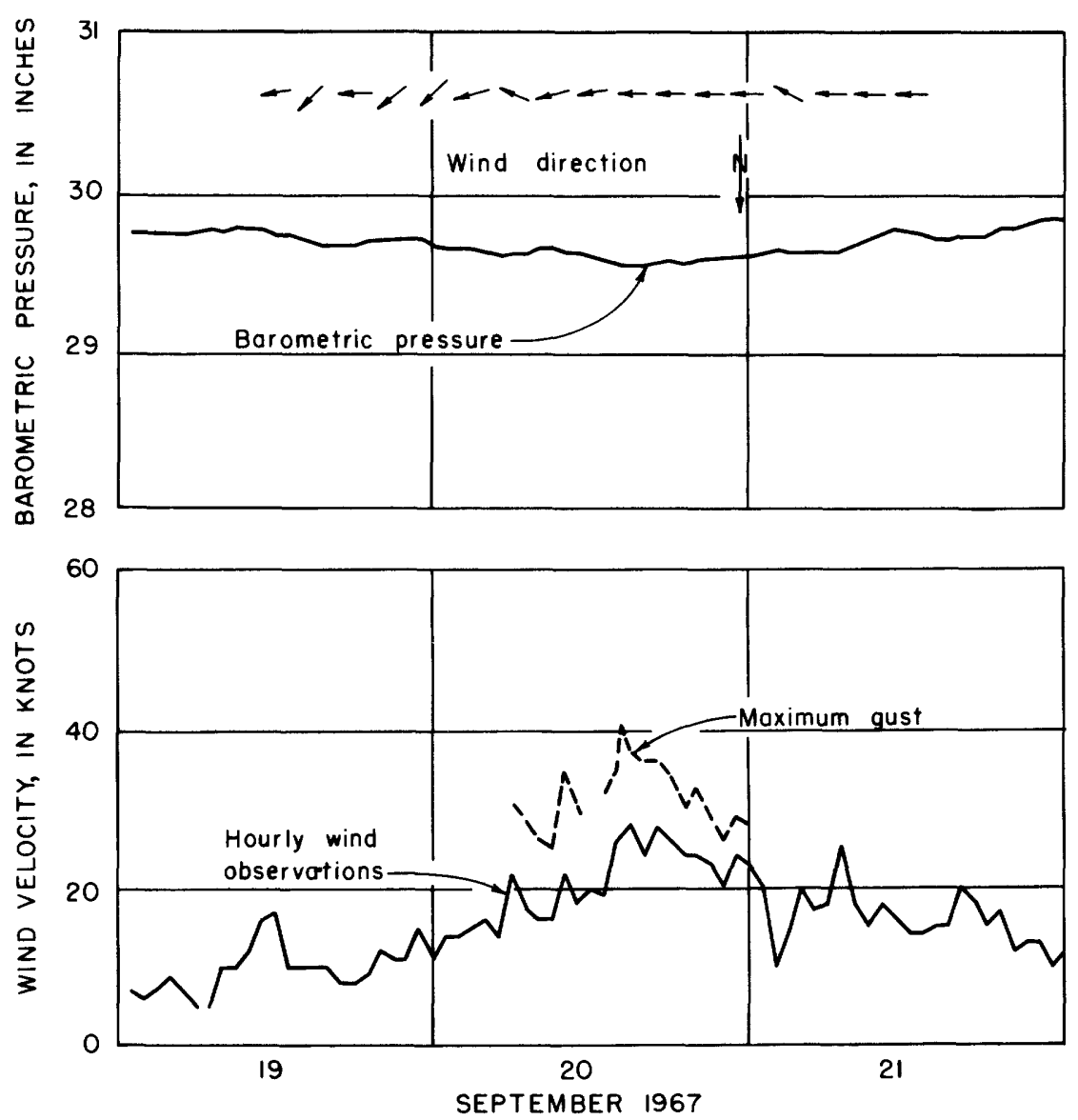

FIGURE 10.-Barometric pressure and wind velocity at the U.S. Weather Bureau station,

Victoria, Tex. Data furnished by U.S. Army Engineer District, Galveston, Tex.

1.73 times the magnitude of a 50-year flood. At the discontinued gagingstation site near Victoria (site 13) in Victoria County, the peak discharge from a drainage area of 514 square miles was $236,000 \mathrm{cfs}$, which is 2.74 times the magnitude of a 50 -year flood and is 2.65 times greater than the previously recorded maximum.

A peak discharge of $70,000 \mathrm{cfs}$ was recorded on the Guadalupe River on September 21 at the gaging station near Victoria (site 10). This runoff was generated primarily in the 284 -square-mile area between the Cuero station and the Victoria station. The upstream rise, which had a peak discharge of $61,500 \mathrm{cfs}$ at Cuero (site 8), did not reach the Victoria station until September 24. The peak discharge at Cuero and Victoria has a recurrence interval of 10 and 14 years, respectively.

Flooding also occurred on the many smaller streams in the basin. At 


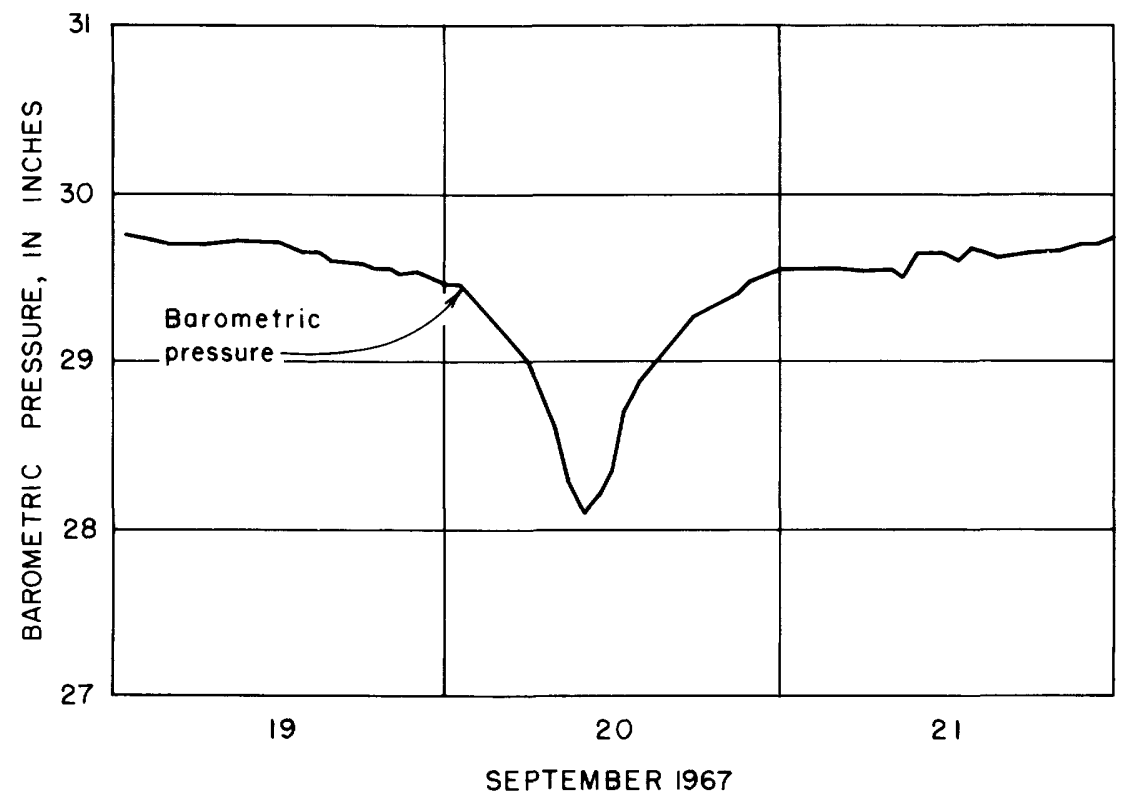

Figure 11.-Barometric pressure at Harlingen, Tex. Data by U.S. Army Engineer District, Galveston, Tex.

the present time, data from this area are not sufficient to define a frequency and magnitude relation for streams having a drainage area of less than 30 square miles, but peak stage and discharge data for four small streams are included in table 17.

Flood damage in the Guadalupe River basin was most severe along the lower reaches of Sandies Creek, downstream from Westhoff; along all of Coleto Creek; and along the main stem below Victoria, especially below the mouth of Coleto Creek. In these areas, inundation damaged railways, highways, and utilities.

\section{SAN ANTONIO RIVER BASIN}

In the San Antonio River basin, rainfall during the period ranged from 1.92 inches at Medina in Bandera County, near the headwaters of the Medina River, to 25 inches in the lower Cibolo Creek watershed near the Wilson-Karnes County line. The 2-day, 100-year frequency was exceeded at a number of weather stations. The greatest 2-day total (25.50 in.) at a regular weather station was recorded at the site of the streamflow station Cibolo Creek near Falls City (site 22) in Karnes County.

In downstream order, the principal streams tributary to the San Antonio River are Medina River, Calaveras Creek, Cibolo Creek, Ecleto Creek, and Escondido Creek. 

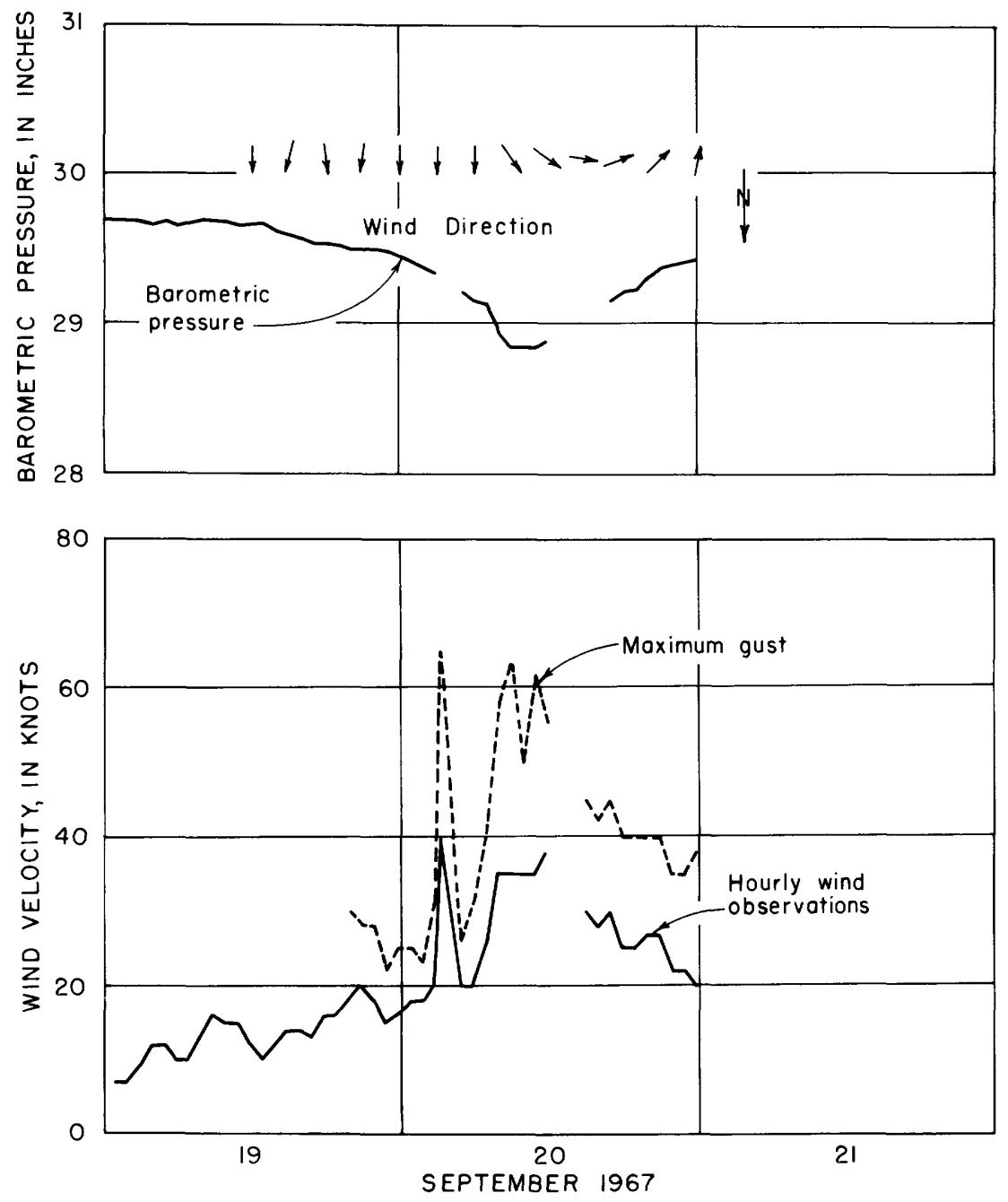

Figure 12.-Barometric pressure and wind velocity at McAllen, Tex., airport. Data furnished by U.S. Army Engineer District, Galveston, Tex.

Very little overbank flooding occurred in the San Antonio River basin above the mouth of the Medina River. However, local flooding did occur in the city of San Antonio, where at least two people were drowned at low-water crossings.

Runoff from the Medina River and Calaveras Creek watersheds was comparatively light. A peak discharge of $5,480 \mathrm{cfs}$ was recorded at Medina River near San Antonio (site 16) and 3,720 cfs was recorded at Calaveras Creek near Elmendorf (site 19) in Bexar County. Medio Creek, a tributary of the Medina River, had a peak discharge of 2,640 

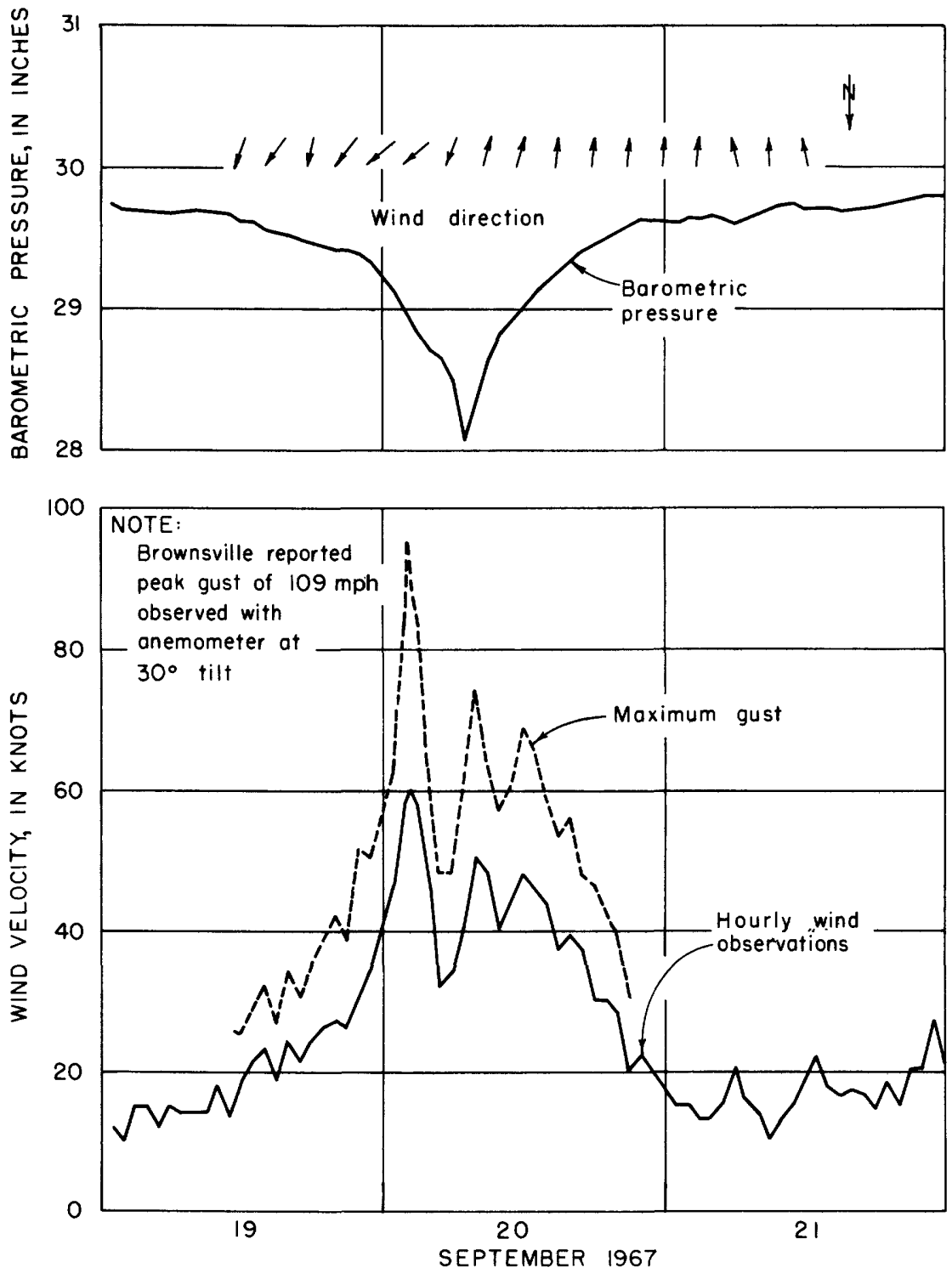

FiguRE 13.-Barometric pressure and wind velocity at Brownsville, Tex., airport. Data furnished by U.S. Army Engineer District Galveston, Tex.

cfs from a drainage area of 47.9 square miles at a miscellaneous site near Macdona (site 15) in western Bexar County.

Cibolo Creek, which drains parts of Kendall, Comal, Bexar, Guadalupe, Wilson, and Karnes Counties, had very light runoff up- 


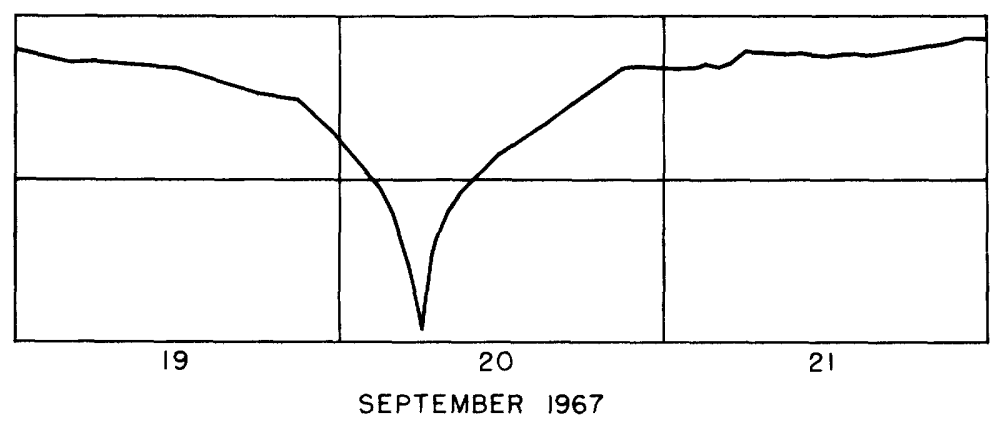

Figure 14.-Barometric pressure at Port Isabell Coast Guard Station, South Padre Island.

Data furnished by U.S. Weather Bureau.

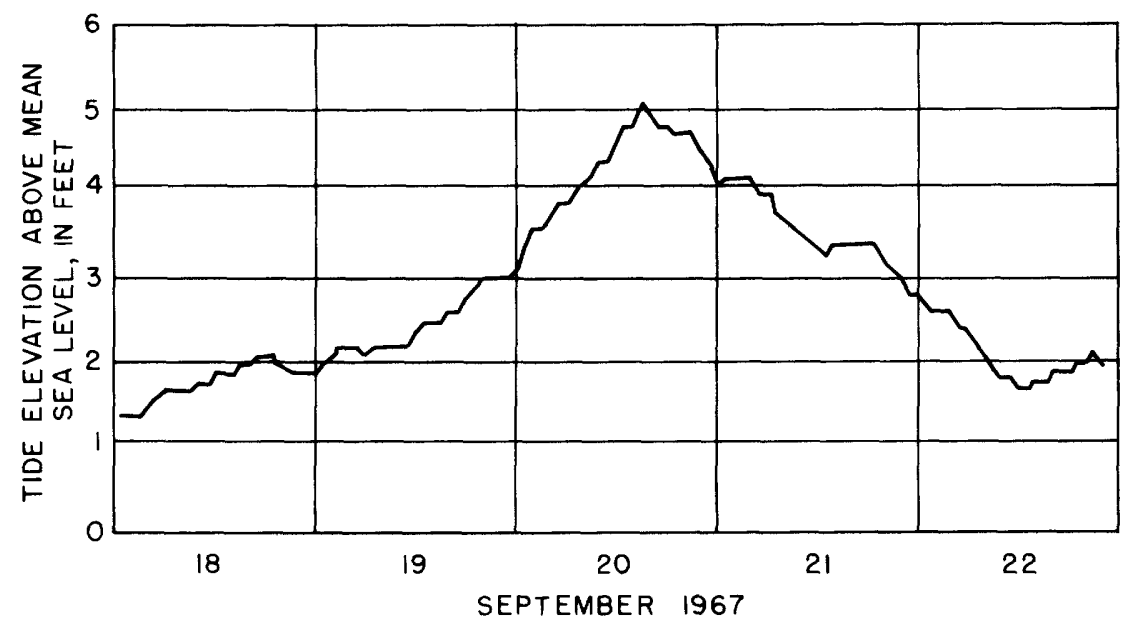

Figure 15.-Tide elevation at Matagorda Peninsula gage.

stream from the streamflow station at Selma (site 21) in Bexar County. During this storm, a peak discharge of only $89 \mathrm{cfs}$ was recorded, which was not the maximum for September. At the downstream station near Falls City (site 22) in Karnes County, a maximum discharge of 25,300 cfs was recorded from 827 square miles. The runoff in the lower part of the watershed, produced by rainfall that ranged from 10 to 25 inches, has a recurrence interval of 9 years.

Ecleto Creek watershed in Wilson and Karnes Counties had 10 to 20 inches of rain during the storm period. Runoff was very high in the 239 square-mile area above the streamflow station near Runge (site 23) in Karnes County, where a peak discharge of $58,400 \mathrm{cfs}$ was recorded. This discharge is 2.24 times the magnitude of a 50 -year flood. 


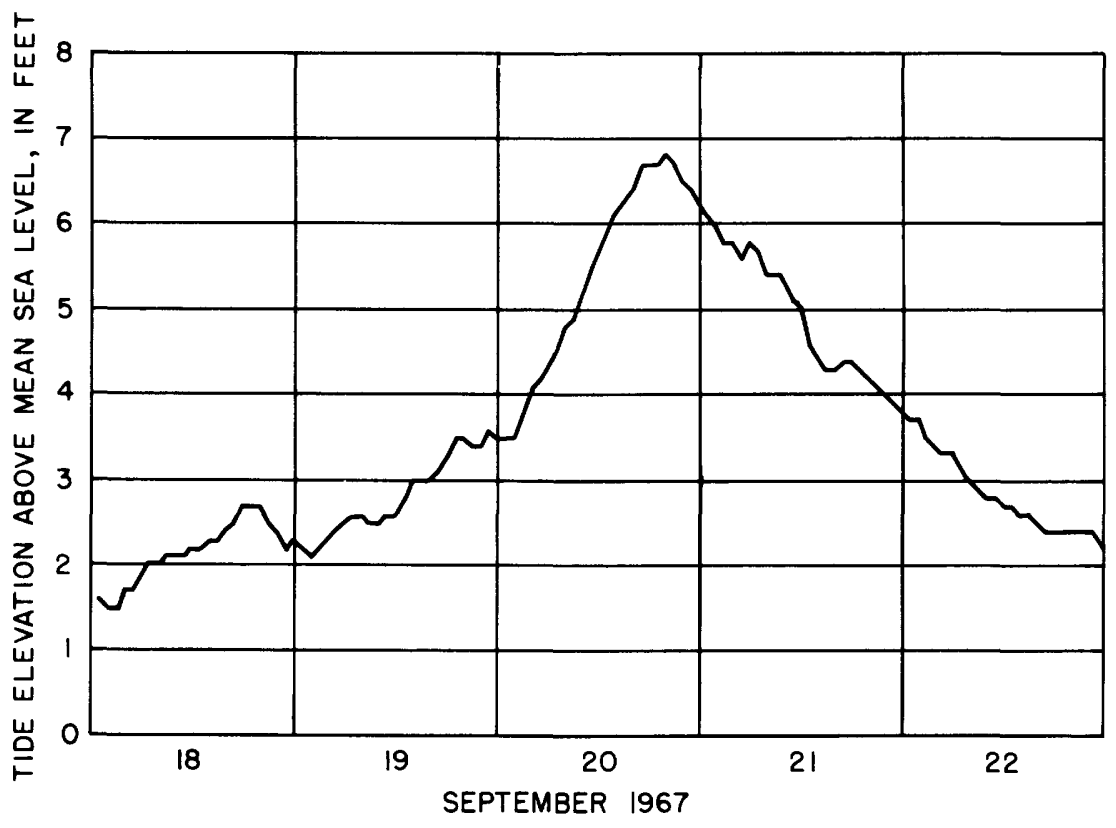

FigURE 16.-Tide elevation in Lavaca Bay at Interstate Highway 35 causeway.

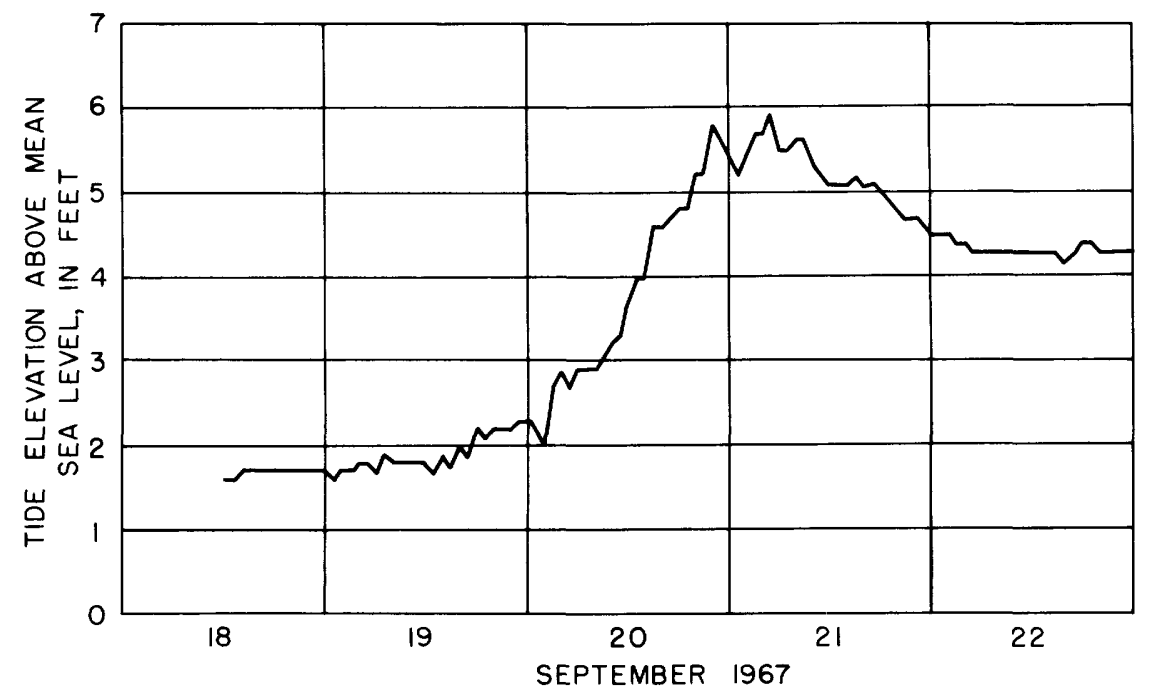

Figure 17.-Tide elevation in San Antonio Bay at Benderwald Point. 
ᄂu

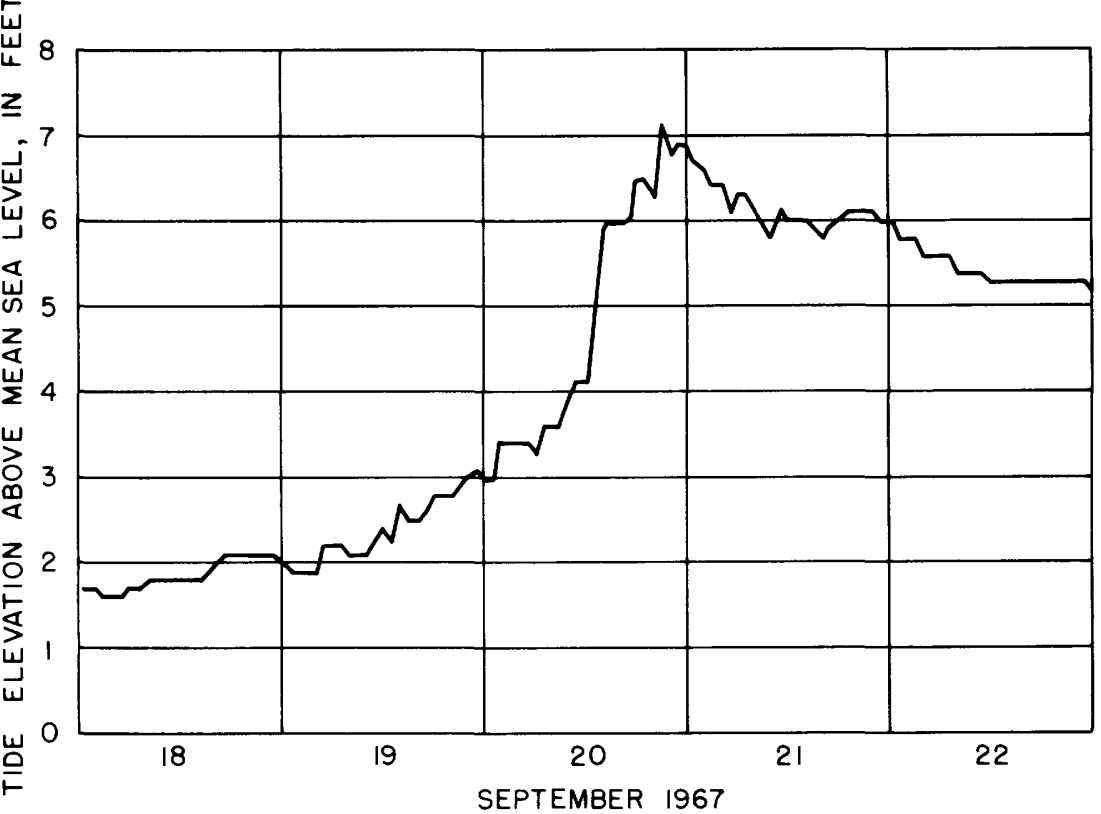

Figure 18. - Tide elevation in Copano Bay at Bayside.

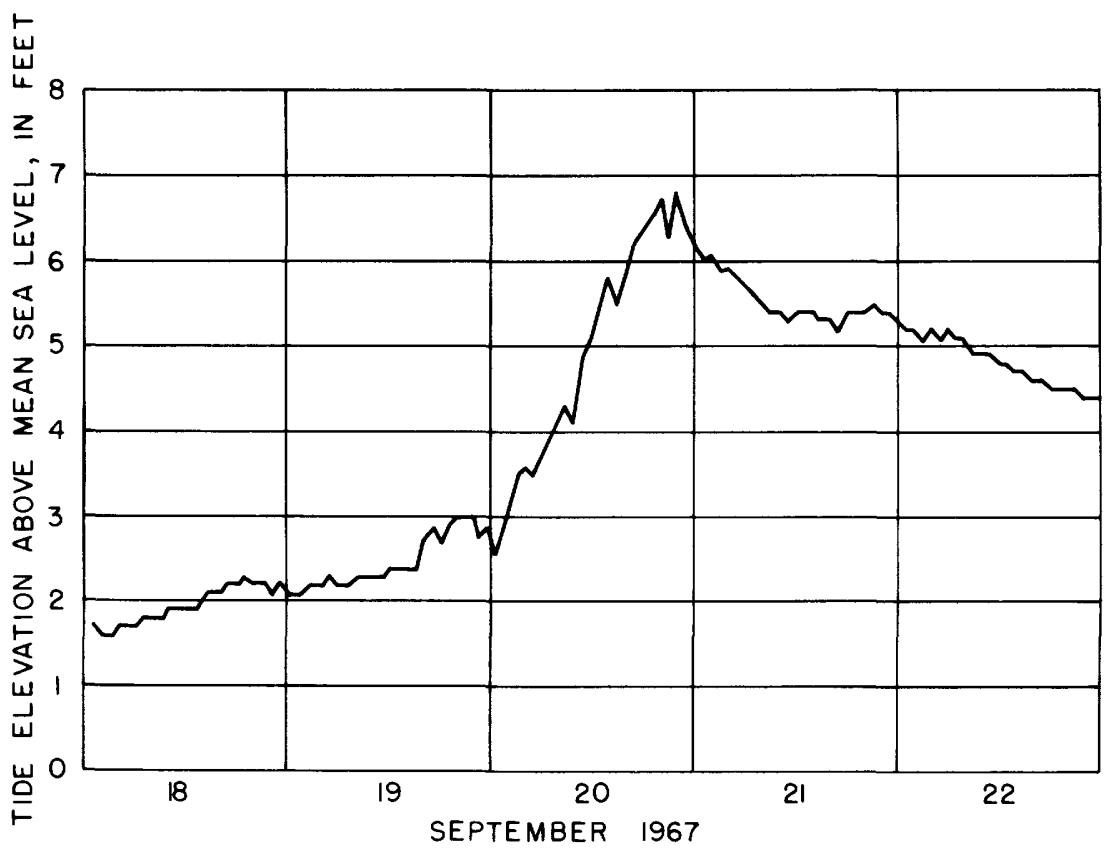

Figure 19.-Tide elevation in Corpus Christi Bay at the tidal basin. 


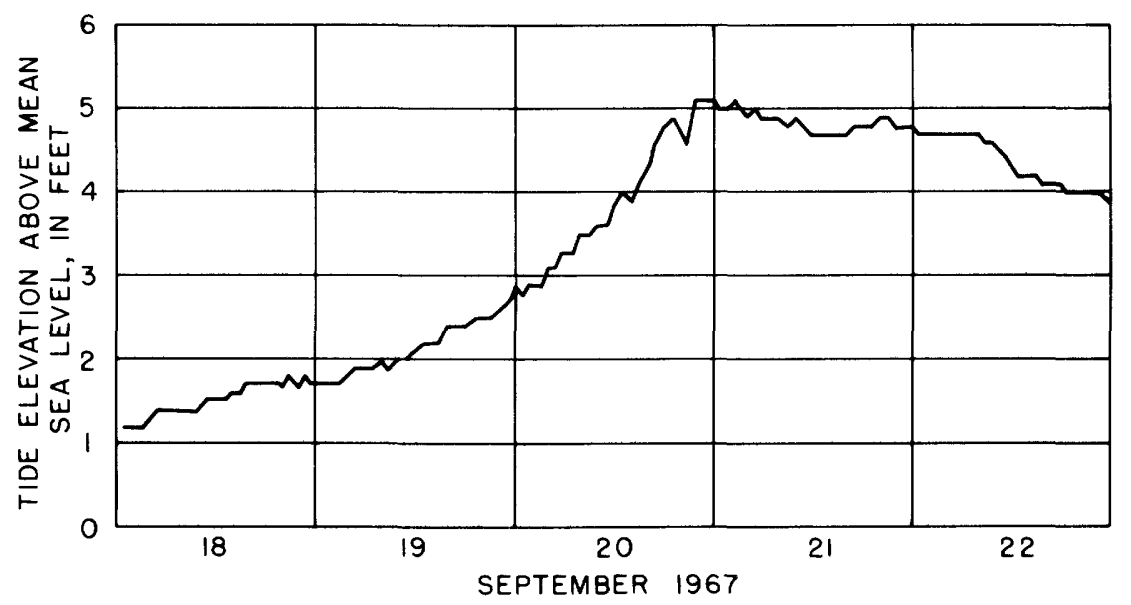

Figure 20.-Tide elevation in Corpus Christi Bay at the Naval Air Force Station.

Escondido Creek, which drains 72.4 square miles above the streamflow station at Kenedy in Karnes County, had very heavy runoff as a result of antecedent conditions in the watershed. Ten floodwaterretarding structures, which partly control the runoff from 36.5 square miles, discharged over the emergency spillways. The weighted-mean rainfall in the watershed above the Kenedy station (site 34) was 17.3 inches, and the peak discharge at this station was $37,000 \mathrm{cfs}$. This discharge is 3.82 times the magnitude of a 50 -year flood.

Because the Escondido Creek watershed is a special study area, detailed information was obtained for two subwatersheds above floodwater-retarding structures. Rainfall and runoff data for these two sites (subwatersheds 1 and 11) are shown in figures 21 and 22. Figure 23 shows rainfall and runoff for Escondido Creek at Kenedy. Some flooding from Escondido Creek did occur. Water overflowed U.S. Highway 281 at Kenedy and inundated some houses in the northeastern part of town.

The San Antonio River was above bankfull stage from Ecleto Creek in Karnes County to the mouth. The volume of runoff contributed by very small tributaries is illustrated by the discharge from Baugh Creek at Goliad (site 36), where a peak discharge of $1,000 \mathrm{cfs}$ was recorded from a drainage area of 3.02 square miles.

The downstream discharge station, San Antonio River at Goliad (site 37 ), had a peak discharge of $138,000 \mathrm{cfs}$ from a drainage area of 3,921 square miles. This discharge is 3.22 times the magnitude of a 50 -year flood, and 4.08 times the previously recorded maximum flood that occurred in 1942 . The peak stage exceeded the previously recorded maximum stage (1942 flood) by 8.8 feet. Available information indicates that the great historical flood of 1869 was several feet higher than the 


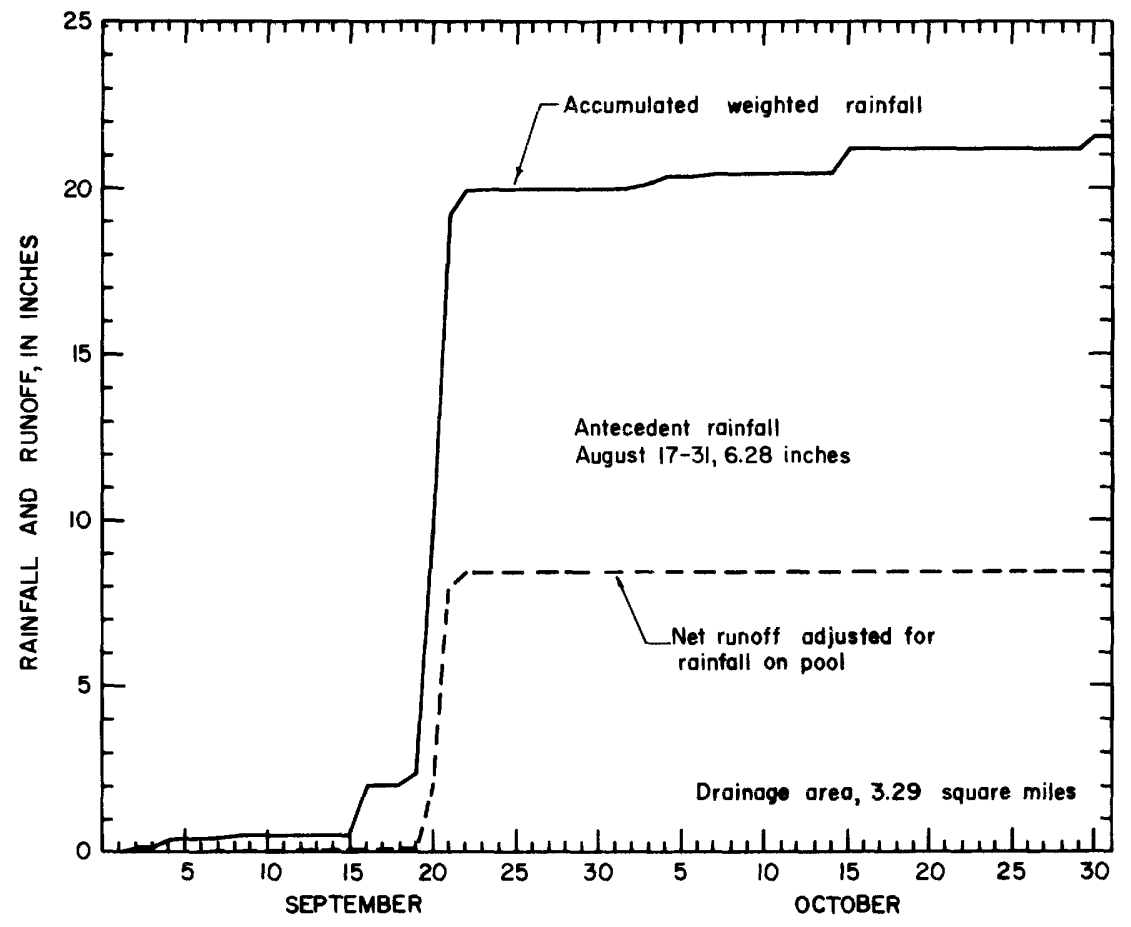

Figure 21.-Accumulated weighted rainfall and net runoff, Escondido Creek subwatershed No. 1.

flood (Beulah) of September 23, 1967. The San Antonio River joins the Guadalupe River just above Tivoli (site 14), where the peak stage was 2.7 feet higher than the previous maximum in 1869 .

Discharge hydrographs for Guadalupe River at Victoria, Coleto Creek near Schroeder, and San Antonio River at Goliad (sites 10, 12, and 37) are shown in figure 24 .

Flooding was severe in the lower part of the basin. All low areas from Goliad to the Gulf of Mexico were inundated, and all highways crossing the river from Goliad to the coast were closed.

\section{MISSION RIVER BASIN}

Extreme flooding occurred throughout the Mission River basin in Bee, Goliad, and Refugio Counties. During the storm period, rainfall of 20 to 25 inches was reported over most of the basin upstream from Refugio. Pettus in Bee County reported an unconfirmed measurement totaling 27.38 inches during the period September $20-24$, which, if correct, is one of the largest amounts reported in Texas. Rainfall in the basin easily exceeded the 2-day, 100-year frequency.

The main stem of the Mission River is formed a few miles upstream from Refugio by the confluence of Blanco and Medio Creeks. On Blanco 


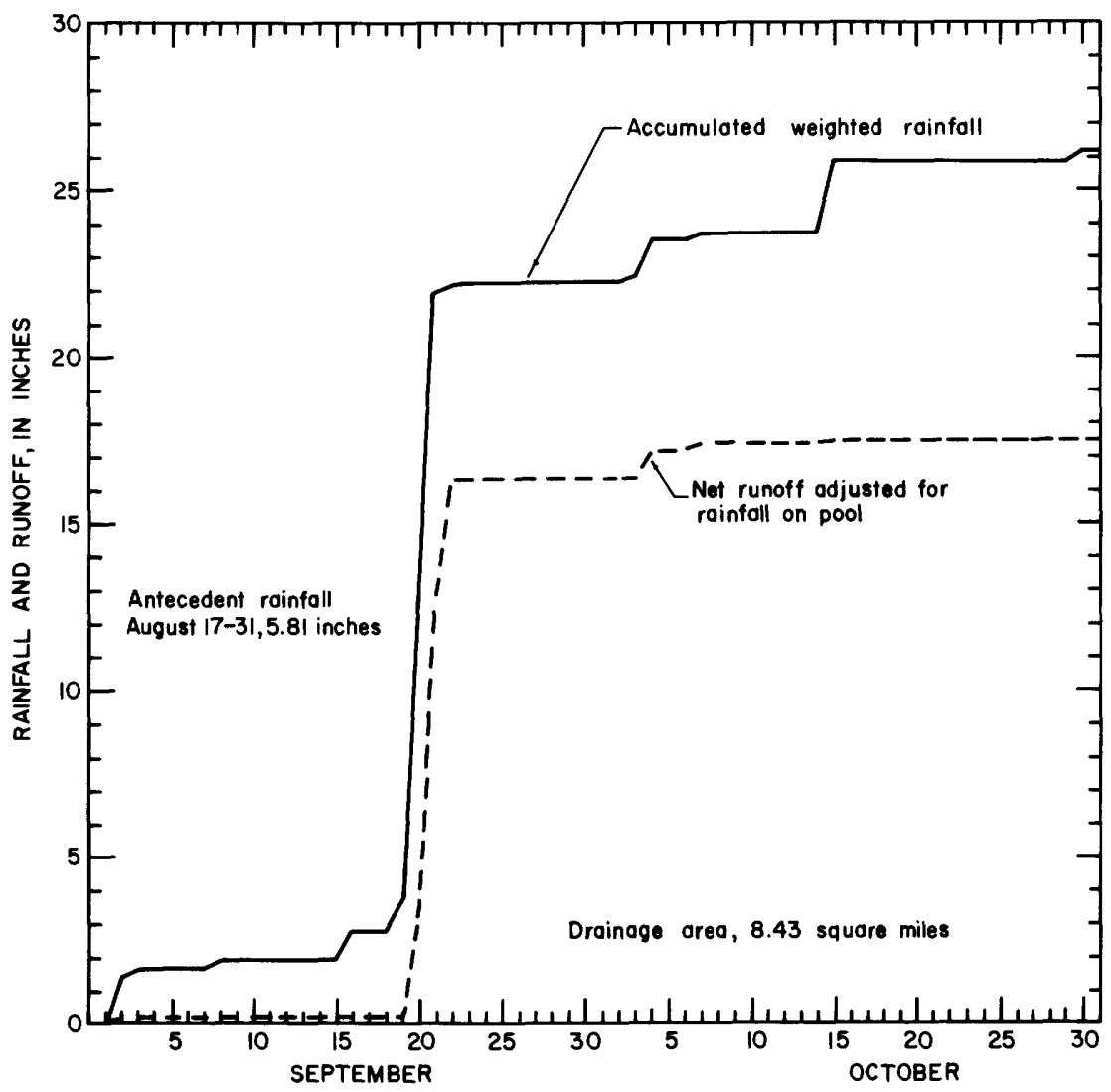

FIGURE 22.-Accumulated weighted rainfall and runoff, Escondido Creek subwatershed No. 11.

Creek, the peak discharge at the miscellaneous site near Berclair (site 38 ) in Bee County was $38,000 \mathrm{cfs}$ from a drainage area of 70.3 square miles. This is 4.28 times the magnitude of a 50-year flood.

Toro Creek, a tributary of Medio Creek, had a peak discharge of 13,$400 \mathrm{cfs}$ from a drainage area of 24.6 square miles at a miscellaneous site near Tuleta (site 39) in Bee County. Medio Creek, which has a drainage area of 204 square miles at the streamflow station near Beeville (site 40), had a peak discharge of $105,000 \mathrm{cfs}$, which is 6.0 times the magnitude of a 50-year flood. The stage for this flood was 7.7 feet higher than the previous maximum in 1919.

At the streamflow station Mission River at Refugio (site 41), a peak discharge of $116,000 \mathrm{cfs}$ from a drainage area of 690 square miles occurred on September 21, the day before Medio Creek crested at the Beeville station. Therefore, the peak at Refugio was principally Blanco Creek water. A secondary peak slightly less in magnitude occurred at 


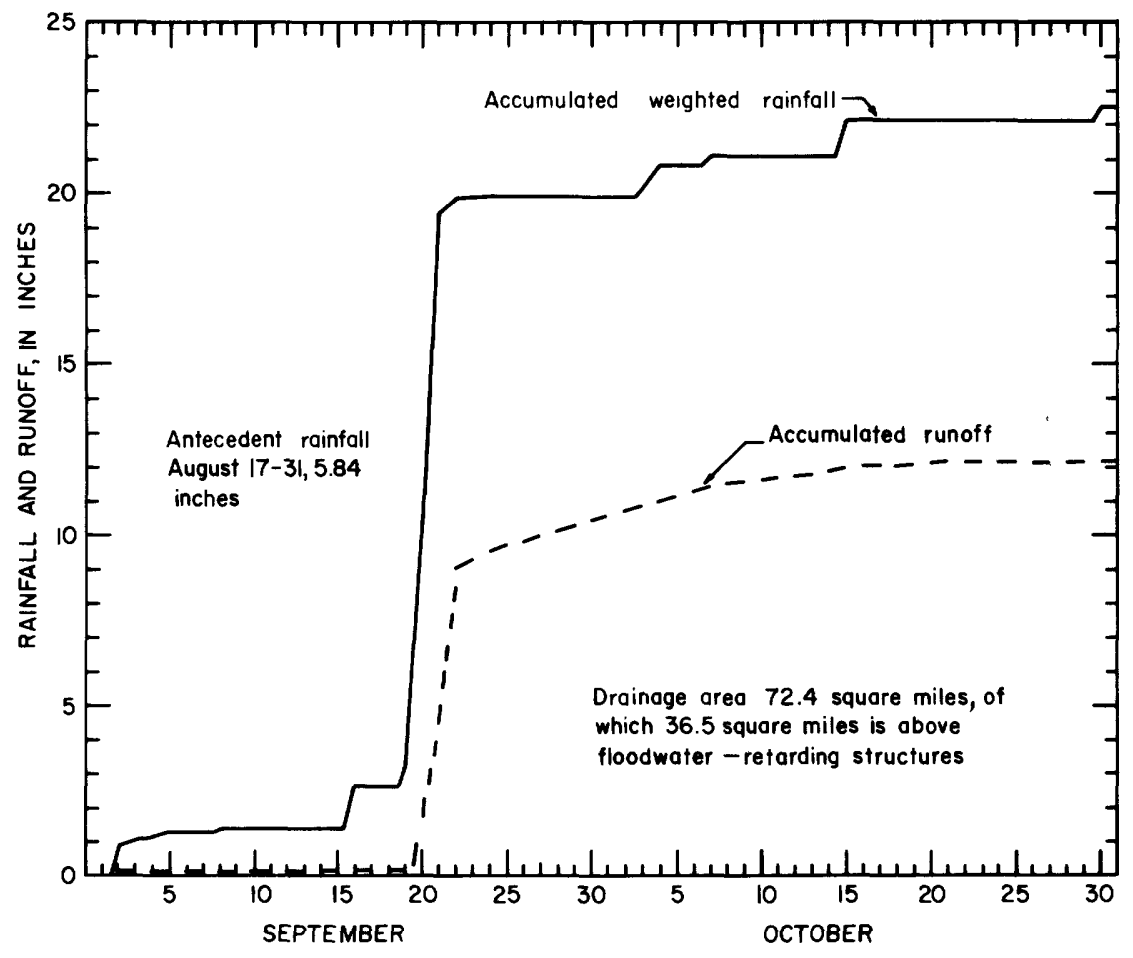

FIGURE 23.-Accumulated weighted rainfall and net runoff, Escondido Creek at Kenedy.

Refugio when the upstream rise from Medio Creek reached the Mission River. The peak discharge for this station is 3.17 times the magnitude of a 50-year flood.

Flood damage was widespread throughout the entire basin. Many rural and urban homes were damaged, and transportation was almost nonexistent because of the many submerged highways. One of the most severely damaged places in the basin was the small unincorporated village of Pettus in Bee County, which was completely inundated by 3 to 5 feet of flowing water from Medio Creek. Five people were reported drowned.

\section{ARANSAS RIVER BASIN}

The Aransas River basin is a small coastal basin having a drainage area of 247 square miles above the streamflow station near Skidmore in Bee County. Rainfall throughout the basin during the storm period was slightly more than 20 inches. Beeville in Bee County reported 18.04 inches in 48 hours ending at 1800 hours on the $22 \mathrm{~d}$, and Sinton in San Patricio County reported 15.21 inches in 48 hours ending at 0800 hours on the 21st. These amounts are equal to or greater than a 2-day, 100year storm. 


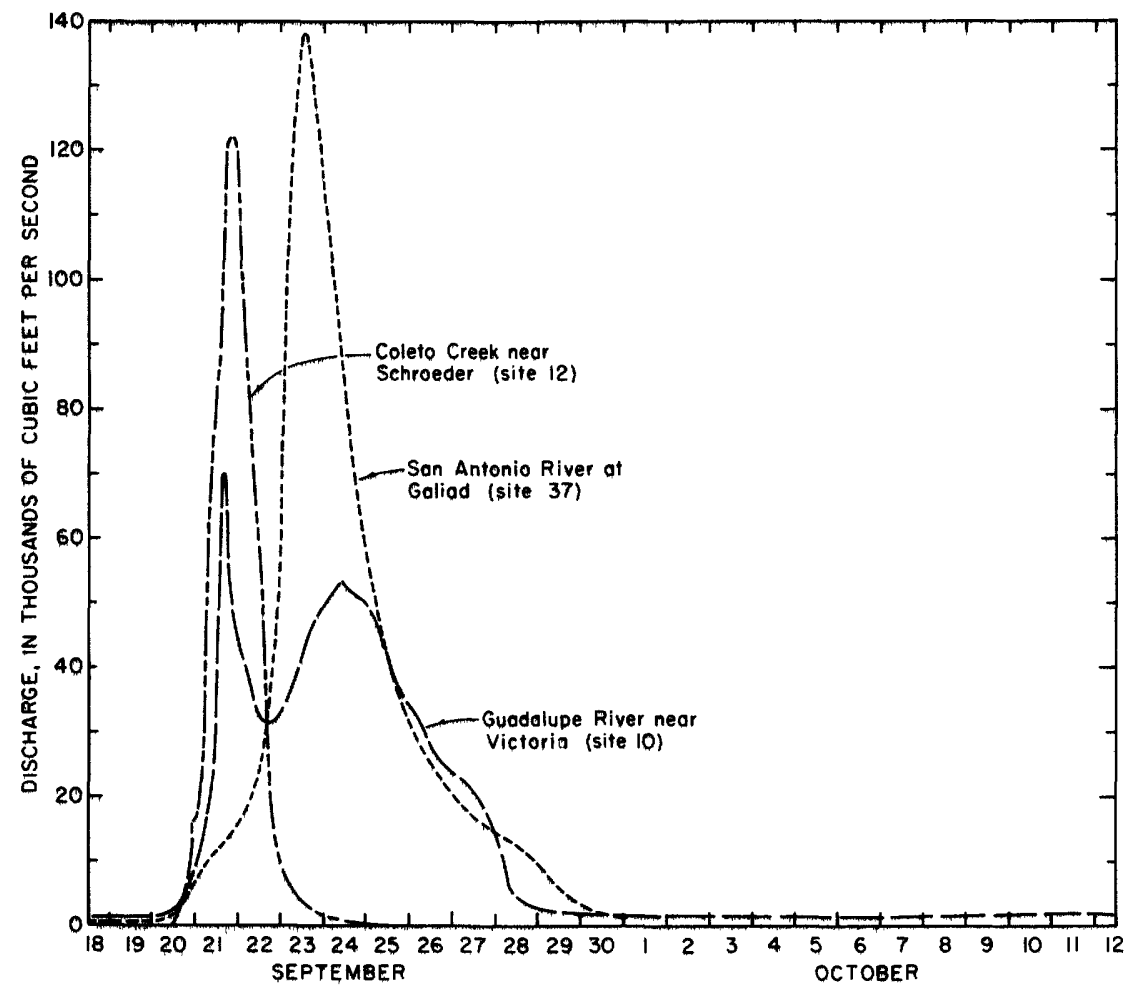

Figure 24.-Discharge hydrographs for Guadalupe River at Victoria, Coleto Creek near Schroeder, and San Antonio River at Goliad.

Flooding in this basin was almost equal to that in the Mission River basin. The streamflow station Aransas River near Skidmore (site 44) had a maximum discharge of $82,800 \mathrm{cfs}$ from a drainage area of 247 square miles. This discharge is 4.23 times the magnitude of a 50-year flood. The stage for this flood was 9.22 feet higher than the previous maximum since 1914.

Poesta Creek (site 42), a tributary in the upper part of the basin, had a peak discharge of $20,800 \mathrm{cfs}$ from a drainage area of 46.5 square miles. This discharge is 3.02 times the magnitude of a 50-year flood.

A very small stream, Olmos Creek tributary near Skidmore (site 43), had a peak discharge of $325 \mathrm{cfs}$ from a drainage area of 0.58 square mile.

A sample of the magnitude of runoff in ungaged areas was obtained at Papalote Creek near Papalote (site 45) in San Patricio County. The peak discharge at this site was $56,400 \mathrm{cfs}$ from a drainage area of 99.2 square miles. This discharge is 5.19 times the magnitude of a 50 -year flood.

Flooding was extensive in the Aransas River basin. Most of the principal highways were closed for a time, and considerable inundation occurred in both rural and urban areas. 


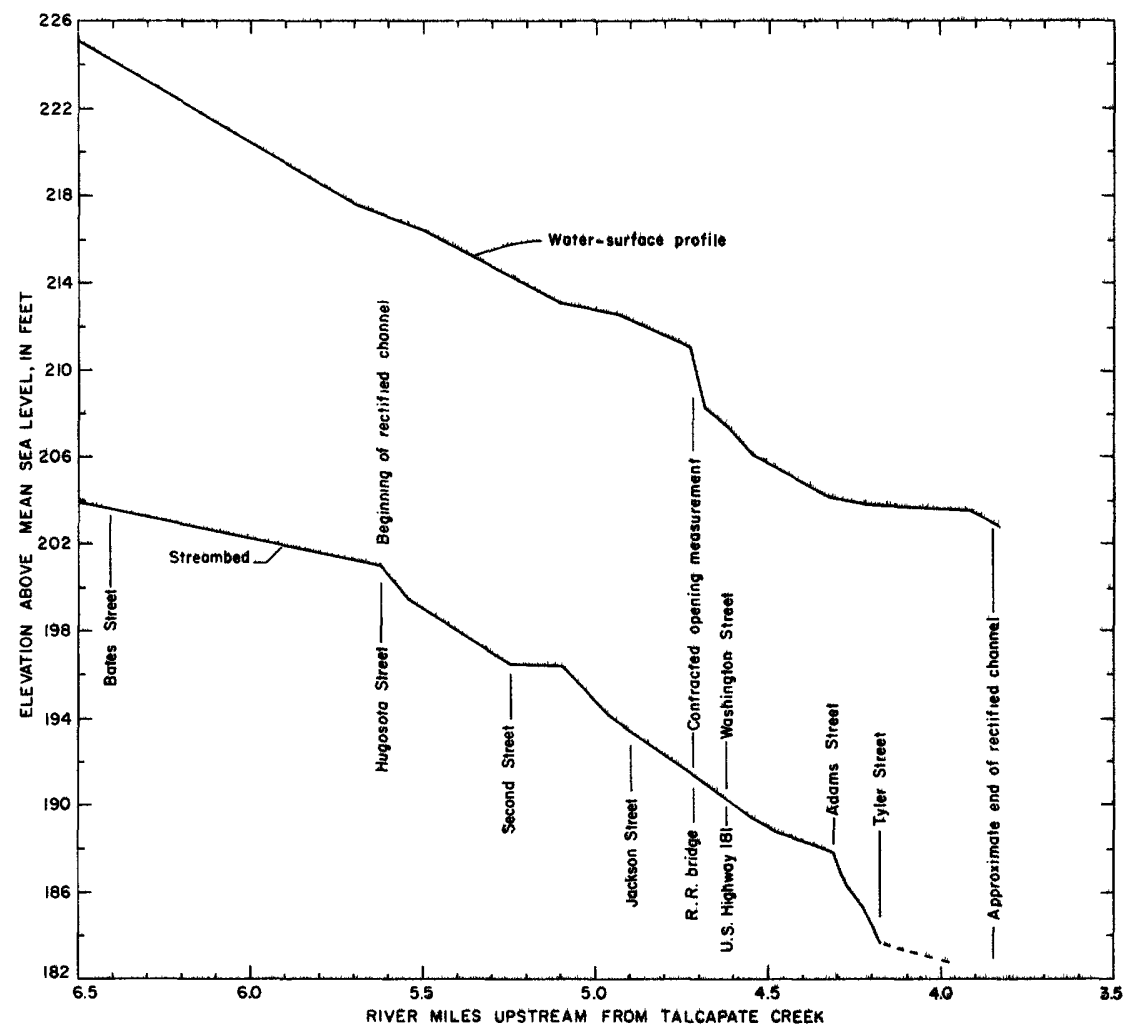

Figure 25.-Flood profile of Poesta Creek at Beeville, September 21, 1967.

Poesta Creek inundated part of Beeville (pl. 2). The depth of inundation at any point can be obtained by using the flood profile (fig. 25) to determine the water-surface elevation and a topographic map to determine the elevation.

Sinton in San Patricio County was completely inundated by water from Chiltipin Creek. Local residents reported that the floodwater in the town was as much as 4 feet deep but had no discernible velocity, so flood damage was less than would have occurred from flowing water.

A form of sheet flow was prevalent, especially in the lower part of the basin. The boundaries of inundation from any particular creek could not be delineated in the classical manner of observing deposits of flood debris because sheet flow extended continuously from one creek to another.

\section{NUECES RIVER BASIN}

The Nueces River basin, which has a drainage area of 16,660 square miles above the downstream discharge station near Mathis (site 61), had the greatest main-stem flood in the lower basin since records began in 1919. Less than one-half of the total drainage area contributed runoff 
to the flood. Runoff from the 5,260 square miles above Cotulla (site 46) on the main stem and from the 3,493 square miles above Derby (site 51) on the Frio River was comparatively low. Furthermore, the Nueces River at Cotulla crested after the large crest had occurred at downstream stations.

Rainfall during the storm period ranged from less than 5 inches at the headwaters to 25 inches in the lower part of the basin. The 2-day, 100year frequency was exceeded at a number of regular weather stations in the lower basin.

The Atascosa River, which drains part of Bexar, Wilson, Karnes, and Atascosa Counties, is tributary to the Frio River at a point just a few miles upstream from the mouth of the Frio River, which is tributary to the Nueces River near a town in Live Oak County appropriately named Three Rivers. In the 1,171-square-mile drainage area upstream from the discharge station at Whitsett (site 56) in Live Oak County, rainfall ranged from less than 10 inches to more than 25 inches, with the heavier amounts occurring in the lower part of the watershed. The greatest 24hour total reported from a regular weather station in Texas was 15.69 inches at Whitsett. This amount greatly exceeds the 100-year frequency.

In the upper part of the watershed, runoff was appreciable but not record breaking. Rutledge Hollow Creek near Poteet (site 54, drainage area $18.3 \mathrm{sq} \mathrm{mi}$ ) and Lucas Creek near Pleasanton (site 55, drainage area $32.8 \mathrm{sq} \mathrm{mi}$ ), both in Atascosa County, had a peak discharge of 1,800 and $2,970 \mathrm{cfs}$, respectively. For Lucas Creek this discharge has a recurrence interval of 8 years.

At the gaging station Atascosa River at Whitsett (site 56), the peak discharge was $121,000 \mathrm{cfs}$. The stage was 0.3 foot higher than the previous maximum in 1881 , and the discharge was 1.70 times the magnitude of a 50-year flood.

Flooding along the Atascosa River was severe from Pleasanton to the mouth. West of Whitsett, State Highway 99 was severely damaged, and near Campbellton, a 3-mile stretch of U.S. Highway 281 was inundated. The railway between Campbellton and Three Rivers was severely damaged. Many of the smaller streams in the watershed caused other roads to be closed at times.

In the Frio River watershed downstream from Derby in Frio County, rainfall ranged from less than 10 inches at Derby to 19 inches at Three Rivers. The 2-day, 100-year frequency was exceeded only in the lower part of the watershed.

At Derby (site 51 ), the peak discharge was only $3,880 \mathrm{cfs}$, which is less than the magnitude of the mean annual flood.

San Miguel Creek near Tilden (site 52), a tributary of the Frio River, in McMullen County had a peak discharge of $13,700 \mathrm{cfs}$ from a drainage area of 793 square miles. Discharge of this magnitude has a recurrence interval of about 4 years. 
At Calliham (site 53) near the McMullen-Live Oak County line, the Frio River had a peak discharge of $57,000 \mathrm{cfs}$. This discharge has a recurrence interval of about 34 years. The stage was about 3 feet less than that during the great flood in 1932.

Flooding was substantial but not record breaking in the Frio River watershed. At Farm Road 99, just downstream from the Frio River gaging station (site 53), the maximum depth over the highway was 7 feet. More than one-half of the total discharge flowed over the left channel bank into Opossum Creek, which for a short distance had more discharge than the Frio River. At this stage, the creek had become an overflow channel of the river.

The drainage area along the main stem of the Nueces River below Cotulla in La Salle County received rainfall ranging from less than 10 inches at Cotulla to about 25 inches near Mathis in San Patricio County. The 2-day, 100-year frequency was exceeded in the lower part of the basin. Mathis reported a 2-day total for September 21-22 of 16.05 inches.

At Cotulla (site 46), a peak discharge of $7,050 \mathrm{cfs}$ was recorded. This discharge, which is less than the mean annual flood, occurred after the crest had passed the downstream stations.

San Casimiro Creek near Freer (site 47), a tributary of the Nueces River, had a peak discharge of $43,200 \mathrm{cfs}$ from a drainage area of 469 square miles. This was a large flood, but the peak stage was 1.4 feet lower than the previous maximum in 1954. At the streamflow station on State Highway 44 west of Freer in Duval County, the width of flow over the highway was 0.9 mile.

On the main-stem Nueces at the gaging station near Tilden (site 48) in McMullen County, the peak discharge was 76,500 cfs. This discharge has a recurrence interval of 45 years. The stage was the greatest known since 1902 and was about 0.1 foot higher than the previous maximum in 1946. In this area, the volume of water contributed by very small streams is indicated by Plant Creek near Tilden (site 49), where a peak discharge of $220 \mathrm{cfs}$ occurred from 0.36 square mile.

The last streamflow station on the main stem above the mouth of the Frio River is at Simmons (site 50) in Live Oak County. The peak discharge at this station was $72,000 \mathrm{cfs}$. The peak stage for this flood was about 0.3 foot lower than the previous maximum in 1919 .

At Three Rivers (site 57) in Live Oak County, the combined flow of the Atascosa and Frio Rivers merged with the Nueces to produce the greatest flood since at least 1875 . A peak discharge of $141,000 \mathrm{cfs}$ occurred on September 23, 1967. This discharge is 1.76 times the magnitude of a 50 -year flood and is 1.66 times greater than the previous maximum, which occurred in 1919 . The 1919 stage was exceeded by 3.2 feet.

Flooding in the town of Three Rivers, which has a population of approximately 2,000 , was nearly catastrophic. The entire business section, 
as well as most of the residential area, was inundated with floodwaters up to 6 feet deep. The depth of inundation was made vividly evident by an ugly deep ring of oil that adhered to the surface of practically every building in town. This oil ring was later found to be about threequarters of a foot below the peak. All traffic was stopped by floodwater. Scouring action sank an internal pier, and two spans dropped from the northbound lane of a bridge on U.S. Highway 281 south of town. Flood damage was heavy in all parts of the lower Nueces basin, but the damage at Three Rivers was the most severe.

The flood at Three Rivers would have been slightly greater had the runoffs from the Atascosa, Frio, and Nueces Rivers reached the site simultaneously. Actually, the Atascosa water probably reached the site several hours before the flood crest of the Frio arrived. The maximum upstream flood crest on the Nueces did not arrive until about 43 hours after the peak on September 23. Flood hydrographs for Nueces River at Simmons, Frio River at Calliham, Atascosa River at Whitsett, and Nueces River near Three Rivers are shown in figure 26.

There is an increase of 1,056 square miles in drainage area between the discharge station at Three Rivers and Wesley E. Seale Dam, which impounds Lake Corpus Christi. This drainage area had a recorded rainfall ranging from more than 15 inches to about 25 inches. A rancher who lives 1 mile south of Dinero in Live Oak County reported that he had measured 34 inches of rainfall during the period September 20-23, 1967.

A sample of the peak rate of flow from this ungaged area was obtained at two sites. Sulphur Creek (site 58), which joins the Nueces River on the east bank near Oakville, had a peak discharge of $43,600 \mathrm{cfs}$ from a drainage area of 71.1 square miles at a site east of Three Rivers. Ramirena Creek (site 59), which drains 84.4 square miles of southern Live Oak County, had a peak discharge of $20,500 \mathrm{cfs}$ at a site south of George West. Evidence of a high runoff rate was noted at other creeks in the area, particularly on Gamble Gully and La Parra Creek. Computations indicate that the peak inflow rate into Lake Corpus Christi was produced by the combined flow of these relatively small creeks and that the peak rate of inflow occurred prior to the arrival of the flood crest of the main-stem Nueces River.

Discharge from Lake Corpus Christi was partly controlled by manipulation of the 60 tainter gates in Wesley E. Seale Dam. These gates were operated throughout the flood to allow the least damage possible, both from backwater flooding upstream and from releasewater flooding downstream. The lake (site 60) had a peak elevation of 94.82 feet (contents 320,000 acre-ft), which is the highest stage since the present dam was completed in 1958. The peak discharge from the lake, at about 1800 hours on September 24, was computed to be $138,000 \mathrm{cfs}$. 


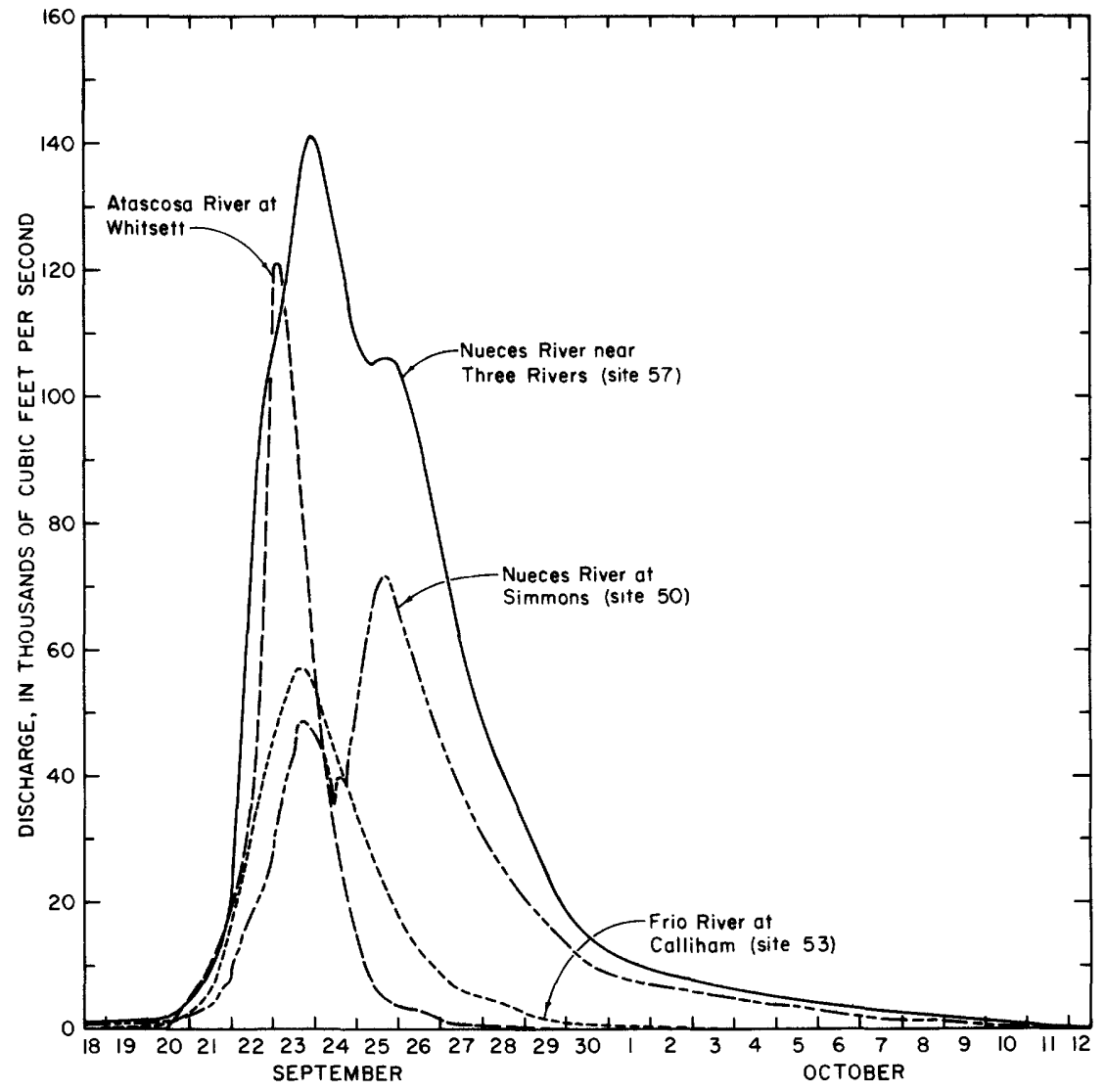

Figure 26.-Discharge hydrograph for Nueces River at Simmons, Frio River at Calliham, Atascosa River at Whitsett, and Nueces River near Three Rivers.

At that time, 54 of the gates were fully open and the other six were partly open.

The discharge station (site 61), 0.6 mile downstream from the dam, had a peak stage of 47.7 feet, which is 7.7 feet higher than the previous maximum in 1919. This discharge of $138,000 \mathrm{cfs}$ is 2.38 times the discharge of the 1919 flood. Discharge hydrographs for Nueces River near Three Rivers and Nueces River near Mathis, are shown in figure 27.

State Highway 359 and the Southern Pacific Railroad were submerged just downstream from the dam for several days. Farther downstream, State Highway 9 and U.S. Highway 77 were also closed. Some homes in Corpus Christi and Calallen suburbs were inundated along Nueces Bay, which had a substantial rise as a result of floodflow from the Nueces River. 


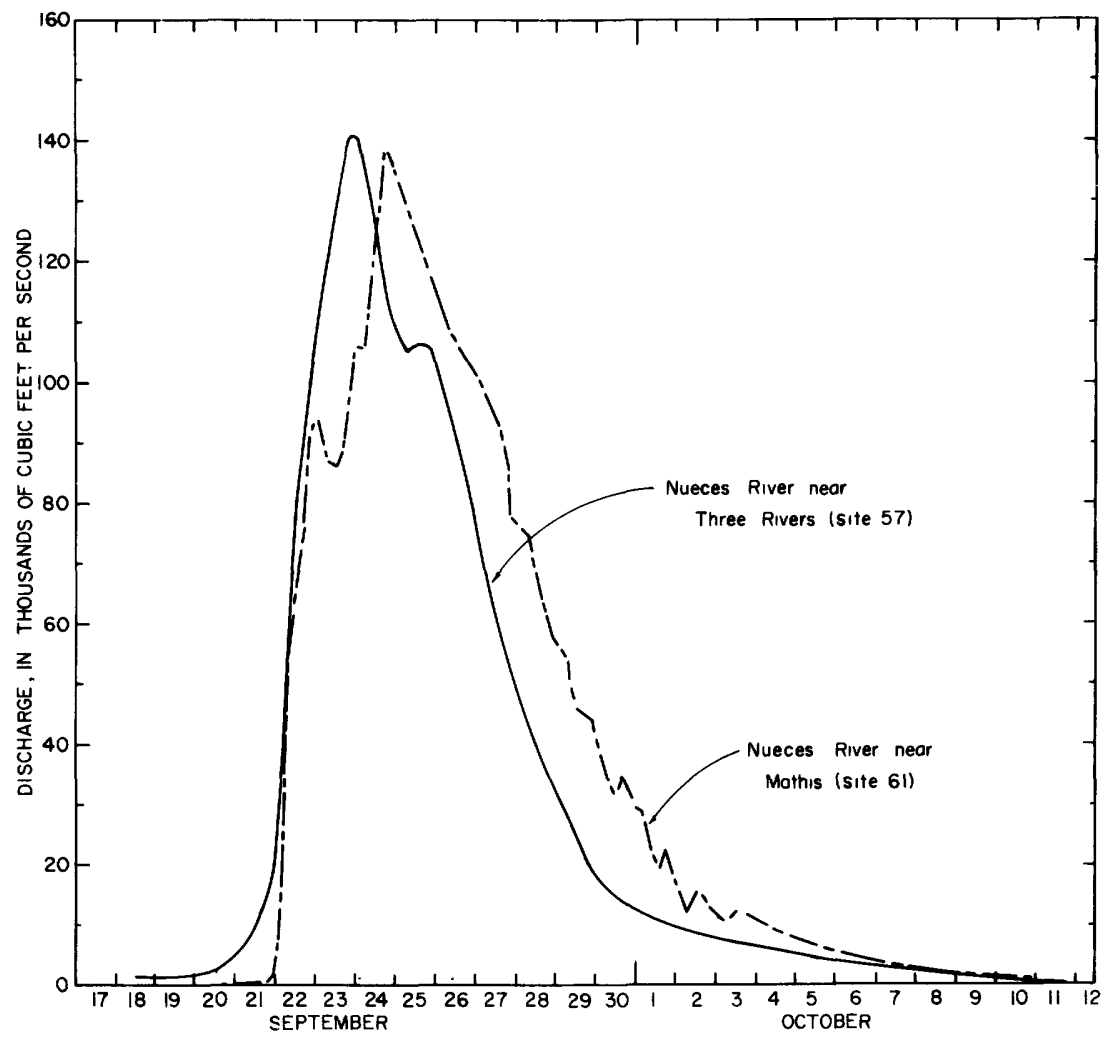

Figure 27.-Discharge hydrograph for Nueces River near Three Rivers and Nueces River near Mathis.

\section{COASTAL AREA BETWEEN THE NUECES RIVER AND THE RIO GRANDE}

The coastal area between the Nueces River and the Rio Grande totals 10,442 square miles, of which 5,179 square miles is probably noncontributing. This area is relatively flat, having a maximum elevation of less than 200 feet above sea level. Total rainfall in the contributing part of the coastal area ranged from 10 to 25 inches during the storm period.

The coastal area between the mouth of the Nueces River at Corpus Christi Bay and the mouth of Los Olmos Creek at Baffin Bay is a 3,576square-mile drainage area, of which 273 square miles is probably noncontributing. This 273 square miles consists mainly of many small depressions, known locally as water holes. The rest of the area is drained by a fairly well developed system of streams that ultimately discharge into Corpus Christi or Baffin Bays. During this flood, some overland flooding occurred as water overflowed from one watershed to another in the lower part of the area. 
Oso Creek (site 62), which discharges into Nueces Bay, had a peak discharge of $3,620 \mathrm{cfs}$ at a site near Violet on State Highway 44 in Nueces County from a drainage area of 45.8 square miles. This vicinity was described as a "sea of water," with water overflowing the highway at many places. At one time, State Highway 44 was the only access road to Corpus Christi. Much of the area east of a line from Mathis to Kingsville to Falfurrias, a distance of about 50 miles, was reported to have been covered by sheetflow as much as 2 feet deep.

San Fernando Creek is one of the principal streams that ultimately discharges into Baffin Bay. A tributary of this creek, San Diego Creek, had a peak discharge of $14,000 \mathrm{cfs}$ from a drainage area of 319 square miles above the discharge station at Alice (site 65) in Jim Wells County. This discharge has a recurrence interval of 44 years. A short distance downstream from the mouth of San Diego Creek, San Fernando Creek (site 67), which has a drainage area of 507 square miles, had a peak discharge of $16,900 \mathrm{cfs}$. This is slightly greater than the 1962 flood, which was the greatest since 1949 .

Runoff from 150 of the 188 square miles of intervening drainage area between the San Diego and San Fernando stations is partly controlled by Lake Alice on Chiltipin Creek (site 66). This reservoir was completed in 1965 and has a capacity of 2,780 acre-feet below the siphon spillway. The maximum contents during this flood was 4,150 acre-feet. Flow from 73.4 square miles above Lake Alice is partly controlled by six floodwater-retarding structures having a combined total capacity of 15,690 acre-feet below the flood spillways.

A short distance downstream from the streamflow station San Fernando Creek at Alice, part of the flood discharge overflowed into Pintas Creek, a tributary of Petronila Creek, which also discharges into the Baffin Bay system.

Near Kingsville, a small dam on Santa Gertrudis Creek broke, and part of the water overflowed into Tranquitas Creek above Tranquitas Reservoir. This reservoir overflowed on both sides, and part of the water flowed into San Fernando Creek, and part of it flowed back into Santa Gertrudis Creek upstream from the King Ranch dairy dam. The dairy dam reservoir then overflowed on the right side and flowed over into Escondido Creek.

At the sites where these three creeks cross U.S. Highway 77, near Kingsville, flood marks indicate that only moderate rises had occurred and that the flow was well confined within banks. A field estimate of $8,700 \mathrm{cfs}$ was computed for San Fernando Creek at the U.S. Highway 77 crossing, just north of Kingsville. At this site, San Fernando Creek has a nominal drainage area of 627 square miles; however, because of the watershed overflow and interchange, the drainage area is indefinite.

The southernmost stream in Texas that contributes discharge to the Gulf of Mexico is Los Olmos Creek. At the streamflow station just north 
of Falfurrias (site 69) in northeastern Brooks County, the peak discharge was $3,380 \mathrm{cfs}$ from 480 square miles. Local residents reported that water overflowed the divide upstream from the gage and flowed south into Cibolo Creek, which flows through the northern part of Falfurrias. The unit peak discharge, in cubic feet per second per square mile, for the Los Olmos Creek station was only about one-seventh of that for the miscellaneous site (site 70) on Palo Blanco Creek 16 miles west of Falfurrias. Downstream from Falfurrias, the low-water channel of Cibolo Creek joins Palo Blanco Creek, and further downstream at a very shallow lake (Laguna Salado) about 25 air miles inland from Baffin Bay, at an elevation of about 80 feet above mean sea level, Baluarte Creek joins the Palo Blanco Creek system. Outflow from the Laguna Salado of the Palo Blanco Creek system, which drains an area greatly in excess of 1,000 square miles, soon disappears among the sand dunes and does not reach the Gulf of Mexico as surface-water flow.

Floods are such a rare event in these sandy areas that Palo Blanco Creek does not have a defined channel through part of Falfurrias. At a site on State Highway 285 about 16 miles west of Falfurrias, Palo Blanco Creek (site 70) had a peak discharge of $16,600 \mathrm{cfs}$ from a drainage area of 343 square miles. On State Highway 285 at a site about $3 \frac{1}{1 / 2}$ miles west of the center of Falfurrias, more than 5,000 cubic yards of fill was required to repair the washout created by Palo Blanco Creek.

In Falfurrias the weather station reported successive daily totals of $2.90,10.00,7.00$, and 5.20 inches of rain during the storm period. The September total exceeded the long-term monthly mean by 28.24 inches.

The city of Falfurrias experienced the worst flood in history. Some residents had to evacuate their homes during the early morning hours of September 22. By that evening, the crest had receded enough for some of them to return home. During the early morning hours of September 23 , the town was almost completely inundated by the combined flow of Cibolo and Palo Blanco Creeks. Some residents report that the south part of town received floodwaters from the overflow of Una de Gato, a very small tributary of Baluarte Creek. Water covered U.S. Highway 281 from north of Los Olmos Creek to south of Baluarte Creek, except for a small hill between Los Olmos and Cibolo Creeks and the hill where the Brooks County courthouse is located. Figure 28 is a profile along U.S. Highway 281, showing the elevation of the water surface. Many business establishments and homes suffered extensive damage, but because of the efforts of the rescue teams, no lives were lost.

Between Baluarte Creek and the Rio Grande, a distance of about 60 miles, there is no defined drainage system. The surface of this noncontributing area of about 4,000 square miles is mostly sandy deposits that can readily absorb normal amounts of rainfall. During this storm, rainfall intensity exceeded the infiltration rate of the sand, and water collected in thousands of shallow depressions. Ponded water inundated 


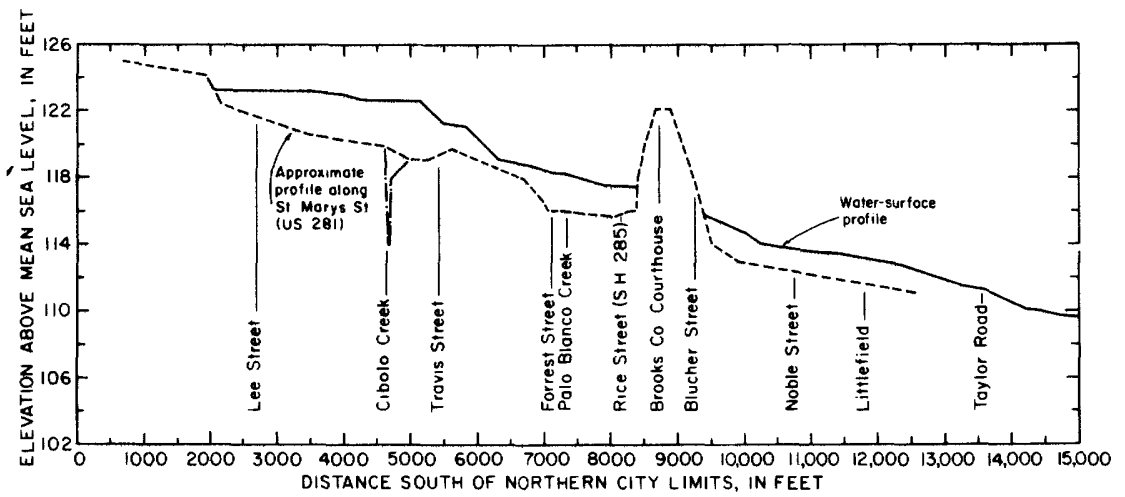

FIGURE 28.-Profile of water-surface elevations along St. Marys Street (U.S. Highway 281) in Falfurrias, September 23, 1967.

U.S. Highway 77 and U.S. Highway 281 at many places and prevented many of the people who had evacuated the area from returning.

Although many of the ponds disappeared rather quickly, many others were still present as late as January 1968, and a few were still present in September 1969 . This remaining ponded water eventually evaporated, and the ponds returned to the normal (dry) condition.

Rainfall decreased west of Encino in Brooks County. Between Encino and La Gloria, in Starr County, there was very little evidence of excessive rainfall. In this area, the sands were apparently able to receive most of the water, although rainfall ranged up to 20 inches.

The area south and east of Encino, which includes parts of Brooks, Kenedy, Willacy, and Starr Counties, was mostly under water. The conditions on the Norias Division of the King Ranch, in Kenedy County, are typical of those in the southeastern part of the basin. Ponded water stood at a maximum elevation of about 20 feet near the Norias headquarters. At the end of October 1967, all ranch roads were closed, and boats were a common means of transportation.

Attempts to drain some of the ponded water into the Gulf of Mexico by ditching and pumping were futile. Some water was removed, but the water level did not decrease appreciably in the ponds because the underlying sands dewatered immediately. More than 2 years elapsed before the depressions became dry and the water table receded to preBeulah levels.

\section{RIO GRANDE BASIN}

Record-breaking floods occurred on the Rio Grande in the reach downstream from Falcon Dam. As a result of the orographic demise of Hurricane Beulah, rainfall in the Rio Alamo watershed was greater than in other areas. Amounts in excess of 35 inches were recorded in this watershed, and amounts ranging up to 20 inches were recorded in the 
adjacent Rio San Juan watershed. These two watersheds constitute more than 85 percent of the approximately 17,700 square miles of contributing drainage area downstream from Falcon Dam. Although there was some runoff in the basin above Falcon Dam, the water was impounded, and no releases were made during the flood.

In the Rio Alamo and Rio San Juan watersheds, antecedent conditions were very favorable for a high percentage of runoff. During the last 2 weeks of August and the first 3 weeks of September, rainfall had been above normal, with totals ranging up to 25 inches recorded in the San Juan watershed during the period August 18-31. Soil-moisture content was high, and all reservoirs in the basin were at or near spillway levels, including the large Marte Gomez Reservoir on the Rio San Juan.

International Falcon Reservoir (site 72) gained 990,885 acre-feet in contents during the period September 19-30, 1967. During this period the peak daily discharge at the station Rio Grande below Falcon Dam (site 73 ) was only $18 \mathrm{cfs}$.

Downstream along the main stem, the first floodwater was contributed by the Rio Alamo from a drainage area of approximately 1,700 square miles. A maximum discharge of $86,500 \mathrm{cfs}$ was recorded at the streamflow station at Ciudad Mier (site 74), Tamaulipas. This discharge alone was sufficient to cause a major flood on the Rio Grande.

The Rio San Juan normally joins the Rio Grande about 23 miles downstream from the mouth of the Rio Alamo. During large floods the Rio San Juan overflows its right bank, and part of the floodwater bypasses both the lower streamflow station and the streamflow station on the Rio Grande near Rio Grande City. During the storm period, rainfall ranging up to 20 inches was recorded in the 13,601-square-mile drainage area of the Rio San Juan. The resultant flood inundated the streamflow station at Carmargo (site 76), Tamaulipas, and floodwater overflowed the right bank on September 22. The peak discharge at the Marte Gomez Dam (site 75), 9.4 miles upstream from the Carmargo station, was $166,000 \mathrm{cfs}$ on September 25.

On the main-stem Rio Grande, a peak discharge of $220,000 \mathrm{cfs}$ was recorded at the streamflow station Rio Grande at Fort Ringgold near Rio Grande City, Tex. (site 77). Under present conditions, this discharge constitutes a great flood with a stage about 10 feet above top of banks. The discharge was only slightly reduced when the crest reached the head of the floodway system near Mission, Tex.

Floodwaters in the Rio Grande are diverted through a series of floodways in both the United States and Mexico. In the United States, part of the excess water is diverted from the river through a system with inlets located approximately 6 miles upstream (Mission Inlet) and 7 miles downstream (Hackney Inlet) from Anzalduas Dam. These channels join at a point 5 miles northeast of Hidalgo to form the Main Floodway which extends eastward about 19 miles to a point about 3 


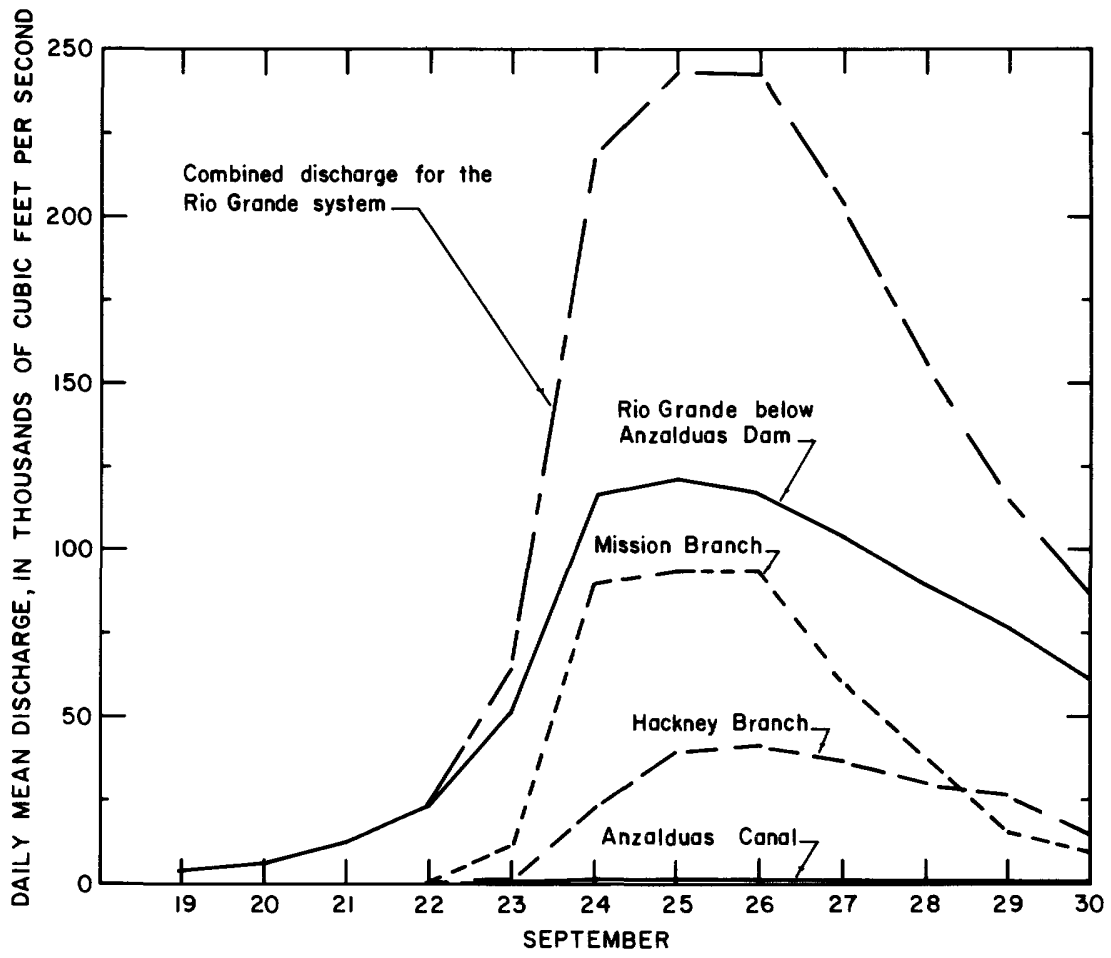

FIGURE 29.-Discharge hydrographs for the United States floodways and the main-stem Rio Grande below Anzalduas Dam.

miles southwest of Mercedes, Tex. At this point, the Main Floodway branches into two channels. The right-hand channel joins the Arroyo Colorado, which discharges into Laguna Madre. The left-hand channel (North Floodway) extends north and east through Cameron and Willacy Counties before it also discharges into Laguna Madre.

This floodway system was completed in 1926. Since that time the system has diverted excess water on nine occasions prior to the Beulah flood; during that storm, the peak discharge through the system was about double the previous maximum in 1932. Figure 29 shows hydrographs of daily mean discharge of the river and the U.S. floodway system below Anzalduas Dam.

The floodway system is designed so that diversions into the Arroyo Colorado can be limited to stay within the capacity of its channel. During this flood, the control structure failed and permitted excessive floodwater to flow into the Arroyo Colorado. As a result, extensive flood damage occurred along the channel.

On the Mexican side of the Rio Grande, there are three principal floodways, which are operated by the Ministry of Hydraulic Resources. 


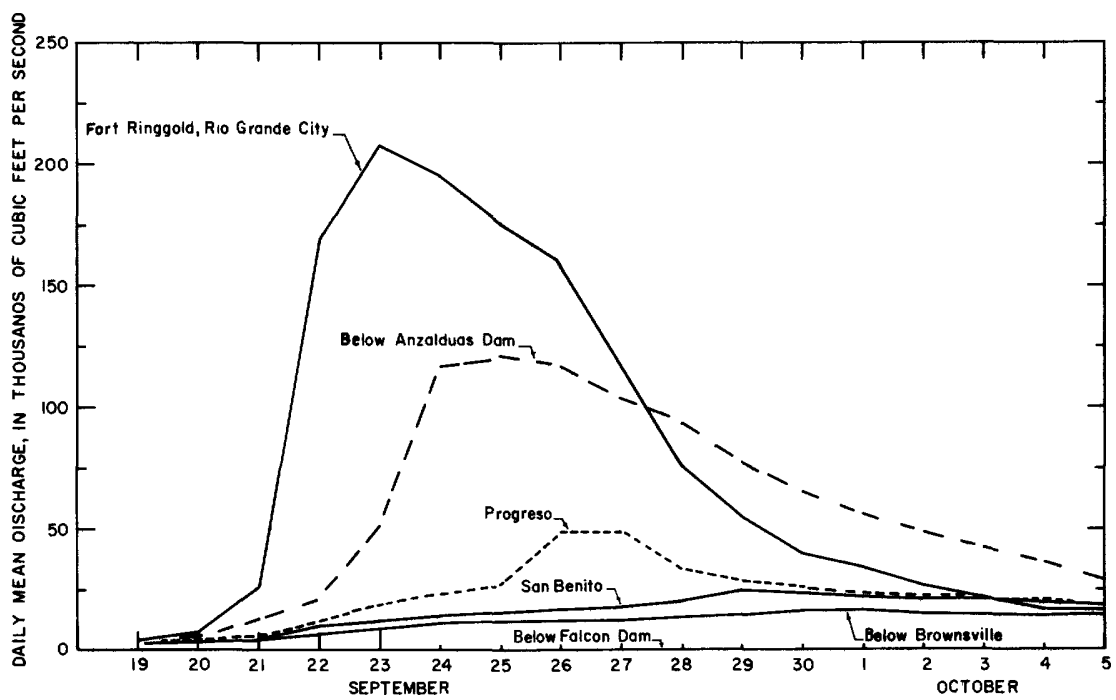

FIGURE 30.-Discharge hydrographs for each main-stem Rio Grande gaging station downstream from Falcon Dam.

These are located about 38 miles (Retamal Heading), 51 miles (San Rafael), and 107 miles (Floodway, No. 2) downstream from Anzalduas Dam. To date, no discharge data have been published by the Ministry of Hydraulic Resources. However, the effectiveness of the entire floodway system is graphically illustrated by figure 30 , which shows the hydrographs of daily mean discharge at each of the main-stem gaging stations below Falcon Dam. A considerable reduction in rate of flow, as well as a reduction in volume of water in the main channel, was attained by the system. The maximum daily mean discharge at the lower Brownsville station (site 93) was 15,500 cfs on October 1, 1967.

A factual comparison of the Beulah flood with previous great floods is nearly impossible because of the many manmade changes in the Rio Grande basin, such as the floodway systems, the Marte Gomez Dam on the Rio San Juan, and the Falcon Dam on the Rio Grande. Discharge records for the United States floodway show that the system carried more floodwater during the Beulah flood than at any time since completion in 1926.

The lower Rio Grande Valley area was damaged severely by the Beulah storm. This damage was inflicted by a combination of hurricane-force winds, excessive rainfall that destroyed fall vegetable crops, and flooding that inundated residential and business areas.

\section{MAGNITUDE AND FREQUENCY OF THE FLOODS}

The magnitude of floods of this report has been described in terms of recurrence interval, ratio to a regional 50-year flood, ratio to the 
previously known maximum discharge, or the number of feet that the maximum previously known stage was exceeded. Each of these standards of comparison provides a means of stating the relative severity of a flood. These methods refer to the maximum instantaneous rate of discharge, or the maximum stage.

The magnitude of a flood may also be stated in terms of volume of runoff, which can be expressed in acre-feet or in inches of runoff from the contributing drainage area.

Expressing the magnitude of a flood in terms of recurrence interval or, more correctly, "probability of exceedance" is the favorite tool of those concerned with the evaluation of the flood potential of a stream when structures located in the flood plain are designed. Using this method, the probable return frequency can be determined by analysis of flood records for gaging stations. Regional flood characteristics are developed from a statistical study of flood experiences on a number of streams. The reliability of calculated return freqencies may be expected to vary with the aerial coverage and the number of years of flood records on which they are based. A fair degree of confidence is indicated for recurrence intervals as great as 50 years; extension of curves beyond that period is not recommended. A flood having a recurrence interval of 50 years has a 2-percent chance of occurring in any year. The regional frequency relations for streams in Texas, presently in use, were developed from records of streamflow collected prior to 1961 and utilize the index-flood concept.

A relatively easy method of determining an implied frequency for a streamflow station is by means of the flood-distribution graph. Figures 31 through 33 are flood-distribution graphs for three selected stations in the Beulah flood area. This method provides a means of rapidly determining the approximate magnitude of a flood by visual inspection on the basis of stage.

The regional flood-frequency curves shown in figures 34 and 35 are based on a Gumbel (1954) distribution of annual maximum discharge experienced prior to 1961 . This method expresses the magnitude of floods in each region in terms of the ratio of the discharge to that of the index flood, which is the mean annual flood or the flood having a recurrence interval of 2.33 years in the Gumbel distribution. Based on this method, the greatest flood experienced during the Beulah storm period, in Texas, occurred at Medio Creek near Beeville (site 40; fig. 34 ), where the peak discharge was 6.00 times the magnitude of the regional 50-year flood.

Special methods must be employed to determine the flood frequency for stations on the main stem of some streams. The main-stem Nueces River and its principal tributary, the Frio River, falls into this special category. Figures 36 and 37 are the frequency relations presently used for these streams. 


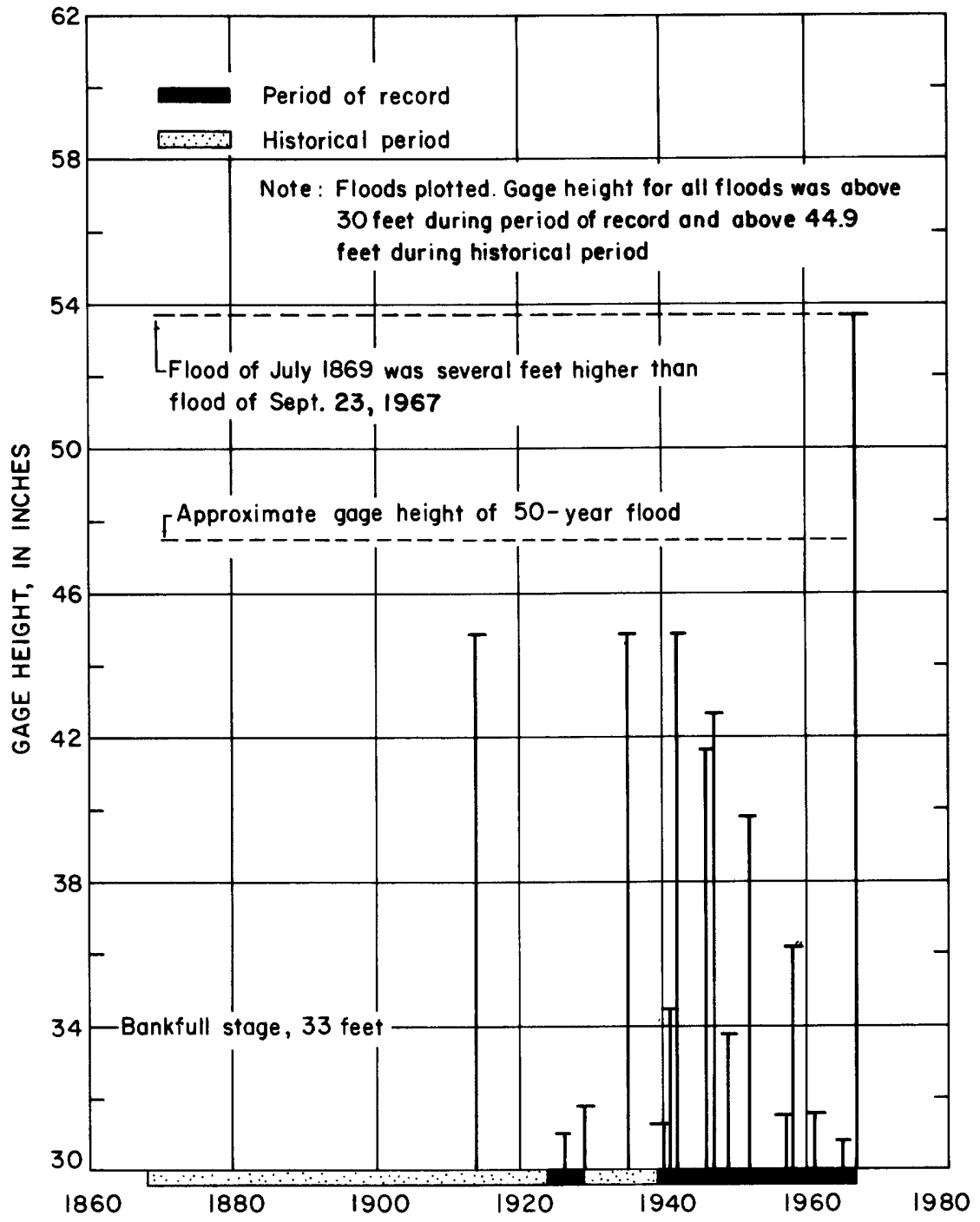

FIgURE 31.-Flood-distribution graph for annual floods, San Antonio River at Goliad.

Some hydrologists prefer to compare the relative magnitude of floods by means of curves that show the relation between peak discharge per square mile and the contributing drainage area. Two of these curves are the Jarvis-Myers curve and the Commons curve. These curves are expressed as: $q=K A^{x}$; where $q$ is the peak discharge per square mile, $K$ is a constant, and $A x$ is the contributing drainage area raised to some power. 


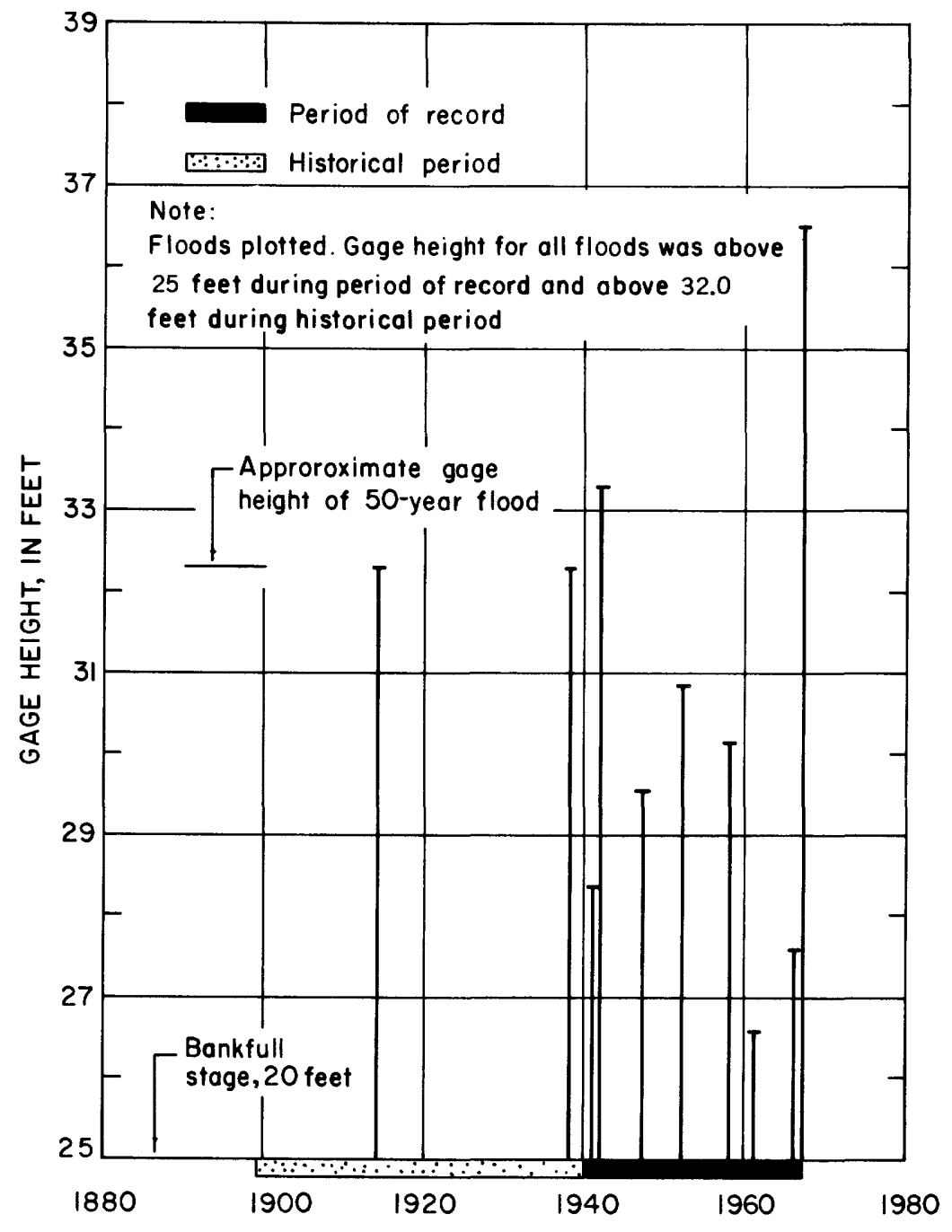

FlGuRE 32.-Flood-distribution graph for annual floods, Mission River at Refugio.

The Jarvis-Myers curve is based on the theoretical concept that the maximum possible peak discharge from 1 square mile is $10,000 \mathrm{cfs}$, and that the peak discharge per square mile will diminish in inverse ratio to the square root of the drainage area.

The Commons curve was derived empirically. It is a curve that encompasses the maximum discharges experienced and the discharges computed from the maximum stages experienced. This curve has been a practical tool for those concerned with the design of structures that must withstand and control major floods. 


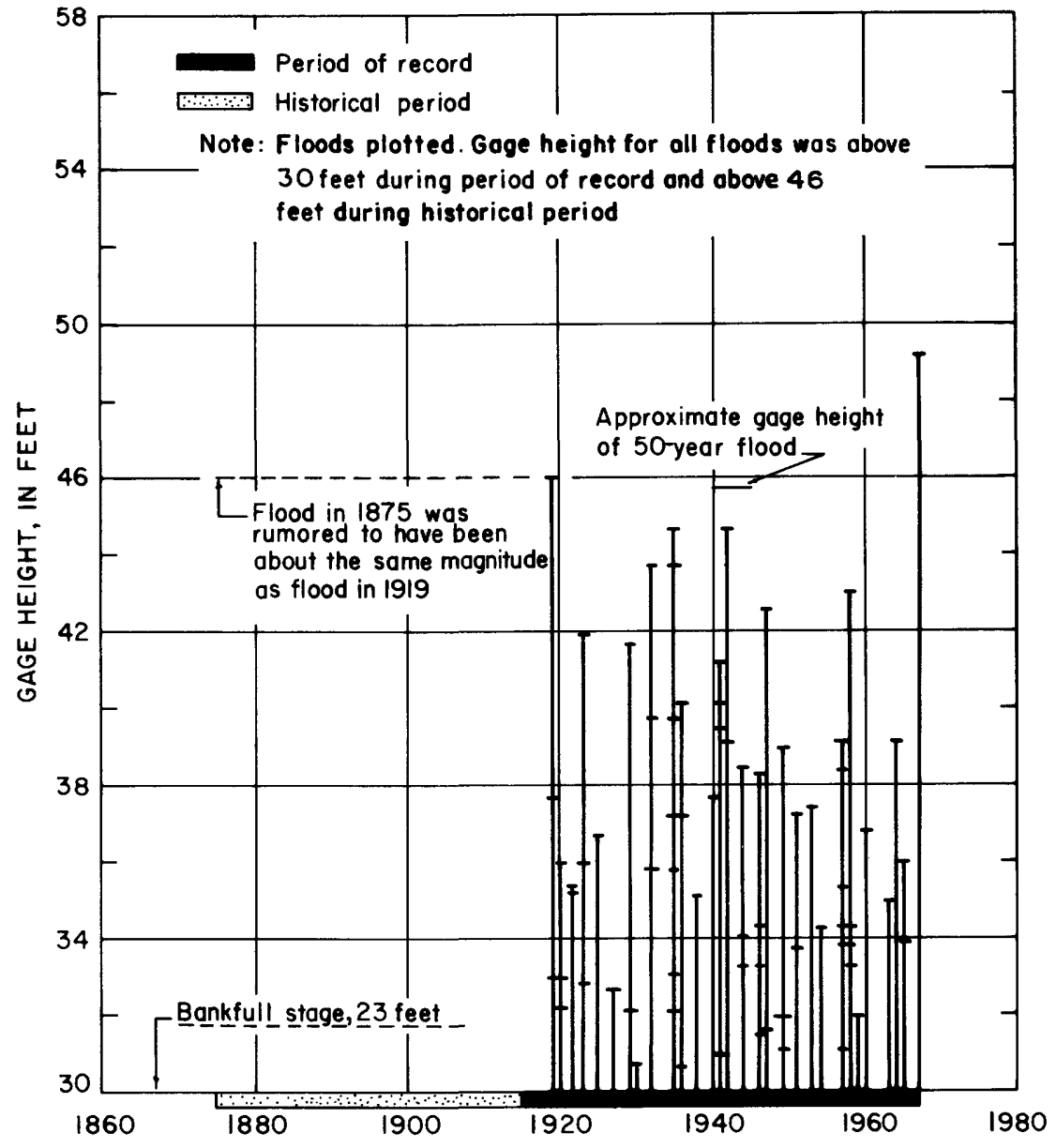

FiguiRe 33.-Flood-distribution graph for Nueces River near Three Rivers. 


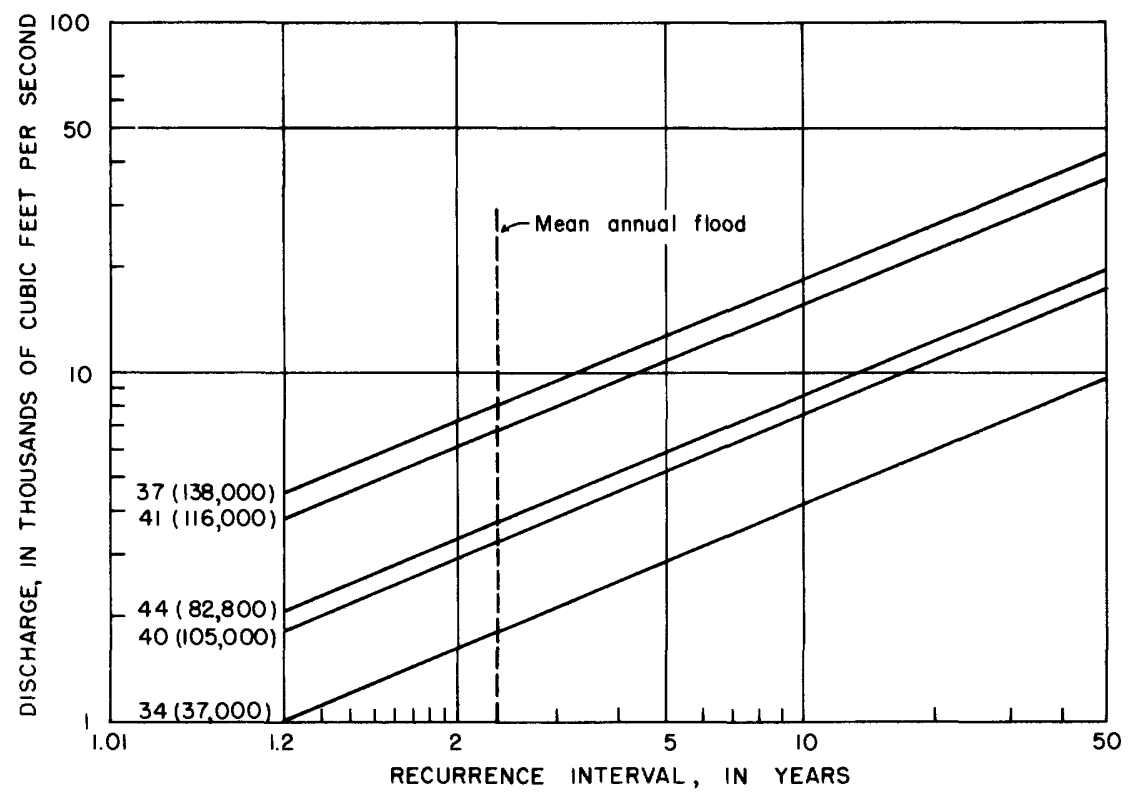

Figure 34.-Regional flood-frequency curves for selected stations. Frequency curves after Patterson (1965). Numbers conform to those in table 17 and on plate 1 . Numbers in parentheses are September 1967 peak discharges.

The unit peak discharges experienced during the Beulah storm period have been plotted on figure 38 to illustrate how these peaks rank in magnitude by these standards. The Jarvis-Myers curve is not really applicable to streams in this area, and the Commons curve includes streams in North Texas. A curve encompassing the maximums experienced during the Beulah storm period is also shown. 


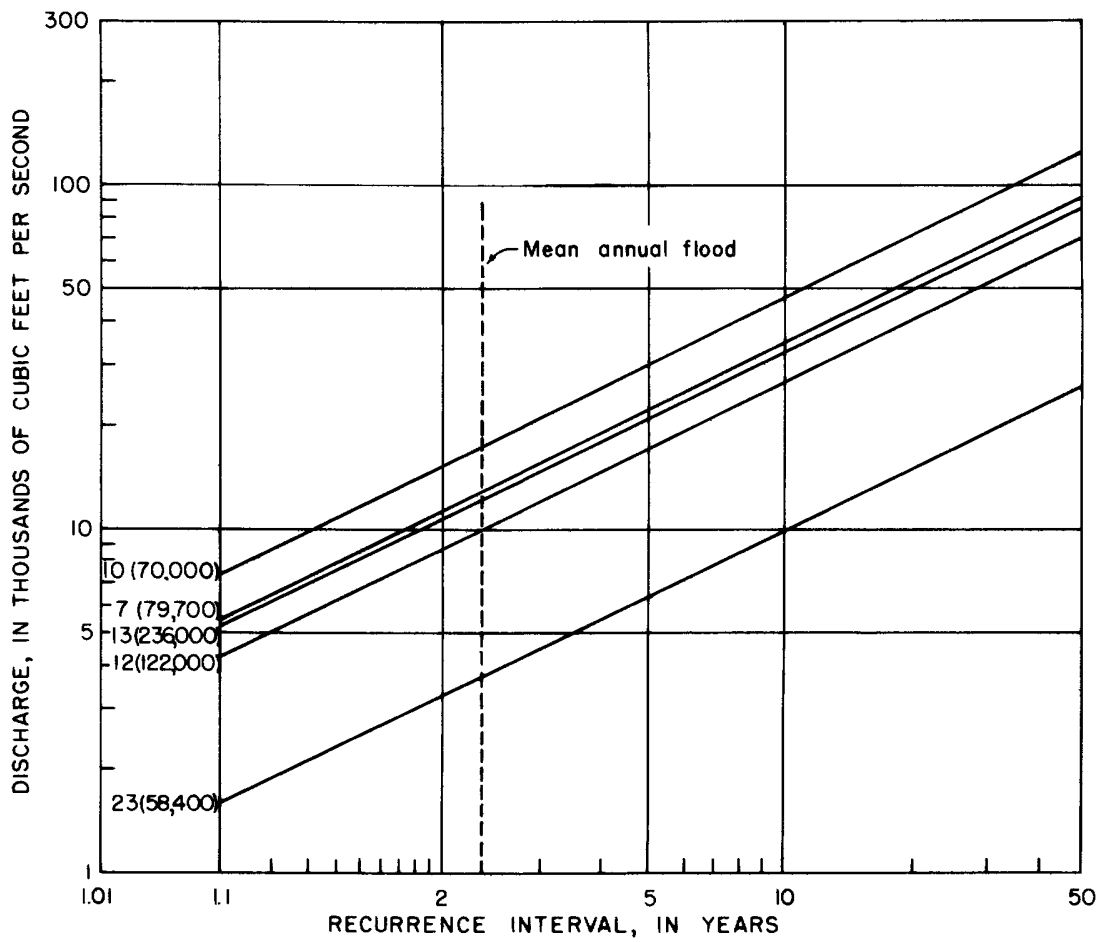

FigURE 35.-Regional flood-frequency curves for selected stations. Frequency curves after Patterson (1965). Numbers conform to those in table 17 and on plate 1 . Numbers in parentheses are September 1967 peak discharges. 


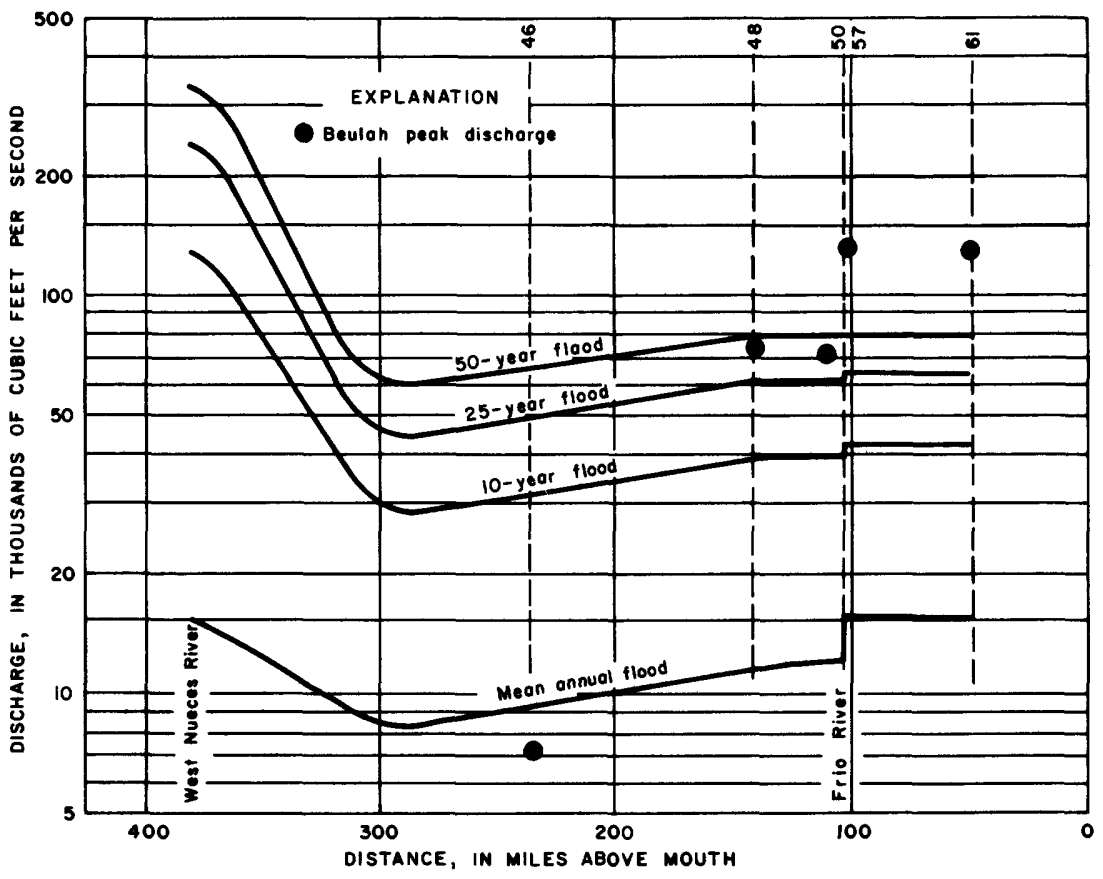

FigurE 36.--Peak discharge on the main-stem Nueces River below West Nueces River and the relation of discharge to distance above the mouth for selected recurrence intervals. Numbers refer to gaging stations in table 17 and on plate 1. Frequency curves after Patterson (1965). 


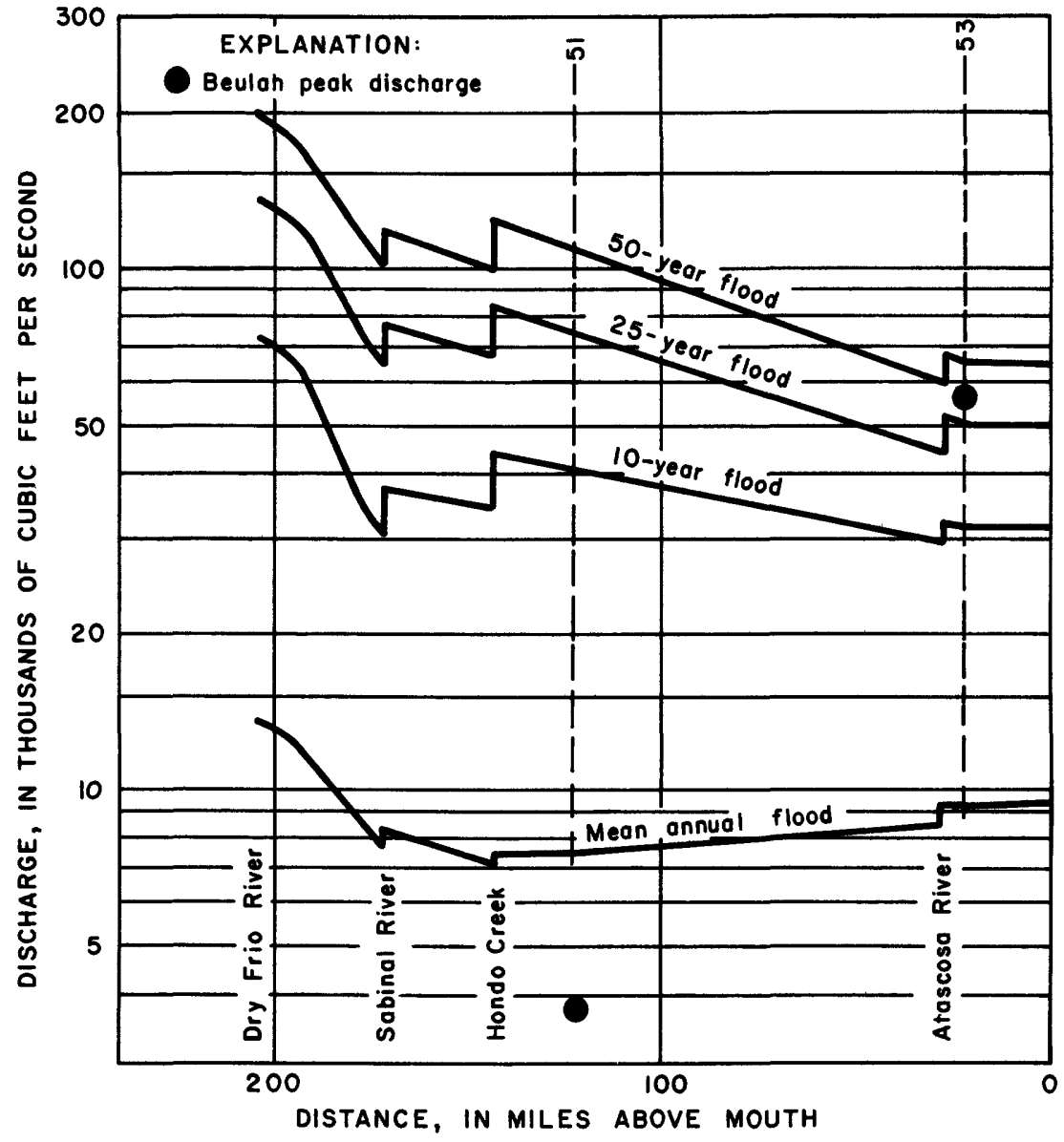

Figure 37.-Peak discharge on the Frio River below Dry Frio River and the relation of discharge to distance above the mouth for selected recurrence intervals. Numbers refer to gaging stations in table 17 and on plate 1. Frequency curves after Patterson (1965). 


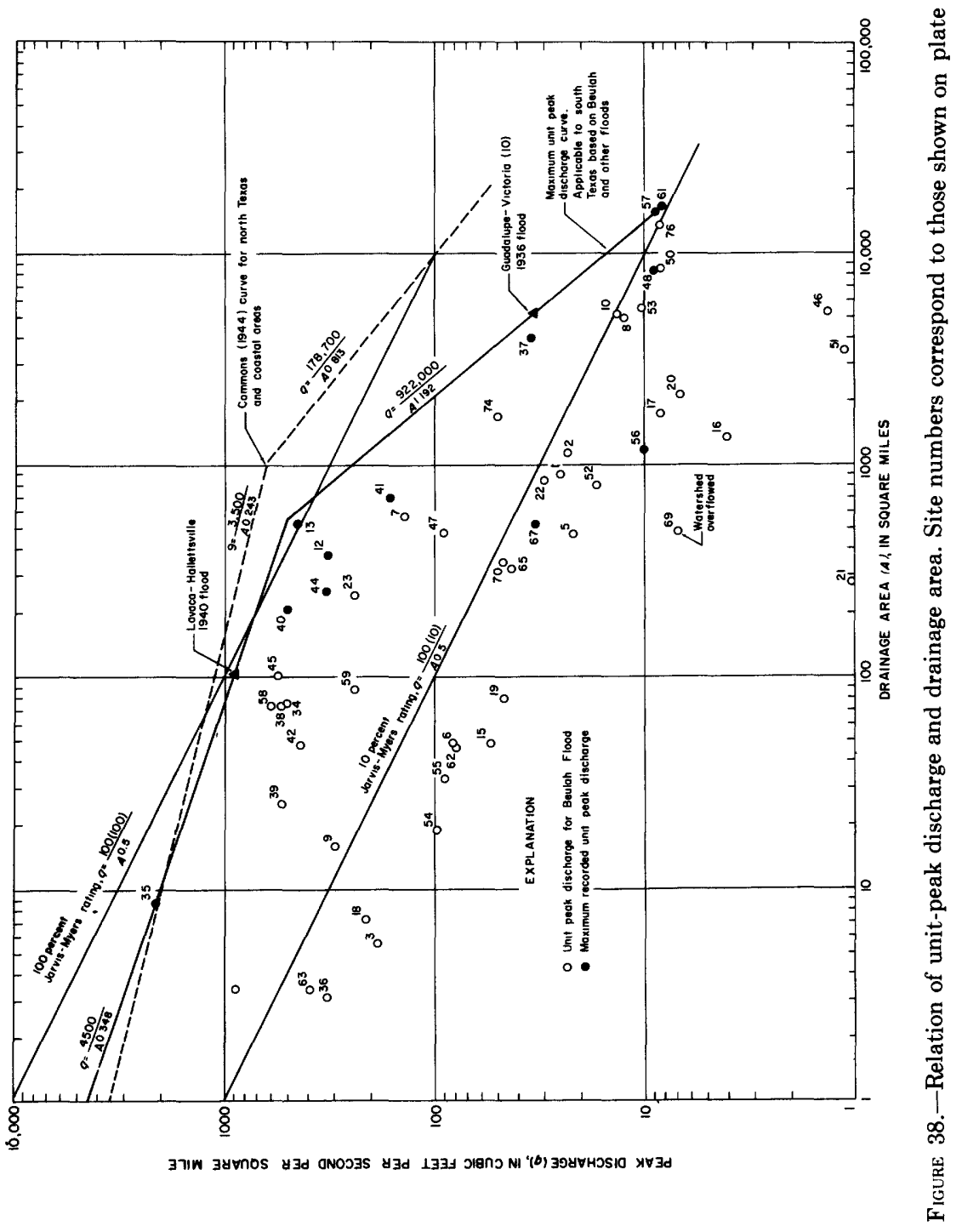




\section{FLOOD VOLUME}

An estimate of the expected runoff volume from a stream is a requisite for those who design water-supply and flood-control reservoirs. The storm at Thrall, Tex., on September 9-10, 1921, (38.2 inches in 24 hours) has been used by many designers since that time as the standard for maximum probable rainfall. Based on the Thrall storm, estimates of runoff ranging up to 17 inches or more have been used.

The maximum runoff recorded during the Beulah storm period was 16.4 inches from 8.43 square miles at Escondido Creek subwatershed No. 11 (site 35) near Kenedy, Tex., with larger watersheds recording less than this amount. Runoff volume in square mile-inches during the Beulah storm period for 24 selected drainage areas is shown as pictographs in figures 39 through 41 . The drainage areas are presented in downstream order to illustrate some of the variability of hurricane storms. These graphs also provide a means of visually comparing total runoff volumes.

The relationship of runoff, in inches, and drainage areas is shown in figure 42 for those watersheds producing more than 1 inch of runoff. The maximum encompassing curve may serve as the basis for a design curve for south Texas. Although no volume-frequency studies for Texas have been published, this curve probably represents a recurrence interval of about 50 to 100 years for a single storm on the basis of rainfall frequency.

For those interested in the total runoff volume expressed in acre-feet, figure 43 shows the relationship of total storm runoff, in acre-feet, and drainage area. The equations for the maximum encompassing curve are similar to those for the maximum unit peak discharge in form and also exhibit the same shape characteristics with a sharp break occurring at the 550-square-mile point.

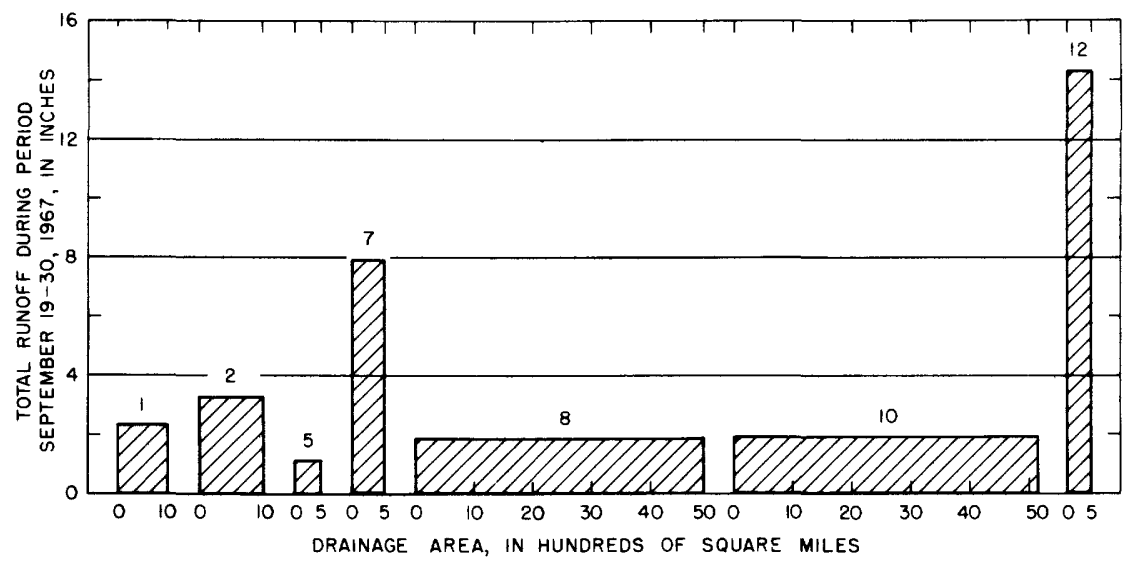

Figure 39.-Runoff volume for selected drainage areas. Site numbers above bars correspond to those shown on plate 1 . 


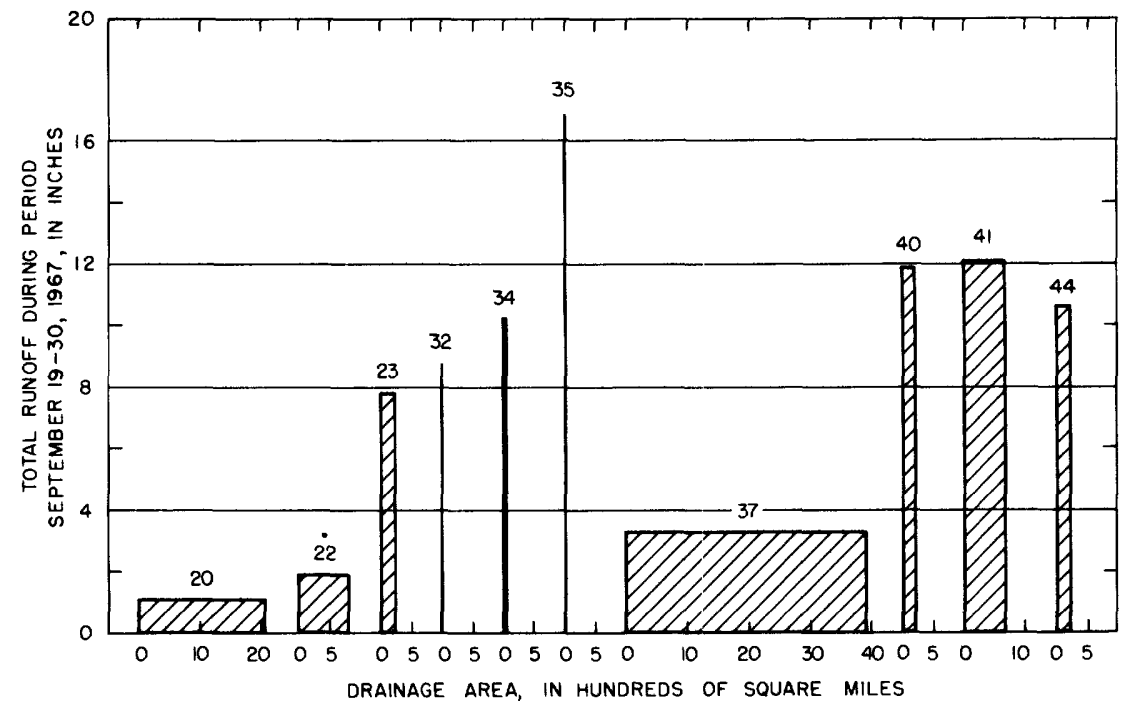

FIGURE 40.-Runoff volume for selected drainage areas. Site numbers above bars correspond to those shown on plate 1.

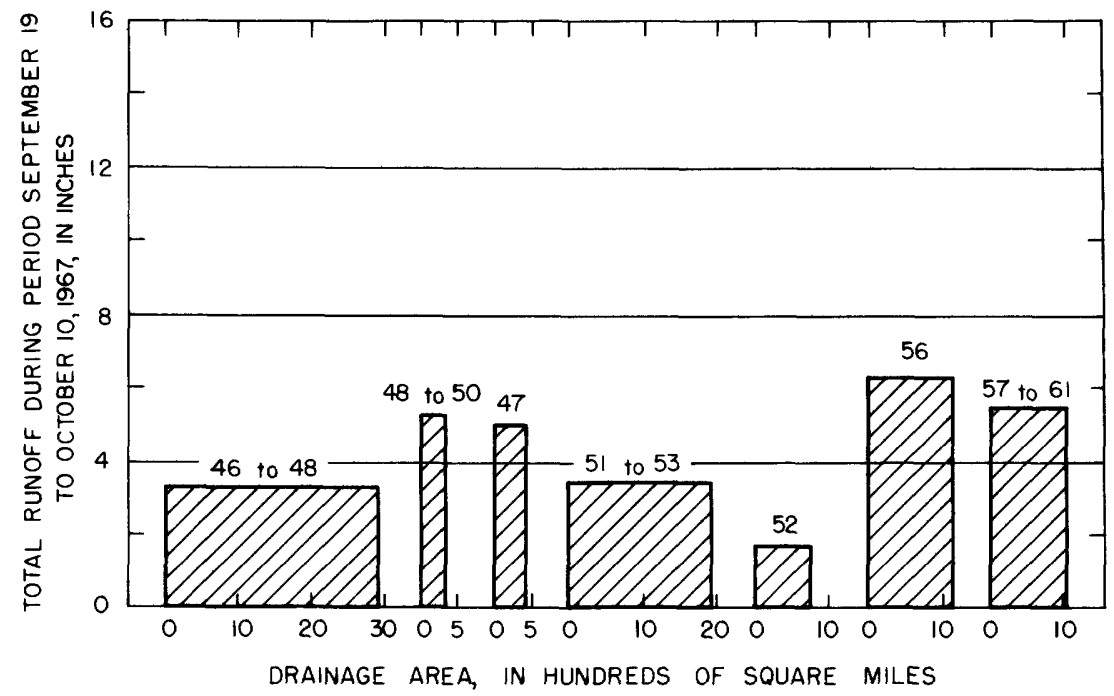

FIGURE 41.-Runoff volume for selected drainage areas. Site numbers above bars correspond to those shown on plate 1. 


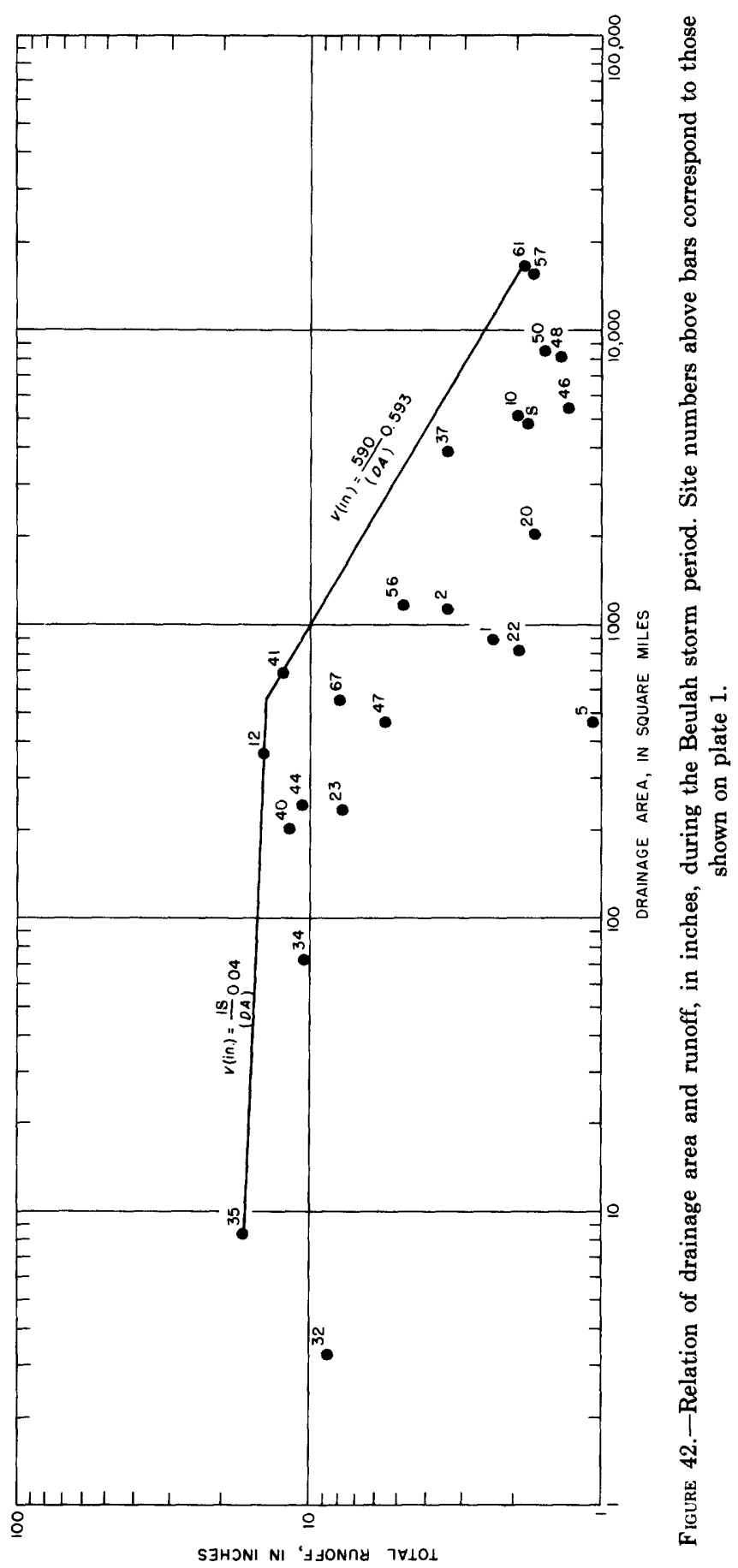




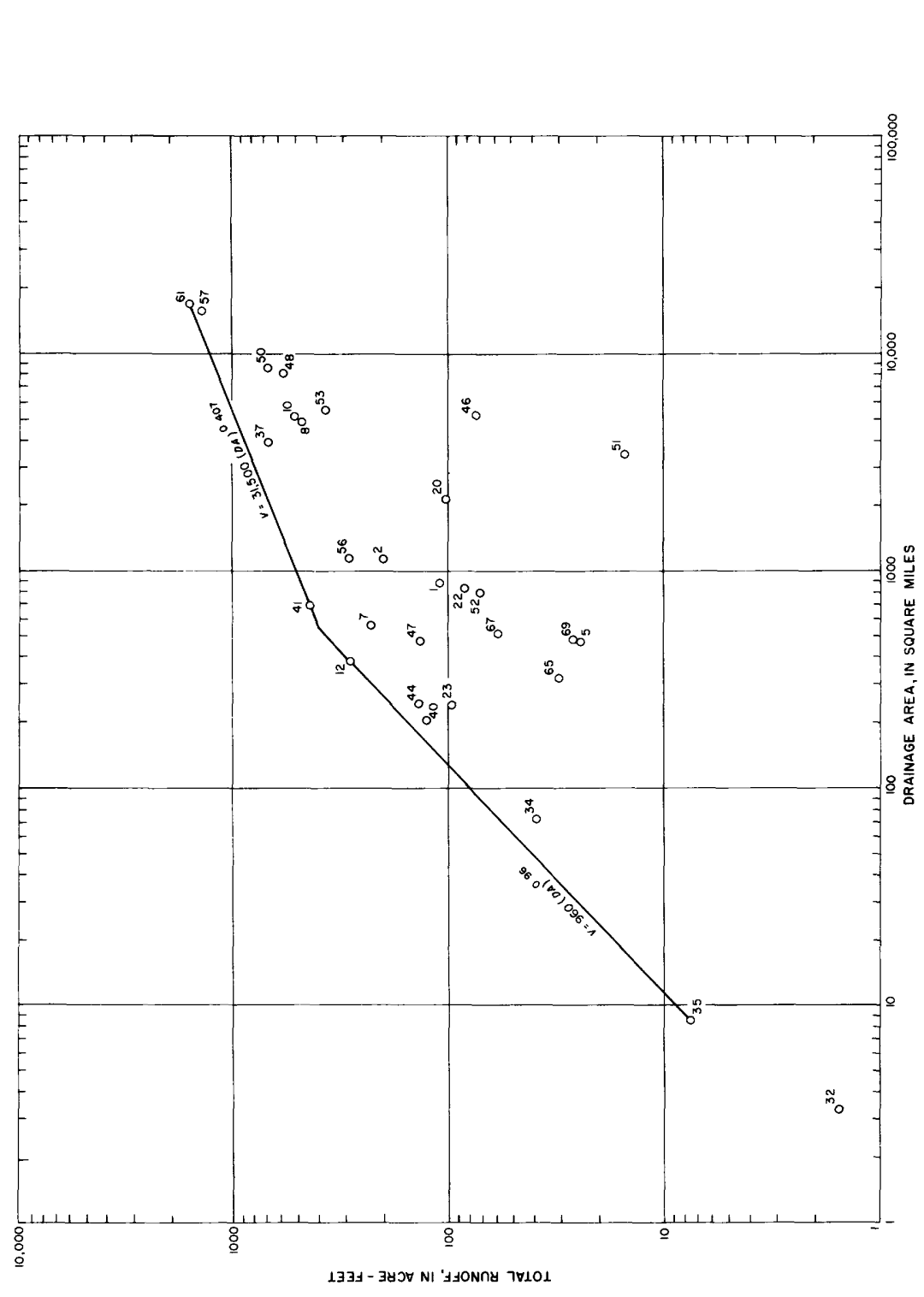

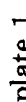

gี

空

旁 웅

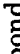

递 


\section{SUMMARY OF FLOOD DAMAGE}

A comprehensive damage survey was conducted by the U.S. Army Corps of Engineers District, Galveston, Tex., after the hurricane subsided. The results of this survey are contained in a flood report prepared by that agency. A summary of these results are cited below.

The expanse of devastation in Beulah's wake covered 39 counties. For the purposes of this report, the portion of the area sustaining most of the damage from Hurricane Beulah on the United States' side of the Rio Grande and in Texas was divided into smaller parts to expedite the post hurricane survey activities. These areas, numbered 1 through 4 , are shown in figure 44 . Wind damages are based on information from the American Insurance Association, insurance adjusters, and insurance companies. Much of the information on the agricultural damages was obtained from the Agricultural Stabilization and Conservation Service and the Soil Conservation Service to the U.S. Department of Agriculture, and county agricultural agents throughout the stricken area.

Table 6 shows the damages from all causes by "damage areas" distributed by classes of property. The property classifications in table 6 are self-explanatory in the cases of residential and commercial properties. The agricultural damage classification includes crop losses, damage to cropland, loss of pastures and the cost of their rehabilitation, damage to irrigation levees and ditches, and the loss of fences, livestock, farm buildings, and equipment. The "other" damage classification includes transportation losses, such as losses to railroads, highways, bridges, vessels, barges, and air facilities; utility losses by power, telephone, telegraph, gas, water, and sewage treatment com-

TABLE 6.-Summary of hurricane damages, by areas, in south-central Texas

\begin{tabular}{|c|c|}
\hline Area 1 & Area 3 \\
\hline $\begin{array}{l}\text { Residential } \ldots \ldots \ldots \ldots \ldots . \$ 33,960,000 \\
\text { Commercial } \ldots \ldots \ldots \ldots \ldots \quad 3,839,000 \\
\text { Agricultural } \ldots \ldots \ldots \ldots \ldots \quad 18,637,000 \\
\text { Other } \ldots \ldots \ldots \ldots \ldots \ldots 25,729,000 \\
\quad \text { Total } \ldots \ldots \ldots \ldots \ldots \$ 82,165,000\end{array}$ & 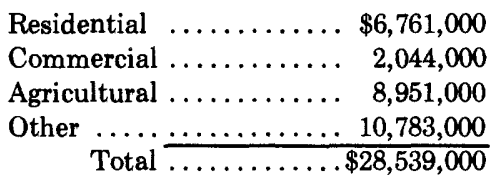 \\
\hline Area 2 & Area 4 \\
\hline 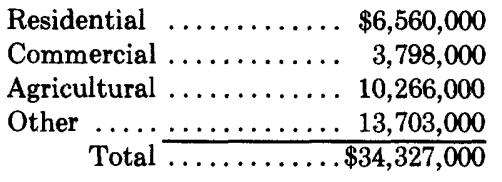 & $\begin{array}{rrr}\text { Residential } \ldots \ldots \ldots \ldots . & \$ 254,000 \\
\text { Commercial } \ldots \ldots \ldots \ldots \ldots & 122,000 \\
\text { Agricultural } \ldots \ldots \ldots \ldots \ldots & \ldots \ldots \ldots \ldots \\
\text { Other } \ldots \ldots & \ldots \ldots \ldots \ldots & 137,000 \\
& \text { Total } \ldots \ldots \ldots \ldots & \$ 513,000\end{array}$ \\
\hline
\end{tabular}

Total damages for areas $1,2,3$, and $4 \ldots \ldots \ldots \ldots \ldots \ldots 145,544,000$

Total damages for areas $1,2,3$, and 4 plus 10 percent .... 160,098,000 




Figure 44.-Damage-survey index map.

panies; and miscellaneous losses, including damages to wharves, docks, piers, public property, drainage canals, navigation channels, levees, churches, and schools. An additional 10 percent was added to total losses as an estimate of less tangible expenses, such as costs arising from plant shutdown, debris removal, combating insects and disease, policing to prevent looting, and business losses.

Damages sustained by selected cities in the four damage areas are tabulated in table 7. The damages to these cities are arranged by primary cause of loss, which includes flooding from tidal overflow, wind and wind-driven rain, and flooding from stream overflow and inadequate drainage. Estimates of damages as "flooding from tidal overflow" include such causes as devastation from high tides, heavy waves, or a combination of these forces. In a similar manner, "wind and winddriven rain" tabulations include all destruction to property from hurricane winds and airborne debris, from tornadoes, and from rain damage through wind-created openings in buildings. "Flooding from stream and inadequate drainage" tabulations include damages caused by stream and drainage-system overflow, surface runoff, and ponded water. 
TABLE 7.-Summary of damages in thousands of dollars, at selected Texas cities affected by Hurricane Beulah

[Based on damage estimates compiled by the U.S. Army Corps of Engineers- - - appear in columns where the total dollar estimates of damages are small. From report on "Hurricane Beulah." 8-21 September 1967, U.S. Army Engineer District, Corps of Engineers, Galveston, Tex., September 1968]

\begin{tabular}{|c|c|c|c|c|}
\hline City & $\begin{array}{l}\text { Flooding from } \\
\text { tidal overflow }^{1}\end{array}$ & $\begin{array}{l}\text { Wind and wind- } \\
\text { driven rain }\end{array}$ & $\begin{array}{c}\text { Flooding from } \\
\text { stream and inadequate } \\
\text { drainage }{ }^{3}\end{array}$ & Total \\
\hline \multicolumn{5}{|c|}{ Area 1} \\
\hline$\ldots \ldots \ldots \ldots \ldots$ & $\ldots$ & 54 & 83 & 137 \\
\hline Arroyo City . . . . . . . . . . . . & $\ldots$ & 68 & 127 & 195 \\
\hline Brownsville $\ldots \ldots \ldots \ldots \ldots$ & $\ddot{23}$ & 835 & 327 & 1,185 \\
\hline Edinburg $\ldots \ldots \ldots \ldots \ldots \ldots$ & & 556 & 2,661 & 3,217 \\
\hline Harlingen $\ldots \ldots \ldots \ldots \ldots$ & & 445 & 3,203 & 3,648 \\
\hline La Grulla $\ldots \ldots \ldots \ldots \ldots \ldots$ & & 73 & 200 & 273 \\
\hline Laguna Vista . . . . . . . . . . & $\ddot{3}$ & 150 & & 153 \\
\hline Lyford $\ldots \ldots \ldots \ldots \ldots \ldots$ & $\cdots$ & $\because$ & 85 & 85 \\
\hline McAllen ..... & . & 369 & 915 & 1,284 \\
\hline Port Isabel & 7 & 3,000 & 2 & 3,009 \\
\hline Port Mansfield ....... & 22 & 500 & $2 \overline{5}$ & 547 \\
\hline Raymondville ........... & $\cdots$ & 231 & 732 & 963 \\
\hline Rio Grande City ........... & & 44 & 970 & 1,014 \\
\hline San Juan ............... & & 64 & 159 & 223 \\
\hline San Perlita & & 36 & 177 & 213 \\
\hline
\end{tabular}

Area 2

\begin{tabular}{|c|c|c|c|c|}
\hline Aransas Pass ..... & 1 & 34 & 759 & 794 \\
\hline 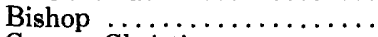 & & 150 & 142 & 292 \\
\hline Corpus Christi .......... & 2,197 & 106 & 472 & 2,775 \\
\hline Driscoll . . . . . . . . . . . . & & 9 & 162 & 171 \\
\hline 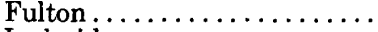 & 287 & 1,166 & & 1,453 \\
\hline Ingleside $\ldots \ldots \ldots \ldots \ldots \ldots$ & . & 67 & 164 & 231 \\
\hline Kingsville $\ldots \ldots \ldots \ldots \ldots$ & & 27 & 396 & 423 \\
\hline 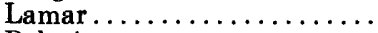 & 53 & 18 & 27 & 9 \\
\hline Palacios $\ldots \ldots \ldots \ldots \ldots \ldots$ & $\cdots$ & 214 & 27 & 241 \\
\hline Port Aransas $\ldots \ldots \ldots \ldots \ldots$ & 28 & 63 & $\cdots$ & 9 \\
\hline Port Lavaca . . . . . . . . . . . . & 71 & 93 & 11 & 17 \\
\hline Robstown $\ldots \ldots \ldots \ldots \ldots \ldots$ & & 14 & 423 & 437 \\
\hline Rockport $\ldots \ldots \ldots \ldots \ldots \ldots$ & 313 & 8 & 676 & 997 \\
\hline Sinton $\ldots \ldots \ldots \ldots \ldots \ldots$ & $\cdots$ & 48 & 2,690 & 2,73 \\
\hline
\end{tabular}

Area 3

\begin{tabular}{|c|c|c|c|c|}
\hline Alice & ... & 150 & 97 & 247 \\
\hline Beeville $\ldots \ldots \ldots \ldots \ldots \ldots$. & . . & 50 & 157 & 207 \\
\hline Cuero ..... & . . & 8 & 182 & 190 \\
\hline Falfurrias ......... & $\ldots$ & 98 & 3,310 & 3,408 \\
\hline Goliad ........ & $\ldots$ & 36 & 95 & 131 \\
\hline Hebbronville ......... & $\cdots$ & 83 & 46 & 129 \\
\hline Kenedy ............. & . . & 4 & 242 & 246 \\
\hline Pettus . . . . . . . . . . . & . . & 10 & 163 & 173 \\
\hline Refugio ................. & . . & 36 & 170 & 206 \\
\hline Three Rivers . . . . . . . . . & . & 75 & 2,649 & 2,724 \\
\hline Victoria $\ldots \ldots \ldots \ldots \ldots \ldots$ & . . & 56 & 282 & 338 \\
\hline Yorktown ............. & $\ldots$ & 33 & 790 & 823 \\
\hline
\end{tabular}

Area 4

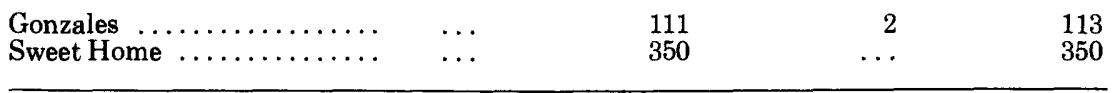

\footnotetext{
${ }^{1}$ Includes such damages from high tides, wave action, or a combination of these destructive agents.

${ }^{2}$ Includes such damages to structures as direct wind blasts of hurricane winds, tornadoes, airborne debris smashing into roofs and walls, or from rain damage through wind-created openings in buildings.

${ }^{3}$ Includes such causes as damages to property and life caused by torrential rain runoff being so great that stream and drainage systems overflowed onto adjacent areas.
} 
The total damage from all causes in the areas stricken by Hurricane Beulah was estimated at $\$ 168,844,000$. The total damages from hurricane tidal overflow in areas 1,2 , and 3 were estimated to be $\$ 5$,449,000; the total damages caused by wind and wind-driven rain in all four damage areas were estimated to be $\$ 46,491,000$; the total flood damages from stream overflow and inadequate drainage were estimated to be $\$ 108,158,000$. Other estimated damages caused by Hurricane Beulah include $\$ 5,461,000$ expended by the Red Cross and other disaster relief agencies, $\$ 800,000$ expended by the Texas State Department of Health, $\$ 28,000$ expended by the Department of the Army in "Operation Bravo," and $\$ 2,457,000$ expended by the U.S. Army Corps of Engineers for added maintenance to river and harbor facilities damaged by Hurricane Beulah. A summary of the real property damages, including those damages which could not be separated by areas, is given in table 6.

According to statistics compiled by the U.S. Weather Bureau, at least 57 deaths were attributed to Hurricane Beulah. These deaths were reported as follows: Martinique, 15; St. Vincent, 2; Puerto Rico, 1; Dominican Republic, 2; Yucatan, 5; Northern Mexico, 19; and Texas, 13. In Texas, 5 of the deaths were caused by tornadoes and 8 by drowning. An additional 37 persons were reported injured, with 34 of these being injured in tornadoes. The combination of early warning and mass evacuation of the population from the storm path greatly reduced the number of probable casualties.

\section{RESULTS OF FRESHWATER INFLOW TO BAYS AND ESTUARIES}

By D. C. $\mathrm{H}_{\mathrm{AHL}}$

The floods caused by Hurricane Beulah presented a unique opportunity to study the effects of a large volume of freshwater inflow to bays and estuaries along the Texas coast. Nueces-Corpus Christi and Guadalupe-San Antonio Bays were selected for study because of their accessibility and proximity to populated areas and because of their differences in configuration, surface area, and depth.

Data were collected on both bay systems and their tributary streams along traverses. Verticals were selected at points $1 / 2-1$ mile apart except where changes in water quality indicated that other spacing was more suitable. Channels were sampled wherever crossed by the lines of traverse.

Dissolved oxygen, specific conductance, and temperature measurements were made from the water surface to the bottom at most data-collection points. Samples for laboratory analysis were taken at selected points. In addition to the major chemical constituents determined in the laboratory, strontium $(\mathrm{Sr})$, lithium $(\mathrm{Li})$, nitrate $\left(\mathrm{NO}_{3}\right)$, 
TABLE 8.-Suspended-sediment concentrations, in milligrams per liter, in the Nueces River and Corpus Christi Bay, September 27-29, 1967

\begin{tabular}{|c|c|c|c|}
\hline & $\begin{array}{l}\text { September } \\
\quad 27\end{array}$ & $\begin{array}{l}\text { September } \\
\quad 28\end{array}$ & $\begin{array}{c}\text { September } \\
29\end{array}$ \\
\hline Nueces River near Mathis ...... & & 156 & \\
\hline $\begin{array}{l}\text { Nueces River at U.S. Highway } 77 \text {, } \\
\text { near Calallen } \ldots \ldots \ldots \ldots \ldots \ldots \ldots\end{array}$ & 374 & 262 & \\
\hline $\begin{array}{l}\text { Cross section } C-C \text {, Corpus } \\
\text { Christi Bay at U.S. Highway } \\
\text { 181, at Corpus Christi } \ldots \ldots \ldots\end{array}$ & $\ldots$ &. & 76 \\
\hline
\end{tabular}
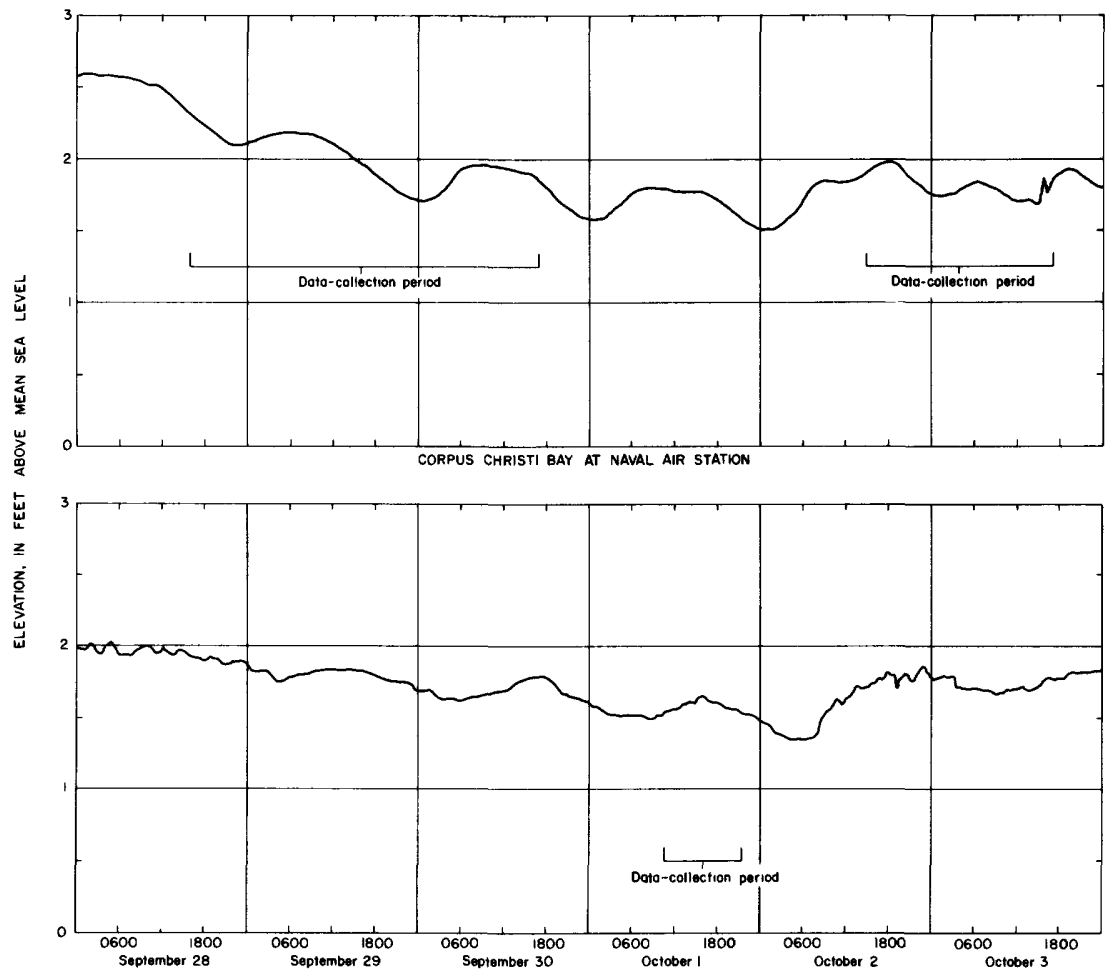

SAN ANTONIO BAY AT VICTORIA CHANNEL

FiguRE 45.-Periods of data collection and stages of Corpus Christi and San Antonio Bays, September 28-October 3, 1967.

and phosphate $\left(\mathrm{PO}_{4}\right)$ were determined for a few samples. Suspendedsediment concentrations in the Nueces River and Corpus Christi Bay are given in table 8 . The results of field measurements are given in tables 10,12, and 14; results of laboratory analyses are given in table 9 , 11, and 13. The periods of data collection and stages of Corpus Christi and San Antonio Bays are shown in figure 45.

Figure 46 shows the relation of specific conductance to concentrations of dissolved solids and chloride. 


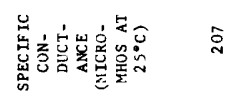

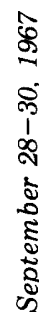

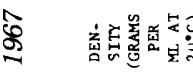

1े

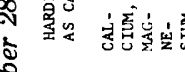

岑

思悹

总

हें

$\frac{\sqrt{2}}{\sqrt[5]{5}}$

告

.5

용

के

运

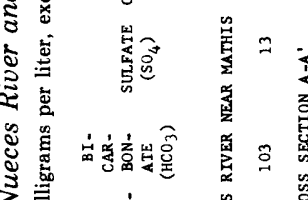

宫寅画

它崖产

亨崫的

崖

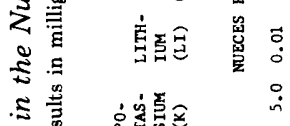

.5

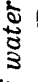

$$
\text { 密 }
$$

递密黄

客安总

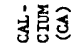

葛氮

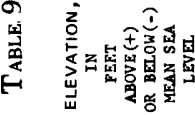

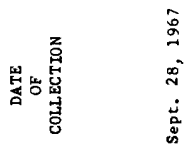

ฐ ฐ

00

$\stackrel{+\infty}{\infty}$

$\operatorname{4ลำ~}$

$\stackrel{0}{0} \quad \stackrel{0}{0} \stackrel{0}{0}$

웅

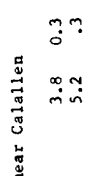

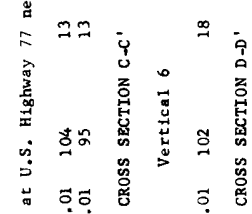

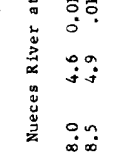

:̊ํ:

$\stackrel{0}{\stackrel{4}{i}}$

$\vec{m}$ 옹

$\dot{a}=$

ఏฏ

के

ì ำing

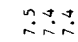

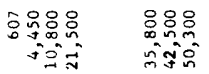

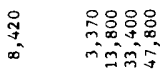

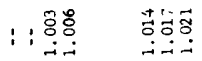

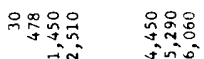

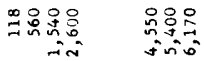

品

旁: : : : :

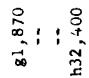

$\stackrel{\infty}{3}: 1: \quad: 1: \frac{1}{3}$

苟:

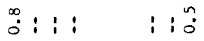

m: : : i:

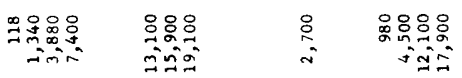

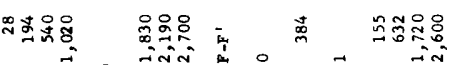

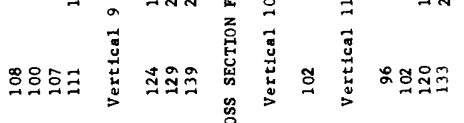

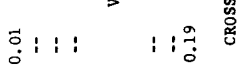

赫: : i i

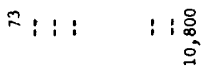

กิ $1:$ :

$\stackrel{0}{0}: 1: 1: 1: \frac{1}{0}$

$\stackrel{0}{9}:: \frac{0}{0}$

$\stackrel{5}{\infty}$

a: : : : : : 娄

ก: : : :

की $1:$ :

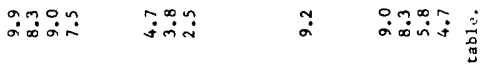

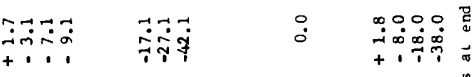

ま

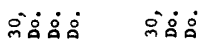

๓ั่

ڤัँ

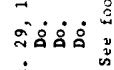




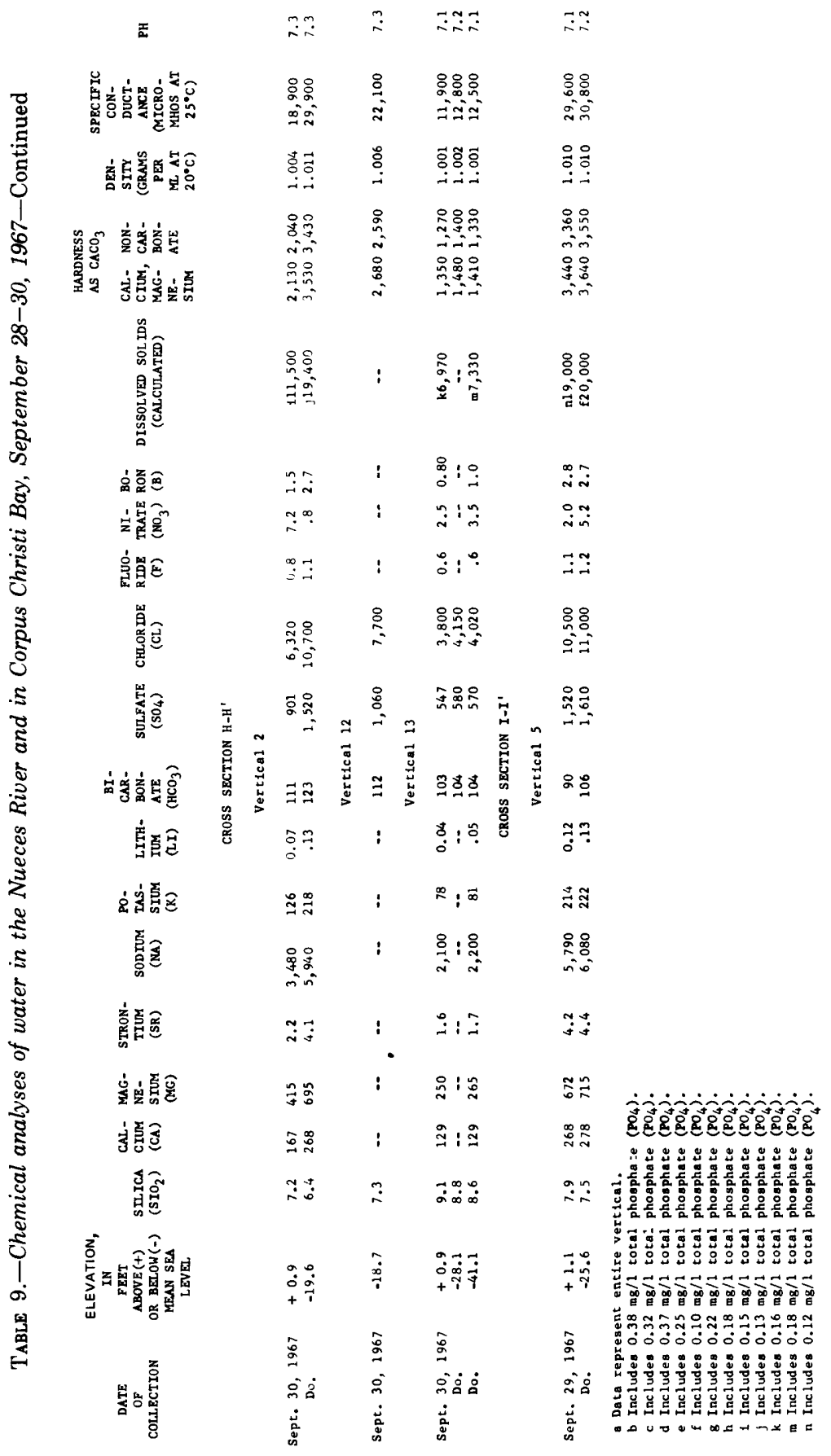


TABLE 10.-Field determinations of water-quality data for Corpus Christi Bay, September

$$
28-30,1967
$$

$\begin{array}{lccc}\text { ELEVATION, } & \text { SPEC!FIC } & \text { DISSOLVED } \\ \text { FEET } & \text { TEMPER- } & \begin{array}{c}\text { CONDUCT- } \\ \text { OXYGEN }\end{array} \\ \text { ABOVE(+) } & \text { ATURE } & \text { ANCE } & \text { (MILLI- } \\ \text { OR BELOW(-) } & \left({ }^{\circ} \mathrm{C}\right) & \text { (MICRO- } & \text { GRAMS PER } \\ \text { MEAN SEA } & & \text { MHOS AT } & \text { LITER) } \\ \text { LEVEL } & & \left.25^{\circ} \mathrm{C}\right) & \end{array}$

Cross Section C-C' - September 28, 1967a/

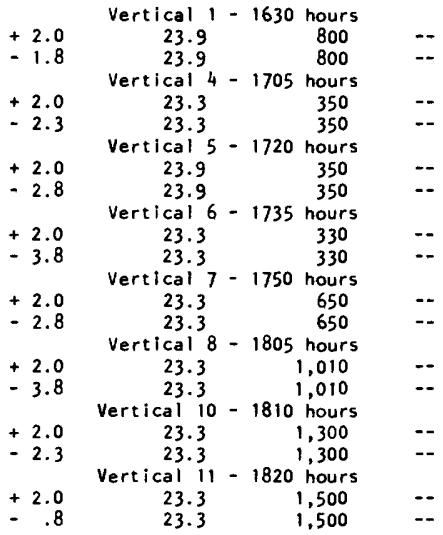

Cross Section 0-D' - September 30, 1967b/

\begin{tabular}{cccc}
\multicolumn{4}{c}{ Vertical 1 - 1050 hours } \\
+1.8 & 23.5 & 9,200 & 6.3 \\
-5.5 & 23.5 & 9,600 & 6.3 \\
& Vertical $2-1110$ hours \\
+1.8 & 24.0 & 8,300 & 6.0 \\
-3.5 & 24.0 & 9,600 & 6.0 \\
-6.5 & 24.0 & 11,600 & 5.9 \\
-9.5 & 24.0 & 13,000 & 5.8 \\
-12.5 & 25.0 & 37,300 & 4.0 \\
+1.8 & Vertical 3- 1130 hours \\
-1.5 & 24.0 & 7,700 & 5.5 \\
-3.5 & 24.0 & 10,100 & 5.5 \\
-5.5 & 24.0 & 12,100 & 5.4 \\
-7.5 & 24.0 & 12,100 & 5.4 \\
-9.5 & 24.0 & 14,600 & 5.4 \\
-11.5 & 24.3 & 14,600 & 5.3 \\
-13.5 & 25.5 & 30,500 & 4.4 \\
+1.8 & 25.5 & 39,200 & 3.9 \\
-3.5 & Vertical 4 & 1200 hours & \\
-5.5 & 24.0 & 2,900 & 5.5 \\
-7.5 & 24.0 & 5,700 & 5.4 \\
-9.5 & 24.0 & 8,800 & 5.4 \\
-11.5 & 24.0 & 11,200 & 5.3 \\
-13.5 & 24.0 & 11,600 & 5.2 \\
& 25.0 & 19,500 & 4.8 \\
& 25.5 & 35,400 & 4.2
\end{tabular}

See footnotes at end of table.

$\begin{array}{cccc}\begin{array}{c}\text { ELEVATION, } \\ \text { FEET }\end{array} & \text { TEMPER- } & \begin{array}{c}\text { SPECIFIC } \\ \text { CONDUCT- }\end{array} & \begin{array}{c}\text { DISSOLVED } \\ \text { OXYGEN }\end{array} \\ \text { ABOVE (+) } & \text { ATURE } & \text { ANCE } & \text { (MILLI- } \\ \text { OR BELOW(-) } & \left({ }^{\circ} \mathrm{C}\right) & (\text { MICRO- } & \text { GRAMS PER } \\ \text { MEAN SEA } & & \text { MHOS AT } & \text { LITER) } \\ \text { LEVEL } & & \left.25^{\circ} \mathrm{C}\right) & \end{array}$

Cross Section $0-D^{\prime}$

September $30,1967 \mathrm{~b} /--$ Continued

$+1.8$

Vertical 5 - 1225 hours

$-3.0$

- 5.0

$-7.0$

- 9.0

$-11.0$

$-13.0$

$+1.7$

$-3.1$

$-5.1$

- 7.1

$-9.1$

$-11.1$

$-13.1$

1.7
-1.1

$-1.1$

- 3.6

- 5.6

- 7.6

$-9.6$

$-11.6$

$+1.7$

$-1.1$

- 3.1

- 5.1

$-7.1$

$-9.1$

$-11.1$

$+1.7$

- 2.1

$-7.1$

$-12.1$

$-17.1$

$-22.1$

$-27.1$

$-32.1$

$-37.1$

$-42.1$

$+1.7$

- 1.1

- 3.1

- 5.1

- 7.1

- 9.1

-11.1
-13.1

$\begin{array}{ll}25.5 & 3,100 \\ 24.5 & 5,800 \\ 24.5 & 9,200 \\ 24.5 & 11,200 \\ 25.0 & 15,600 \\ 25.5 & 29,500 \\ 26.0 & 39,200\end{array}$

Vertical 6 - 1300 hours $25.0 \quad 2,900$

25.0

25.0

25.0

$26.0 \quad 29,500$

Vertical 7 - 1330 hours $25.0 \quad 1,250$

25.0

1,250

$25.0 \quad 7,200$

$25.0 \quad 9,700$

$25.0 \quad 13,600$

$25.0 \quad 17,600$

$25.0 \quad 33,500$

Vertical 8 - 1355 hours

$26.0 \quad 680$

$23.5 \quad 3.400$

$24.0 \quad 4,800$

$25.0 \quad 8,800$

$25.0 \quad 11,800$

$25.5 \quad 22,500$

$26.0 \quad 33,500$

Vertical 9 - 1440 hours

$\begin{array}{lll}26.0 & 1,600 & 4.9\end{array}$

$24.5 \quad 3,200 \quad 5.2$

$25.0 \quad 12,100 \quad 5.0$

$\begin{array}{lll}25.5 & 30,500 & 4.8\end{array}$

$\begin{array}{lll}26.0 & 33,500 & 4.6\end{array}$

$26.5 \quad 39,200 \quad 4.2$

$27.0 \quad 39,200 \quad 3.9$

$27.0 \quad 44,100 \quad 3.4$

$27.0 \quad 48,800$

$27.0 \quad 48,800 \quad 3.0$

Vertical 10 - 1515 hours

$\begin{array}{lll}26.0 & 3,500 & 4.6\end{array}$

$\begin{array}{lll}25.0 & 4.500 & 4.8\end{array}$

$25.0 \quad 4,500 \quad 4.6$

$24.0 \quad 8,300 \quad 5.4$

$24.0 \quad 14,600 \quad 5.3$

$\begin{array}{lll}24.5 & 19,500 & 5.2\end{array}$

$25.0 \quad 27,400 \quad 5.0$

$\begin{array}{lll}26.0 & 31,000 & 4.8\end{array}$

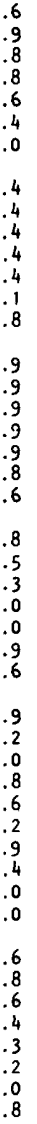


TABLE 10.-Field determinations of water-quality data for Corpus Christi Bay, September 28-30, 1967-Continued

\begin{tabular}{|c|c|c|c|}
\hline $\begin{array}{l}\text { ELEVATION, } \\
\text { FEET } \\
\text { ABOVE (+) } \\
\text { OR BELOW(-) } \\
\text { MEAN SEA }\end{array}$ & $\begin{array}{l}\text { TEMPER- } \\
\text { ATURE } \\
\left({ }^{\circ} \mathrm{C}\right)\end{array}$ & $\begin{array}{l}\text { SPECIFIC } \\
\text { CONDUCT- } \\
\text { ANCE } \\
\text { (MICRO- } \\
\text { MHOS AT } \\
25^{\circ} \mathrm{C} \text { ) }\end{array}$ & $\begin{array}{l}\text { DISSOLVED } \\
\text { OXYGEN } \\
\text { (MILLI- } \\
\text { GRAMS PER } \\
\text { LITER) }\end{array}$ \\
\hline
\end{tabular}

Cross Section D-D '

September 30, 1967b/--Cont inued

\begin{tabular}{cccc} 
& Vertical $11-1535$ hours \\
+1.7 & 26.0 & 3,000 & 4.6 \\
-.6 & 24.5 & 6,300 & 5.0 \\
-2.6 & 24.5 & 9,700 & 5.0 \\
-4.6 & 24.5 & 12,600 & 5.0 \\
-6.6 & 24.5 & 13,600 & 5.0 \\
-8.6 & 25.0 & 25,500 & 4.8 \\
-10.6 & 26.0 & 31,400 & 4.3 \\
-12.6 & 26.5 & 44,100 & 3.7 \\
+1.7 & Vertical $12-1600$ hours \\
+1.7 & 25.5 & 4,300 & 4.9 \\
-2.6 & 24.5 & 8,800 & 5.2 \\
-4.6 & 24.5 & 10,700 & 5.2 \\
-6.6 & 24.5 & 12,800 & 5.2 \\
-8.6 & 25.0 & 27,500 & 5.0 \\
-10.6 & 26.0 & 33,500 & 4.8 \\
-12.6 & 27.0 & 44,100 & 2.6 \\
& Vertical $13-1625$ hours \\
+1.7 & 25.0 & 5,000 & 5.0 \\
-1.7 & 25.0 & 5,000 & 5.0 \\
-2.1 & 24.5 & 5,700 & 5.0 \\
-2.1 & 24.0 & 8,800 & 5.1 \\
-4.1 & 24.0 & 10,700 & 5.1 \\
-6.1 & 24.5 & 19,500 & 4.8 \\
-8.1 & 25.0 & 26,000 & 4.7 \\
-10.1 & 26.5 & 44,100 & 3.7 \\
-12.1 & Vertical $14-1705$ hours \\
+1.6 & 24.5 & 4,800 & 4.3 \\
+1.6 & 24.5 & 4,800 & 4.3 \\
-2.2 & 24.0 & 4,800 & 4.8 \\
-4.2 & 24.0 & 4,900 & 4.8 \\
-6.2 & 24.0 & 12,100 & 4.6 \\
-8.2 & 25.5 & 39,200 & 4.0 \\
-10.2 & \multicolumn{4}{c}{}
\end{tabular}

Cross Section E-E' - September 30, 1967 b/

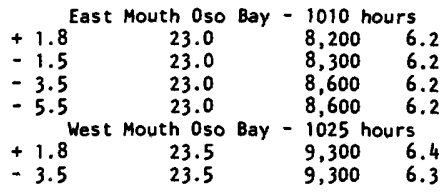

Cross Section F-F' - September 29, 1967\&

$\begin{array}{rrrr} & \text { Vertical 1 } & 1040 \text { hours } \\ +1.9 & 23.0 & 9,400 & 8.0 \\ +.1 & 23.0 & 9,700 & 8.0 \\ -1.9 & 23.5 & 10,700 & 7.9 \\ -3.9 & 23.5 & 11,200 & 7.9 \\ -5.9 & 23.5 & 13,600 & 7.9 \\ -8.9 & 23.0 & 13,600 & 8.0\end{array}$

See footnotes at end of table.

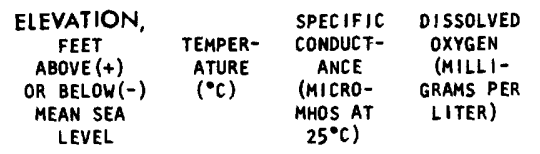

Cross Section F-F'

September 29, 1967 $\subseteq /-$-Conti inued

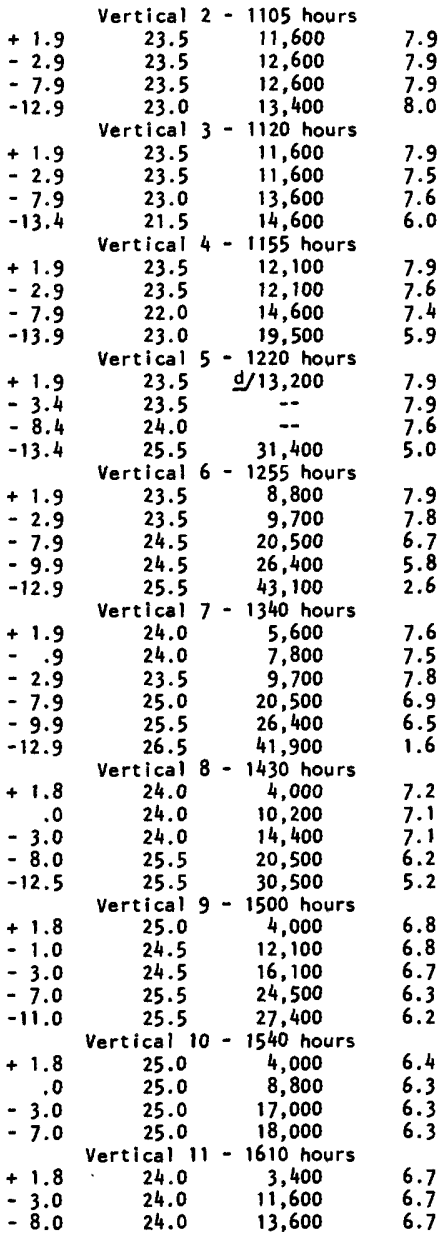


TABLE 10.-Field determinations of water-quality data for Corpus Christi Bay, September 28-30, 1967-Continued

$\begin{array}{cccc}\begin{array}{c}\text { ELEVATION, } \\ \text { FEET }\end{array} & \text { TEMPER- } & \begin{array}{c}\text { SPECIFIC } \\ \text { CONDUCT- }\end{array} & \begin{array}{c}\text { DISSOLVED } \\ \text { OXYGEN }\end{array} \\ \text { ABOVE }(+) & \text { ATURE } & \text { ANCE } & \text { (MILLI- } \\ \text { OR BELOW(-) } & \left({ }^{\circ} \mathrm{C}\right) & \text { (MICRO- } & \text { GRAMS PER } \\ \text { MEAN SEA } & & \text { MHOS AT } & \text { LITER) } \\ \text { LEVEL } & & \left.25^{\circ} \mathrm{C}\right) & \end{array}$

Cross Section F-FI

September 29, 1967c/--Continued

\begin{tabular}{cccc} 
Vertical & $11-$ & \multicolumn{3}{c}{1610} & hours--Cont inued \\
-13.0 & 25.0 & 27,400 & 6.0 \\
-18.0 & 26.0 & 33,500 & 5.1 \\
-23.0 & 25.5 & 39,300 & 4.2 \\
-28.0 & 26.5 & 44,100 & 3.4 \\
-33.0 & 26.5 & 46,900 & 3.4 \\
-38.0 & 26.5 & 46,900 & 3.2
\end{tabular}

Cross Section G-G' - September 19, 1967

$\begin{array}{rrrr} & \text { Vertical 1 - } 1330 \text { hours } \\ +1.9 & 21.7 & 16,700 & 12.6 \\ -1.5 & 21.7 & 16,700 & 12.6 \\ +1.9 & \text { Vertical 2 - } 1400 \text { hours } \\ +1.5 & 22.2 & 14,600 & 10.7 \\ & 22.2 & 14,600 & 10.7 \\ +1.0 & \text { Vertical 3 } 31500 \text { hours } \\ -8.0 & 22.2 & 25,800 & 12.1 \\ -15.5 & 21.7 & 30,000 & 10.4 \\ & 22.2 & 30,000 & 10.0 \\ +1.0 & \text { Vertical 4 - 1530 hours } \\ -3.5 & 22.2 & 29,000 & 10.8 \\ & 22.2 & 29,000 & 10.8\end{array}$

Cross Section $\mathrm{H}^{-H^{\prime}}$ - September 30, 1967

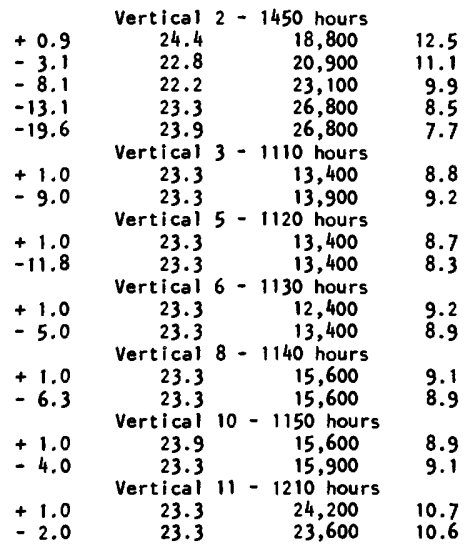

$\begin{array}{cccc}\text { ELEVATION, } & & \text { SPECIFIC } & \text { DISSOLVED } \\ \text { FEET } & \text { TEMPER- } & \text { CONDUCT- } & \text { OXYGEN } \\ \text { ABOVE(+) } & \text { ATURE } & \text { ANCE } & \text { (MILLI- } \\ \text { OR BELOW(-) } & \text { ('C) } & \text { (MICRO- } & \text { GRAMS PER } \\ \text { MEAN SEA } & & \text { MHOS AT } & \text { LITER) } \\ \text { LEVEL } & & 25^{\circ} \mathrm{C} \text { ) } & \end{array}$

Cross Section $\mathrm{H}^{-\mathrm{H}^{\prime}}$ September $30,1967 \mathrm{~b} /-$-Cont inued

$\begin{array}{cccc} & \text { Vertical } 12-1235 \text { hours } \\ +0.9 & 23.3 & 11,800 & 8.5 \\ -8.1 & 23.3 & 12,900 & 8.2 \\ -13.1 & 23.3 & 12,900 & 8.2 \\ -15.1 & 23.3 & 13,400 & 8.0 \\ -16.1 & 23.3 & 13,900 & 7.8 \\ -18.7 & 23.9 & 19,800 & 7.1 \\ & \text { Verticai } 13-1320 \text { hours } & \\ +0.9 & 23.3 & 12,400 & 8.6 \\ -8.1 & 23.3 & 13,400 & 8.7 \\ -18.1 & 23.3 & 18,800 & 7.6 \\ -28.1 & 23.9 & \text { d } 12,800 & -. \\ -41.1 & 23.3 & d / 12,500 & -. \\ & \text { Vertical } 14-1305 \text { hours } & \\ +0.9 & 23.3 & 12,900 & 8.8 \\ -8.1 & 23.3 & 13,900 & 9.1 \\ -13.6 & 23.3 & 13,900 & 8.9\end{array}$

Cross Section 1-1' - September 29, 1967 $/$

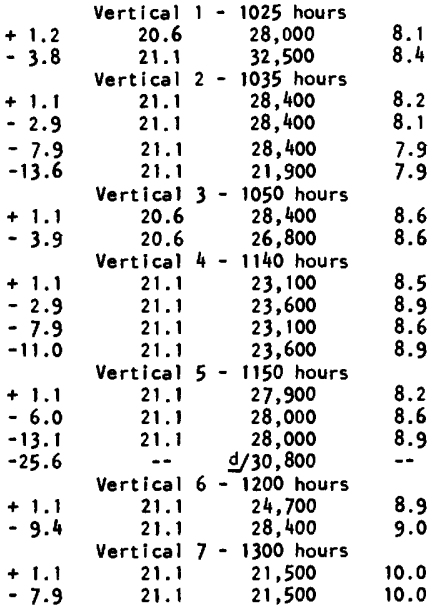

a/ Stage of bay fluctuated from +2.3 feet at 1600 hours to +2.2 feet at 1900 hours. b) Stage of bay fluctuated from +2.0 feet at 1000 hours to +1.8 feet at 1700 hours. c) Stage of bay fluctuated from +2.2 feet at 1000 hours to +2.0 feet at 1600 hours. d) Determined in Laboratory. 
I

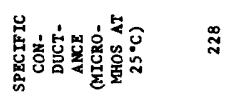

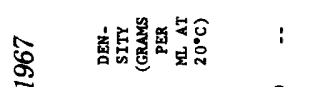

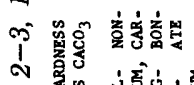

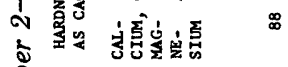

ठ

है

苍是

蒙 苔

है

这 运罢色 กั่อ

害整

芯

ֻัँ

$\cong$

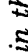

ฐัฐ

o

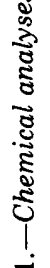

龍部

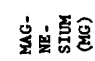

蛋导䓫

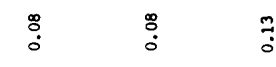

悹总

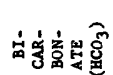

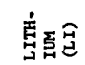

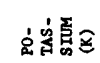

唇产

$\stackrel{-1}{+}$

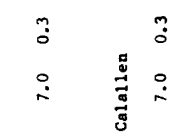

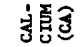

ริำ

望=

量

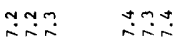

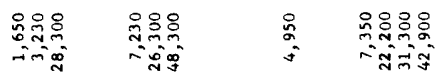

$1:$ :

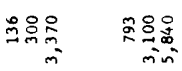

ส๊

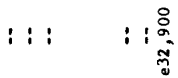

:

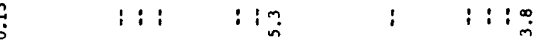

: : : : :

:1: $1: \stackrel{0}{1} \quad: \quad 1: 1:$

สำํำ

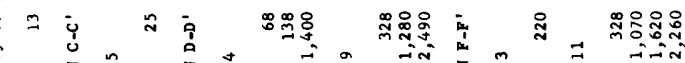

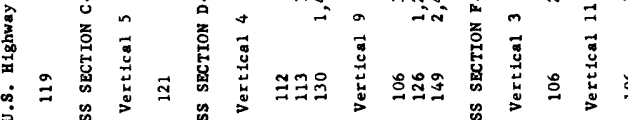

$: 1: \quad: 1 \frac{9}{0}$

$\therefore: \quad: i \approx$

$:: \quad: 1 \frac{0}{0}$

$\therefore: \quad: \because \stackrel{0}{0}$

$:: \quad: 18$

:1: $: 1: \frac{9}{9}$

$==\frac{m}{\infty}$ क्ष

ํำ

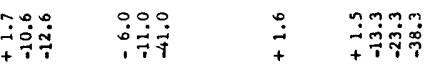

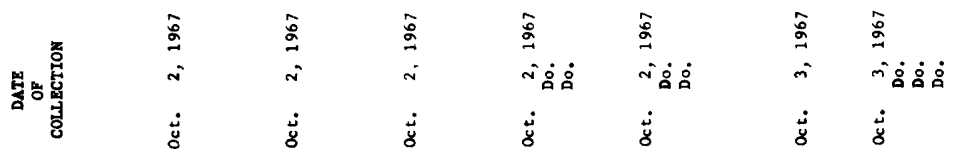


SOUTH TEXAS AND NORTHEASTERN MEXICO, SEPT.-OCT. B75

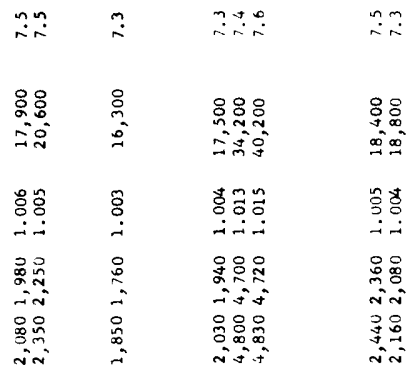

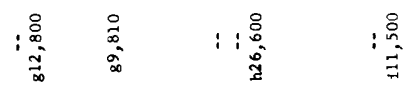

$\stackrel{\infty}{i} \quad \stackrel{i}{i} \quad: \stackrel{n}{i} \quad: \stackrel{n}{i}$

$: \stackrel{\infty}{m} \quad \stackrel{\infty}{m} \quad: 1: \stackrel{\infty}{i} \quad: i$

i⿺

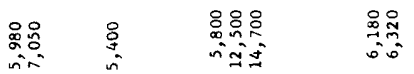

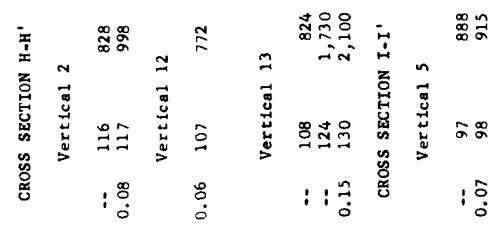

iฐ

离

$\stackrel{\infty}{i} \quad \stackrel{0}{i} \quad: i \stackrel{\infty}{i} \quad: \stackrel{\infty}{i}$

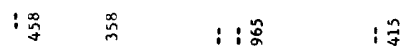

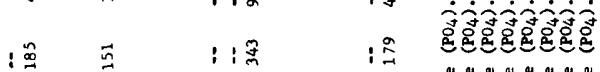

足

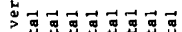

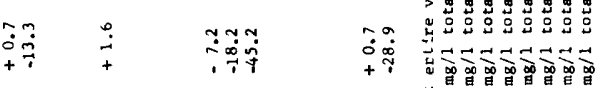

ま ま ま

के

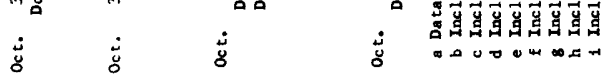


TABLE 12.-Field determinations of water-quality data for Corpus Christi Bay, October $2-3,1967$

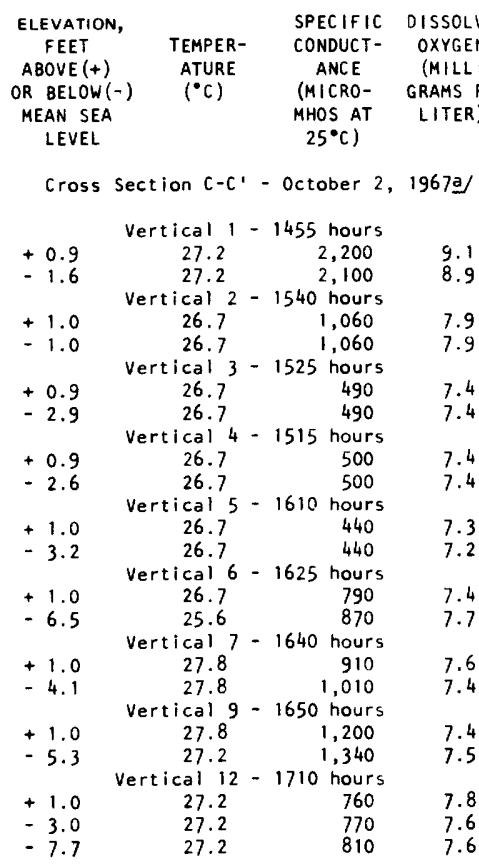

Cross Section D-D' - October 2, 1967a

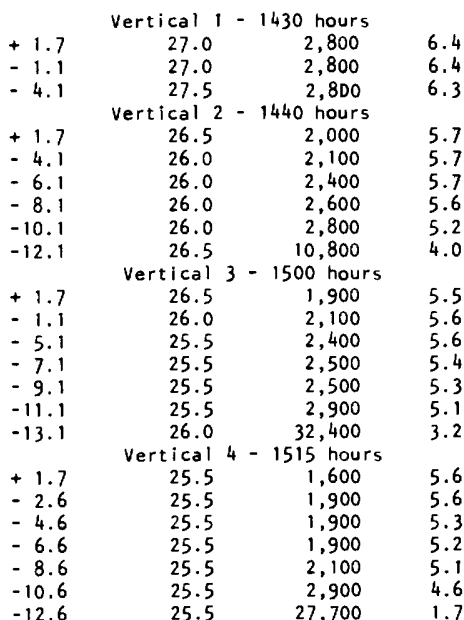

See footnotes at end of table.

$\begin{array}{cccc}\text { ELEVATION, } & & \text { SPECIFIC } & \text { DISSOLVED } \\ \text { FEET } & \text { TEMPER- } & \text { CONDUCT- } & \text { OXYGEN } \\ \text { ABOVE }(+) & \text { ATURE } & \text { ANCE } & \text { (MILLI- } \\ \text { OR BELOW }(-) & \left({ }^{\circ} \mathrm{C}\right) & \text { (MICRO- } & \text { GRAMS PER } \\ \text { MEAN SEA } & & \text { MHOS AT } & \text { LITER) } \\ \text { LEVEL } & & \left.25^{\circ} \mathrm{C}\right) & \end{array}$

Cross Section D-D. October 2, 1967a/--Continued

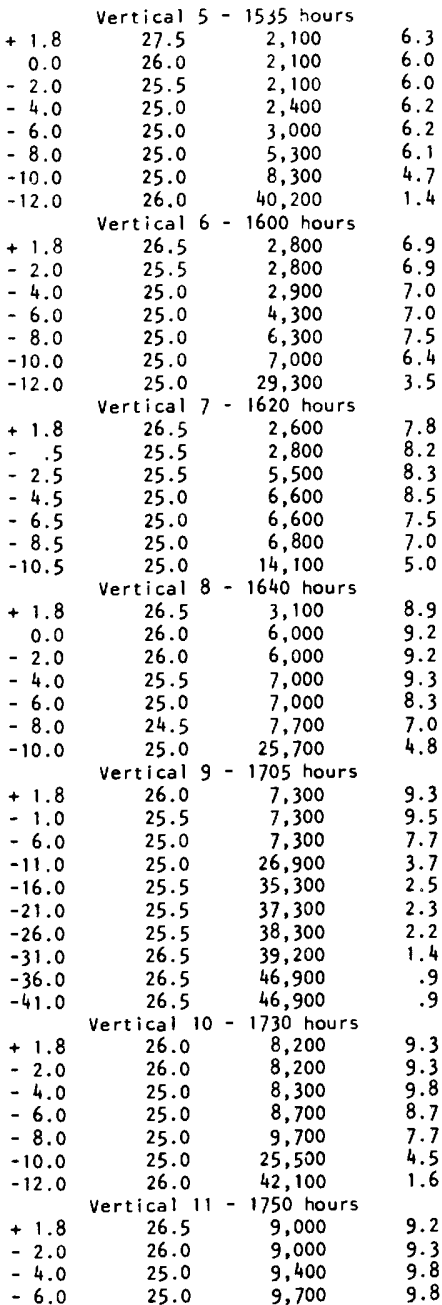


TABLE 12.-Field determinations of water-quality data for Corpus Christi Bay, October 2-3, 1967-Continued

$\begin{array}{cccc}\text { ELEVATION, } & \text { SPECIFIC } & \text { DISSOLVED } \\ \text { FEET } & \text { TEMPER- } & \text { CONDUCT - } & \text { OXYGEN } \\ \text { ABOVE }(+) & \text { ATURE } & \text { ANCE } & \text { (MILLI- } \\ \text { OR BELOW }(-) & \left({ }^{\circ} \mathrm{C}\right) & \text { (MICRO- } & \text { GRAMS PER } \\ \text { MEAN SEA } & & \text { MHOS AT } & \text { LITER) } \\ \text { LEVEL } & & \left.25^{\circ} \mathrm{C}\right) & \end{array}$

Cross Section D-D'

October 2, 1967a/--Cont inued

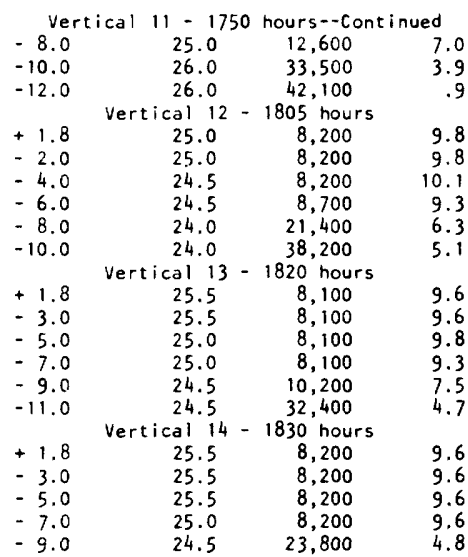

Cross Section F-F' - October 3, 1967b/

$+1.6$

$-1.2$

$-3.2$

$-5.2$

$-7.2$

$-9.2$

$+1.6$

$-.7$

-2.7
$-\quad 4.7$

$-6.7$

$-8.7$

$-10.7$

$-12.7$

$+1.6$

$-1.2$

- 3.2

$-5.2$

- 7.2

$-9.2$

$-13.2$

$+1.6$

$-1.7$

-1.7
-5.7

Vertical 1 - 0920 hours

$$
25.0
$$

9,900

25.0

10,200

11.1

25.5

10,800

11,100

25.0

12,100

Vertical 2 - 0935 hours 25.0 - 5,600

$25.0 \quad 5,600$

25.5

7,300

11,900

14,100

25.0

25.0

25.0

16,600
25,400

41,100

Vertical 3 - 0.955 hours

25.0

5,000

5,000

12,900

15,700

17,000

23.500

25,0

38,200

Vertical 4 - 1015 hours

$\begin{array}{rrr}26.0 & 9.300 & 11.7 \\ 26.0 & 9.300 & 11.7 \\ 26.0 & 9,700 & 11.6 \\ 26.0 & 14.600 & 11.5\end{array}$

$\begin{array}{cccc}\begin{array}{c}\text { ELEVATION, } \\ \text { FEET }\end{array} & \text { TEMPER- } & \begin{array}{c}\text { SPECIFIC } \\ \text { CONDUCT- }\end{array} & \begin{array}{c}\text { DISSOLVED } \\ \text { OXYGEN } \\ \text { (MILLI- }\end{array} \\ \begin{array}{c}\text { ABOVE (+) } \\ \text { OR BELOW(-) }\end{array} & \begin{array}{c}\text { ATURE } \\ \left({ }^{\circ} \mathrm{C}\right)\end{array} & \begin{array}{c}\text { ANCE } \\ \text { (MICRO- }\end{array} & \text { GRAMS PER } \\ \text { MEAN SEA } & & \text { MHOS AT } & \text { LITER) } \\ \text { LEVEL } & & \left.25^{\circ} \mathrm{C}\right) & \end{array}$

Cross Section F-F'

October 3, 1967b/--Continued

Vertical 4 - 1015 hours - Cont inued

$\begin{array}{llll}-7.7 & 26.0 & 15,600 & 10.6\end{array}$

$\begin{array}{llll}-9.7 & 26.0 & 16,800 & 10.5\end{array}$

$\begin{array}{rrrr}-11.7 & 26.0 & 23.500 & 8.2 \\ -13.7 & 26.5 & 39.200 & .7\end{array}$

Vertical $5-1035$ hours

$\begin{array}{llll}+1.5 & 26.0 & 9.400 & 10.9\end{array}$

$\begin{array}{llll}-1.3 & 26.0 & 9,400 & 10.9\end{array}$

$\begin{array}{llll}-3.3 & 25.5 & 11,200 & 10.9\end{array}$

$\begin{array}{llll}-5.3 & 25.5 & 12,800 & 10.8\end{array}$

$\begin{array}{llll}-7.3 & 25.0 & 14,600 & 10.8\end{array}$

$\begin{array}{llll}-9.3 & 25.5 & 16,600 & 9.8\end{array}$

$\begin{array}{llll}-11.3 & 25.0 & 23,500 & 7.2\end{array}$

$\begin{array}{rrrr}-13.3 & 25.5 & 37,300 & .6\end{array}$

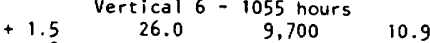

$\begin{array}{rrrr}+ & 26.0 & 9,700 & 10.9\end{array}$

$\begin{array}{llll}-4.8 & 25.5 & 10,700 & 10.9\end{array}$

$\begin{array}{llll}-6.8 & 25.5 & 12,400 & 10.9\end{array}$

$\begin{array}{llll}-8.8 & 25.5 & 12,400 & 10.9\end{array}$

$\begin{array}{llll}-10.8 & 25.0 & 23,500 & 7.8\end{array}$

$\begin{array}{llll}-12.8 & 25.0 & 28,500 & 4.4\end{array}$

+1.5 Vertical 7 - 1115 hours

$\begin{array}{rrrr}-26.5 & 8,200 & 9.7 \\ -2.8 & 26.5 & 8,300 & 10.0\end{array}$

$\begin{array}{rrrr}-4.8 & 27.0 & 9,000 & 9.7\end{array}$

$\begin{array}{rrrr}-6.8 & 27.0 & 11,600 & 10.2 \\ -6.8 & 26.0\end{array}$

$\begin{array}{llll}-8.8 & 26.0 & 12,600 & 10.4\end{array}$

$\begin{array}{llll}-10.8 & 26.0 & 23,500 & 7.2\end{array}$

$\begin{array}{llll}-12.8 & 26.0 & 26,500 & 4.7\end{array}$

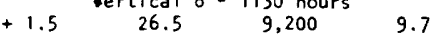

$\begin{array}{llll}-2.3 & 26.5 & 9,200 & 9.200\end{array}$

$\begin{array}{llll}-4.3 & 26.5 & 9,200 & 9.2\end{array}$

$\begin{array}{llll}-6.3 & 27.0 & 11.800 & 10.7\end{array}$

$\begin{array}{llll}-8.3 & 26.5 & 13,100 & 9.7\end{array}$

$\begin{array}{llll}-10.3 & 26.0 & 24,500 & 6.7\end{array}$

$\begin{array}{llll}-12.3 & 26.5 & 27.500 & 4.2\end{array}$

$\begin{array}{rrrr} & \text { Vertical } 9-1145 \text { hours } \\ +1.5 & 26.0 & 6,900 & 10.5\end{array}$

$\begin{array}{rrrr}.8 & 26.0 & 7,700 & 10.8\end{array}$

$\begin{array}{llll}-2.8 & 26.5 & 11,800 & 11.1\end{array}$

$\begin{array}{llll}-4.8 & 26.5 & 11,800 & 10.6\end{array}$

$\begin{array}{llll}-6.8 & 26.0 & 14,100 & 10.0\end{array}$

$\begin{array}{llll}-8.8 & 25.5 & 25,500 & 7.3\end{array}$

$\begin{array}{llll}-10.8 & 25.5 & 26,300 & 5.4\end{array}$

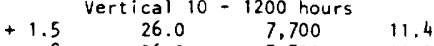

$\begin{array}{rrrr}+1.5 & 26.0 & 7,700 & 11.4 \\ -.8 & 26.0 & 7.700 & 11.0\end{array}$

$\begin{array}{rrrr}-2.8 & 26.0 & 9,000 & 9.6\end{array}$

$\begin{array}{llll}-4.8 & 26.0 & 11,900 & 9.6\end{array}$

$\begin{array}{llll}-6.8 & 26.0 & 12,100 & 9.6\end{array}$

$\begin{array}{cccc}+1.5 & 26.0 & 7.400 & 11.4\end{array}$

$\begin{array}{llll}-3.3 & 25.5 & 10,700 & 10.5\end{array}$

$\begin{array}{rrrr}-8.3 & 25.0 & 13,100 & 8.8 \\ -13.3 & 25.0 & 21.600 & 6.5\end{array}$

See fnotnotes at end of table. 
TABLE 12.-Field determinations of water-quality data for Corpus Christi Bay, October 2-3, 1967-Continued

$\begin{array}{cccc}\text { ELEVATION, } & & \text { SPECIFIC } & \text { DISSOLVED } \\ \text { FEET } & \text { TEMPER- } & \text { CONDUCT- } & \text { OXYGEN } \\ \text { ABOVE }(+) & \text { ATURE } & \text { ANCE } & \text { (MILLI- } \\ \text { OR BELOW(-) } & \left({ }^{\circ} \mathrm{C}\right) & \text { (MICRO- } & \text { GRAMS PER } \\ \text { MEAN SEA } & & \text { MHOS AT } & \text { LITER) } \\ \text { LEVEL } & & \left.25^{\circ} \mathrm{C}\right) & \end{array}$

Cross Section F-F'

October 3, 1967b/--Cont inued

$\begin{array}{lccc}\text { Vertical } & 11- & 1230 & \text { hours--Continued } \\ -18.3 & 25.0 & 26,500 & 3.8 \\ -23.3 & 25.5 & 30,500 & 3.5 \\ -28.3 & 26.0 & 34,400 & 2.6 \\ -33.3 & 26.0 & 39,200 & 1.9 \\ -38.3 & 26.0 & 41,100 & 1.8\end{array}$

Cross Section G-G' - October 3, 1967b/

$\begin{array}{cccc} & \text { Vertical 1 } & \text { 0945 hours } \\ +0.8 & 26.1 & 23,600 & 7.8 \\ -1.4 & 25.6 & 23,600 & 7.9 \\ + & \text { Vertical 2 } & 0930 \text { hours } & \\ +0.8 & 25.6 & 21,500 & 7.8 \\ -1.0 & 25.6 & 22,500 & 7.4 \\ +0.7 & \text { vertical 3 } & 1035 \text { hours } & \\ -8.3 & 26.1 & 19,900 & 7.1 \\ -15.0 & 25.6 & 20,200 & 7.4 \\ +0.7 & 26.1 & 20,400 & 7.6 \\ -4.3 & 26.7 & 16,300 & 7.2 \\ & 26.7 & 16,300 & 7.3\end{array}$

Cross Section H-H' - October 3, 1967b/

\begin{tabular}{|c|c|c|}
\hline \multirow{7}{*}{$\begin{array}{l}+0.7 \\
-3.3 \\
-5.3 \\
-6.3 \\
-7.3 \\
-9.3\end{array}$} & \multirow{7}{*}{$\begin{array}{c}\text { Vertical } 1- \\
27.8 \\
26.7 \\
26.7 \\
26.7 \\
26.7 \\
26.7\end{array}$} & \multirow{7}{*}{$\begin{array}{c}1320 \text { hours } \\
c / 16,700 \\
c / 17,000 \\
c / 17,200 \\
c / 17,200 \\
c / 17,500 \\
c / 18,000\end{array}$} \\
\hline & & \\
\hline & & \\
\hline & & \\
\hline & & \\
\hline & & \\
\hline & & \\
\hline & Vertical 2 & - 1350 hours \\
\hline 0.7 & 26.7 & 18,100 \\
\hline-3.3 & 26.7 & 18,300 \\
\hline-5.3 & 26.7 & 18,300 \\
\hline-6.3 & 26.7 & 18,300 \\
\hline 8. & 26.1 & 19,900 \\
\hline & 25.6 & 21,000 \\
\hline & & 21,000 \\
\hline & Vertical 4 & - 1415 hours \\
\hline+0.7 & 27.2 & 15,600 \\
\hline-3.3 & 27.2 & 15,600 \\
\hline-5.3 & 27.2 & 15,600 \\
\hline$=0$ & 25.6 & 15,600 \\
\hline & ical 7 & - 1530 hours \\
\hline 1.5 & 27.0 & 18,100 \\
\hline & 27.0 & 18,100 \\
\hline 2.3 & 27.0 & 18,100 \\
\hline-4.3 & 26.5 & 18,100 \\
\hline 6.3 & 25.0 & 24,200 \\
\hline & tical 9 & - 1545 hours \\
\hline 1.6 & 26.5 & 18,100 \\
\hline-.2 & 26.5 & 18,100 \\
\hline 2.2 & 26.5 & 18,100 \\
\hline 4.2 & 26.5 & 18,300 \\
\hline & erticai ${ }^{25}$ & $\begin{array}{r}23,600 \\
-\quad 1615 \text { hou }\end{array}$ \\
\hline & 27.0 & \\
\hline
\end{tabular}

$\begin{array}{cccc}\text { ELEVATION, } & & \text { SPECIFIC } & \text { DISSOLVED } \\ \text { FEET } & \text { TEMPER- } & \text { CONDUCT- } & \text { OXYGEN } \\ \text { ABOVE }(+) & \text { ATURE } & \text { ANCE } & \text { (MILLI- } \\ \text { OR BELOW }(-) & \left({ }^{\circ} \mathrm{C}\right) & \text { (MICRO- } & \text { GRAMS PER } \\ \text { MEAN SEA } & & \text { MHOS AT } & \text { LITER) } \\ \text { LEVEL } & & & \end{array}$

Cross Section $\mathrm{H}-\mathrm{H}^{\prime}$ October 3, 1967b/--Cont inued

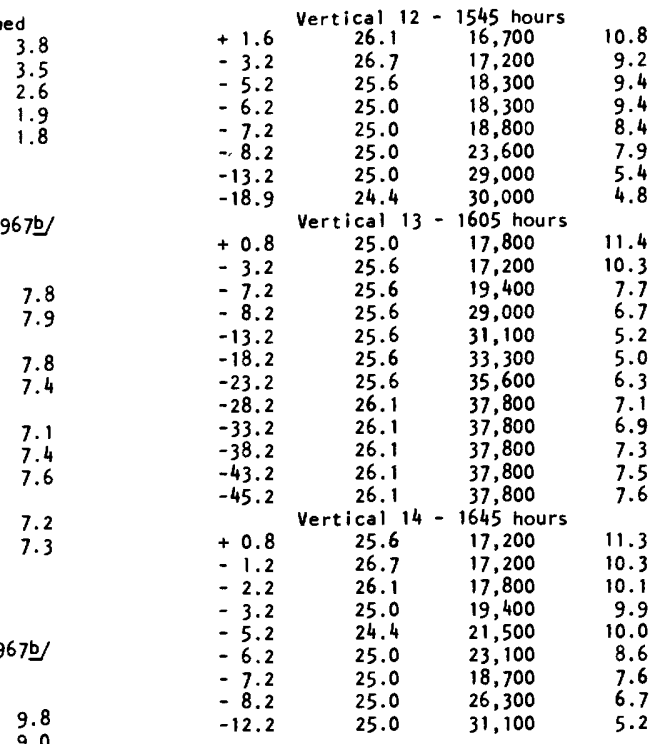

Cross Section 1-1'- October 3, 1967b/

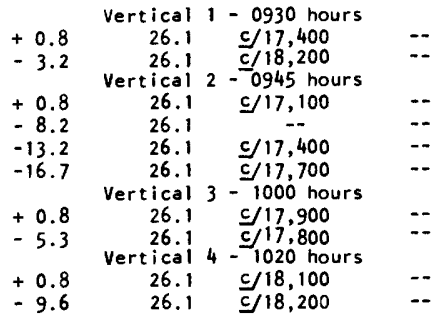

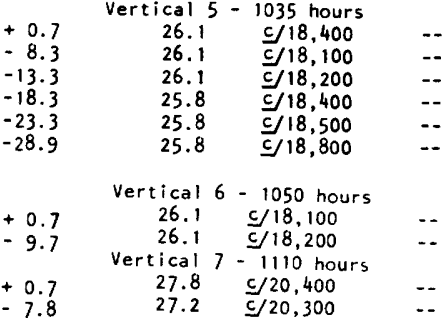

a) Stage of bay fluctuated from +1.9 feet at 1400 hours to +2.0 feet at 1700 hours to +1.9 feet at 1900 hours.

b) Stage of bay fluctuated from +1.8 feet at 0900 hours to +1.6 feet at 1500 hours to +1.8 feet at 1700 hours.

c) Determined in laboratory. 
SOUTH TEXAS AND NORTHEASTERN MEXICO, SEPT.-OCT.

ळ

¿.

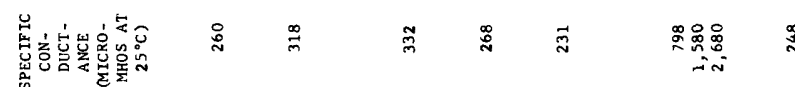

క్ర

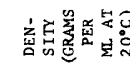

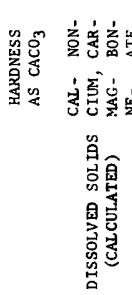

安究

它㽰色

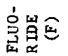

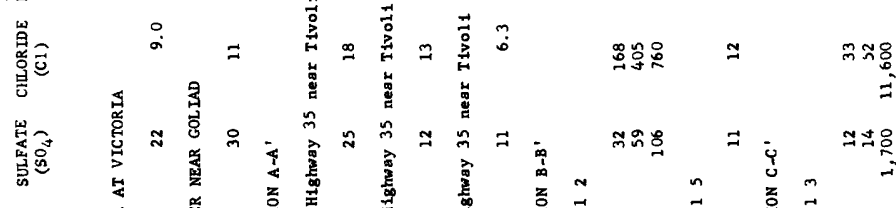

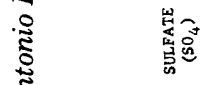

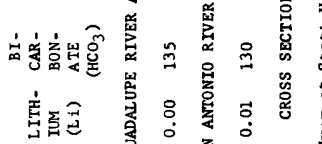

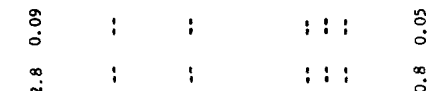

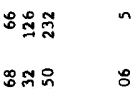

$\simeq=$

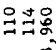

密

కี

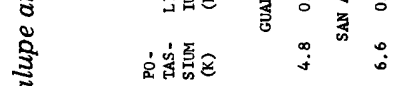

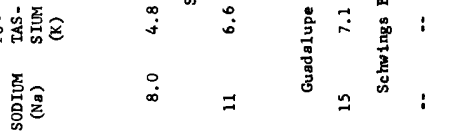

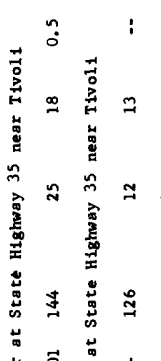

$: i: \quad m$

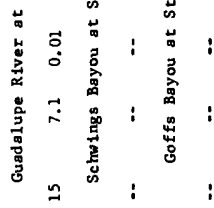

䇏鲄

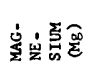

ริำํำำ

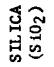

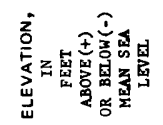

ร

ฮ ฮ

?an

i: : $:$

: : :

: : :

i: $\quad \stackrel{\infty}{i}$

: : : 文

$\stackrel{\infty}{\sigma}=\approx \quad \approx$

윯ำ

$i:$ ?

$: i_{\infty}^{\infty}$

1: : 昌

: :

$\because \because g$

$:: \stackrel{\square}{\pi}$

苛

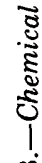

象

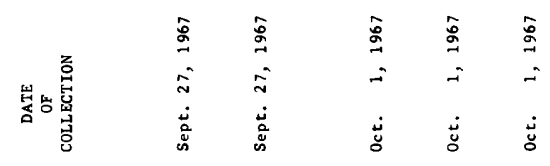

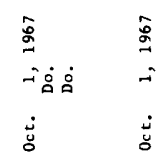

유ำ 


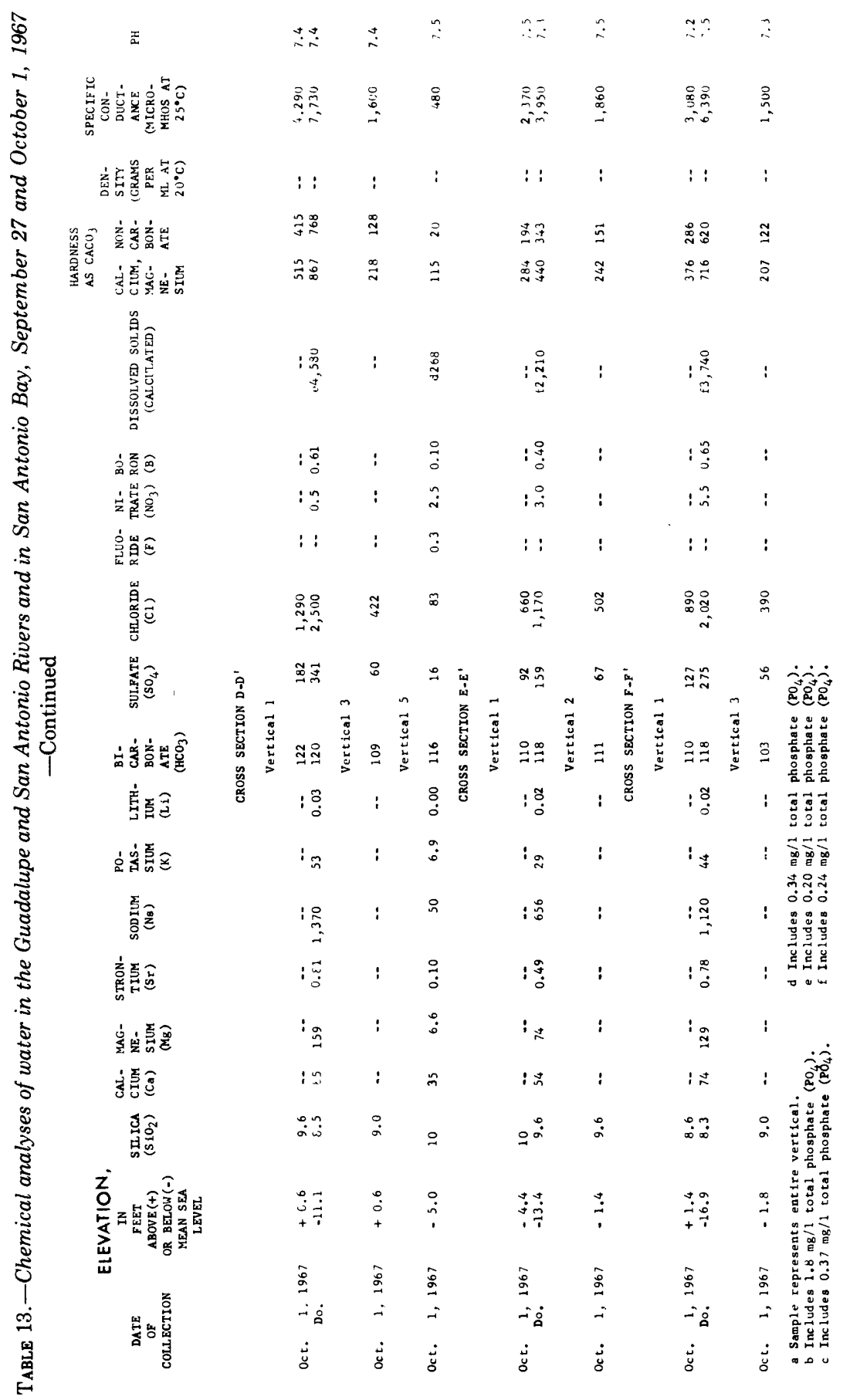




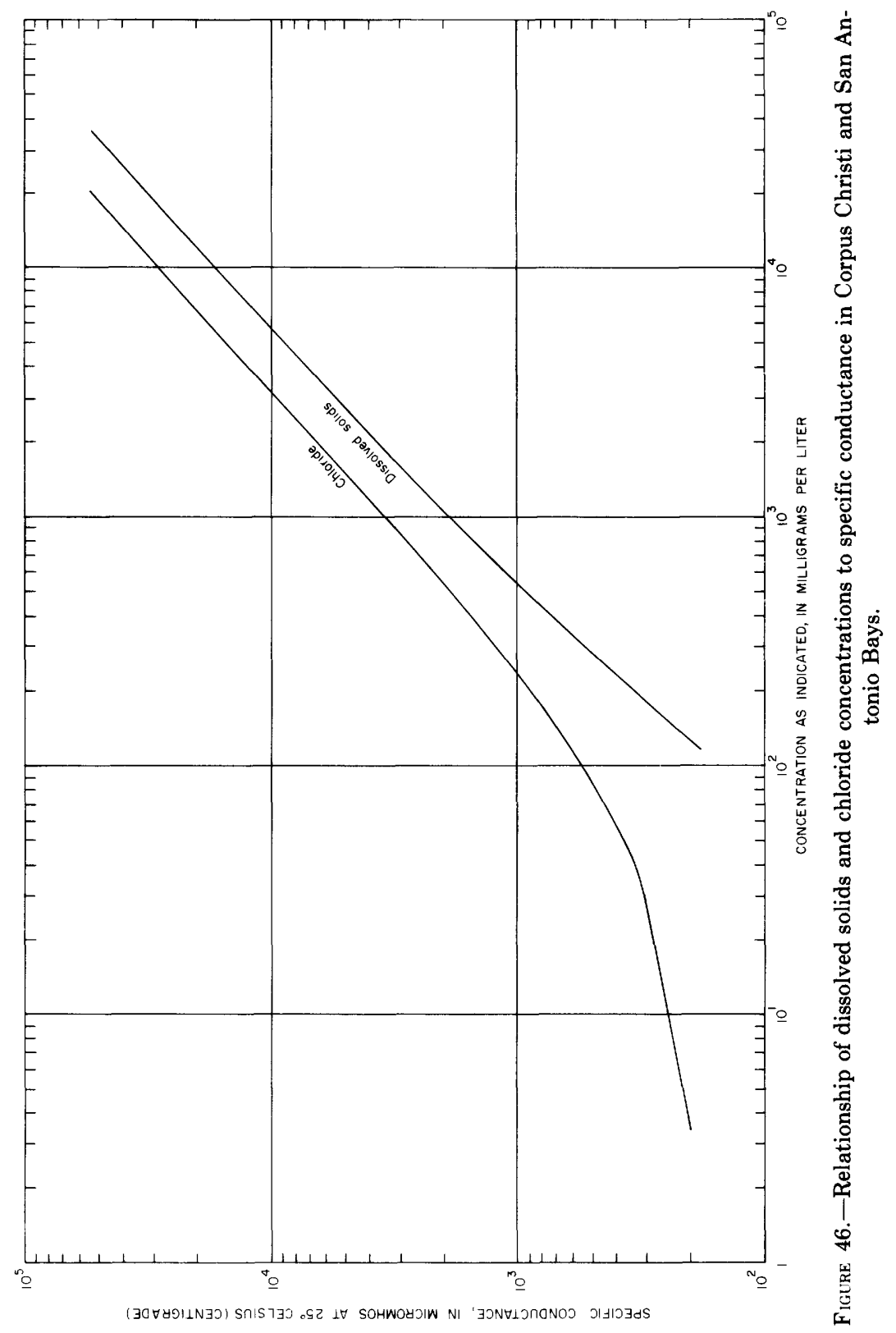


TABLE 14.-Field determinations of water-quality data for San Antonio Bay, October 1, 1967

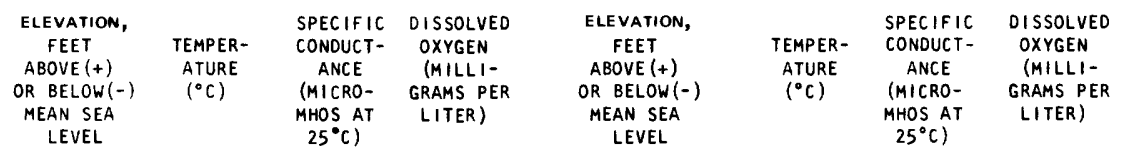

Cross Section B-B' - October 1, 1967a/

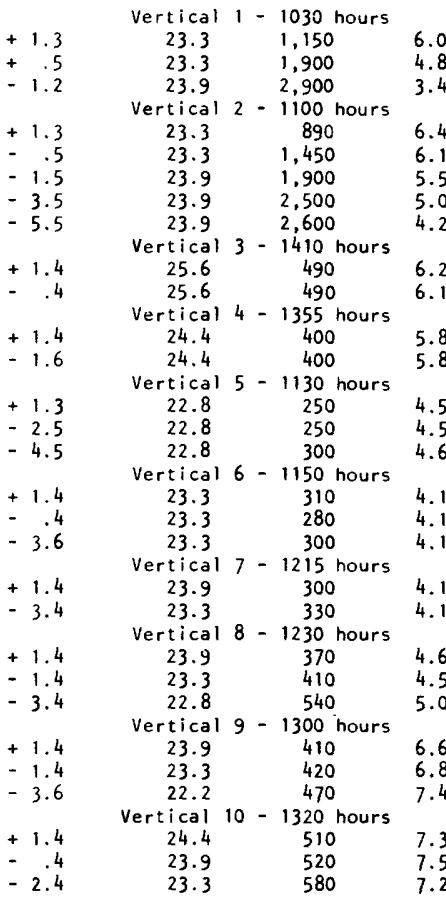

Cross Section C-C' - October 1, 1967a/

$\begin{array}{cccc} & \text { Vertical } & -1140 \text { hours } \\ +1.4 & 24.0 & 2,500 & 5.1 \\ -5.9 & 23.5 & 2,500 & 5.1 \\ -7.9 & 23.5 & 2,500 & 5.1 \\ -9.9 & 23.5 & 2,500 & 5.1 \\ -11.9 & 23.5 & 2,500 & 5.1 \\ & \text { Vertical } 2-1150 \text { hours } \\ +1.4 & 24.5 & 470 & 4.9 \\ -3.4 & 24.5 & 470 & 4.8 \\ +1.4 & \text { Vertical } 3-1200 \text { hours } \\ -3.4 & 24.0 & 370 & 4.8 \\ -5.4 & 24.0 & 370 & 4.8 \\ -7.4 & 24.0 & 390 & 4.8 \\ -9.4 & 24.0 & 400 & 4.8 \\ -11.4 & 24.0 & 4,300 & 4.7 \\ & 25.5 & 31,500 & 1.9\end{array}$

See footnote at end of table.
Cross Section $\mathrm{C}-\mathrm{C}^{\prime}$

October 1, 1967a/--Continued

+1.4
-5.9
+1.4
-4.4
+1.4
-2.4

$\begin{array}{rcc}\text { Vertical } & 4-1220 \text { hours } & \\ 24.5 & 330 & 4.9 \\ 25.5 & 330 & 4.7 \\ \text { Vertical } & 5-1230 \text { hours } & \\ 24.5 & 550 & 4.7 \\ 24.5 & 610 & 4.4 \\ \text { Vertical } & 6-1240 \text { hours } & \\ 24.5 & 850 & 4.9 \\ 25.0 & 950 & 4.8\end{array}$

Cross Section D-D' - October 1, 1967a/

$+0.6$

-3.4
-8.4

$-11.1$

$+.6$

$-1.4$

$-2.9$

$-4.4$

$+.6$

$-1.4$

$-3.4$

$-4.4$

$+0.7$

$-3.3$

- 6.3

$+.7$

-1.3
-5.0

Vertical 1 - 1920 hours

$25.0 \quad 4,300$

6.8

$23.9 \quad 4,600$

$25.0 \quad 6,000$

6.8

Vertical 2 - 1545 hours

$\begin{array}{lll}24.4 & 1,800 & 7.5\end{array}$

$24.4 \quad 1,800$

$24.4 \quad 2,200$

$23.9 \quad 4,300$

Vertical 3 - 1605 hours

$\begin{array}{lll}24.4 & 1,600 & 7.7\end{array}$

$\begin{array}{lll}24.4 & 1,600 & 7.7\end{array}$

$24.4 \quad 1,700 \quad 7.7$

$24.4 \quad 2,500 \quad 5.1$

Vertical 4 - 1630 hours

$\begin{array}{ccc}24.4 & 670 & 6.7\end{array}$

$\begin{array}{lll}23.9 & 700 & 6.9\end{array}$

$\begin{array}{cc}23.3 & 880 \\ \text { Vertical } 5 & -1650 \text { hours }\end{array}$

$\begin{array}{lll}24.4 & 490 & 6.7\end{array}$

$\begin{array}{rrrr}-5.4 & 24.4 & 490 & 6.7 \\ -5.0 & 22.8 & 520 & 7.0\end{array}$

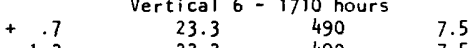

$\begin{array}{rrrr}+1.7 & 23.3 & 490 & 7.5 \\ -4.3 & 23.3 & 490 & 7.5\end{array}$

$-4.3$

$+0.6$

+
-3.4

Vertical 7 - 1730 hours

$\begin{array}{lll}23.3 & 710 & 7.5\end{array}$

$\begin{array}{ccc}22.8 & 920 & 7.4 \\ \text { Vertical } 8-1755 \text { hours } & \end{array}$

$\begin{array}{lll}24.4 & 870 & 7.5\end{array}$

$\begin{array}{rrrr}+.6 & 24.4 & 7.5 \\ -3.4 & 24.4 & 870 & 7.5\end{array}$

$\begin{array}{rrrr}-5.1 & 24.4 & 1,070 & 7.0\end{array}$

$\begin{array}{rrrr} & \text { Vertical } 9-1815 \text { hours } & \\ +.6 & 25.6 & 1,170 & 8.0 \\ -1.8 & 25.6 & 1,120 & 7.9\end{array}$

Cross Section E-E' - October 1, 1967a/

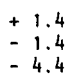

Vertical 1 - 1800 hours

$\begin{array}{lll}25.5 & 2,300 & 4.2 \\ 25.0 & 2,300 & 4.4 \\ 24.5 & 2,300 & 4.2\end{array}$


TABLE 14.-Field determinations of water-quality data for San Antonio Bay, October 1, 1967-Continued

$\begin{array}{llll}\text { ELEVATION, } & & \text { SPECIFIC } & \text { DISSOLVED } \\ \text { FEET } & \text { TEMPER- } & \text { CONDUCT- } & \text { OXYGEN } \\ \text { ABOVE }(+) & \text { ATURE } & \text { ANCE } & \text { (MILLI- } \\ \text { OR BELOW }(-) & \left({ }^{\circ} \mathrm{C}\right) & \text { (MICRO- } & \text { GRAMS PER } \\ \text { MEAN SEA } & & \text { MHOS AT } & \text { LITER) } \\ \text { LEVEL } & & \left.25^{\circ} \mathrm{C}\right) & \end{array}$

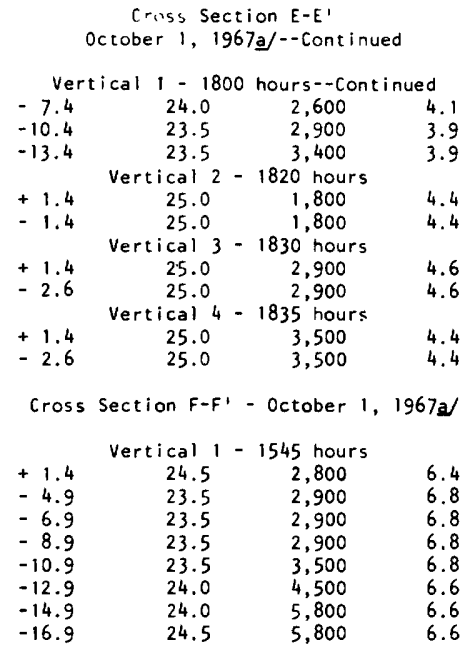

$\begin{array}{lccc}\text { ELEVATION, } & & \text { SPECIFIC } & \text { DISSOLVED } \\ \text { FEET } & \text { TEMPER- } & \text { CONDUCT- } & \text { OXYGEN } \\ \text { ABOVE (+) } & \text { ATURE } & \text { ANCE } & \text { (MILLI- } \\ \text { OR BELOW(-) } & \left({ }^{\circ} \mathrm{C}\right) & \text { (MICRO- } & \text { GRAMS PER } \\ \text { MEAN SEA } & & \text { MHOS AT } & \text { LITER) } \\ \text { LEVEL } & & \left.25^{\circ} \mathrm{C}\right) & \end{array}$

Cross Section $F-F^{\prime}$

october 1, 1967a/--Continued

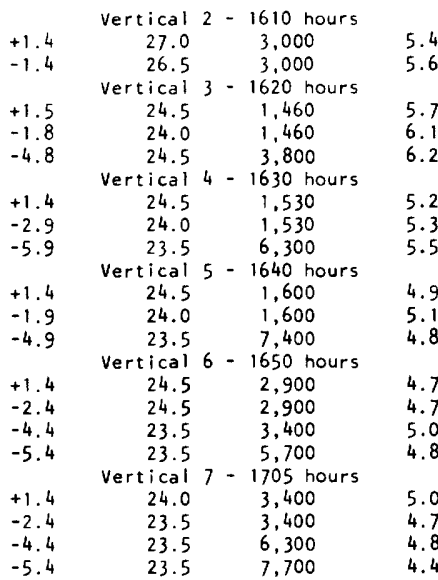

a) Stage of bay fluctuated from +1.5 feet at 1000 hours to +1.7 feet at 1700 hours to +1.6 feet at 2000 hours.

Under the influence of the tremendous influx of fresh water, the salinity of water in the bays was greatly reduced. Because the flood waters moved directly thorugh each bay, dilution proceeded rapidly along the line of flow. The side bays and barge channels of San Antonio Bay, though diluted, were bypassed by the main floodflow. Even a depression in the bay floor was left filled with saline water, while a few feet above, the water was fresh. In Corpus Christi Bay, even though the entire bay was freshened, the flow of dilute water was directly through the bay. After flooding ceased and a windstorm moved over the bay, lateral and in-depth dilution increased significantly.

The barge channels in both bays and most of the Corpus Christi ship channel seemed to have little influence on the movements of flood waters. Only that part of the ship channel near Aransas Pass seemed to have an influence on flow of water.

Differences in configuration, depth, and volumes of inflow relative to the size of each bay were largely responsible for a higher concentration of dissolved solids in Corpus Christi Bay. 


\section{NUECES-CORPUS CHRISTI BAY}

Nueces-Corpus Christi Bay is on the Texas gulf coast in Nueces County (fig. 47). Corpus Christi Bay is roughly an oval about 15 miles by 10 miles, with a uniform depth of 10 to 13 feet. Nueces Bay, which is about 3 miles wide and generally less than 3 feet deep, extends inland about 7 miles from its junction with Corpus Christi Bay. Deepwater access to the Gulf of Mexico is maintained by a 45-foot-deep channel between Corpus Christi and Port Aransas. The Nueces River, with a total drainage area of approximately 17,000 square miles, discharges into Nueces Bay at Corpus Christi.

Data were collected twice during the investigation on Corpus Christi Bay, once when flow in the Nueces River near Calallen, immediately upstream from Nueces Bay, was about 100,000 cfs and once when the flow was about 26,000 cfs.

During the period September $28-30,1967$, water in the Nueces River near Calallen contained about $122 \mathrm{mg} / 1$ (milligrams per liter) dissolved solids, most of which were calcium and bicarbonate ions. By the time the water had flowed through Nueces Bay, it contained $184 \mathrm{mg} / \mathrm{l} \mathrm{dis-}$ solved solids and was a calcium sodium bicarbonate type.

Daily inflow to Corpus Christi Bay exceeded 60,000 cfs from September 23 through September 28. The total inflow was about $1 \frac{112}{2}$ times the volume of water normally in the bay, but because of its shape and depth, the bay was not entirely flushed of saline water. The stream of dilute water moved generally from the confluence of Nueces and Corpus Christi Bays about one-third of the way to the Naval Air station, then along the ship channel and out of the bay through Aransas Pass.

As the water moved through Corpus Christi Bay from the bridge at U. S. Highway 181 to the position of traverse line $D-D^{\prime}$ (fig. 47), it changed from a calcium sodium bicarbonate type with $184 \mathrm{mg} / \mathrm{l}$ dissolved solids to a sodium chloride type with $333 \mathrm{mg} / \mathrm{l}$ dissolved solids, and magnesium and sulfate became more abundant than calcium and bicarbonate. The water left the bay with a concentration of about 7,000 $\mathrm{mg} / 1$ dissolved solids. The changes in concentrations as the water moved through the bay are shown on plate 3 .

The concentrations of strontium and lithium (table 9), determined at a few points in the bay, were generally very low, but increased at about the same rate as chloride and sulfate. The phosphate and nitrate concentrations, probably affected by biologic activity, varied irregularly from point to point. Dissolved-oxygen concentrations in much of the water near the surface of the bay was about $8 \mathrm{mg} / \mathrm{l}$. This was 2 to $3 \mathrm{mg} / \mathrm{l}$ greater than at depth. The difference between surface and bottom dissolved-oxygen concentrations reached $6 \mathrm{mg} / \mathrm{l}$ in only one place.

The second set of data were collected in Corpus Christi Bay during the period October 2-3, 1967 (tables 11, 12; pl. 3). At this time, flow in 


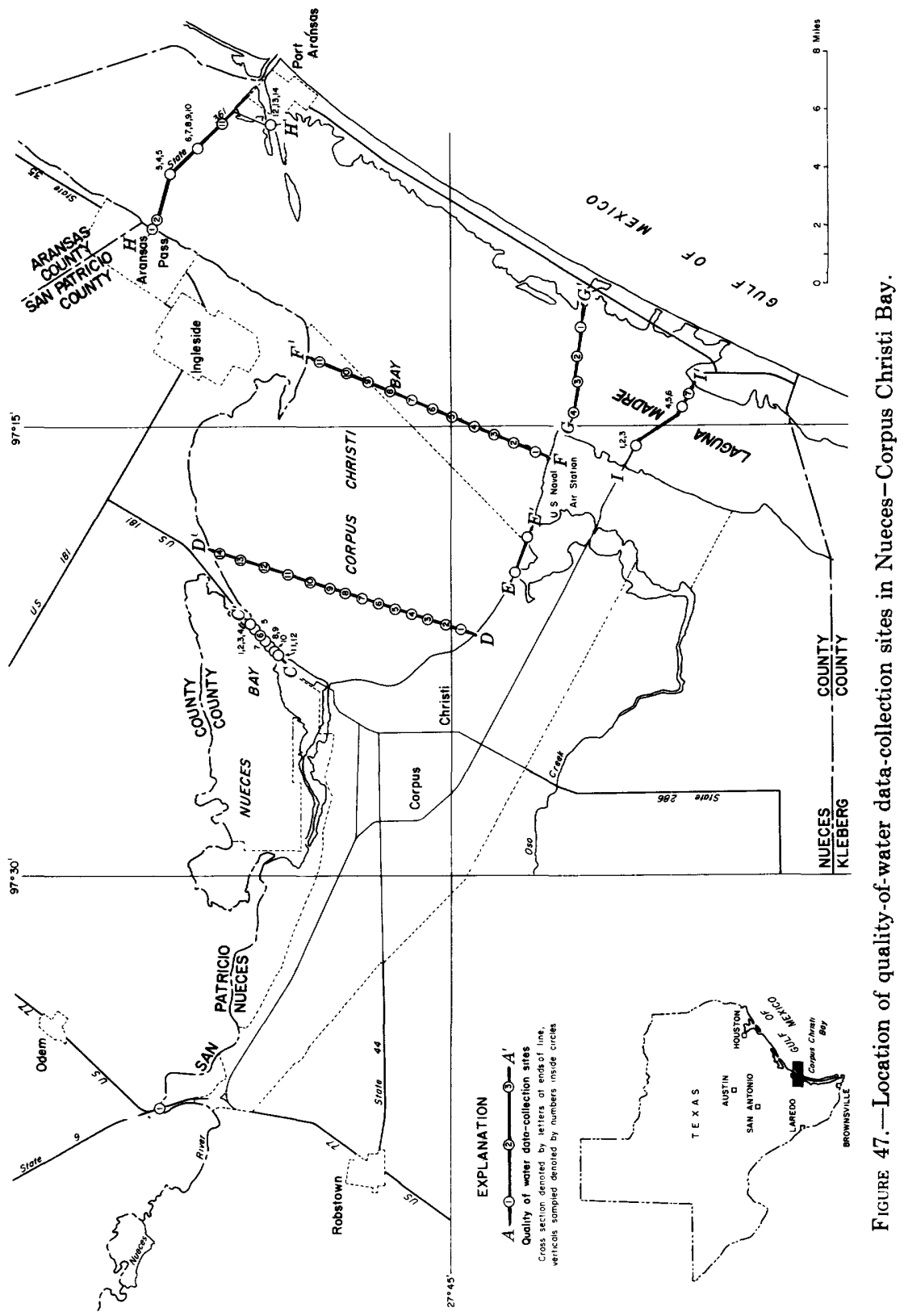


the Nueces River was approximately $26,000 \mathrm{cfs}$ or about one-fourth of the flow during the previous data-collection period.

Water in the Mathis-Calallen reach of the Nueces River contained about $145 \mathrm{mg} / \mathrm{l}$ dissolved solids. Along the line of contact between Corpus Christi Bay and Nueces Bay, the concentration of dissolved solids ranged from 300 to $1,200 \mathrm{mg} / \mathrm{l}$. At Aransas Pass, the water left the bay as a sodium chloride type with a dissolved-solids concentration of about $10,000 \mathrm{mg} / \mathrm{l}$. Throughout most of the bay, the second most predominant pair of ions were magnesium and sulfate.

As in the first analyses, concentrations of lithium and strontium increased at about the same rate as chloride and sulfate, and nitrate concentrations were irregular.

The dissolved-oxygen content of much of the water near the surface of the bay was about $10 \mathrm{mg} / \mathrm{l}$ (pl. 3; table 12). At depth the dissolvedoxygen content varied markedly and was as much as 8 to $10 \mathrm{mg} / \mathrm{l}$ lower than at the surface. In a few places, dissolved oxygen was as low as 0.5 $\mathrm{mg} / \mathrm{l}$. This was a complete change from the conditions noted a few days earlier.

Although the stage of Corpus Christi Bay was almost the same during the two data-collection periods (fig. 45), the conditions causing the stage were different. During the September 28-30 period, the bay was receiving a very large volume of freshwater inflow. During the October 2-3 period, the volume of inflow was much smaller but the stage was maintained by winds.

The September chemical-quality data show that a large volume of dilute water was moving directly through the bay, with little mixing away from the line of flow or at depth. The October data show the combined effects of wind and reduced inflow. Mixing was more uniform throughout the bay and extended to greater depths. Both sets of data indicate little, if any, mixing in the deeper parts of the channels.

Suspended-sediment samples were taken from the Nueces River near Mathis and near Calallen, and from Corpus Christi Bay at its confluence with Nueces Bay (table 8). Analysis of the samples indicate that after the floodwater entered Nueces Bay, the velocity of flow decreased enough to allow most of the suspended sediment to settle out, leaving Corpus Christi Bay relatively free of flood-derived sediment.

The velocity of flow in the middle of Corpus Christi Bay ranged from approximately $1 \mathrm{fps}$ (foot per second) at the surface to almost zero at the bottom. At some points, the direction of flow at depth was opposite from the direction at the surface. This reversal can be attributed to either tidal currents or density currents. In Nueces Bay, the velocity of flow was as much as $3 \mathrm{fps}$. Velocities in the ship channel were measured as follows: In the middle of the bay, zero; near Ingleside, from 1 to $2 \mathrm{fps}$; and near Aransas Pass, 1.7 fps. 


\section{GUADALUPE-SAN ANTONIO BAY}

Guadalupe-San Antonio Bay is on the Texas gulf coast in Calhoun County (fig. 48). The depth of water in San Antonio Bay averages about 5 feet; deep water access to the gulf is through Victoria Channel and Intracoastal Waterway via Port O'Connor. The Guadalupe River, with a total drainage area (including the tributary San Antonio River) of approximately 10,000 square miles, discharges into Guadalupe Bay.
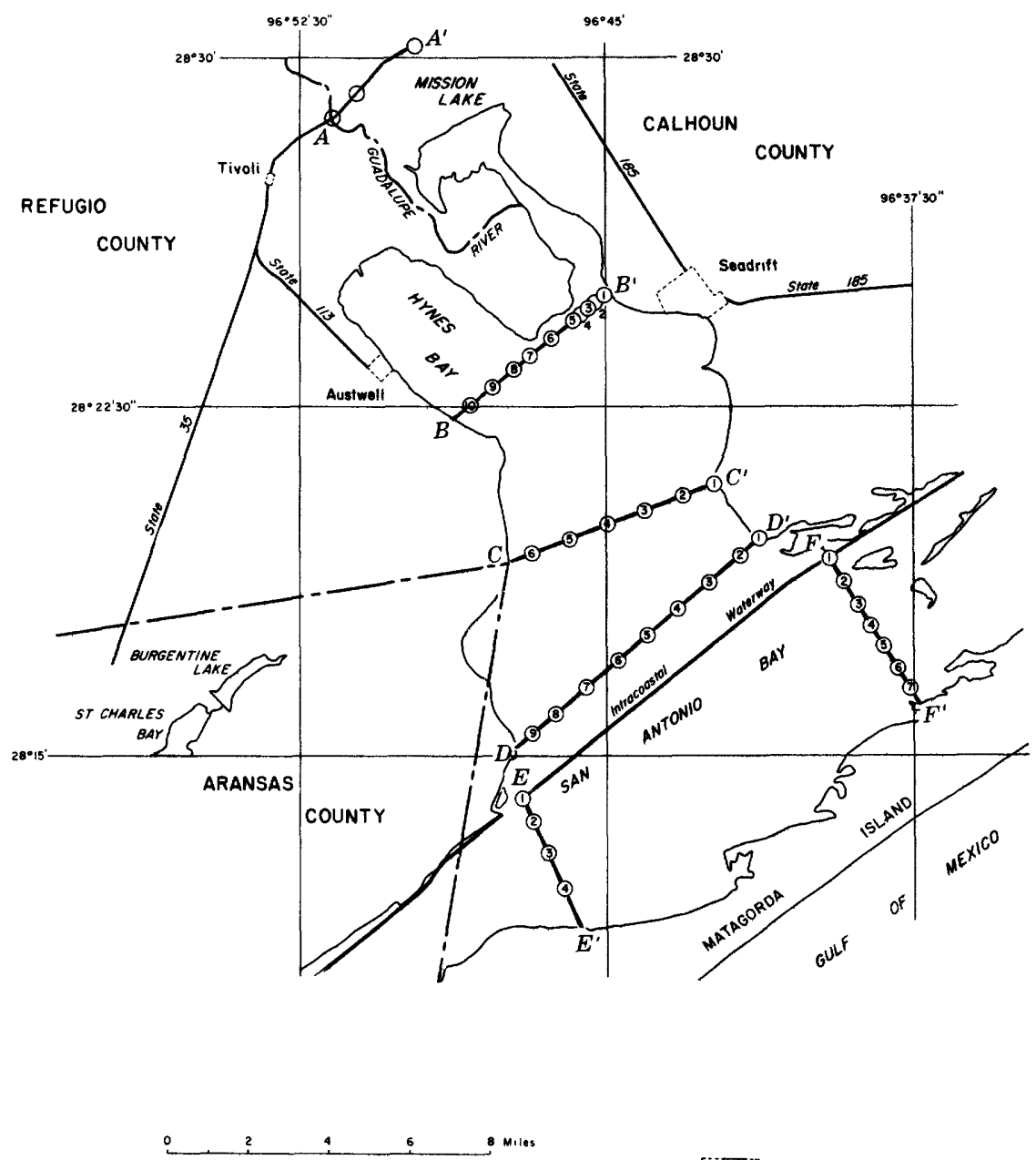

EXPLANATION

$E-(1)-(2)-E^{\prime}$

Quelity of water doto-collection sites

Crose section denoted by letters of ends of line, verticols sampled denoted by numbers msice curcles

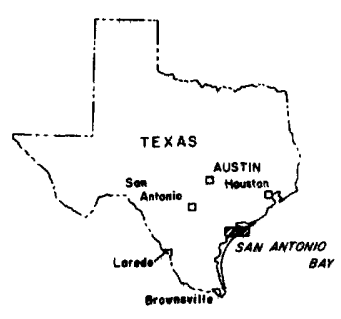

FiguRe 48.-Location of quality-of-water data-collection sites in San Antonio Bay. 
Data were collected only once, on October 1,1967 , during the investigation of San Antonio Bay. At that time, the flow of the Guadalupe River near Tivoli (fig. 63) was about $23,000 \mathrm{cfs}$, and the water was a calcium bicarbonate type with a dissolved-solids concentration of 208 $\mathrm{mg} / \mathrm{l}$. Floodwaters in other stream channels near Tivoli contained as little as $150 \mathrm{mg} / \mathrm{l}$ dissolved solids.

The field data (table 14) indicate that the diluted water was moving down the center of San Antonio Bay toward Matagorda Island, then generally northeast into the Gulf of Mexico at Pass Cavallo. The water did not flow along either Victoria Channel or the Intracoastal Waterway because the stage of the bay was high enough to inundate the islands that normally restrict the movement of water from San Antonio to Matagorda Bay.

The water at the head of the bay was a calcium bicarbonate type with a dissolved-solids concentration of $152 \mathrm{mg} / \mathrm{l}$ (table 13). Seaward, the concentration increased. The changes in dissolved-solids and chloride concentrations are shown on plate 3 .

The freshwater inflow to San Antonio Bay exceeded 40,000 cfs from September 21 through September 26, with the total inflow more than three times the volume of water normally in the bay. Most of the saline water was flushed out of the bay.

Dilution of the saline water was most rapid along the line of flow. Water in Victoria Channel and in bay-floor depressions was least affected by dilution (pl. 3). Concentrations of strontium and lithium increased in proportion to sodium and chloride, and phosphate and nitrate concentrations were irregular; the concentration of dissolved oxygen varied little with depth, but increased along the line of flow from 4 $\mathrm{mg} / 1$ to more than $7 \mathrm{mg} / \mathrm{l}$, then decreased to about $6 \mathrm{mg} / 1$ (pl. 3 ; table 14).

\section{EFFECTS OF THE HURRICANE ON WATER LEVELS IN WELLS}

By Texas Water Development Board

Soon after the floodwaters of Hurricane Beulah began to subside, personnel of the Texas Water Development Board set out to document the effects, if any, on the ground-water reservoirs.

Seventy-three representative water wells were selected for measurements of depth to water; these were wells for which preflood data were available, and most of them were less than 100 feet deep. All the wells, regardless of depth, might be expected to show rises in water levels, but the rise in the deep wells and in some of the shallow wells would probably be caused by cutbacks in pumping, rather than by recharge.

Water levels were measured in 59 wells in mid-October, and all 73 wells were measured in mid-November. The October, November, and 

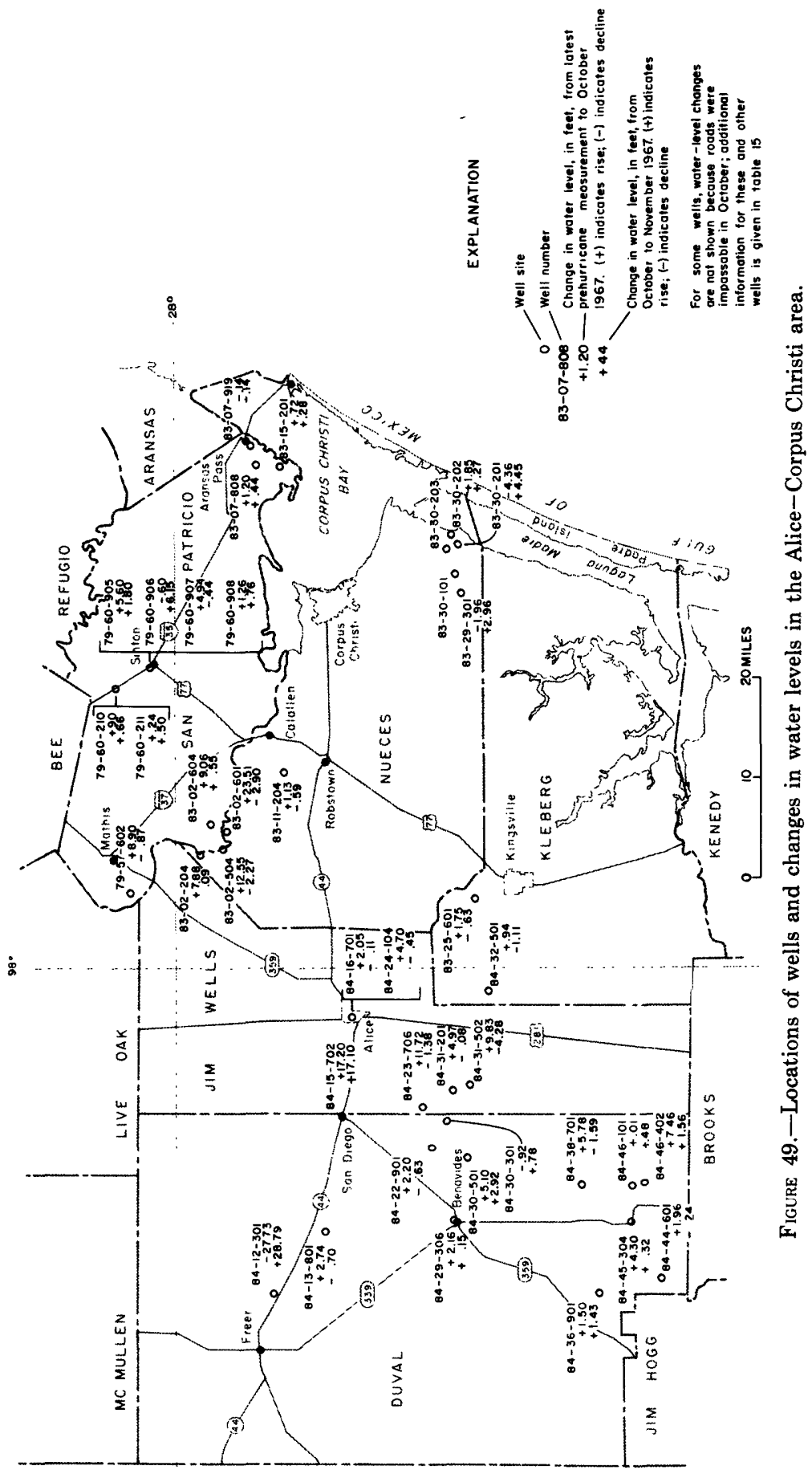
preflood measurements of depth to water are given in tables 15 and 16 . Well locations are shown in figures 49 and 50.

In the lower Rio Grande Valley (fig. 50), water-level measurements in the past years have usually been made in late summer, when irrigation pumpage is diminished. In the Alice-Corpus Christi area (fig. 49), where growing seasons are different, pumpage is usually minimum during the winter months, and the annual water-level measurements are generally obtained then.

Some of the data in tables 15 and 16 have been simplified in the following tabulation by averaging the water-level changes for wells that have similar dates of measurements. These are the wells that were accessible in October and for which earlier measurement dates are close together-February, 1967 for the Alice-Corpus Christi area and July, 1967 for the lower Rio Grande Valley. These averages and tables 15 and 16 show that in each region the water levels generally rose several feet prior to the October measurement and then generally declined slightly from the October to November measurements.

Average rise or decline of water level, in feet

[Plus (+) indicates rise; minus $(-)$ indicates decline]

\begin{tabular}{|c|c|c|}
\hline \multicolumn{3}{|c|}{ Alice-Corpus Christi area } \\
\hline $\begin{array}{l}18 \text { shallow wells }(\leq 100 \mathrm{ft} \text { deep) } \ldots \\
2 \text { deep wells }(>100 \mathrm{ft} \text { deep) } \ldots \ldots\end{array}$ & $\begin{array}{c}\text { Feb.-Oct., } 1967 \\
+4.39 \\
+3.33\end{array}$ & $\begin{array}{l}\text { Oct. }- \text { Nov., } 1967 \\
-0.16 \\
-.35\end{array}$ \\
\hline \multicolumn{3}{|c|}{ Lower Rio Grande Valley } \\
\hline $\begin{array}{l}8 \text { shallow wells } \ldots \ldots \ldots \ldots \ldots \ldots \ldots \\
11 \text { deep wells } \ldots \ldots \ldots \ldots \ldots \ldots \ldots\end{array}$ & $\begin{array}{c}\text { July-Oct., } 1967 \\
+12.40 \\
+7.80\end{array}$ & $\begin{array}{l}\text { Oct. }- \text { Nov., } 1967 \\
\quad-0.99 \\
-1.63\end{array}$ \\
\hline
\end{tabular}

Because water levels normally change in response to seasonal pumping, not all the rises in water levels can be attributed to rains from Hurricane Beulah. However, the significance of these rises, particularly in the shallow wells, may be determined by comparing them with water-level changes in previous years. Figures 51 and 52 are hydrographs of water-level fluctuations in selected wells for which earlier records are available.

In the Alice-Corpus Christi area, comparison of water-level fluctuations in well 83-02-504 (40 ft deep) and well 79-57-602 (205 ft deep) with previous trends shows major rises of water levels during the hurricane period. In contrast, well $84-16-701$ (844 ft deep) had only a slight rise in water level during the same period (fig. 51). Two other deep wells, 83-25-601 and 84-15-702, exhibit seasonal fluctuations that are large enough to mask any effects of the hurricane.

The hydrographs of wells 87-31-911 (91 ft deep) and 87-54-514 (115 ft deep) in the lower Rio Grande Valley (fig. 52) show marked rises in water levels during the September 1967 period. Slight rises above the 


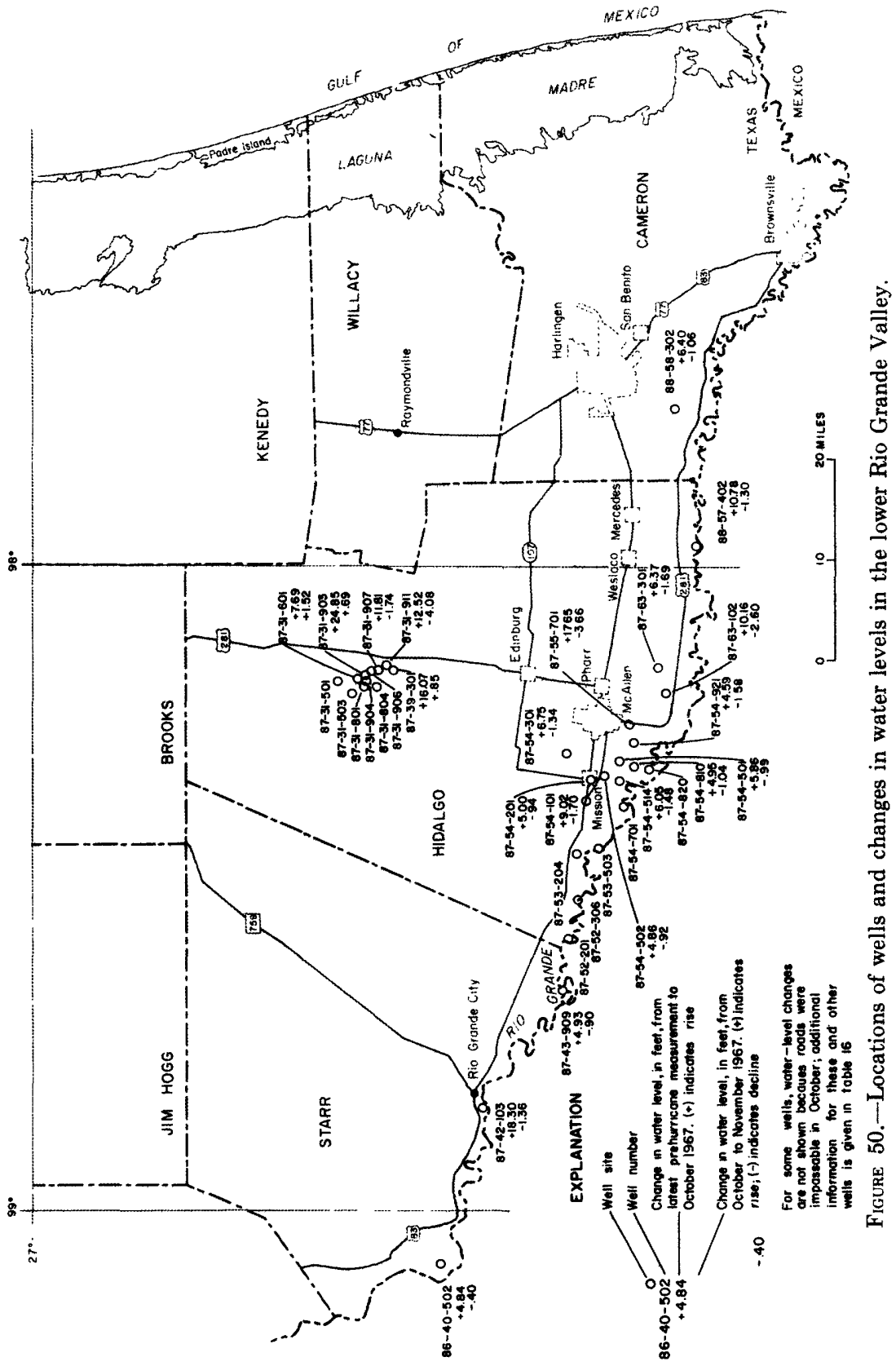


$\underset{\substack{\alpha \\ \alpha}}{\frac{\sim}{\alpha}}$

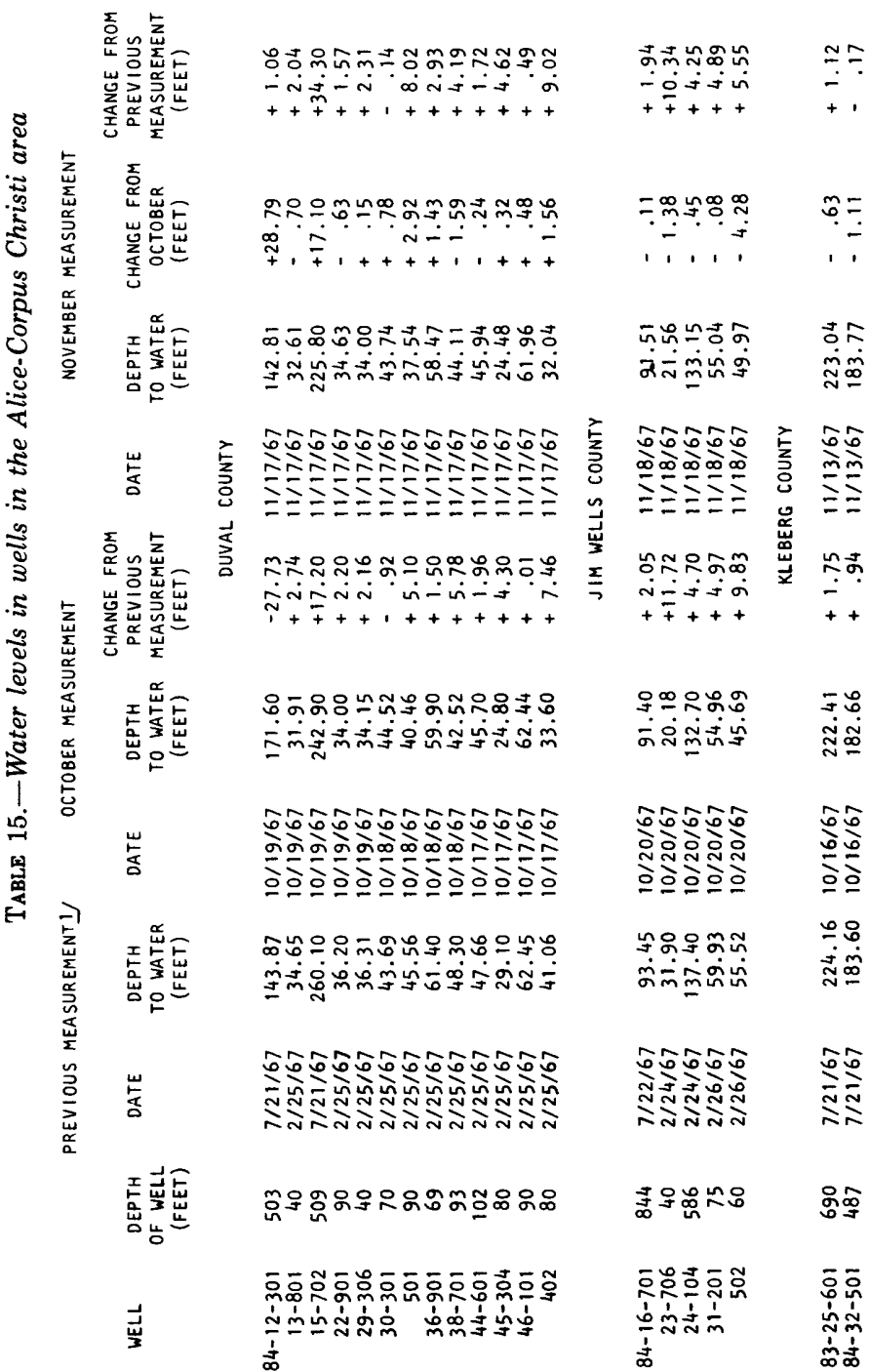




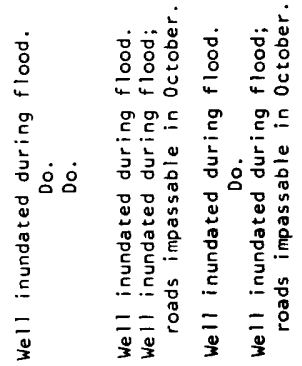

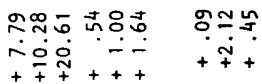

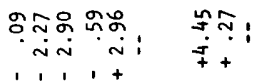

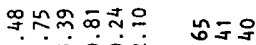

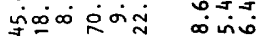

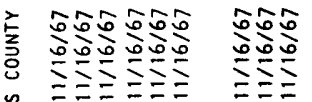

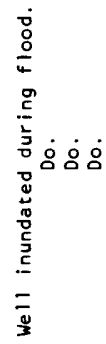

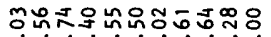

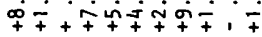

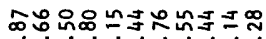
$1++\overline{+}+++i+$

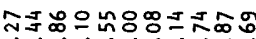
흠

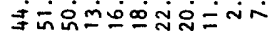

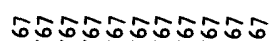

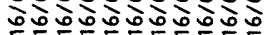
ミミミミミミミミミミミ

ㅇํำํํㅇํำํํํำำ

衣

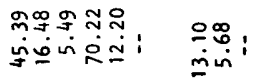

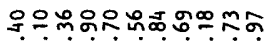

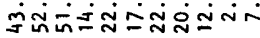

ใิำํำำ

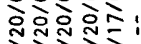

ํํำ

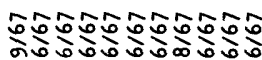

으으으으으

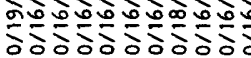

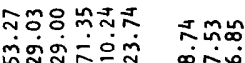

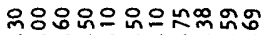

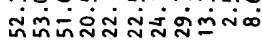

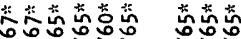

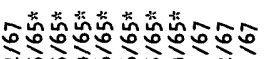

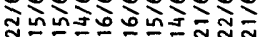

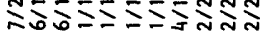

祆於

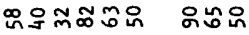

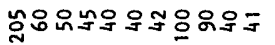

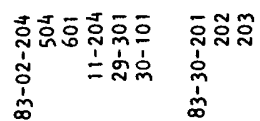

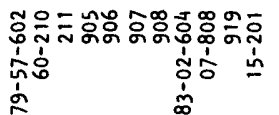




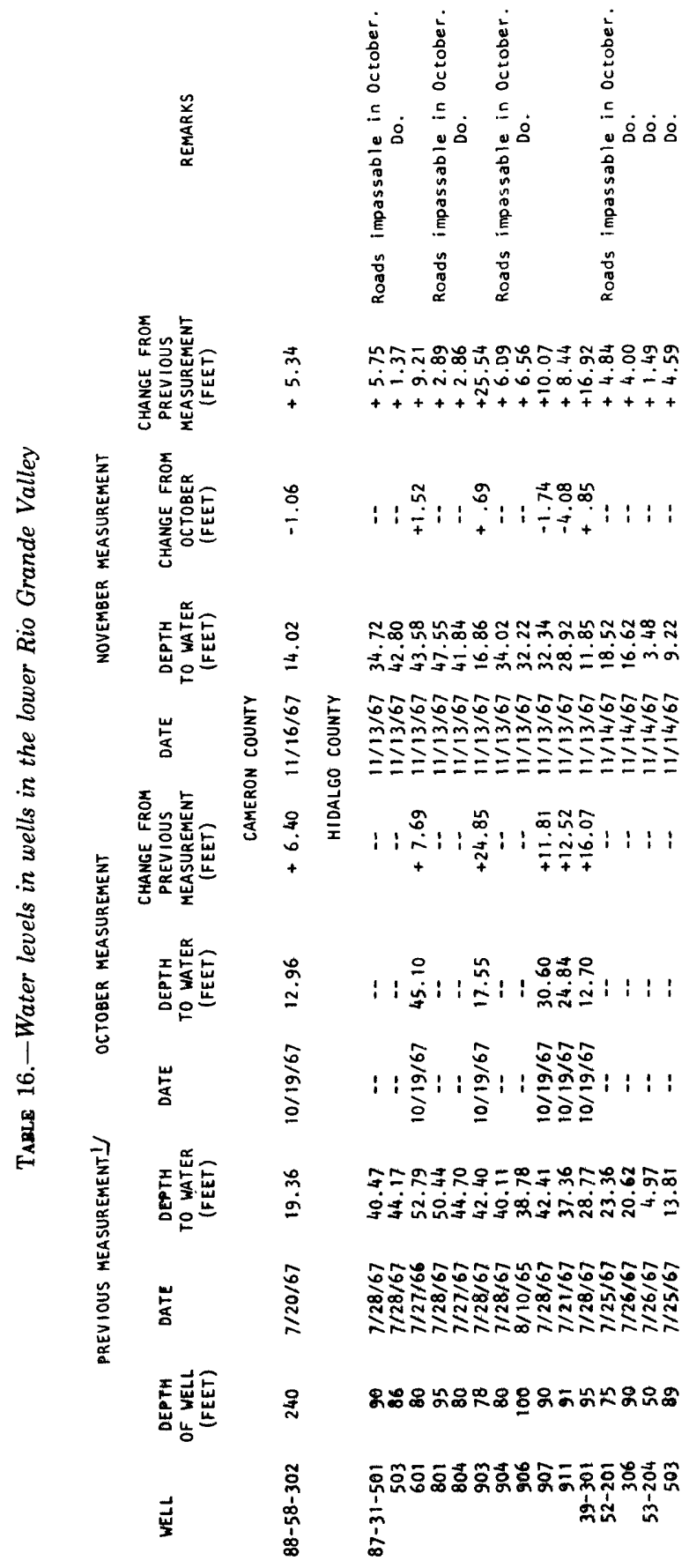




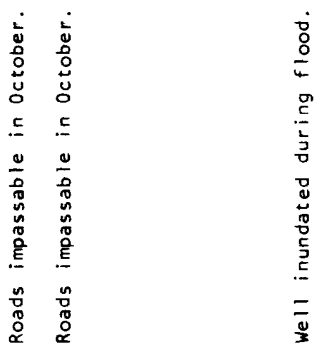

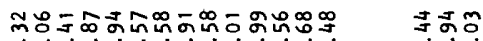
risisima mismmisa jos

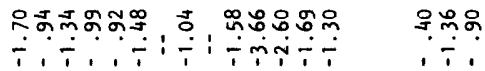

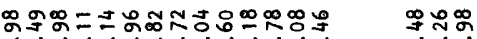

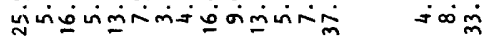

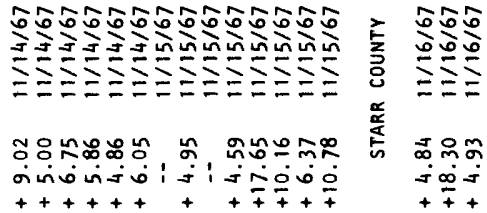

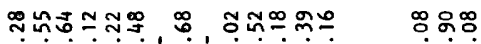

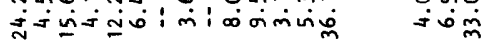

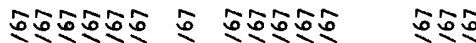

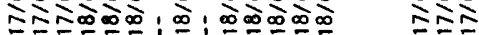

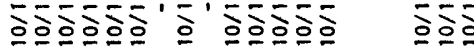

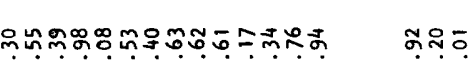

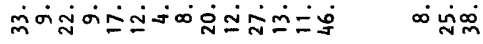

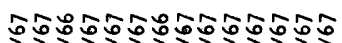

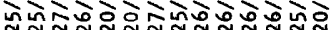

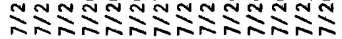

ลิอะ inin กู่

ํำ

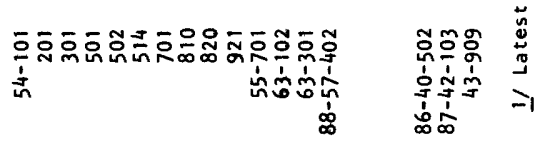



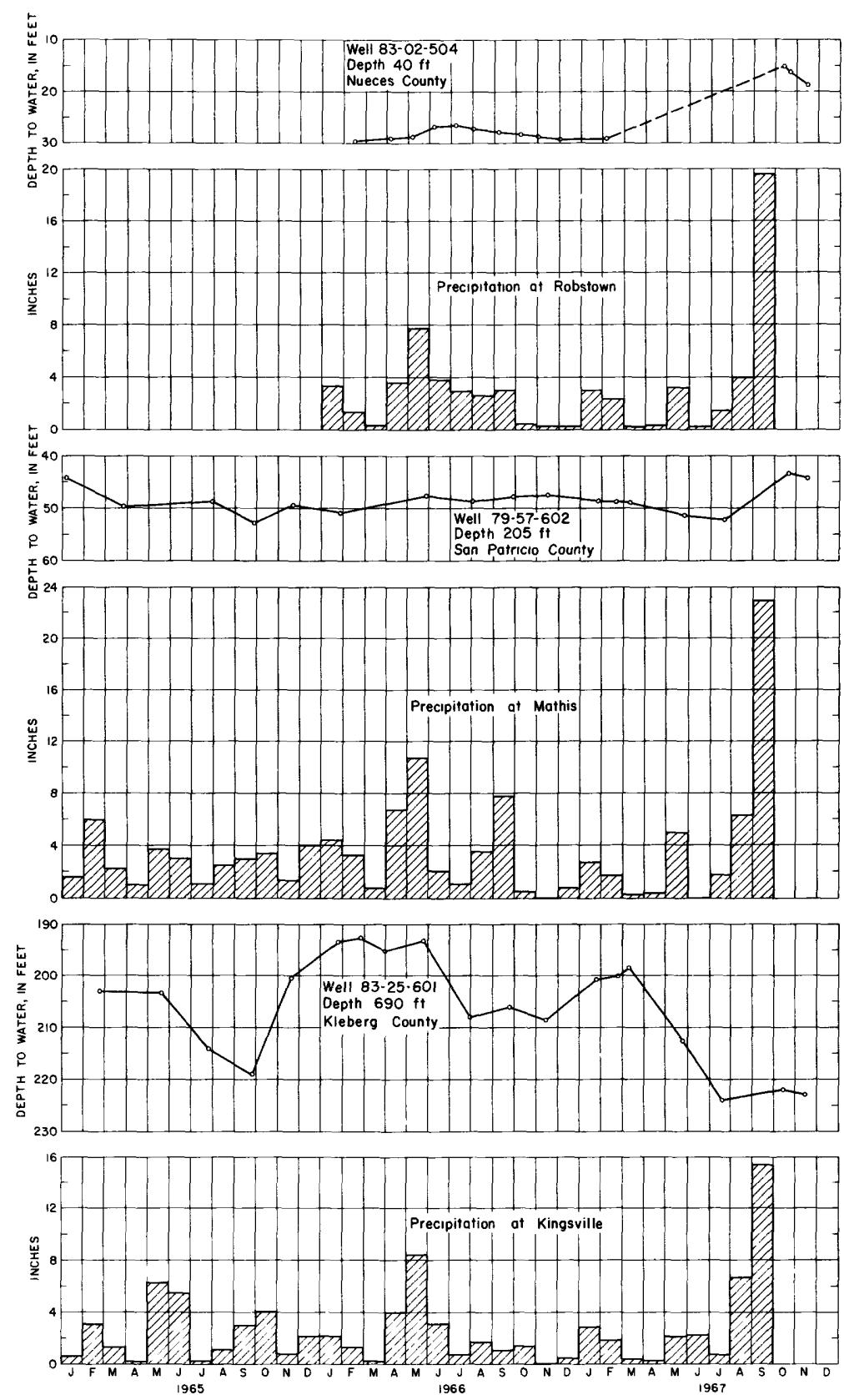

Figure 51 (above and facing page).-Hydrographs of wells and precipitation at nearby stations in the Alice-Corpus Christi area. 

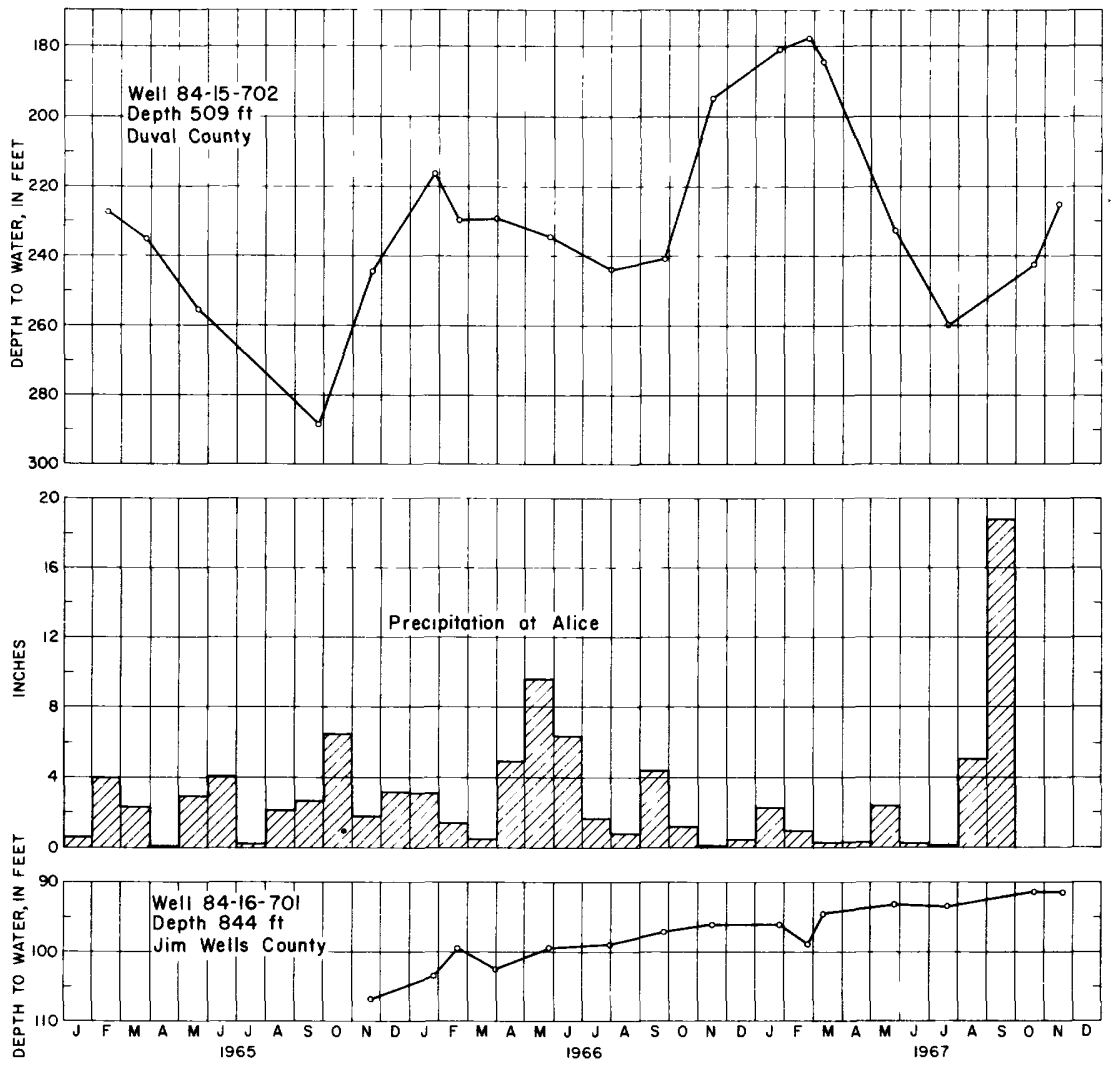

previous trends are shown for well 88-58-302 (240 ft deep) and well 87-54-502 (355 ft deep). The 1965 and 1966 water-level fluctuations in well 88-57-402 (180 ft deep) suggest that the September 1967 rise could be caused by factors other than hurricane-induced recharge.

The evidence indicates that Hurricane Beulah caused significant rises in water levels in shallow wells by percolation of rainfall and ponded waters and by the cascading of floodwaters directly into numerous inundated wells (tables 15 and 16). Water levels also rose owing to cutbacks in pumping.

Although considerable economic losses resulted from the hurricane, there were probably some benefits by partial replenishment of the ground-water reservoirs.

\section{EXPLANATION OF STATION DATA}

One of the main purposes of a flood report is presentation of stage and discharge data on streams. These data are presented in the following 

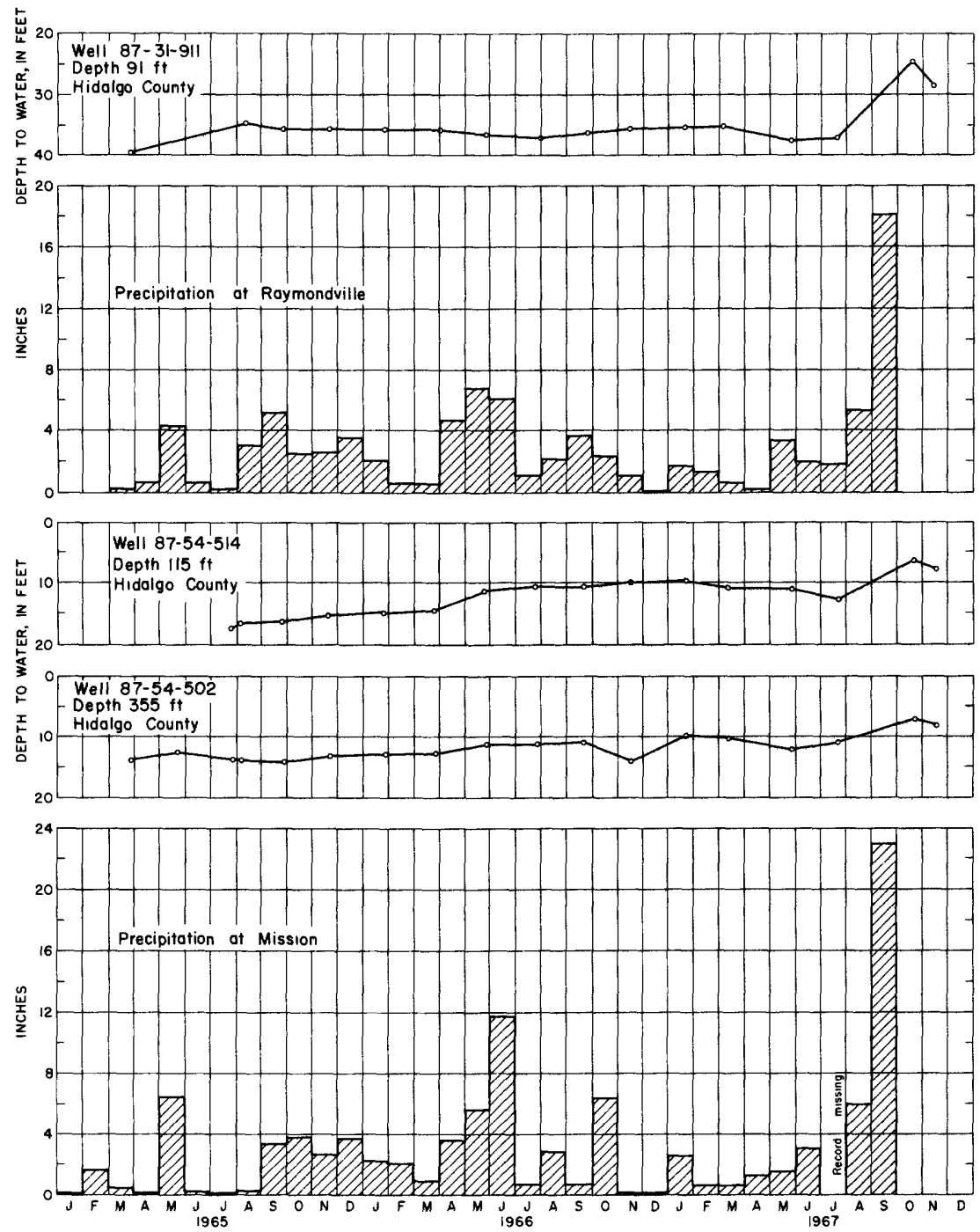

Figure 52 (above and facing page).-Hydrographs of wells and precipitation at nearby stations in the lower Rio Grande Valley.

section in sufficient detail so that hydrographs of the flood peaks may be accurately constructed. The hydrologist who needs more detailed data may contact the Texas District Office in Austin or the San Antonio Subdistrict Office of the U.S. Geological Survey.

The data consist of a description of the station or site, a table showing the daily discharge at gaging stations for September-October 1967, and tables of stage and discharge at indicated times for the gaging stations. 

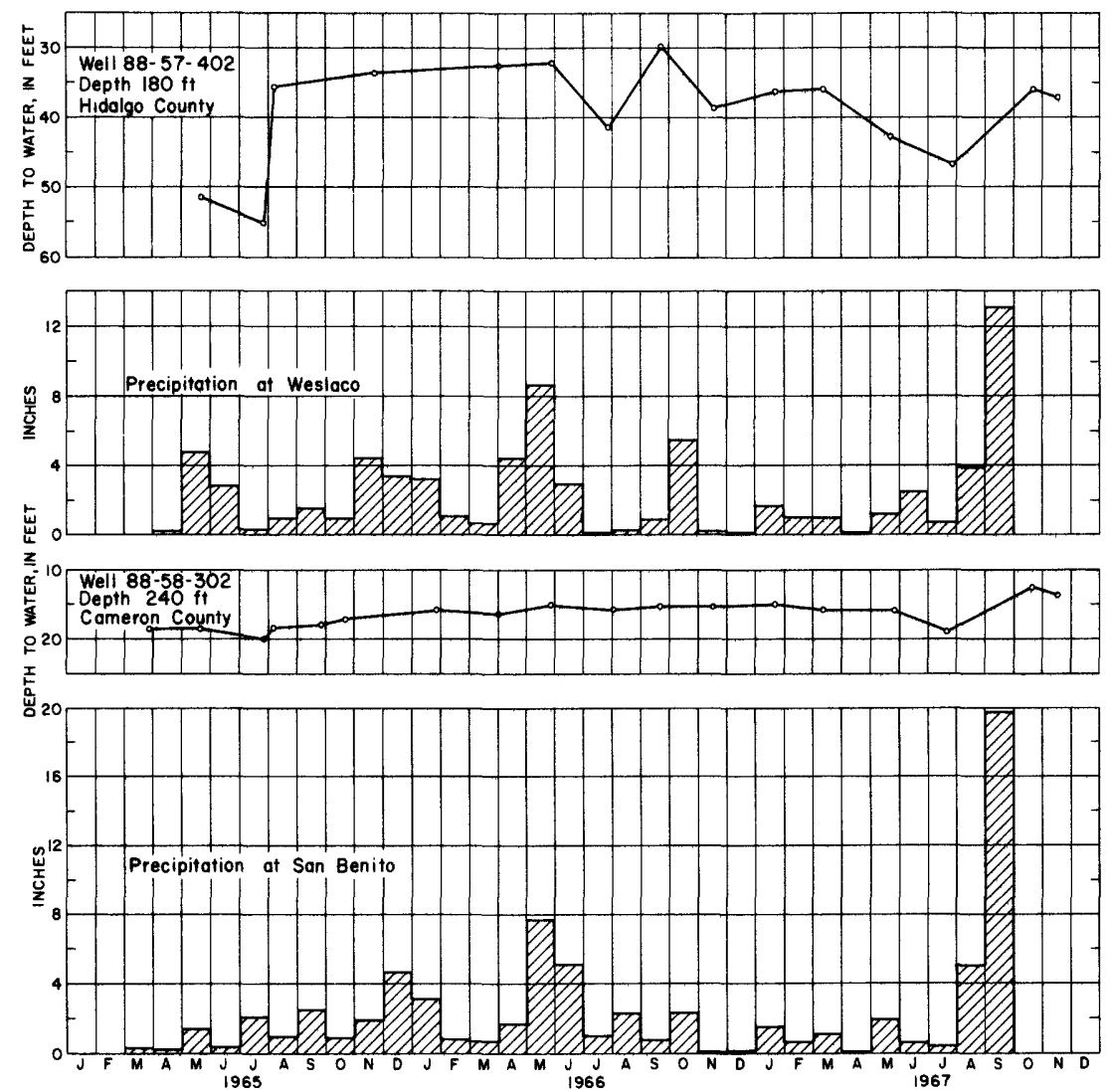

The station description gives information relative to the location of the gage, size of the drainage area above the gage, nature of the gageheight record obtained during the period covered by this report, datum of gage, definition of stage-discharge relation, maximum stage, and discharge during the September 1967 floods and previous maximum during the period of record, maximum data for floods outside the period of record, effect of regulation and diversion, and other pertinent general information.

The table of daily mean discharge gives data for the 2-month period, September-October 1967, to cover not only the period of major flooding, but a sufficient length of time to show discharges during antecedent and recession periods. The monthly figures of the table show the monthly mean discharge in cubic feet per second, the volume of monthly runoff in acre-feet, and the volume of monthly runoff in inches at the selected stations. Monthly figures for those stations downstream from a reservoir have not been adjusted for changes in contents of the reservoir.

The table of stages and discharges at indicated times gives sufficient 


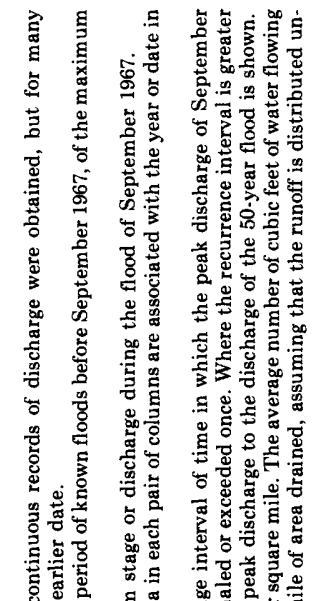

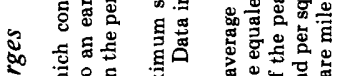
ํํำ

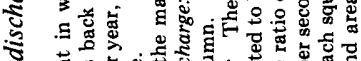

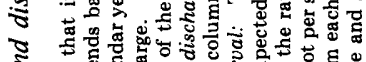

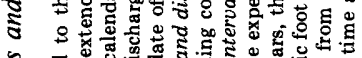
\&

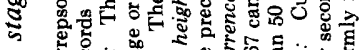

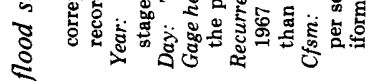

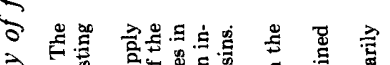

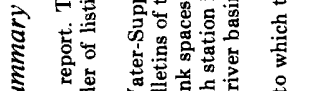

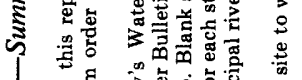

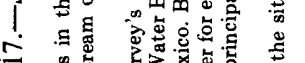

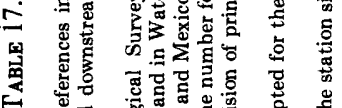

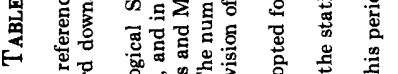
ब营

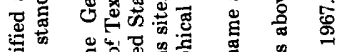

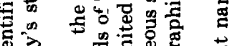

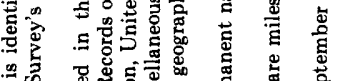

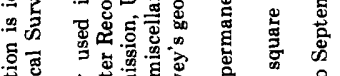
要要

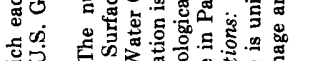

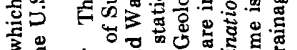

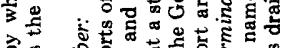

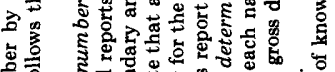
s.

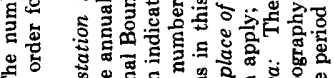
F⿻

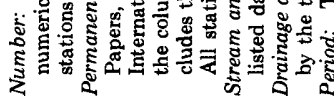

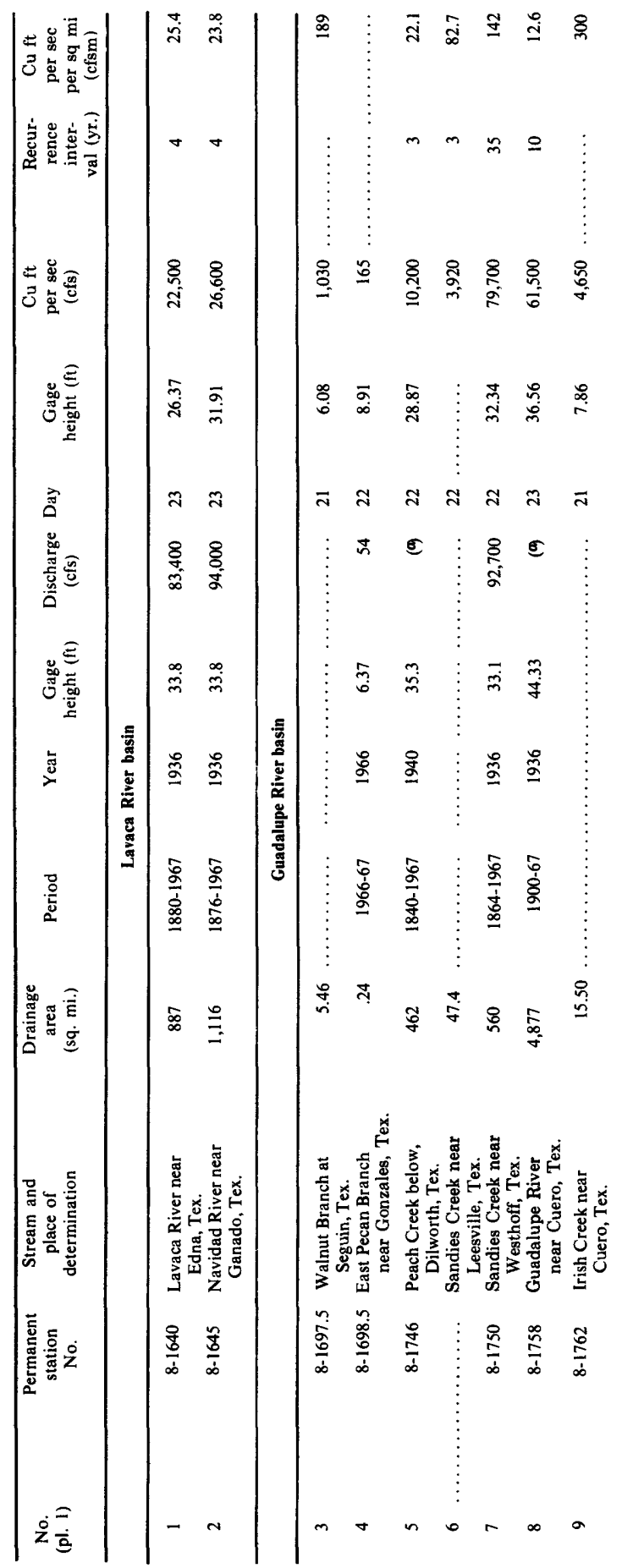


SOUTH TEXAS AND NORTHEASTERN MEXICO, SEPT.-OCT. B101

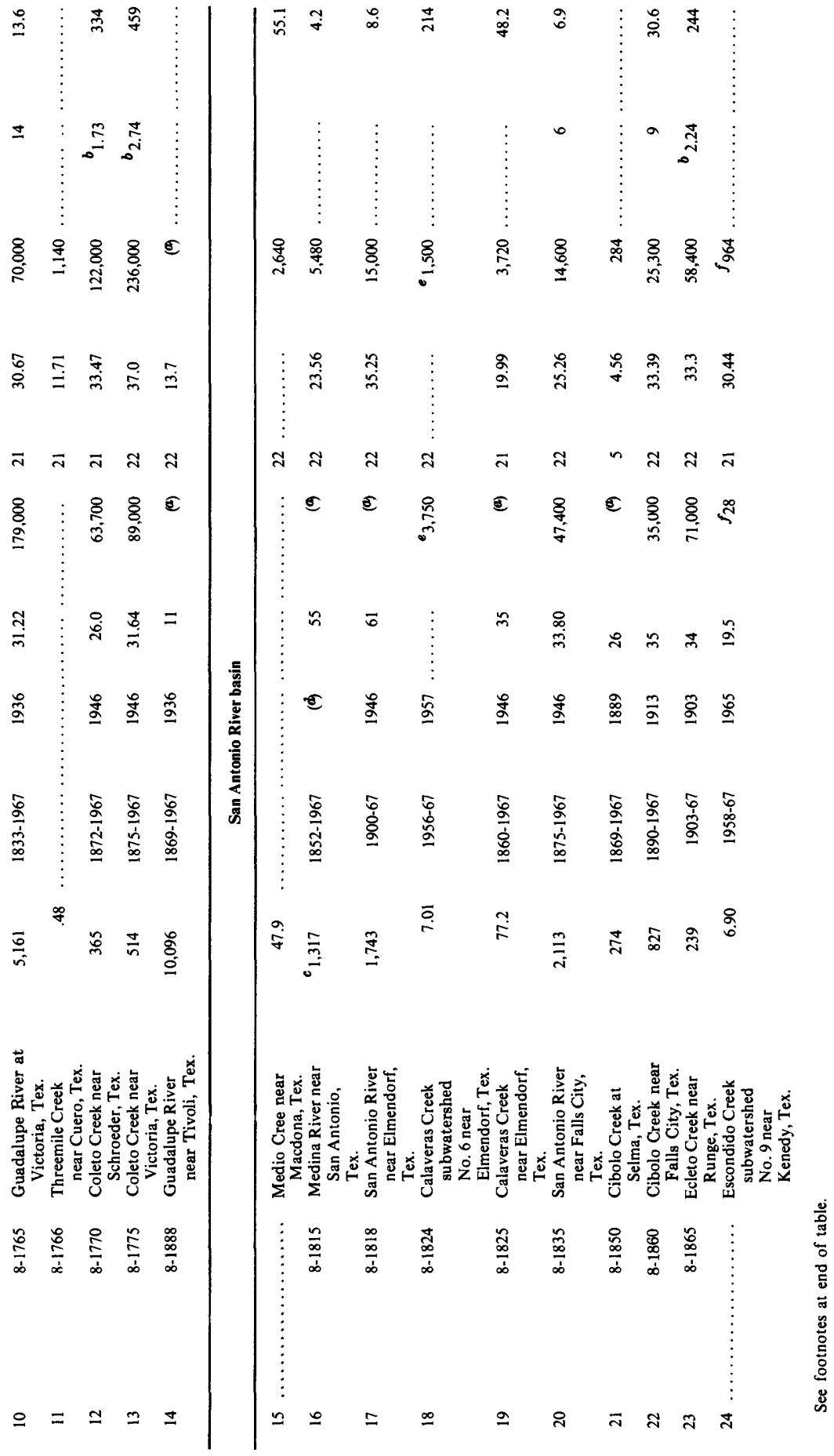




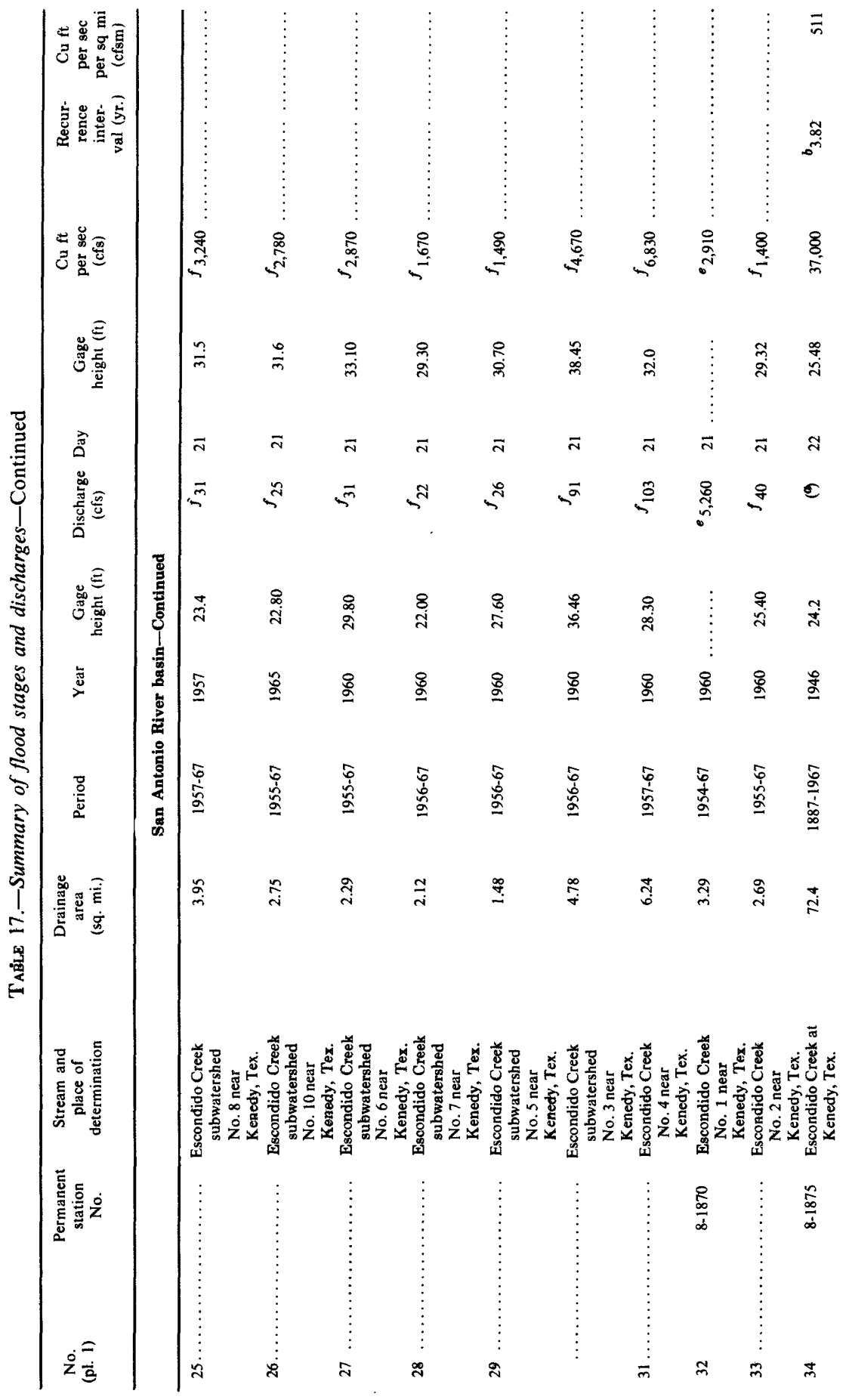


SOUTH TEXAS AND NORTHEASTERN MEXICO, SEPT.-OCT. B103

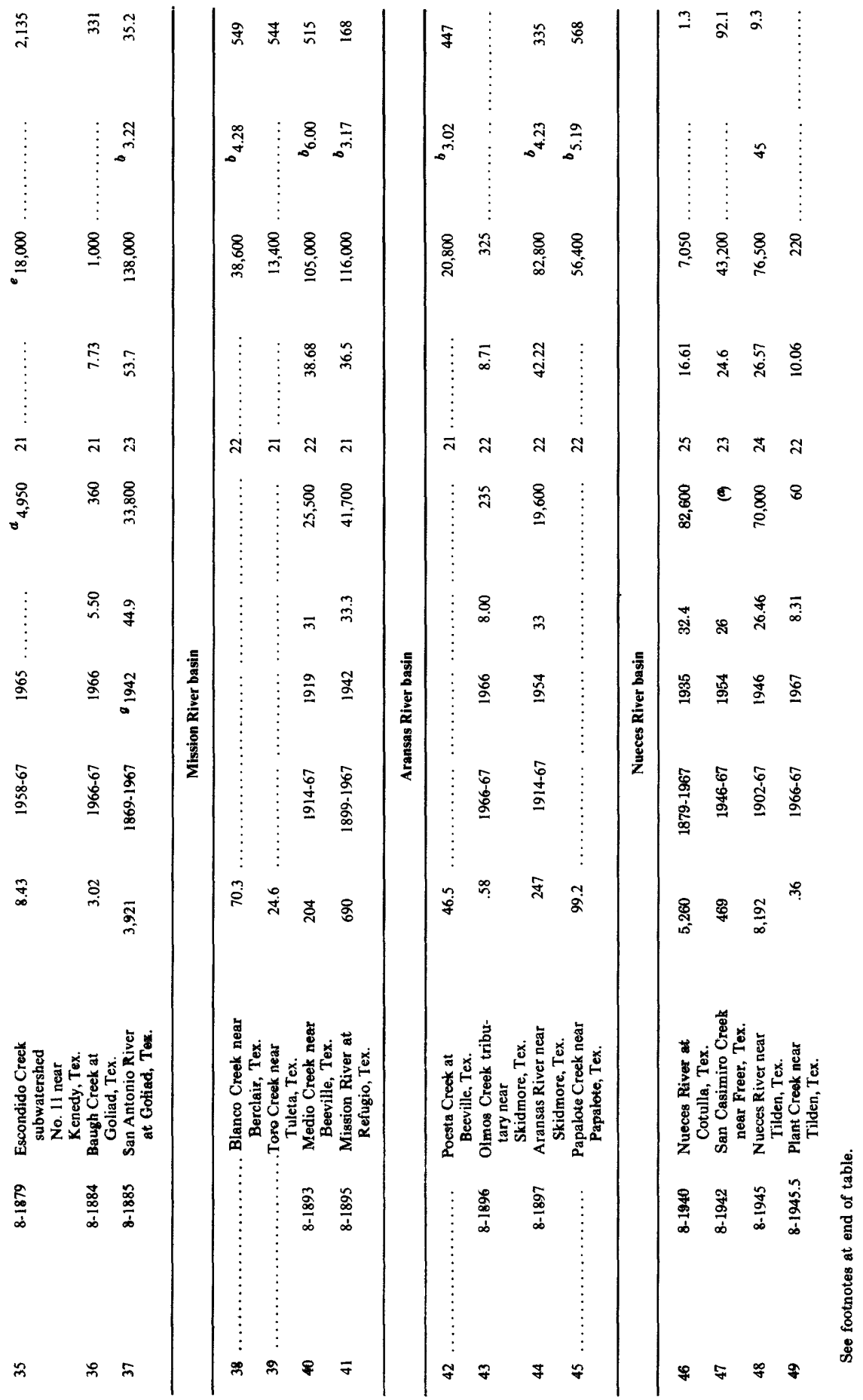




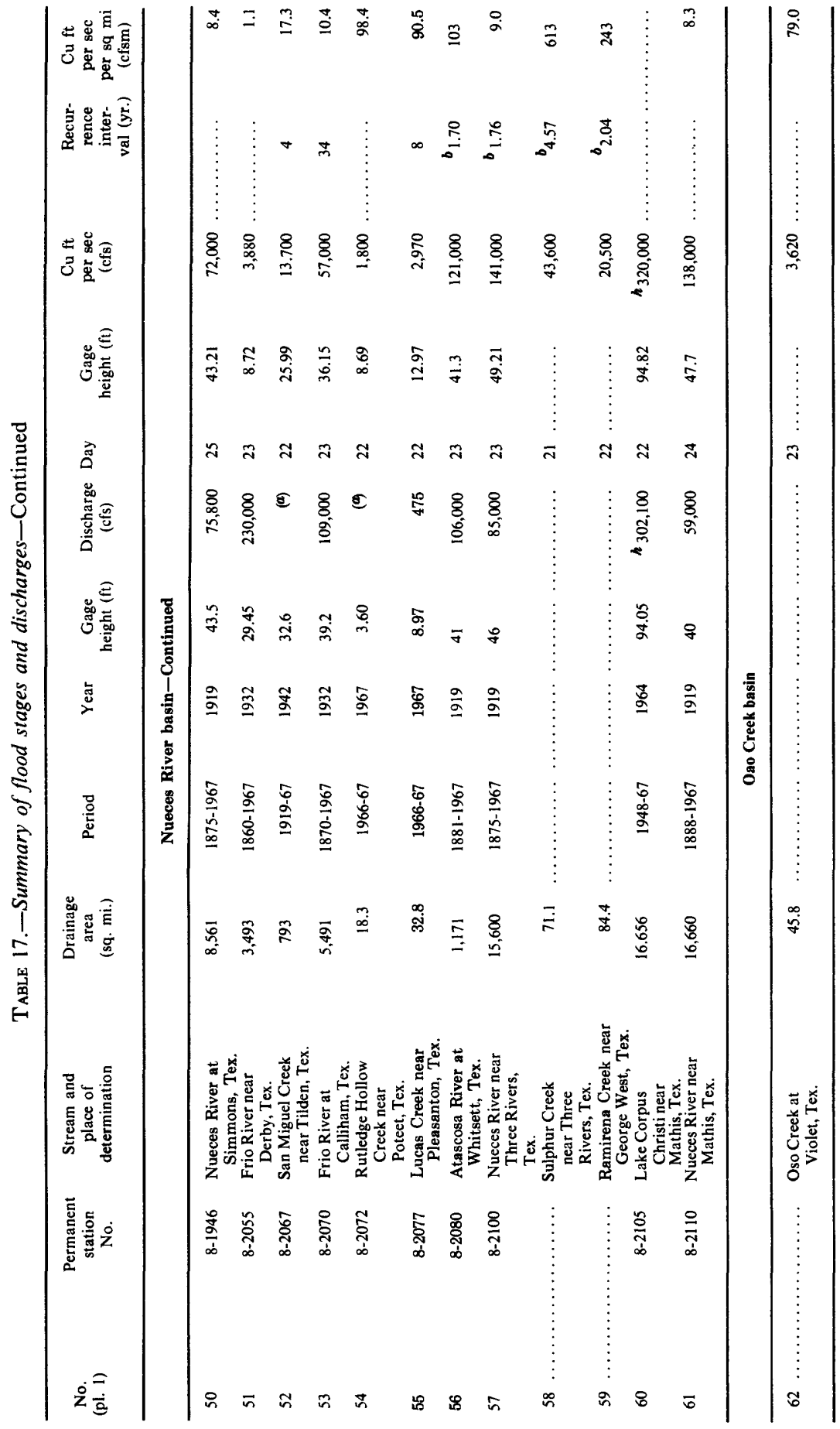


SOUTH TEXAS AND NORTHEASTERN MEXICO, SEPT.-OCT. B105

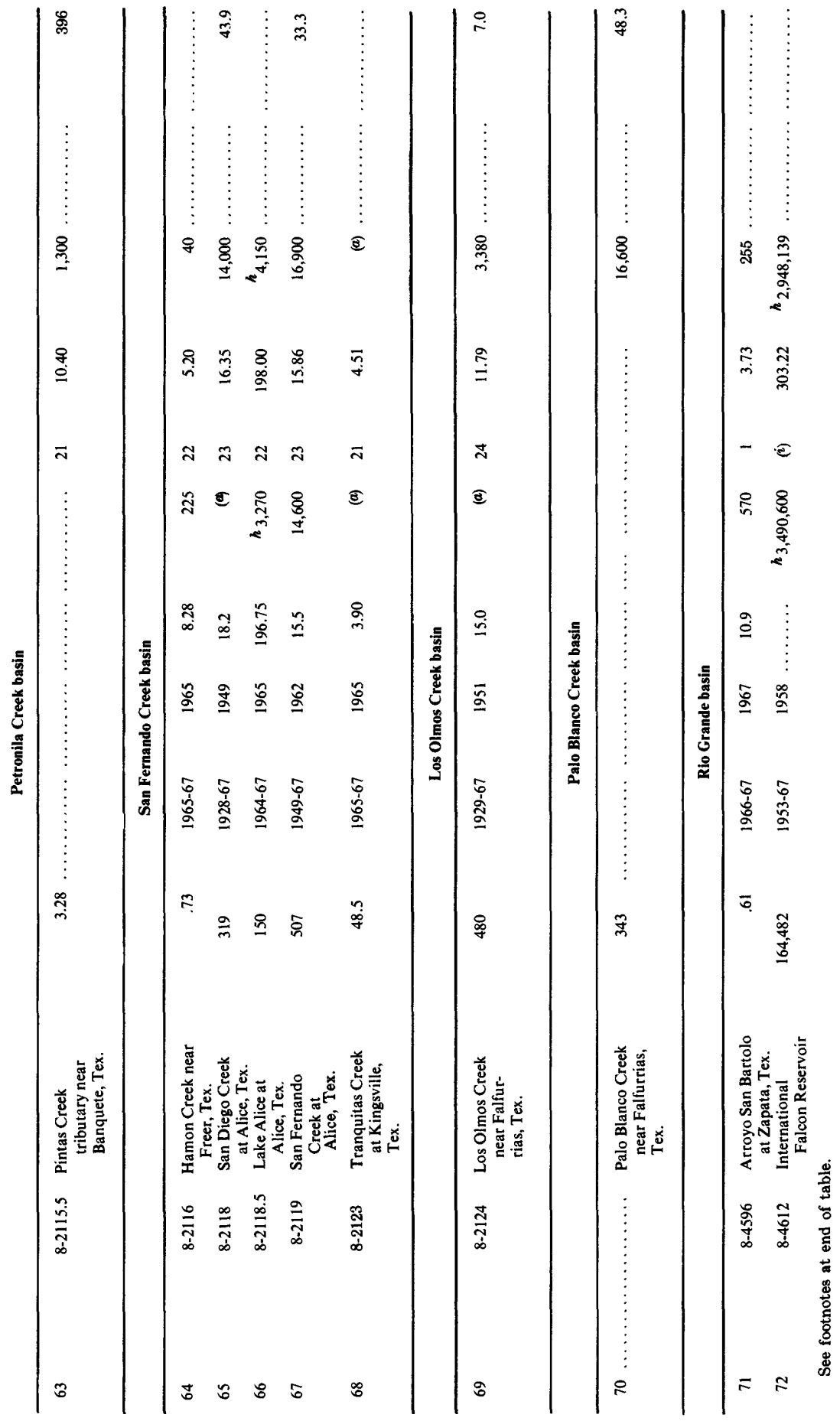




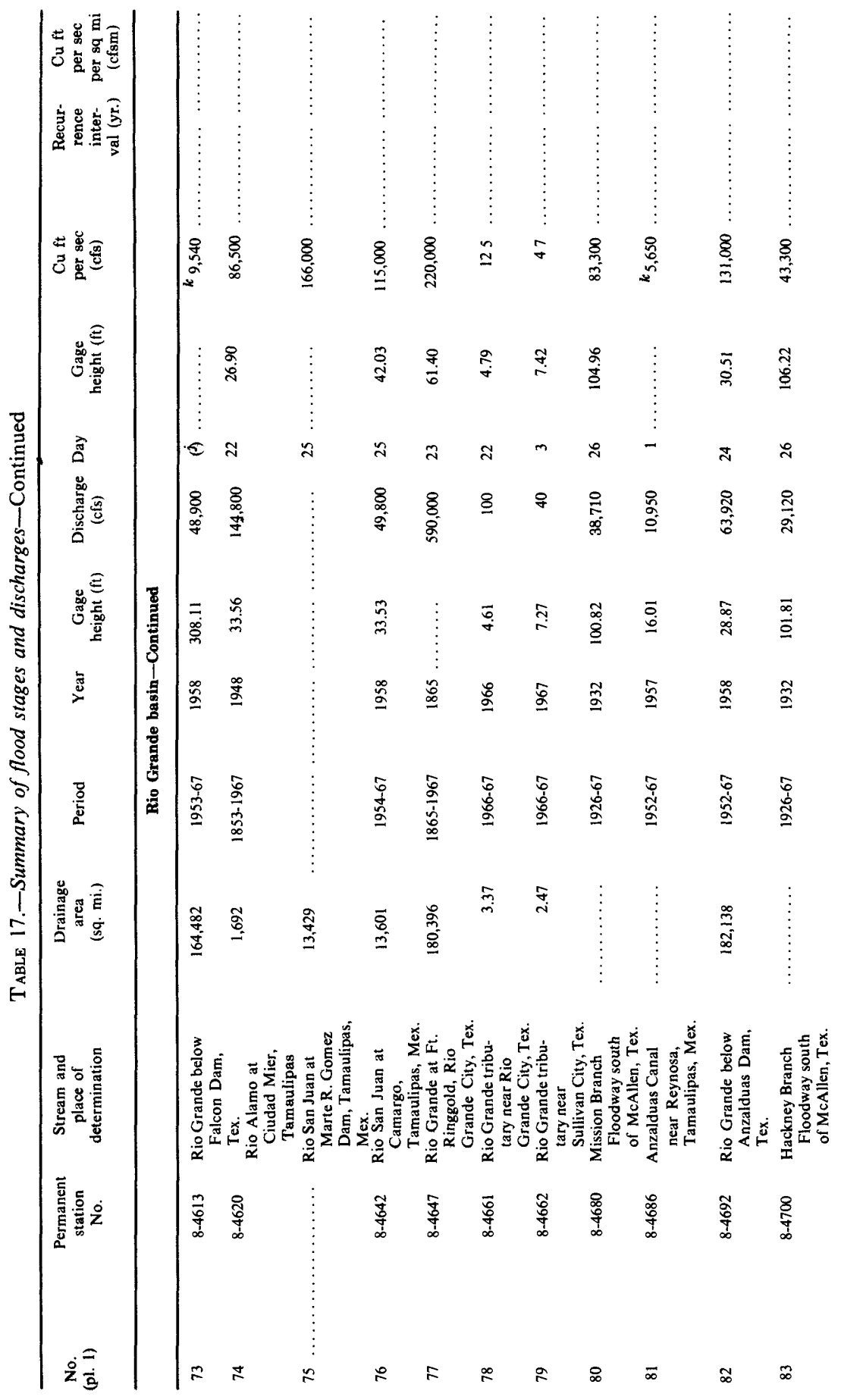




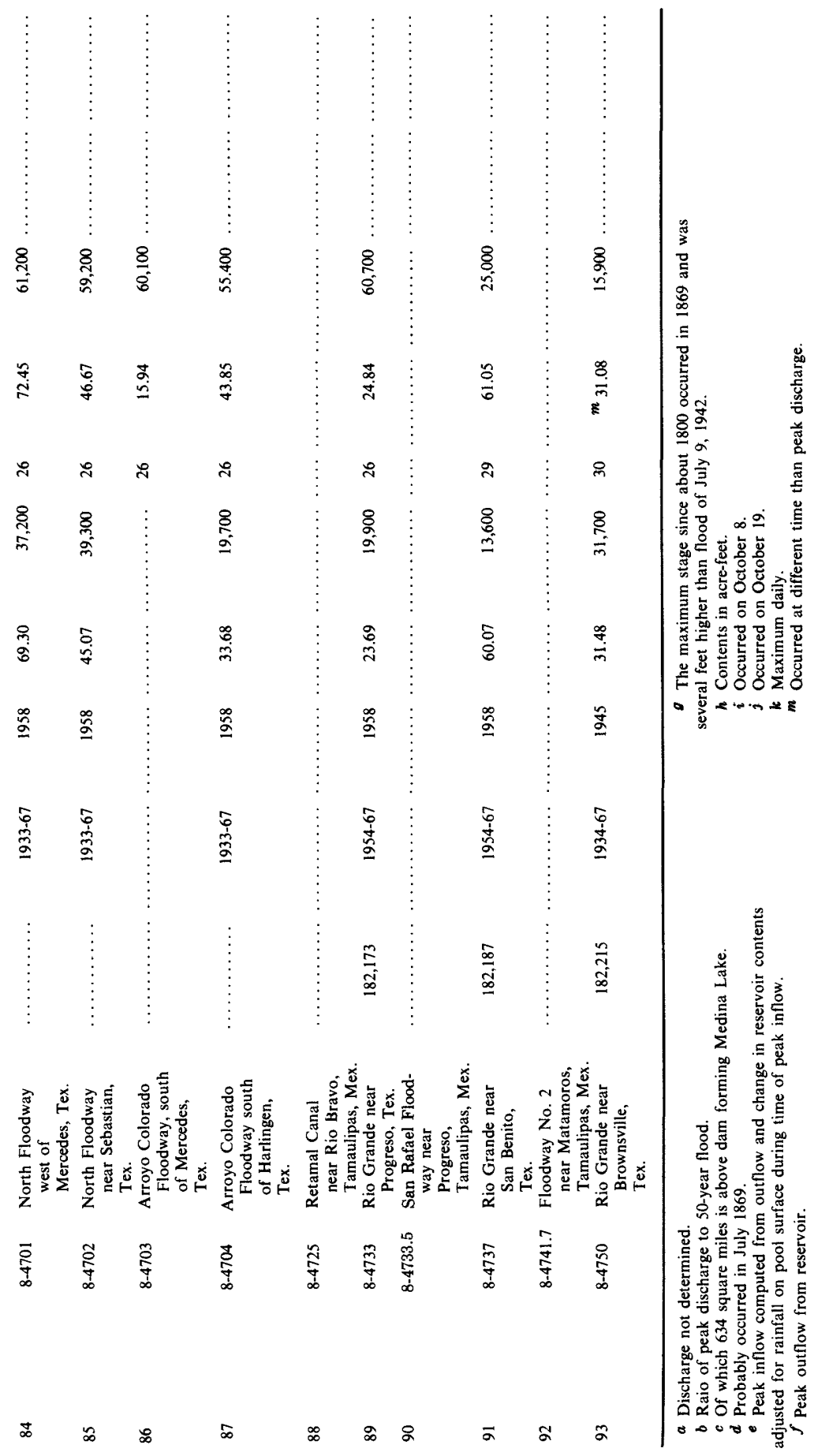


data so that hydrographs of stage and discharge can be drawn. The period of time covered is from prior to the start of the major rise to an arbitrary cutoff point on the recession and is not the same for all stations.

\section{SUMMARY OF FLOOD STAGES AND DISCHARGES}

Maximum stages and discharges at 93 gaging stations, crest-stage stations, miscellaneous sites, and reservoir stations are given in table 17. The reference numbers (site number), which correspond to those on the flood-site location map (pl. 1), are given as an aid in locating the sites at which peak discharges were determined.

The derivation of the maximum data is explained in the station description for each site. The peak discharges in table 17 are those actually determined; that is, no adjustments for storage, regulation, or diversions have been made. For reservoir stations the maximum stage and contents are given, and for some, the computed peak inflow is given.

\section{SELECTED REFERENCES}

Bice, E. G., 1968a, Hurricane Beulah lashes the lower Rio Grande Valley: Brownsville Tex., Environmental Science Services Admin., Weather Bur. Airport Sta., April, 18 p.

1968b, Climatopography of Texas: Austin, Tex., Environmental Science Services Administration, May and June, $4 \mathrm{p}$.

Commons, G. G., 1944, Flood peaks in Texas: State Board of Water Engineers, p. 4.

Gumbel, E. J., 1954, Statistical theory of extreme values and some practical applications: Natl. Bur. Standards, Appl. Math. Ser. 33, 51 p.

Hershfield, D. M., 1961, Rainfall frequency atlas of the United States: U.S. Weather Bur. Tech. Paper 40, $61 \mathrm{p}$.

Miller, J. F., 1964, Two- to 10-day precipitation for return periods of 2 to 100 years in the contiguous United States: U.S. Weather Bur. Tech. Paper 49, 29 p.

Patterson, J. L., 1965, Magnitude and frequency of floods in the United States, Part 8, Western Gulf of Mexico Basins: U.S. Geol. Survey Water-Supply Paper 1682, 506 p. 1968, Report on Hurricane Beulah, September 8-21, 1967: Galveston, Tex., U.S. Army Engineer District, Corps of Engineers, September 1968, 26 p., 5 tables, 76 plates, 21 exhibits. 


\section{INDEIX}

[Italic page numbers indicate major references]

A Page

Acknowledgments

Alice, Tex

Alice-Corpus Christi area, water-

level fluctuations

Anzalduas Dam

Aransas River near Skidmore, Tex

Arroyo Colorado

Atascosa River, Tex

flooding at Three Rivers, Tex

Atascosa River at Whitsett, Tex

\section{B}

Baffin Bay, Tex

Baluarte Creek, Tex

Barahona Peninsula, Dominican Republic ......

Baugh Creek at Goliad, Tex

Bays, results of freshwater inflow

Beeville, Tex

Blanco Creek near Berclair, Tex .............

Brownsville, Tex

C

Calallen, Tex

Calaveras Creek, Tex

Calaveras Creek watershed

Campbellton, Tex

Carmargo, Tamaulipas, Mexico

Chiltipin Creek, Tex.

Cibolo Creek, Tex

$28,30.46$

Cibolo Creek at Selma, Tex

Cibolo Creek near Falls City,

$$
\text { Tex }
$$

Cibolo Creek watershed, Tex

Ciudad Mier, Tamaulipas, Mexic

Coastal area between the Nueces

$$
\text { River and the Rio Giande........ }
$$

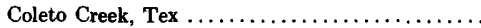

Coleto Creek near Schroeder,

$$
\text { Tex } \ldots \ldots \ldots \ldots
$$

Coleto Creek near Victoria, Tex

Coleto Creek watershed, Tex .............. 25

Commons curve, magnitude of floods . . . . .52, 53, 55

Corpus Christi, Tex ............. 5, 7, 43, 45

Corpus Christi-Alice area, waterlevel fluctuations.

Corpus Christi Bay.

coastal area

freshwater inflow

Cotulla, Tex.

Cozumel Island
C'uero, Tex

Page

23,27

\section{D}

Damage survey $\ldots \ldots \ldots \ldots \ldots \ldots \ldots \ldots \ldots \ldots 664,66$

Derby, Tex $\ldots \ldots \ldots \ldots \ldots \ldots \ldots \ldots \ldots \ldots \ldots, 40$

Dilworth, Tex ..................... 23

Dissolved solids, Guadelupe-San

Antonio Bay ............. 87

Iissolved solids, Mathis-Calallen reach,

Nueces River ................ 86

Iissolved solids, Nueces-Corpus Christi

Bay $\ldots \ldots \ldots \ldots \ldots \ldots \ldots \ldots \ldots, 84$

\section{E}

Ecleto Creek, Tex ................. 28, 34

Ecleto Creek near Runge . . . . . . . . . . . 31

Ecleto Creek watershed, Tex ............ 31

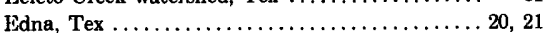

Ilmendorf, Tex $\ldots \ldots \ldots \ldots \ldots \ldots \ldots \ldots \ldots \ldots \ldots$.

Encino, Tex .................... 47

Encino crossroads gage, King Ranch ......... 6

Encino headquarters, King Ranch ........... 5

Escondido Creek, Tex $\ldots \ldots \ldots \ldots \ldots \ldots \ldots \ldots 28,34,45$

Escondido Creek at Kenedy, Tex ............ 34

Escondido Creek watershed $\ldots \ldots \ldots \ldots \ldots \ldots \ldots \ldots$ 2, 34

flood-volume estimates $\ldots \ldots \ldots \ldots \ldots \ldots \ldots .60$

Estuaries, results of freshwater inflow . . . . . . 67

\section{$\mathbf{F}$}

Falcon Dam, Rio Grande ............. 50

Falfurrias, Tex .................. 45, 46

Falls City, Tex ....................... 31

Flood damage, summary $\ldots \ldots \ldots \ldots \ldots \ldots \ldots, 64$

Flood discharges, summary ............. 108

Flood stages, summary $\ldots \ldots \ldots \ldots \ldots \ldots \ldots \ldots, 108$

jlood volume $\ldots \ldots \ldots \ldots \ldots \ldots \ldots \ldots \ldots \ldots \ldots, 60$

Floodway No. 2, Mexico, downstream from

Anzalduas Dam ............. $\quad 50$

Trequency of the floods $\ldots \ldots \ldots \ldots \ldots \ldots \ldots \ldots, 50$

Fireshwater inflow, results to bays

and estuaries $\ldots \ldots \ldots \ldots \ldots \ldots \ldots, 67$

Frio River, Tex . . . . . . . . . . . . . . 40

flood-frequency relations $\ldots \ldots \ldots \ldots \ldots \ldots \ldots, 51$

flooding at Three Rivers, Tex ......... 42

Frio River at Calliham, Tex ............. 41

Frio River at Simmons, Tex . . . . . . . . . . 41

Frio River watershed, Tex $\ldots \ldots \ldots \ldots \ldots \ldots \ldots 40,41$

G

Gamble Gully, Tex $\ldots \ldots \ldots \ldots \ldots \ldots \ldots \ldots \ldots \ldots$

Ganado, Tex ........................ 21

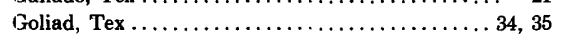


Guadalupe River at Cuero, Tex

Guadalupe River at Victoria, Tex

Guadalupe River near Victoria, Tex

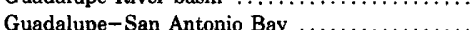

Gulf of Mexico . ................ 4, 45, 46, 47

\section{H}

Hackney Inlet

Hearne, Tex $\ldots \ldots \ldots, \ldots \ldots \ldots \ldots \ldots \ldots \ldots$

Houston, Tex

Hurricane Beulah deaths attributed ........................ description of storm

total damages caused

\section{J, K}

Jarvis-Myers curve, magnitude of floods

Kenedy, Tex

Kerrville, Tex

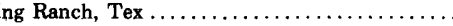

Norias Division

dairy dam

Kingsville, Tex., dam failure on Santa

Gertrudis Creek

\section{L}

La Gloria, Tex

La Parra Creek, Tex

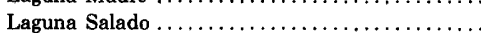

Lake Alice, Tex .......................

Lake Corpus Christi, Tex ............... 42, 43

Lavaca Bay, Tex ........................

Lavaca River, Tex

Lavaca River basin

Leesville, Tex ....

Los Olmos Creek, Tex

dischargek, Tex $\ldots \ldots \ldots \ldots \ldots \ldots \ldots \ldots$

Los Olmos Creek at Baffin Bay, Tex

Los Olmos Creek north of Falfurrias, Tex

Lower Rio Grande Valley, water-level measurements

\section{M}

Macdona, Tex

Magnitude of the floods

Marte Gomez Dam, Rio San Juan,

$$
\text { Mexico }
$$

Marte Gomez Reservoi

Matagorda Bay, Tex

Mathis, Tex

Medina, Tex

Medina River, Tex $\ldots \ldots \ldots \ldots \ldots \ldots \ldots \ldots \ldots \ldots 28,29$

Medina River near Macdona, Tex .........., 30

Medina River watershed ................... 29

Medio Creek, Tex ....................2, 29, 35

Medio Creek at Pettus, Tex

Mission River upstream from Refugio,

Tex $\ldots \ldots \ldots \ldots \ldots \ldots \ldots \ldots \ldots, \quad 35$

Medio Creek near Beeville, Tex ............. 36

Mercedes, Tex $\ldots \ldots \ldots \ldots \ldots \ldots \ldots \ldots \ldots \ldots .49$

Mission, Tex ......................... 48

Mission Inlet $\ldots \ldots \ldots \ldots \ldots \ldots \ldots \ldots \ldots, 48$

Mission River at Refugio, Tex ............. 36

\section{$\mathrm{N}$}

Navidad River near Ganado, Tex ............ 21

Norias headquarters, King Ranch ........... 47

Nueces Bay, Tex .................... 4, 43

Nueces-Corpus Christi Bay ............... 84

Nueces River, flood-frequency relations ......... 50

flooding at Three Rivers, Tex ............ 42

Nueces River at Cotulla, Tex.............. 41

Nueces River basin $\ldots \ldots \ldots \ldots \ldots \ldots \ldots \ldots \ldots \ldots, 2,39$

Nueces River below Cotulla, Tex ............ 41

Nueces River near Mathis, Tex ............ 39, 43

Nueces River near Three Rivers,

$$
\text { Tex } \ldots \ldots \ldots \ldots \ldots \ldots \ldots \ldots, 40,43
$$

Nueces River near Tilden, Tex ............. 41

\section{o}

Oakville, Tex $\ldots \ldots \ldots \ldots \ldots \ldots \ldots \ldots \ldots, \quad 42$

Olmos Creek tributary near Skidmore

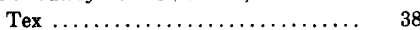

Opossum Creek, Tex ................... 41

Oso Creek near Violet, Tex .............. 45

\section{$\mathbf{P}$}

Palo Blanco Creek west of Fal-

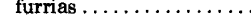

Papalote, Tex ......................... 38

Papalote Creek near Papalote, Tex .......... 38

Peach Creek, Tex ....................... 23

Petronila Creek, Tex .................... $\quad 45$

Pettus, Tex ......................... 35, 37

Plant Creek near Tilden, Tex $\ldots \ldots \ldots \ldots \ldots \ldots, 41$

Poesta Creek, Tex ...................... 38, 39

Port Brownsville $\ldots \ldots \ldots \ldots \ldots \ldots \ldots \ldots \ldots \ldots, 5$

Poteet, Tex ......................... 40

Rainfall measurements ...................

Ramirena Creek south of George West,

Refugio, Tex $\ldots \ldots \ldots \ldots \ldots \ldots \ldots \ldots \ldots \ldots, 36$

Retamal Heading floodway, Mexico ........... 50

Rio Alamo, Mexico ........................

Rio Alamo watershed, Mexico ...........5, 15, 47

Rio Grande ............................ 46

Falcon Dam ...................... 50

Mexican side, principal floodways $\ldots \ldots \ldots \ldots 49,50$

Rio Grande at Fort Ringgold, Tex ........... 48

Rio Grande basin ...................... 47

below Falcon Dam ................... 2

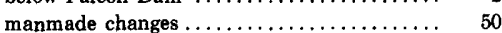

Rio Grande below Falcon Dam $\ldots \ldots \ldots \ldots \ldots, 47,48$
Rio Grande near Rio Grande City, Tex $\ldots \ldots \ldots \ldots$ 
Rio Grande Valley, lower, flood

$$
\text { damage. . . . }
$$

Southern Pacific Railroad ................

50 Station data, explanation $\ldots \ldots \ldots \ldots \ldots \ldots \ldots \ldots 97$

Rio San Juan, Mexico

2 Sulphur Creek near Oakville, Tex .......... 42

Marte Gomez Dam

50

Rio San Juan watershed, Mexico . . . . . . . . 15, 48

Runge, Tex .

Rutledge Hollow Creek near Poteet,

Tex

$\mathbf{S}$

San Antonio, Tex

San Antonio Bay

San Antonio River, Tex

San Antonio River at Goliad,

Tex

San Antonio River at Medina, Tex

San Antonio River basin

Texas Water Development Board, "Effects

$\mathbf{T}$

Thrall, of the Hurricane on Water Levels in Wells"

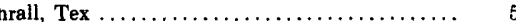
flood $w$ olume estimates $\ldots \ldots \ldots \ldots \ldots \ldots \ldots, 60$

7, 29 Three Rivers, Tex ..............40, 41, 42

4 Tiburon Peninsula, Haiti .............. 4

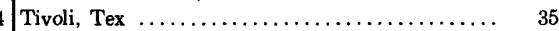

Toro Creek near Tuleta, Tex ............ 36

34, 35 Tranquitas Creek, Tex ............... 45

28 Tranquitas Reservoir, Tex............. 45

San Casimiro Creek near Freer

Tex

2,28

Tuleta, Tex ..................... 36

San Fernando Creek at Alice Tex

San Fernando Creek north of Kingsville,

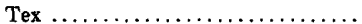

San Marcos River, Tex

San Miguel Creek near Tilden, Tex ..........

San Rafael floodway, Mexico

Sandies Creek, Tex .

Sandies Creek near Leesville

Sandies Creek near Westhoff

Sandies Creek watershed, Tex

Santa Gertrudis Creek, Tex

St. Lucia, Dominican Republic

Schroeder, Tex.

U, V

Una de Gato.

Victoria, Tex

23

40

50

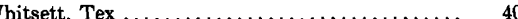

Wind damages $\ldots \ldots \ldots \ldots \ldots \ldots \ldots \ldots \ldots \ldots, 64$

Selected references $\ldots \ldots \ldots \ldots \ldots \ldots \ldots \ldots \ldots \ldots, 108$

Selma, Tex ......................... 31

Sierra Madre, near Monterrey, Mexico .........

Sinton, Tex . . . . . . . . . .

Skidmore, Tex
5 Yorktown, Tex $\ldots \ldots \ldots \ldots \ldots \ldots \ldots \ldots \ldots \ldots \ldots$

37

Yucatan Peninsula $\ldots \ldots \ldots \ldots \ldots \ldots \ldots \ldots \ldots$

' U.S. GOVERNMENT PRINTING OFFICE: $19740-585-467 / 24$ 


